\title{
PASSIVE LOCALIZATION OF UNDERWATER ACOUSTIC BEACONS
}

\author{
by \\ Dennis Michael Wojcik \\ B.S. Electrical Engineering \\ Marquette University (1986) \\ Submitted to the \\ Massachusetts Institute of Technology/ \\ Woods Hole Oceanographic Institution \\ Joint Program in Oceanography and \\ Applied Ocean Science and Engineering in \\ Partial Fulfillment of the Requirements of the Degree o \\ Master of Science in Oceanographic Engineering \\ at the \\ Massachusetts Institute of Technology and the \\ Woods Hole Oceanographic Institution

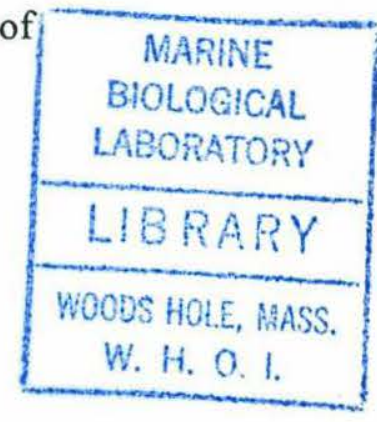 \\ September 1993 \\ (C) Dennis Michael Wojcik 1993 \\ The author hereby grants to the Massachusetts Institute of Technology, \\ the Woods Hole Oceanographic Institution, and the U.S. Government \\ permission to reproduce and distribute copies of this thesis in whole or in part.
}

Signature of Author De

Joint Program in Applied Ocean Science and Cceanographic Engineering

Massachusetts Institute of Technology/Woods Hole Oceanographic Institution

Certified by

Dr. Albert M. Bradley

Senior Engineer, Woods Hole Oceanographic Institute

Accepted by

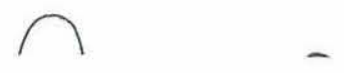

Prof. Arthur N. Baggeroer

Chairman, Joint Committee for

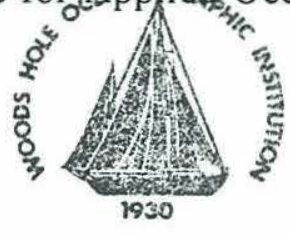




\title{
PASSIVE LOCALIZATION OF UNDERWATER ACOUSTIC BEACONS
}

\author{
by \\ Dennis Michael Wojcik \\ B.S. Electrical Engineering \\ Marquette University (1986)
}

Submitted to the Massachusetts Institute of Technology/

Woods Hole Oceanographic Institution Joint Program in

Oceanography and Applied Ocean Science and Engineering in

Partial Fulfillment of the Requirements of the Degree of

Master of Science in Oceanographic Engineering

This thesis examines the use of a single, omnidirectional hydrophone as a receiving sensor to passively localize an acoustic beacon. The localization problem is presented as a constrained, nonlinear parameter estimation problem, and Lagrange multipliers are introduced to solve for the maximum likelihood estimate of the acoustic beacon's position. An iterative algorithm is developed using range difference measurements to solve for the maximum likelihood estimate of a stationary acoustic beacon's position. This algorithm is then extended to include linear, constant velocity motion of the acoustic beacon. Finally, design specifications for a receiver to implement the maximum likelihood estimation algorithms are developed.

To test the maximum likelihood estimate algorithms, Monte Carlo simulations are conducted. Results from six representative scenarios are presented. Test results show that as the number of range differences used increases, or the distance that the observer travels between received beacon signals increases, the accuracy of the estimated position improves. Also, tests show that accuracy of the estimated beacon position is directly related to the accuracy in which the observer's position is measured. To test the receiver's design specifications, a prototype receiver is built using commonly available components. It is then shown that the prototype receiver meets or exceeds the design specifications. 


\section{Table of Contents}

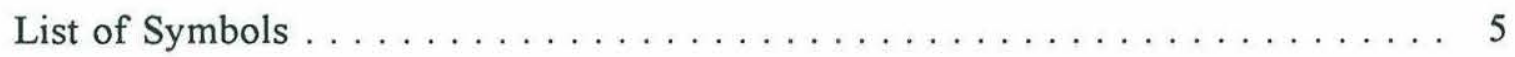

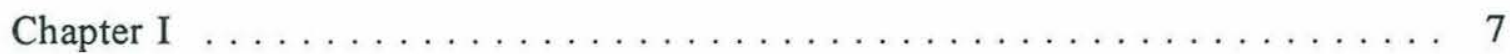

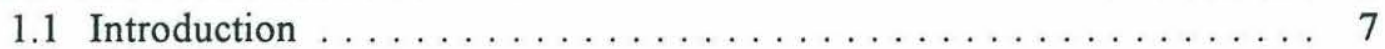

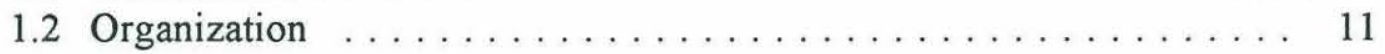

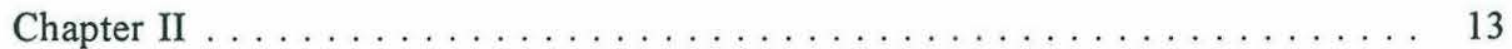

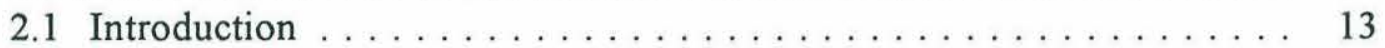

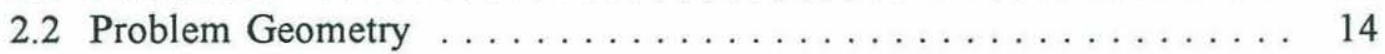

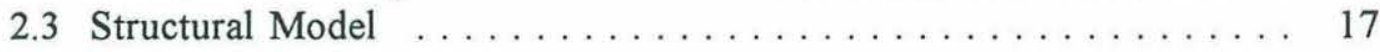

2.4 Measurement Errors . . . . . . . . . . . . . . . . . . . . . 20

2.5 Parameter Estimation Solution - Maximum Likelihood Estimator _ . 23

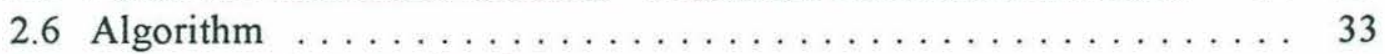

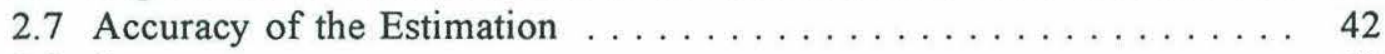

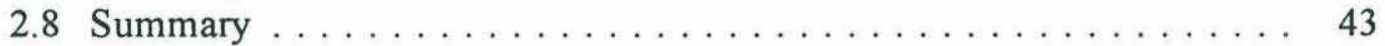

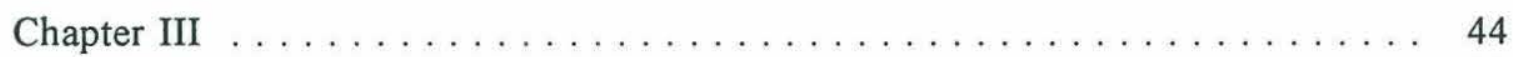

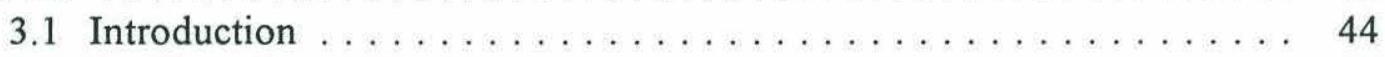

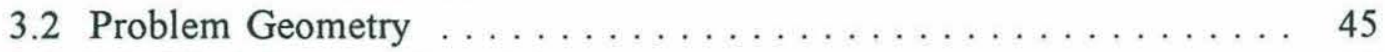

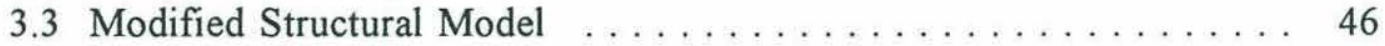

3.4 Maximum Likelihood Estimator Solution . . . . . . . . . . . . . . 48

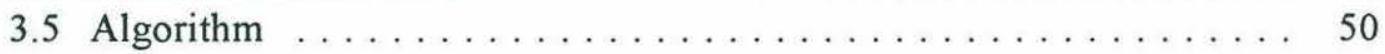

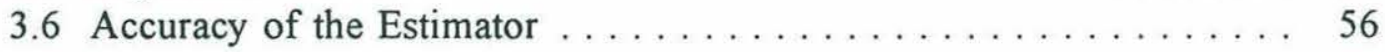

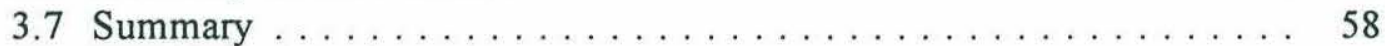

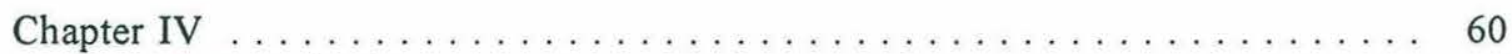

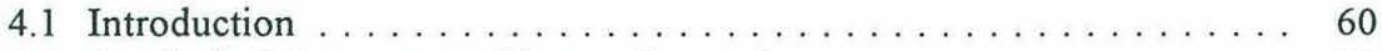

4.2 Synthetic Measurement Vector Generation . . . . . . . . . . 61

4.3 Stationary Acoustic Beacon . . . . . . . . . . . . . . . . . 65

4.3.1 Scenario 1 - Stationary Acoustic Beacon Located at

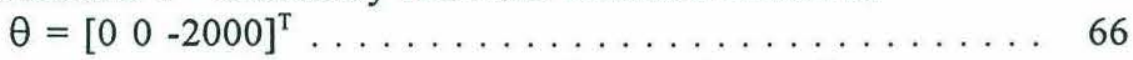

4.3.2 Scenario 2 - Stationary Acoustic Beacon Located at

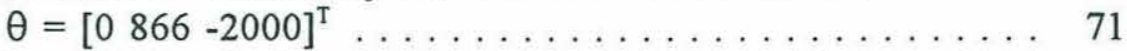

4.3.3 Scenario 3 - Stationary Acoustic Beacon Located at

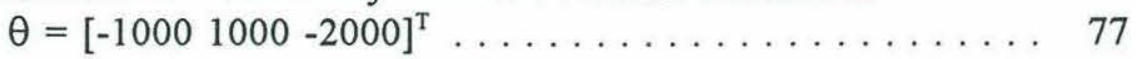

4.3.4 Stationary Acoustic Beacon Summary . . . . . . . . . . . 82

4.4 Moving Acoustic Beacon . . . . . . . . . . . . . . . . 83

4.4.1 Scenario 4 - Moving Acoustic Beacon Located at: $\theta=\left[\begin{array}{llllll}0 & 0 & -2000 & 0.5 & -0.5 & 0.25\end{array}\right]^{\mathrm{T}}$ 
4.4.2 Scenario 5 - Moving Acoustic Beacon Located at: $\theta=\left[\begin{array}{llllll}0 & 866 & -2000 & 0.5 & -0.5 & 0.25\end{array}\right]^{\mathrm{T}} \ldots \ldots \ldots \ldots . \ldots 4$

4.4.3 Scenario 6 - Moving Acoustic Beacon Located at:

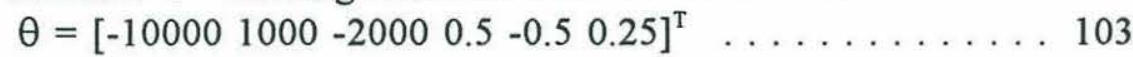

4.4.4 Moving Acoustic Beacon Summary . . . . . . . . . . . 112

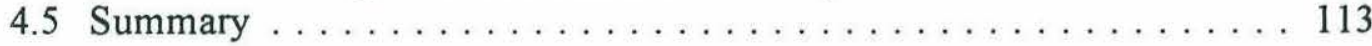

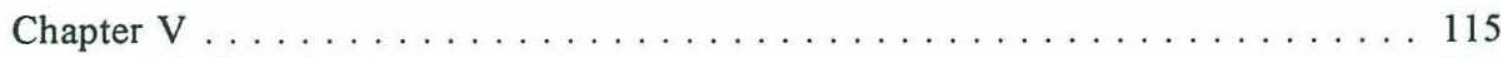

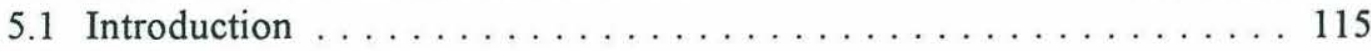

5.2 Design Criterion . . . . . . . . . . . . . . . . . 116

5.3 Hydrophone Model . . . . . . . . . . . . . . . . . . . . . . . . . . . . 125

5.4 Test Receiver Hardware Design . . . . . . . . . . . . . . . . 128

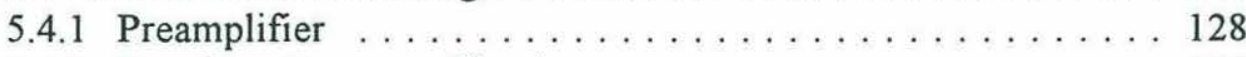

5.4 .2 Envelope Detector Circuit . . . . . . . . . . . . . 135

5.4 .3 Logarithmic Compressor . . . . . . . . . . . . . . . . . 136

$5.4 .468 \mathrm{HC} 11$ Microcontroller . . . . . . . . . . . . . . 138

5.4 .5 Power Supply . . . . . . . . . . . . . . . . . 139

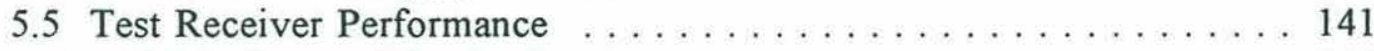

5.5 .1 Preamplifier Performance . . . . . . . . . . . . . . 141

5.5 .2 Envelope Detector . . . . . . . . . . . . . . . 143

5.5 .3 Logarithmic Compressor . . . . . . . . . . . . . . . . . 144

5.6 Conclusions . . . . . . . . . . . . . . . . . . . . . . 145

Chapter VI . . . . . . . . . . . . . . . . . . . . 147

6.1 Observations and Summary . . . . . . . . . . . 147

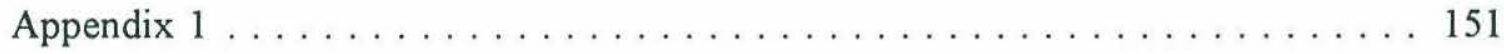

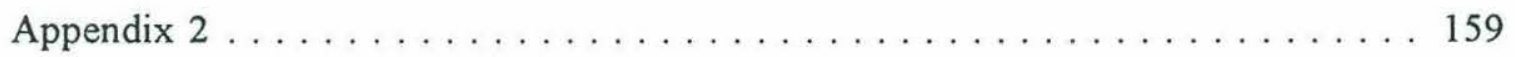

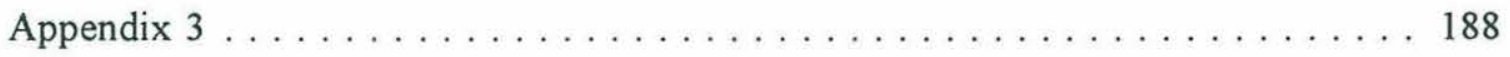

References ............................ 223 


\section{List of Symbols}

$\begin{array}{ll}C & \text { speed of sound in sea water } \\ \mathrm{e}_{\mu} & \text { residual error vector } \\ \mathrm{e}_{\mu}^{*} & \text { final residual error vectors } \\ \varepsilon_{\mathrm{i}} & \text { tolerance factor used to stop iterating } \\ \mathrm{g}\left(\mathrm{W}_{\mu}, \theta\right) & \text { structural model of the parameter estimation problem } \\ \mathbf{G}(\mathbf{W}, \theta) & \text { augmented vector of the structural model } \\ \mathbf{G}_{\mathrm{i}} & \text { augmented vector of the structural model evaluated at }\left(\mathbf{W}^{*}, \theta^{*}\right) \\ \lambda & \text { Lagrange multipliers } \\ \Lambda & \text { Lagrangian function } \\ \mathrm{m}_{\mu} & \text { measurement vector } \\ \mathrm{M} & \text { moment matrix of the final estimated residual vectors } \\ n & \text { number of experiment vectors used to estimate parameter vector } \theta \\ \Phi(\mathbf{W}) & \text { objective function } \\ \theta & \text { parameter vector describing the acoustic beacon's position and velocity. } \\ \theta^{*} & \text { estimated parameter vector } \\ \mathrm{t}_{\mu} & \text { time of receipt of a beacon signal } \\ \mathrm{t}_{\mathrm{s} \mu} & \text { measured synchronized time of receipt } \\ \hat{\mathrm{t}}_{\mathrm{s \mu}} & \text { true synchronized time of receipt } \\ \mathrm{t}_{\mathrm{s} \mu} & \text { measurement errors of the synchronized time of receipt } \\ \mathrm{t}_{\beta} & \text { time that the acoustic beacon transmits a signal } \\ \mathrm{T}_{\mathrm{o}} & \text { time that the acoustic beacon transmits the first signal received } \\ \mathrm{T}_{\mathrm{p}} & \text { pulse repetition interval } \\ \mathrm{T}_{\mathrm{R}} & \text { acoustic travel time of the signal } \\ \mathrm{T}_{\mathrm{s}} & \text { sampling rate of the receiver } \\ \sigma_{\mathrm{t} \mu}^{2} & \text { variance of the synchronized time of receipt measurement error } \\ \sigma_{\mathrm{x} \mu}^{2}, \sigma_{\mathrm{y} \mu}^{2} & \text { variance of the measured ship's position error } \\ \mathrm{V}_{\mu} & \text { covariance matrix of the measurement errors } \\ \mathrm{W}_{\mu} & \text { measured experiment vector } \\ & \end{array}$




$\begin{array}{ll}\hat{\mathrm{w}}_{\mu} & \text { true value of the experiment vector } \\ \tilde{\mathrm{w}}_{\mu} & \text { measurement errors of the experiment vector } \\ \hat{\mathrm{w}}_{\mu}^{*} & \text { estimated true experiment vector } \\ \mathbf{W} & \text { augmented vector of } n \text { experiment vectors } \\ \mathrm{x}_{\mu}, \mathrm{y}_{\mu} & \text { measure location of the receiving ship } \\ \hat{\mathrm{x}}_{\mu}, \hat{\mathrm{y}}_{\mu} & \text { true location of the receiving ship } \\ \tilde{\mathrm{x}}_{\mu}, \tilde{\mathrm{y}}_{\mu} & \text { measurement errors of the location of the receiving ship } \\ \mathrm{x}_{\mathrm{T}}, \mathrm{y}_{\mathrm{T}}, \mathrm{z}_{\mathrm{T}} & \text { the acoustic beacon' position for the stationary case } \\ \mathrm{x}_{0}, \mathrm{y}_{o}, \mathrm{z}_{\mathrm{o}} & \text { the acoustic beacon's initial position for the moving beacon case } \\ \mathrm{x}_{\mathrm{R}}, \mathrm{y}_{\mathrm{R}} & \text { origin of the local Cartesian coordinate system } \\ \mathrm{x}_{\mathrm{B}}, \mathrm{y}_{\mathrm{B}}, \mathrm{z}_{\mathrm{B}} & \text { acoustic beacon position used to generate synthetic measurement vectors }\end{array}$




\section{Chapter I}

\section{Introduction and Problem Statement}

\subsection{Introduction}

There are many scenarios in which passive localization of an acoustic beacon is needed. Techniques like Target Motion Analysis and Bearings Only Ranging can passively estimate an acoustic beacon's position by using received bearing information. These techniques rely upon a multiple sensor receiving array to find the relative bearing between the acoustic beacon and the observer. To estimate the position of the

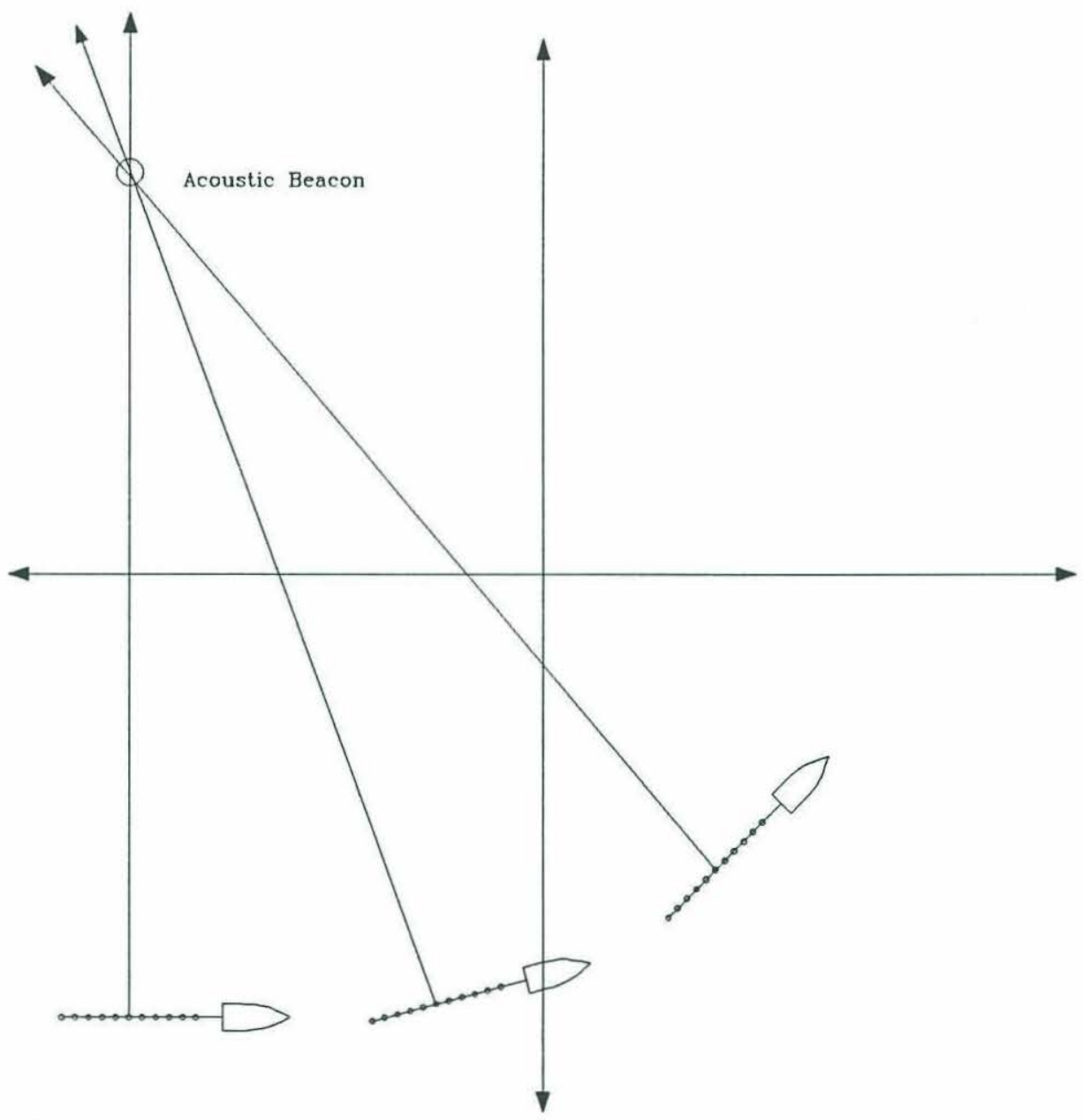

Figure 1-1 
acoustic beacon, bearing lines from several different observation points are combined to form a fix. See figure 1-1.

Recent developments in passive target ranging and tracking which use arrays of only a few elements can also be used to passively localize an acoustic beacon [Allen and King 1988] [Friedlander 1987] [Moose 1987]. These methods rely upon the array to estimate the relative bearing between the acoustic beacon and the observer as well. Statistical and test analyses have shown that the performance of the localization techniques mentioned above improves with increased distance between sensor elements in the receiving array [Moose 1987]. This typically leads to the use of long receiving arrays. The use of long arrays can become a problem if the ship used to deploy the array is small, or if budget constraints limit the size of the array. For these cases, the observer can employ a technique that uses a single omnidirectional sensor to form a synthetic aperture array instead of a conventional receiving array to passively localize the acoustic beacon.

Previous techniques to localize acoustic beacons using a single omnidirectional hydrophone instead of an array use a raster scanning paper recording device to produce a mark on recording paper whenever a beacon signal is received. The scan rate of the paper recorder is matched to the pulse repetition interval of the acoustic beacon. This synchronizes the paper recorder, so that a new line is printed for each pulse repetition interval. The observer can note the relative change in travel time between successive beacon signals by comparing the locations of the corresponding marks on the recording paper. If the observer and the acoustic beacon are stationary, 


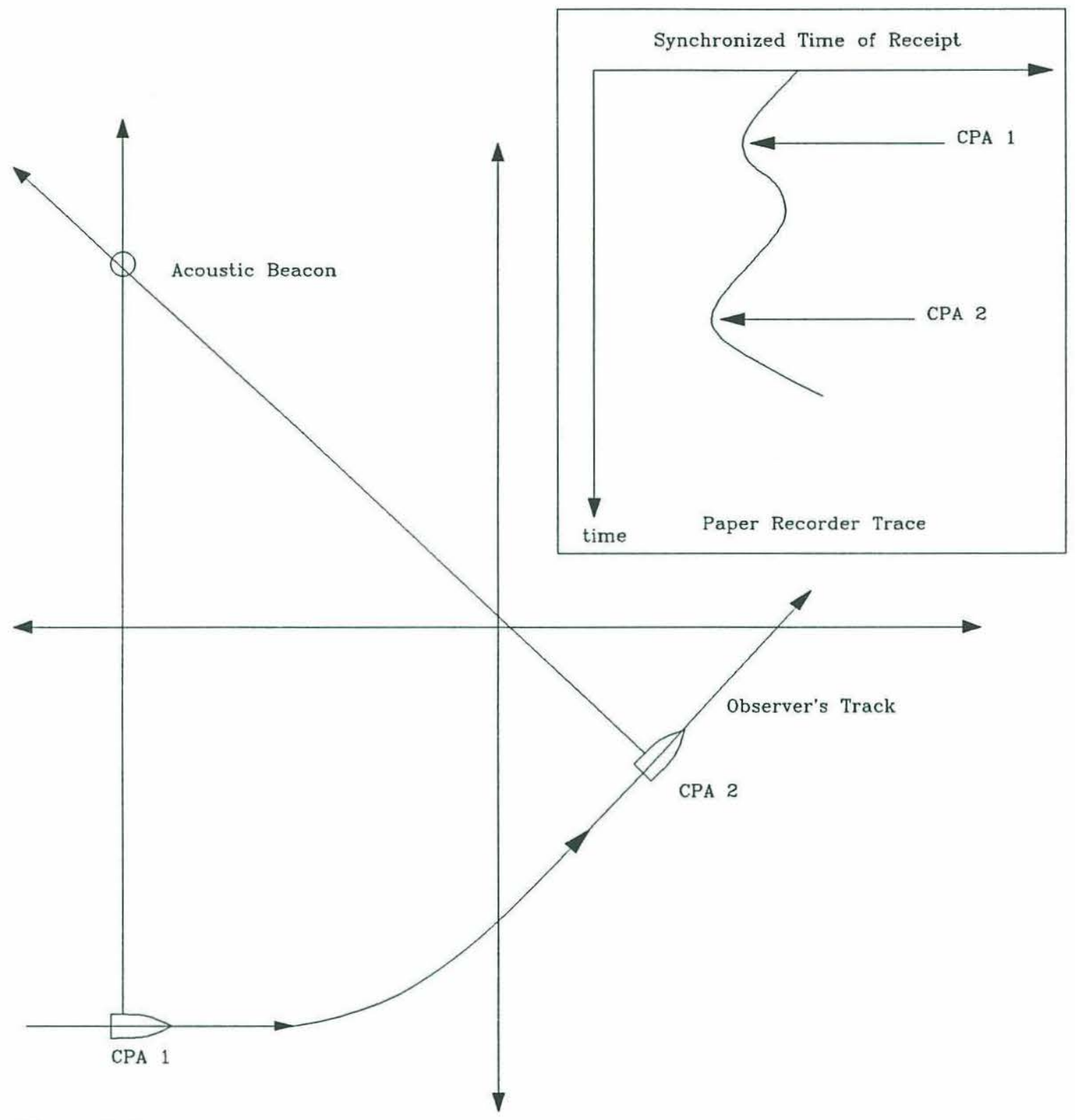

Figure 1-2

the time it takes the acoustic beacon's signal to travel between the beacon and the observer is constant. For this case, the trace made by the paper recorder is a vertical line. As the observer approaches the beacon along a linear path, the range to the beacon, and consequently the time that the beacon signal takes to travel to the 
observer, decreases hyperbolically. The corresponding trace made by the paper recorder is also hyperbolic. The observer can use this relation to fix the acoustic beacon's position. To obtain a line of bearing to the acoustic beacon, the observer watches for the closet point of approach (CPA) to the acoustic beacon by noting the point where the trace produced by the paper recorder changes from negative to positive slope. This corresponds to the transverse axis of the hyperbolic trace and is the point where the range changes from decreasing to increasing. At the CPA, the acoustic beacon is located on a line of bearing perpendicular to the direction of the observer's motion. To estimate the location of the acoustic beacon, the observer must obtain lines of bearing from at least two different directions of motion. See figure 1-2. This method of passively locating acoustic beacons relies heavily upon the operator's skill in visually identifying the closest point of approach from the printed output of the paper recorder. Also, the observer is forced to travel in a straight line for a significant period of time until the CPA can be determined. Other factors that limit the usefulness of the above method are that paper recorders tend to be bulky, awkward to move, and require an adequate supply of expensive recording paper.

This thesis examines the use of a single omnidirectional hydrophone in place of a conventional receiving array to efficiently localize an acoustic beacon using a nonlinear parameter estimation technique. The goal is to develop an inexpensive, easily deployed localization receiver that can interface to a personal computer for data analysis and signal processing. As a backup, this receiver must also perform the functions of the older paper recording system, using the personal computer as the 
display device.

\subsection{Organization}

This thesis is organized into six chapters and three appendices:

- Chapter I contains an introduction to the problem of passively localizing acoustic beacons.

- Chapter II contains the development of a technique to localize a stationary acoustic beacon using a constrained Maximum Likelihood Estimator (MLE).

- Chapter III contains an extension of the solution to the stationary acoustic beacon localization problem that allows for constant velocity, linear motion of the acoustic beacon.

- Chapter IV contains results of Monte Carlo simulations of the localization algorithms developed in chapters II and III. The effects of using different effective sensor spacings and different numbers of experimental data points are explored.

- Chapter V contains design criteria for a receiver to implement the algorithms developed in chapters II and III. A test receiver is designed and built based upon these criteria, and experiments are conducted to measure the actual performance of the receiver.

- Chapter VI contains observations and concluding remarks.

- Appendix 1 contains the schematic diagrams of the receiver designed in chapter V.

- Appendix 2 lists the source code for the $68 \mathrm{HC} 11$ microcontroller used in the 
receiver designed in chapter $\mathrm{V}$.

- Appendix 3 lists the source code for the Macintosh computer used for data analysis and signal processing. 


\section{Chapter II}

\section{Stationary Acoustic Beacon}

\subsection{Introduction}

When the acoustic beacon is stationary, we are interested in locating the acoustic beacon to within a certain error bound as efficiently as possible. To accomplish this we treat the acoustic beacon's location as an unown parameter vector, and formulate the localization problem as a constrained multiple dimension parameter estimation problem. To solve the constrained parameter estimation problem, we first develop a structural model that relates the values of a set of available measurements to the unknown parameter vector. We then define a residual error vector as the difference between the true values and the actual values of the measurements. Assuming the measurement errors are normally distributed, independent random variables, we define the likelihood function for a particular set of measurements as the logarithm of the joint probability density function of the measurements errors, viewed as a function of the residual error vectors. Next, we form a set of equality constraints by evaluating the structural model at the true values of the measurements, and the true value of the parameter vector $\theta$. To find the maximum likelihood estimate (MLE) of the unknown parameter vector, we introduce a vector of Lagrange multipliers, and then define a Lagrangian function as the combination of the likelihood function and the sum of the equality constraints, weighted by the Lagrange multipliers. The maximum likelihood estimate is then found by determining the stationary point of the Lagrangian function. 


\subsection{Problem Geometry}

Consider the acoustic beacon to be at a fixed latitude, longitude, and depth.

Since we are only concerned with locating the acoustic beacon in a small region of the ocean, we approximate the earth as being flat in the region around the beacon's

position and use a Cartesian coordinate system to describe the location of the acoustic beacon. The use of a Cartesian coordinate system does not contribute any significant errors as long as we correct for distortions due to the projection of the acoustic beacon's global coordinates onto the local Cartesian coordinate system. We define a parameter vector $\theta$ as:

$$
\theta=\left[\begin{array}{lll}
x_{T} & y_{T} & z_{T}
\end{array}\right]^{T}
$$

where $\mathrm{x}_{\mathrm{T}}$ represents the east-west position of the acoustic beacon, $\mathrm{y}_{\mathrm{T}}$ represents the north-south position of the acoustic beacon, and $\mathrm{z}_{\mathrm{T}}$ represents the depth of the acoustic beacon in meters relative to the center of the local Cartesian coordinate system. To correct for the distortions caused by projecting the acoustic beacon's global coordinates onto the local Cartesian coordinate system, we introduce a correction factor that is proportional to the cosine of the latitude. We then relate $\theta$ to the acoustic beacon's global position by:

$$
\theta=\left[\begin{array}{l}
x_{T} \\
y_{T} \\
z_{T}
\end{array}\right]=\left[\begin{array}{c}
\text { degrees longitude } \cdot \frac{60 \mathrm{Nm}}{1^{\circ}} \cdot \frac{1853.2 \mathrm{~m}}{1 \mathrm{Nm}} \cdot \cos (\text { degrees latitude })-x_{R} \\
\text { degrees latitude } \cdot \frac{60 \mathrm{Nm}}{1^{\circ}} \cdot \frac{1853.2 \mathrm{~m}}{1 \mathrm{Nm}}-y_{R} \\
- \text { depth }
\end{array}\right]
$$


where $x_{R}$ and $y_{R}$ define an arbitrary reference point on the surface of the ocean which defines the origin of the local Cartesian coordinate system. In equation (2-2) we assume that the acoustic beacon's latitude and longitude are given in degrees.

Similarly, we consider an observer on a receiving ship in the general location of the acoustic beacon. We define a measurement vector $\mathrm{m}_{\mu}$ as:

$$
m_{\mu}=\left[\begin{array}{lll}
x_{\mu} & y_{\mu} & t_{\mu}
\end{array}\right]^{T}
$$

where $x_{\mu}$ and $y_{\mu}$ are the receiving ship's coordinates on the same local Cartesian coordinate system defined for the acoustic beacon, and $t_{\mu}$ is the time that the observer's position $\mathrm{x}_{\mu}, \mathrm{y}_{\mu}$ was obtained. We use the same relation described in equation (2-2) to convert the receiving ship's global coordinates to local Cartesian coordinates. The measurement vector $m_{\mu}$ is then given by:

$$
m_{\mu}=\left[\begin{array}{c}
x_{\mu} \\
y_{\mu} \\
t_{\mu}
\end{array}\right]=\left[\begin{array}{c}
\text { degrees longitude } \cdot \frac{60 \mathrm{Nm}}{1^{\circ}} \cdot \frac{1853.2 \mathrm{~m}}{1 \mathrm{Nm}} \cdot \cos (\text { degrees latitude })-x_{R} \\
\text { degrees latitude } \cdot \frac{60 \mathrm{Nm}}{1^{\circ}} \cdot \frac{1853.2 \mathrm{~m}}{1 \mathrm{Nm}}-y_{R} \\
t_{\mu}
\end{array}\right]
$$

Again, we assume that the receiving ship's latitude and longitude are given in degrees.

Figure 2-1 summarizes the problem geometry.

Now let us assume that the acoustic beacon transmits a signal at a regular interval, and that at time $t_{\mu}$ the observer receives one of the signals from the acoustic beacon. The time $t_{\mu}$ can be related to the time that the signal took to reach the 


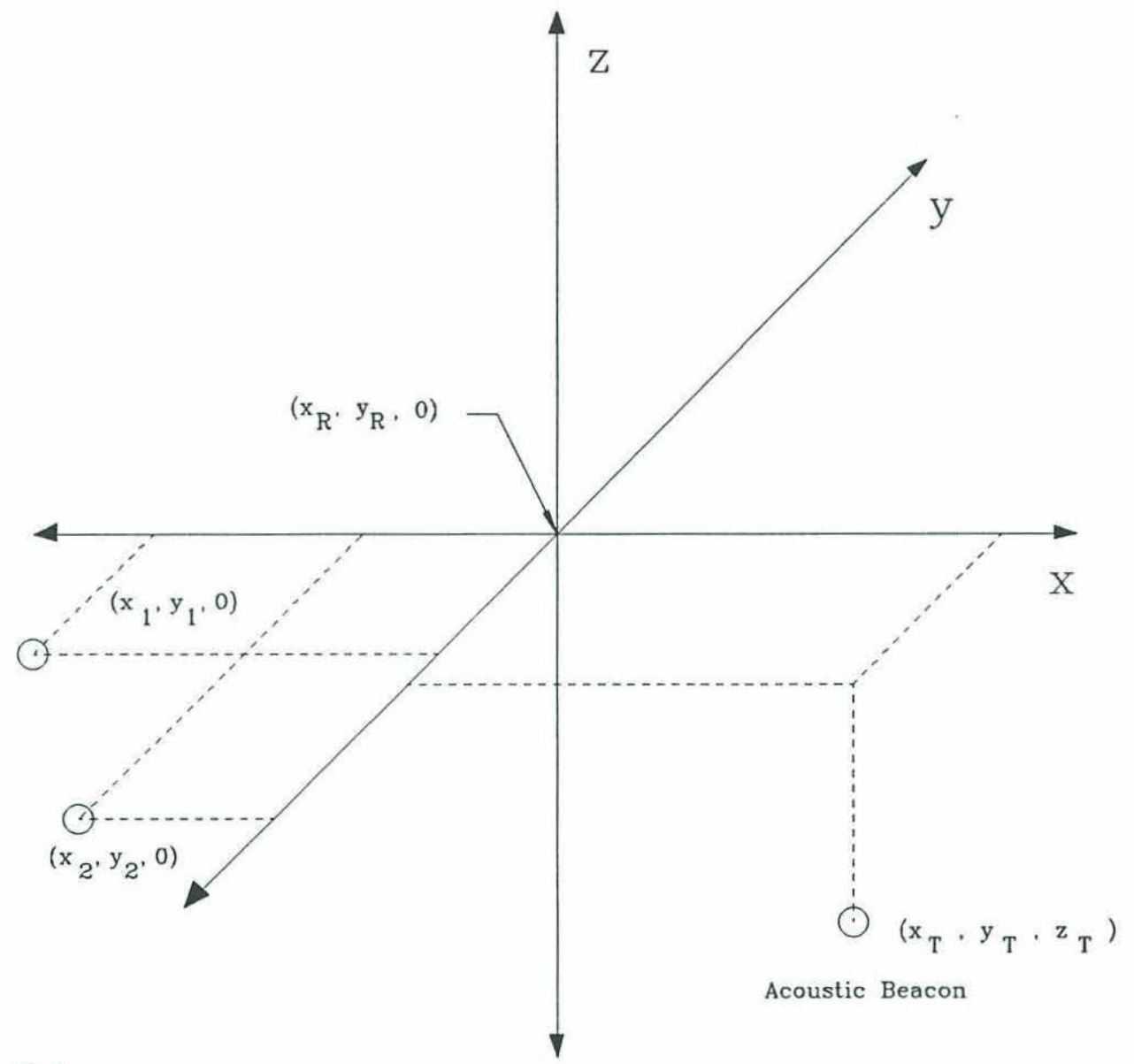

Figure 2-1

observer by:

$$
t_{\mu}=T_{R}+i \cdot T_{p}+T_{o}
$$

Here $T_{R}$ represents the acoustic travel time of the signal, $T_{0}$ is the time that the acoustic beacon transmitted the first signal, $i$ is the number of pulse repetition intervals between when the first signal was transmitted and when the current signal was transmitted, and $\mathrm{T}_{\mathrm{p}}$ represents the pulse repetition interval. The product $i \cdot \mathrm{T}_{\mathrm{p}}$ represents the time after $T_{0}$ that the acoustic beacon transmitted the current received signal. It is 
assumed that the observer knows the pulse repetition interval of the acoustic beacon. The observer can therefore remove the dependance upon $i \cdot \mathrm{T}_{\mathrm{p}}$ by counting the number of signals received $(i)$, then subtracting $i \cdot \mathrm{T}_{\mathrm{p}}$ from equation (2-5). This synchronizes the observer's receiver with the pulse repetition interval of the acoustic beacon. We define the synchronized time of receipt as the time of receipt $t_{\mu}$ corrected to eliminate the pulse repetition interval of the acoustic beacon as:

$$
t_{s \mu} \equiv t_{\mu}-i \cdot T_{p}=T_{R}+T_{o}
$$

and subsequently redefine the measurement vector $\mathrm{m}_{\mu}$ as:

$$
m_{\mu}=\left[\begin{array}{lll}
x_{\mu} & y_{\mu} & t_{s \mu}
\end{array}\right]^{T}
$$

The difficulty in passively ranging an acoustic beacon arises because the observer does not know $T_{0}$, the time the acoustic beacon started to transmit. In active ranging systems, the observer can measure $T_{R}$ directly by transmitting a signal at a known time $T_{0}$, and measuring the time that the corresponding reflected signal from a target is received. In passive ranging systems, the observer does not know when the beacon signal was transmitted and therefore the observer has no time reference $T_{0}$. Consequently the observer has no way to directly calculate $T_{R}$. In order to passively range a target, the observer must eliminate the dependence on the absolute time reference $T_{0}$.

\subsection{Structural Model}

In order to proceed, we must develop a model which relates the measurement vector $\mathrm{m}_{\mu}$, and the unknown parameter vector $\theta$. We define a function $\mathbf{f}\left(\mathbf{m}_{\mu}, \theta\right)$ 
which relates the distance between the acoustic beacon's position $\theta$ and the receiving ship's position $x_{\mu}, y_{\mu}$ to the synchronized time of receipt $t_{s \mu}$ as:

$$
f\left(m_{\mu}, \theta\right) \equiv \sqrt{\left(x_{\mu}-x_{T}\right)^{2}+\left(y_{\mu}-y_{T}\right)^{2}+z_{T}^{2}}-\tau\left(C, t_{s \mu}\right)
$$

where $\tau\left(\mathbf{C}, \mathbf{t}_{\mathrm{su}}\right)$ is a function that relates the speed of sound in seawater to the measured time of receipt, synchronized to the pulse repetition interval of the acoustic beacon. It is assumed that the receiving ship is on the surface of the ocean, or equivalently $z_{\mu}$ is zero. Using a homogeneous ocean model and a direct path propagation mode for simplicity, we can define $\tau\left(\mathbf{C}, \mathbf{t}_{\mathrm{sp}}\right)$ from equation (2-6) as:

$$
\tau\left(C, t_{s \mu}\right)=C \cdot T_{R}=C \cdot t_{s \mu}-C \cdot T_{o}
$$

Substituting equation (2-9) into equation (2-8) yields:

$$
f\left(m_{\mu}, \theta\right)=\sqrt{\left(x_{\mu}-x_{T}\right)^{2}+\left(y_{\mu}-y_{T}\right)^{2}+z_{T}^{2}}-\left(C \cdot t_{s \mu}-C \cdot T_{o}\right)
$$

It is apparent from equation (2-10) that we can not eliminate the dependence upon $T_{0}$ with only one received signal from the acoustic beacon. One way around this problem is to compare the time of receipt and positions from two separate received signals. This is commonly referred to as range difference, or time difference ranging. We define a new function $\mathbf{g}\left(\mathbf{m}_{1}, \mathbf{m}_{2}, \theta\right)$ as:

$$
g\left(m_{1}, m_{2}, \theta\right) \equiv f\left(m_{1}, \theta\right)-f\left(m_{2}, \theta\right)
$$

Substituting equation (2-10) into equation (2-11) with $m_{1}$ for the first received signal and $\mathrm{m}_{2}$ for the second received signal we have: 


$$
\begin{aligned}
g\left(m_{1}, m_{2}, \theta\right)= & \sqrt{\left(x_{1}-x_{T}\right)^{2}+\left(y_{1}-y_{T}\right)^{2}+z_{T}^{2}}+\ldots \\
& -\sqrt{\left(x_{2}-x_{T}\right)^{2}+\left(y_{2}-y_{T}\right)^{2}+z_{T}^{2}}+\ldots \\
& -C \cdot\left(t_{s 1}-t_{s 2}\right)
\end{aligned}
$$

The first two terms of the function $\mathbf{g}\left(\mathbf{m}_{1}, \mathbf{m}_{2}, \theta\right)$ represent the slant range between the acoustic beacon and the receiving ship at the two separate times that a beacon signal is received. By subtracting one range from the other, we are left with the distance that the observer moved radially, relative to the beacon, between the times $t_{s 1}$ and $t_{s 2}$. This is known as the range difference between the two points given by $m_{1}$ and $m_{2}$. The last term of the function $\mathbf{g}\left(\mathbf{m}_{1}, \mathbf{m}_{2}, \theta\right)$ represent the range difference between points $m_{1}$ and $\mathrm{m}_{2}$ calculated from the time difference. From the last term of $\mathbf{g}\left(\mathbf{m}_{1}, \mathbf{m}_{\mathbf{2}}, \theta\right)$ we see that the dependence upon $\mathrm{T}_{0}$ is eliminated, and we are left with a model that relies on measuring the time of receipt, synchronized to the pulse repetition interval of the acoustic beacon, and the location of the receiving ship at the time of receipt.

To simplify notation, we define an experiment to be the augmentation of two measurement vectors $m_{1}$ and $m_{2}$, and use the notation:

$$
w_{\mu}=\left[\begin{array}{l}
m_{1} \\
m_{2}
\end{array}\right]=\left[\begin{array}{llllll}
x_{1} & y_{1} & t_{s 1} & x_{2} & y_{2} & t_{s 2}
\end{array}\right]^{T}
$$

We then redefine the equation (2-12) as a scaler valued function of the experiment vector $\mathrm{w}_{\mu}$ and the acoustic beacon location vector $\theta$ :

$$
g\left(w_{\mu}, \theta\right)=g\left(m_{1}, m_{2}, \theta\right)
$$


We denote the function $\mathbf{g}\left(\mathbf{w}_{\mu}, \theta\right)$ as the structural model of the parameter estimation problem. Ideally, if the measurements have no errors and $\tau\left(\mathbf{C}, \mathbf{t}_{\mathrm{sp}}\right)$ is the correct propagation model, $\mathbf{g}\left(\mathbf{w}_{\mu}, \theta\right)$ equals zero at the true value of the parameter vector $\theta$. The estimation problem is then to determine the value of the parameter vector $\theta$ that minimizes the value of the structural model given experiment vectors which contain errors.

\subsection{Measurement Errors}

The structural model describes the ideal situation - perfect measurements, free from any errors. In reality both the synchronized time of receipt $t_{s \mu}$ and the positions $\mathrm{x}_{\mu}, \mathrm{y}_{\mu}$ have errors associated with the measurement process. We can define the actual measurements as the sum of the true value and a random error component:

$$
w_{\mu}=\hat{w}_{\mu}+\tilde{w}_{\mu}=\left[\begin{array}{c}
\hat{x}_{1}+\tilde{x}_{1} \\
\hat{y}_{1}+\tilde{y}_{1} \\
\hat{t}_{s 1}+\tilde{t}_{s 1} \\
\hat{x}_{2}+\tilde{x}_{2} \\
\hat{y}_{2}+\tilde{y}_{2} \\
\hat{t}_{s 2}+\tilde{t}_{s 2}
\end{array}\right]
$$

where $\hat{x}_{\mu}, \hat{y}_{\mu}, \hat{t}_{s \mu}$, and $\hat{w}_{\mu}$ represent the true values of the measurements, and $\tilde{x}_{\mu}, \tilde{y}_{\mu}$, $\mathfrak{f}_{s \mu}$, and $\tilde{\mathrm{w}}_{\mu}$ represent the random errors associated with the measurement process.

With the assumption of a homogeneous, direct path propagation mode, and since the time of receipt will be determined by looking at the output of a simple digital peak detector, the errors in measuring $\hat{t}_{s \mu}$ arise mainly from the sampling of the received signal. Because we use a simple peak detector to measure the time of 
receipt, we can only estimate the true time of receipt to within one sampling interval if we sample the received signal every millisecond, then we can only know the time of receipt to within 1 millisecond. This quantization of the time base causes the measurement errors to have a uniform distribution centered around the true value $\hat{t}_{\mathrm{s} \mu}$ with a variance given by:

$$
\sigma_{t_{\mu}}^{2}=\frac{T_{s}^{2}}{12}
$$

where $T_{S}$ is the sampling interval in seconds. For the sake of computational efficiency and mathematical tractability, we would like to model the measurement errors of $t_{s \mu}$ as zero mean, normally distributed random variables. If we consider that the structural model is not exact (a far more complex function $\tau\left(\mathbf{C}, \mathbf{t}_{\mathrm{s} \mu}\right)$ is needed to have an exact structural model), and that there will be errors due to factors such as dispersion and multipath interference, we can lump the modeling errors of $\tau\left(\mathbf{C}, \mathbf{t}_{\mathbf{s p}}\right)$ together with the quantization errors in the time $t_{s \mu}$. Although not strictly rigorous, this allows us to simplify the development of the solution to the localization problem by assuming the measurement errors of $t_{s \mu}$ are zero mean, normally distributed random variables, with a variance approximated by $\sigma_{t \mu}{ }^{2}$. Also, since the measurement of the time of receipt of one signal is not effected by the measurement of any other time of receipt, we can take the errors in measuring $t_{s \mu}$ as independent between $t_{s 1}$ and $t_{s 2}$, and also independent between experiments $\mathrm{w}_{\mu}$.

Errors in measuring the ship's position at the time of receipt of the beacon signal are due to the accuracy of the system used to obtain the ship's position. To 
obtain the ship's location, a Global Positioning System (GPS) receiver will be used. Other navigation systems such as Loran, Omega, or even Inertial Navigation systems could be used, however the price and accuracy of the GPS system make it the natural choice. Currently, inexpensive commercial C/A code GPS receivers are available with published errors on the order of 25 meters rms in latitude and longitude. The more accurate Differential GPS and P code receivers are not readily available at this time.

We will assume that the errors of the GPS positional data are normally distributed, and independent in latitude and longitude. This assumption is unlikely to cause much harm as long as the true probability distribution of the errors have smooth tails [Bard 1974].

With these arguments in mind, and for the sake of computational efficiency and mathematical tractability, we model the measurement errors as zero mean, normally distributed, independent random variables. We take the covariance matrix of the measurement errors as:

$$
V_{\mu}=\left[\begin{array}{cccccc}
\sigma_{x_{\mu}}{ }^{2} & 0 & 0 & 0 & 0 & 0 \\
0 & \sigma_{y_{\mu}}{ }^{2} & 0 & 0 & 0 & 0 \\
0 & 0 & \sigma_{t_{\mu}}{ }^{2} & 0 & 0 & 0 \\
0 & 0 & 0 & \sigma_{x_{\mu}}{ }^{2} & 0 & 0 \\
0 & 0 & 0 & 0 & \sigma_{y_{\mu}}{ }^{2} & 0 \\
0 & 0 & 0 & 0 & 0 & \sigma_{t_{\mu}}{ }^{2}
\end{array}\right]
$$

Where $\sigma_{\mathrm{t} \mu}{ }^{2}$ is the variance given by equation (2-16), $\sigma_{\mathrm{x}}{ }^{2}$ is the variance of the GPS longitude error in meters, and $\sigma_{y}{ }^{2}$ is the variance of the GPS latitude error in meters. 


\subsection{Parameter Estimation Solution - Maximum Likelihood Estimator}

To solve for the parameter vector $\theta$ we would normally use a weighted least square approach. However, because of the nonlinearities in the structural model $\mathbf{g}\left(\mathbf{w}_{\mu}, \theta\right)$, and because all of the measurements have errors, the weighted least squares method does not work well. To demonstrate why the normal weighted least squares method does not work well, we look at the contours of the $\chi^{2}$ merit function:

$$
\chi^{2}=\sum_{i=1}^{n}\left(\frac{g\left(w_{\mu}, \theta\right)}{\sigma_{t_{\mu}}}\right)^{2}
$$

If the structural model is truly exact, and there are no measurement errors in $\mathrm{w}_{\mu}$, $\mathbf{g}\left(\mathbf{w}_{\mu}, \theta\right)$ and $\chi^{2}$ are equal to zero for the true value of $\theta$. As the value of $\theta$ in equation (2-18) moves farther away from the true value of $\theta$, the $\chi^{2}$ function increases. Figure 2-2 shows the contours of the $\chi^{2}$ function for the true values of the measurements $\mathrm{w}_{\mu}$, where the acoustic beacon is located at $\mathrm{x}_{\mathrm{T}}=-1000, \mathrm{y}_{\mathrm{T}}=1000$, and $z_{\mathrm{T}}=-1000$. From Figure 2-2 we see that the contours of the $\chi^{2}$ merit function that encircle the true position of the acoustic beacon are highly eccentric. This eccentricity leads to large variances in the location estimate along the major axis of the ellipse. With errors in all of the measurements, this in turn produces errors in the parameter estimates that are unacceptable.

To overcome the difficulties in solving the parameter estimation problem with a nonlinear structural model and errors in all of the measurements, we treat the problem as a constrained minimization problem. Our goal is to estimate the true values of the measurements $\mathrm{w}_{\mu}$ given a set of $n$ equality constraints which we obtain by setting the 


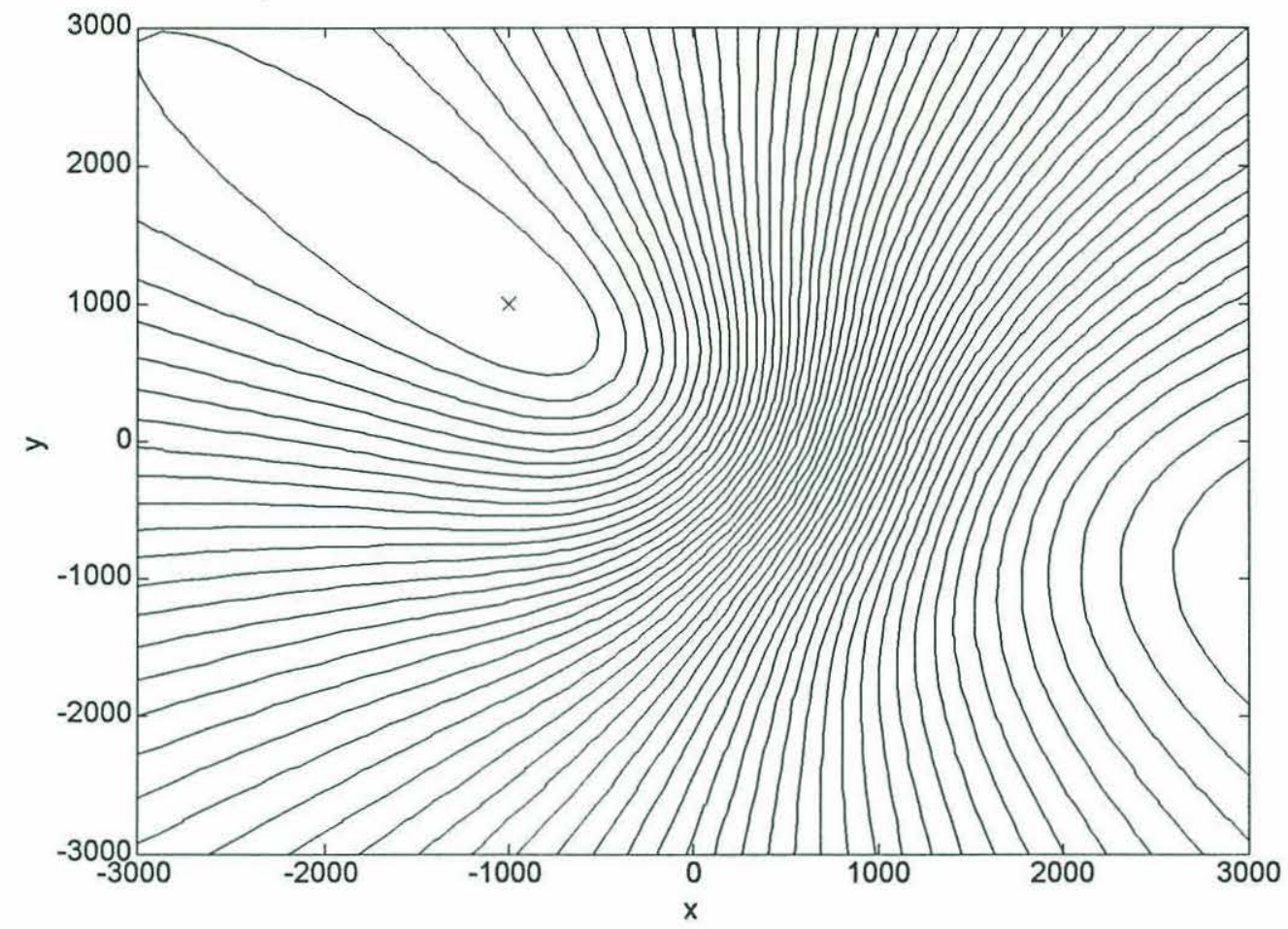

Figure 2-2 Chi Square Contours for the Normal Weighted Least Squares Method

structural model $\mathbf{g}\left(\mathbf{w}_{\mu}, \theta\right)$ equal to zero at the estimated true values of the measurements and the estimated parameter vector $\theta$. Because of the measurement errors, we must modify the structural model to indicate the dependence upon the true values of $\mathrm{w}_{\mu}$ :

$$
\begin{aligned}
g\left(\hat{w}_{\mu}, \theta\right)= & \sqrt{\left(\hat{x}_{1}-x_{T}\right)^{2}+\left(\hat{y}_{1}-y_{T}\right)^{2}+z_{T}^{2}}+\ldots \\
& -\sqrt{\left(\hat{x}_{2}-x_{T}\right)^{2}+\left(\hat{y}_{2}-y_{T}\right)^{2}+z_{T}^{2}}+\ldots \\
& -C \cdot\left(\hat{t}_{s 1}-\hat{t}_{s 2}\right)
\end{aligned}
$$

Next we define a residual vector as the difference between the true value and the observed value of the measurements: 


$$
e_{\mu}\left(\hat{w}_{\mu}\right)=\hat{w}_{\mu}-w_{\mu}
$$

We also define a composite experiment vector $\mathbf{W}$ as the augmentation of $n$ experiment vectors $\mathrm{w}_{\mu}$ :

$$
W=\left[\begin{array}{llll}
w_{1}^{T} & w_{2}^{T} & \ldots & w_{n}^{T}
\end{array}\right]^{T}
$$

With the assumption that the measurement errors are normally distributed random variables, independently distributed between experiments, and with the further assumption that the covariance matrix is the same for each experiment, the likelihood function takes the form:

$$
\log (L(\hat{W}, V))=-(n / 2) \log (\operatorname{det} V)-\frac{1}{2} \cdot \sum_{\mu=1}^{n} e_{\mu}^{T} V^{-1} e_{\mu}
$$

where $n$ is the number of experiment vectors $\mathrm{w}_{\mu}$ used in the estimation problem [Bard 1974]. The maximum likelihood estimate is found by determining the value of $\theta^{*}$, and the value of $\mathrm{w}_{\mu}^{*}$ for each of the $n$ experiments, which minimizes $\log (\mathrm{L})$ while satisfying the $n$ equality constraints imposed by the structural model. The terms $\mathrm{w}_{\mu}^{*}$ and $\theta^{*}$ represent the maximum likelihood estimate of the true values of $\mathrm{w}_{\mu}$ and $\theta$.

To find the maximum likelihood estimate given the $n$ equality constraints imposed by the structural model, we introduce an $n$-dimensional vector of Lagrange multipliers $\lambda_{\mu}$ for each experiment. We then form the Lagrangian function:

$$
\Lambda\left(\hat{W}, \theta, V, \lambda_{1}, \ldots \lambda_{n}\right) \equiv \log (L(\hat{W}, V))+\sum_{\mu=1}^{n} \lambda_{\mu} \cdot g\left(\hat{w}_{\mu}, \theta\right)
$$


The solution to the estimation problem will be found at a stationary point of the Lagrangian $\Lambda\left(\mathrm{W}, \theta, \mathrm{V}, \lambda_{1}, \ldots, \lambda_{\mu}\right)$ [Bard 1974].

To demonstrate the benefits of using this approach, we look at the contours of the Lagrangian function given by equation (2-23). Using the algorithm developed in section 2.6, figure 2-3 shows the contours of the Lagrangian function obtained by using the same experiment vectors used to generate the contours of the normal weighted least squares method.

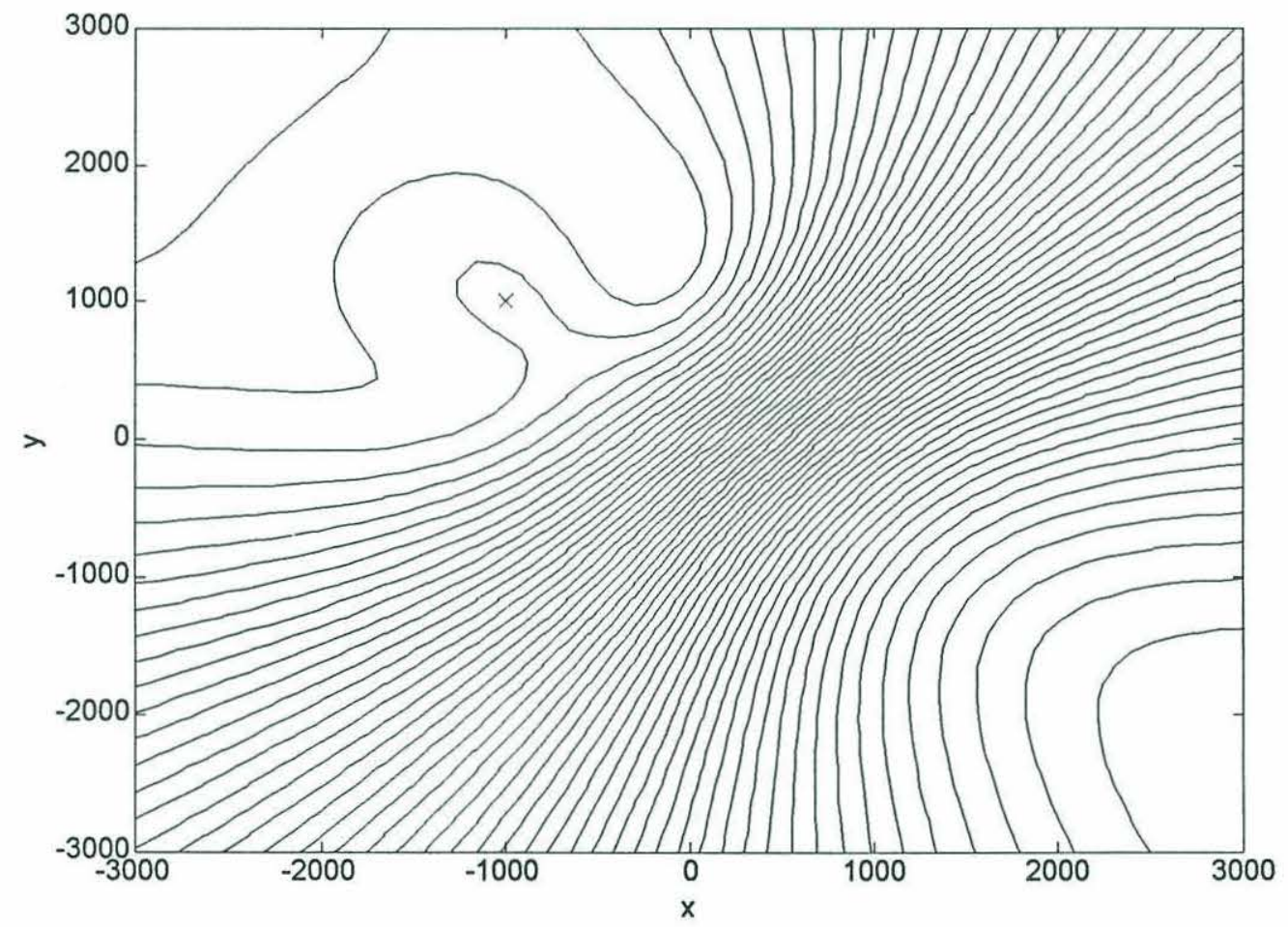

Figure 2-3 Contours of the Lagrangian Function $\Lambda$

From figure 2-3 we see that the contours that surround the true position of the acoustic beacon are much less eccentric then the contours of the normal weighted least squares method. Therefore, we do not have the large variances associated with the major axis 
of the normal weighted least squares method, and the estimate of the parameter vector $\theta$ will be more accurate.

To solve the maximum likelihood estimator problem given the equality constraint of the structural model we use an iterative method suggested by Deming as described by Bard [Bard 1974]. We define the parameter vector $\theta_{\mathrm{i}}^{*}$ as the estimate of the acoustic beacon's location at the $i$ th iteration:

$$
\theta_{i}^{*}=\left[\begin{array}{lll}
x_{T_{i}}^{*} & y_{T_{i}}^{*} & z_{T_{i}}^{*}
\end{array}\right]^{T}
$$

We also define the estimated true experiment vector at the $i$ th iteration as:

$$
\hat{w}_{\mu_{i}}^{*}=\left[\begin{array}{llllll}
\hat{x}_{1_{i}}^{*} & \hat{y}_{1_{i}}^{*} & \hat{t}_{s 1_{i}}^{*} & \hat{x}_{2_{i}}^{*} & \hat{y}_{2_{i}}^{*} & \hat{t}_{s 2_{i}}^{*}
\end{array}\right]^{T}
$$

and the corresponding composite experiment vector:

$$
\hat{W}_{i}^{*}=\left[\begin{array}{llll}
\hat{w}_{1}^{*^{T}}{ }_{i} & \hat{w}_{2}^{*^{T}}{ }_{i} & \ldots & \hat{w}_{n}^{*^{*}}{ }_{i}
\end{array}\right]^{T}
$$

The vector $\hat{\mathbf{W}}_{\mathrm{i}}^{*}$ is the augmentation of the $n$ estimated true experiment vectors $\hat{\mathbf{w}}_{\mu}^{*}$ at the $i$ th iteration. We then define a composite vector valued function $G\left(\mathbf{W}_{i}^{*}, \theta_{i}^{*}\right)$ where the $j$ th component of $\mathrm{G}\left(\hat{\mathbf{W}}_{\mathrm{i}}^{*}, \theta_{\mathrm{i}}^{*}\right)$ is the structural model evaluated at $\left(\hat{\mathrm{w}}_{\mu}^{*}, \theta_{\mathrm{i}}^{*}\right)$ :

$$
G\left(\hat{W}_{i}^{*}, \theta_{i}^{*}\right)=G_{i}=\left[g\left(\hat{w}_{1_{i}}^{*}, \theta_{i}^{*}\right) g\left(\hat{w}_{2_{i}}^{*}, \theta_{i}^{*}\right) \quad \ldots g\left(\hat{w}_{n_{i}}^{*}, \theta_{i}^{*}\right)\right]^{T}
$$

Next we define an objective function as the weighted least squares sum of the 
residuals given by equation (2-20). At the $i$ th iteration the objective function is:

$$
\Phi\left(\hat{W}_{i}^{*}\right)=\frac{1}{2} \cdot \sum_{\mu=1}^{n}\left(\hat{w}_{\mu_{i}}^{*}-w_{\mu}\right)^{T} V_{\mu}^{-1}\left(\hat{w}_{\mu_{i}}^{*}-w_{\mu}\right)
$$

This is the same form as the last term in equation (2-22). To solve for the Maximum Likelihood Estimate, the objective is to find the $\mathbf{W}_{\mathrm{i}}^{*}$ which minimizes $\log (\mathrm{L})$, or equivalently, $\Phi\left(\hat{\mathbf{W}}_{\mathrm{i}}^{*}\right)$ subject to the constraints $\mathrm{G}\left(\hat{\mathbf{W}}_{\mathrm{i}}^{*}, \theta_{\mathrm{i}}^{*}\right)=0$. We note that now the unknown parameters that we are estimating are the acoustic beacon's location as well as the true values of all the measurements.

To develop a solution, we define the following terms:

$$
\begin{gathered}
\boldsymbol{q}_{i} \equiv \partial \Phi\left(\hat{W}_{i}^{*}\right) / \partial \hat{W} \\
\boldsymbol{H}_{i} \equiv \partial^{2} \Phi\left(\hat{W}_{i}^{*}\right) / \partial \hat{W} \partial \hat{W} \\
\boldsymbol{A}_{i} \equiv \partial G\left(\hat{W}_{i}^{*}, \theta_{i}^{*}\right) / \partial \hat{W} \\
\boldsymbol{B}_{i} \equiv \partial G\left(\hat{W}_{i}^{*}, \theta_{i}^{*}\right) / \partial \theta
\end{gathered}
$$

Next we expand the objective function in a Taylor series around the current estimate of $\mathbf{W}$ and $\theta$. Keeping only up to the second order terms we have:

$$
\tilde{\Phi}_{i}(\delta \hat{W}) \equiv \Phi_{i}+\boldsymbol{q}_{i}^{T} \delta \hat{W}+\frac{1}{2} \delta \hat{W}^{T} \boldsymbol{H}_{i} \delta \hat{W}
$$

Now we expand the structural model in a Taylor series around the current estimate of W and $\theta$. Keeping only the first order terms we have: 


$$
\tilde{G}_{i}(\delta \hat{W}, \delta \theta) \equiv G_{i}+\boldsymbol{A}_{i} \delta \hat{W}+\boldsymbol{B}_{i} \delta \theta
$$

To find the maximum likelihood estimate we now want to find the $\delta W$ and $\delta \theta$ that minimize $\tilde{\Phi}_{\mathrm{i}}$ while at the same time satisfies the structural model constraints $\tilde{G}_{\mathrm{i}}=0$. To do this we introduce the vector of Lagrange multipliers $\lambda$ and look for the stationary point of the Lagrangian:

$$
\Lambda_{i}\left(\delta \hat{W}, \delta \theta, \lambda_{i}\right) \equiv \tilde{\Phi}_{i}(\delta \hat{W})+\lambda_{i}^{T} \tilde{G}_{i}
$$

Forming the normal equations of the Lagrangian and using equations (2-33) and (2-34) we have:

$$
\begin{gathered}
\partial \Lambda_{i}\left(\delta \hat{W}, \delta \theta, \lambda_{i}\right) / \partial(\delta \hat{W})=\boldsymbol{q}_{i}+\boldsymbol{H}_{i} \delta \hat{W}+\boldsymbol{A}_{i}^{T} \lambda_{i}=0 \\
\partial \Lambda_{i}\left(\delta \hat{W}, \delta \theta, \lambda_{i}\right) / \partial \lambda_{i}=G_{i}+\boldsymbol{A}_{i} \delta \hat{W}+\boldsymbol{B}_{i} \delta \theta=0 \\
\partial \Lambda_{i}\left(\delta \hat{W}, \delta \theta, \lambda_{i}\right) / \partial(\delta \theta)=\boldsymbol{B}_{i}^{T} \lambda_{i}=0
\end{gathered}
$$

From equation (2-36):

$$
\delta \hat{W}=-\boldsymbol{H}_{i}^{-1} \cdot\left(\boldsymbol{q}_{i}+\boldsymbol{A}_{i}^{T} \lambda_{i}\right)
$$

Substituting equation (2-39) into equation (2-37) we have:

$$
G_{i}-A_{i} H_{i}^{-1} q_{i}-A_{i} H_{i}^{-1} A_{i}^{T} \lambda_{i}+B_{i} \delta \theta=0
$$

Solving for $\lambda_{i}$ :

$$
\lambda_{i}=\left(A_{i} H_{i}^{-1} A_{i}^{T}\right)^{-1} \cdot\left(B_{i} \delta \theta-A_{i} H_{i}^{-1} q_{i}+G_{i}\right)
$$


Now substituting equation (2-41) into equation (2-38):

$$
\boldsymbol{B}_{i}^{T} \cdot\left(\boldsymbol{A}_{i} \boldsymbol{H}_{i}^{-1} \boldsymbol{A}_{i}\right)^{-1} \cdot\left(\boldsymbol{B}_{i} \delta \theta-\boldsymbol{A}_{i} \boldsymbol{H}_{i}^{-1} \boldsymbol{q}_{i}+G_{i}\right)=0
$$

Solving for $\delta \theta$ we are left with:

$$
\delta \theta=\left(B_{i}^{T} \cdot\left(A_{i} H_{i}^{-1} A_{i}^{T}\right)^{-1} \cdot B_{i}\right)^{-1} \cdot B_{i}^{T} \cdot\left(A_{i} H_{i}^{-1} A_{i}^{T}\right)^{-1} \cdot\left(A_{i} H_{i}^{-1} q_{i}-G_{i}\right)
$$

We can define a new term $\mathrm{C}_{\mathrm{i}}$ as:

$$
C_{i} \equiv A_{i} H_{i}^{-1} A_{i}^{T}
$$

and a term $\mathrm{D}_{\mathrm{i}}$ as:

$$
D_{i} \equiv B_{i}^{T} C_{i}^{-1} B_{i}
$$

Substituting equations (2-44) and (2-45) into equation (2-43) gives:

$$
\delta \theta=D_{i}^{T} B_{i}^{T} C_{i}^{-1} \cdot\left(A_{i} H_{i}^{-1} q_{i}-G_{i}\right)
$$

Once we have calculated $\delta \theta$, we use equation (2-41) to calculate the Lagrangian multipliers which in turn are substituted into equation (2-39) to calculate the updates to the true measurements. Equations (2-39) and (2-46) then allow us to iteratively calculate the estimated beacon location and the estimated true values of the measurements with the recursion relations:

$$
\begin{aligned}
& \theta^{*}{ }_{i+1}=\theta^{*}{ }_{i}+\delta \theta \\
& \hat{W}_{i+1}^{*}=\hat{W}^{*}{ }_{i}+\delta \hat{W}
\end{aligned}
$$


All we need to start the calculations are initial values for $\theta^{*}$ and for $\mathbf{W}^{*}$. Since the measurement errors are presumably small, we can use the actual measured values $\mathbf{W}$ for the initial values of $\mathbf{W}^{*}$. However, since we have no idea of the location of the acoustic beacon, the choice of an initial value for $\theta^{*}$ is more difficult. In the absence of any other information, we can choose the center of the search area for the initial value of $\theta^{*}$.

In developing the solution to the maximum likelihood estimate we made the assumption that the measurement errors are normally distributed and independent between experiments. To determine if this is valid we refer back to the source of the measurement errors. The measurement error $\mathfrak{t}_{s \mu}$ is not normally distributed. However, if we do not make the assumption of a normally distributed error, and stay with a uniform distribution of errors, we will be unable to use the maximum likelihood method. This is because likelihood methods use gradient techniques to maximize a likelihood function which is based upon the probability distribution function of the errors. If the underlying probability distribution function is not smooth and continuous, there will not be a unique maximum, and a useful estimate can not be found. This means if we stay with the uniform distribution we will be forced to use a more computationally expensive direct search of the parameter space, or minimax method to estimate the acoustic beacon's location.

As a result of the uniformly distributed errors in $t_{s \mu}$, the solution that we obtain from the maximum likelihood estimator is suboptimal. There is also a chance that the method used to find the estimate that maximizes the likeliness function will diverge. 
This happens when the estimate of the true value of $t_{s \mu}$ is further away from the actual true value of $t_{s \mu}$ then the quantization of the time base allows. To prevent the solution from diverging, we can place additional constraints upon the minimization of the objective function that confine the solution to be within a feasible region. We define the feasible region to be the region bounded by the inequality constraints:

$$
\begin{aligned}
& - \text { Range }_{\max } \leq x_{T} \leq \text { Range }_{\max } \\
& - \text { Range }_{\max } \leq y_{T} \leq \text { Range }_{\max } \\
& - \text { Depth }_{\max } \leq z_{T} \leq 0
\end{aligned}
$$

Where Range max $_{\max }$ defines the size of the search area that we are willing to scan, and Depth $_{\max }$ is the maximum depth in the area of the search. The beacon's depth is bounded above by the sea surface level. If the solution to the maximum likelihood estimator is within the feasible region, we accept the estimate. If, however, the solution to the maximum likelihood estimator is outside the feasible region we must adjust the estimator to bring the solution inside the feasible region, or in the worst case, disregard the estimate.

To maintain a feasible solution we break equation (2-47) into component parts:

$$
\begin{aligned}
\theta^{*}{ }_{i+1} & =\theta^{*}{ }_{i}+\delta \theta=\theta^{*}{ }_{i}+\rho_{\theta} U_{\theta} \\
\hat{W}^{*}{ }_{i+1} & =\hat{W}^{*}{ }_{i}+\delta \hat{W}=\hat{W}^{*}{ }_{i}+\rho_{\hat{W}} U_{\hat{W}}
\end{aligned}
$$

where $U_{\theta}$ and $U_{w}^{\wedge}$ are unit magnitude vectors that describe the direction of $\delta \theta$ and $\delta \hat{W}$, and $\rho_{\theta}$ and $\rho_{\hat{W}}$ are the magnitudes of $\delta \theta$ and $\delta \hat{W}$. We assume that $\delta \theta$, and hence $U_{\theta}$, points in the proper direction to the maximum likelihood estimator. If 
$\theta_{i}^{*}+\delta \theta$ is outside the feasible region defined by equation (2-48), we find the maximum value of $\rho$ which satisfies:

$$
\left[\begin{array}{l}
\text { - }^{\text {Rang }} e_{\max } \\
- \text { Range }_{\max } \\
\text { Depth }_{\max }
\end{array}\right]<\theta_{i}^{*}+\rho_{\max } U_{\theta}<\left[\begin{array}{c}
\text { Range }_{\max } \\
\text { Range }_{\max } \\
0
\end{array}\right]
$$

Once we have found $\rho_{\max }$ we replace $\rho_{\theta}$ and $\rho_{\mathrm{w}}$ in equation (2-49) which gives the new update equations:

$$
\begin{gathered}
\theta^{*}{ }_{i+1}=\theta^{*}{ }_{i}+\frac{\rho_{\max }}{\sqrt{2}} U_{\theta} \\
\hat{W}^{*}{ }_{i+1}=\hat{W}^{*}{ }_{i}+\frac{\rho_{\max }}{\sqrt{2}} U_{\hat{W}}
\end{gathered}
$$

This essentially shortens the step that we move in updating $\theta^{*}$ and $\mathbf{W}^{*}$ for the iteration. Notice that we do not simply replace $\rho_{\theta}$ and $\rho_{\mathrm{W}}$ with $\rho_{\max }$. If we did this, $\theta_{\mathrm{i}+1}^{*}$ would be on the edge of the feasible region. With $\theta_{i+1}^{*}$ on the edge of the feasible region, $\rho_{\max }$ will be zero, and $\theta_{i+1}^{*}$ will equal $\theta_{\mathrm{i}}^{*}$ for all subsequent iterations. If this happens we will be unable to achieve any further updates of $\theta_{\mathrm{i}+1}^{*}$ and $\mathbf{W}_{\mathrm{i}}^{*}$. It is still possible that, after a certain number of iterations, $\theta_{i+1}^{*}$ lies on the edge of the feasible region. If this happens we must reject the estimate and try again using a different initial value for $\theta^{*}$ or more experimental data points.

\subsection{Algorithm}

Figure 2-4 at the end of this section describes the algorithm used to estimate the location of the acoustic beacon based on Deming's method as described by Bard. 
There is no natural way of telling whether or not progress towards the solution has occurred in any given iteration when we use this method [Bard 1974]. Bard suggests calculating the quantities:

$$
Z_{o}=\sum_{\mu=1}^{n} g_{\mu}\left(\hat{w}_{\mu_{i}}^{*}, \theta_{i}^{*}\right)^{T} c_{\mu_{i}}^{-1} g_{\mu}\left(\hat{w}_{\mu_{i}}^{*}, \theta_{i}^{*}\right)
$$

and:

$$
Z_{1}=\sum_{\mu=1}^{n} g_{\mu}\left(\hat{w}_{\mu_{i}}{ }^{*}+\delta \hat{w}_{\mu}, \theta_{i}^{*}+\delta \theta\right)^{T} c_{\mu_{i}}^{-1} g_{\mu}\left(\hat{w}_{\mu_{i}}{ }^{*}+\delta \hat{w}_{\mu}, \theta_{i}^{*}+\delta \theta\right)
$$

The rational behind these quantities follows that at the true values of $\mathbf{W}$ and $\theta$, $G(W, \theta)$ must equal zero. If $Q_{i}$ is a positive definite matrix, we require that:

$$
G\left(\hat{W}_{i+1}^{*}, \theta_{i+1}^{*}\right)^{T} Q_{i} G\left(\hat{W}_{i+1}^{*}, \theta_{i+1}^{*}\right) \leq G\left(\hat{W}_{i}^{*}, \theta_{i}^{*}\right)^{T} Q_{i} G\left(\hat{W}_{i}^{*}, \theta_{i}^{*}\right)
$$

This means that as $\mathbf{W}_{i}^{*}$ and $\theta_{i}^{*}$ approach the true values of $\mathbf{W}$ and $\theta$, the quantity $G\left(\mathbf{W}_{i}^{*}, \theta_{i}^{*}\right)^{T} Q_{i} G\left(W_{i}^{*}, \theta_{i}^{*}\right)$ must decrease. The natural choice for $Q_{i}$ is the inverse of the covariance matrix of $G\left(\mathbf{W}_{\mathrm{i}}^{*}, \theta_{\mathrm{i}}^{*}\right)$ [Bard 1974]. With $\mathrm{V}_{\mu}$ as the covariance matrix of the measurements, the covariance matrix of $\mathrm{G}\left(\mathbf{W}_{\mathrm{i}}^{*}, \theta_{\mathrm{i}}^{*}\right)$ is approximately:

$$
\operatorname{COV}\left(G\left(\hat{W}_{i}^{*}, \theta_{i}^{*}\right)\right) \approx \sum_{\mu=1}^{n} a_{\mu_{i}} V_{\mu} a_{\mu_{i}}^{T}
$$

Now from equation (2-30) we have:

$$
\boldsymbol{H}_{i} \equiv \partial^{2} \Phi\left(\hat{W}^{*}\right) / \partial \hat{W} \partial \hat{W}=\left[\begin{array}{cccc}
V_{1}^{-1} & 0 & \ldots & 0 \\
0 & V_{2}^{-1} & & \\
\vdots & & \\
0 & & \ldots & V_{n}^{-1}
\end{array}\right]
$$


So we choose:

$$
Q_{i}=\operatorname{COV}\left(G\left(\hat{W}_{i}^{*}, \theta_{i}^{*}\right)\right)^{-1}=\left(\sum_{\mu=1}^{n} a_{\mu_{i}} V_{\mu} a_{\mu_{i}}\right)^{-1}=\left(A_{i} H_{i}^{-1} A_{i}^{T}\right)^{-1}=C_{i}^{-1}
$$

It then follows that: [Bard 1974]

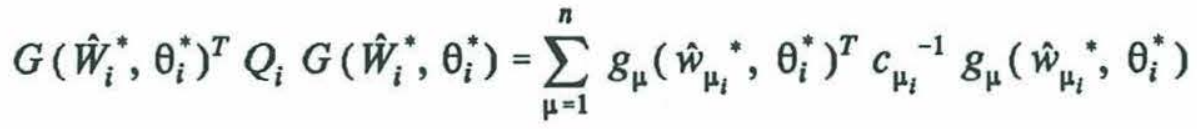

If $Z_{1}$ is less then $Z_{0}$, we accept $\delta \theta$ and $\delta W$ and continue to the next iteration. If $Z_{1}$ is greater then $Z_{0}$, we shorten $\delta \theta$ and $\delta \mathbf{W}$ and then recalculate $Z_{1}$.

To determine when to stop the algorithm we follow the termination criterion of Marquardt [Bard 1974]. We define a tolerance:

$$
\epsilon_{i}=10^{-4} \cdot\left(\left|\theta_{i}^{*}\right|_{\min }+10^{-3}\right)
$$

where $\left|\theta_{\mathrm{i}}^{*}\right|_{\min }$ is the smallest component of $\theta_{\mathrm{i}}^{*}$. The term $10^{-3}$ is added in case $\left|\theta_{i}^{*}\right|_{\min }$ is close to zero. For each iteration we check to see if $|\delta \theta|_{\min }$ is less then $\varepsilon_{\mathrm{i}}$. If $|\delta \theta|_{\min }$ is less then $\varepsilon_{\mathrm{i}}$, we terminate the algorithm. If $|\delta \theta|_{\min }$ is not less then $\varepsilon_{\mathrm{i}}$, we continue. For each iteration we calculate a new tolerance value $\varepsilon_{\mathrm{i}}$. This leads to a conservative termination criterion that will stop the algorithm if the parameter values cease changing.

To start the algorithm we choose an initial guess of the location of the 
acoustic beacon, $\theta_{0}^{*}$. We then use the measured values of $\mathbf{W}$ for the initial values of $\mathbf{W}_{\mathrm{o}}^{*}$. For each experiment $\mu$, we calculate:

$$
\begin{aligned}
& e_{\mu} \equiv \hat{w}_{\mu}{ }^{*}-w_{\mu} \\
& a_{\mu}=\partial g_{\mu} / \partial \hat{w} \\
& b_{\mu}=\partial g_{\mu} / \partial \theta
\end{aligned}
$$

For the case of the stationary beacon $a_{\mu}$ is given by:

$$
a_{\mu}=\left[\begin{array}{c}
\frac{\left(\hat{x}_{1}^{*}-x_{T}^{*}\right)}{\sqrt{\left(\hat{x}_{1}^{*}-x_{T}^{*}\right)^{2}+\left(\hat{y}_{1}^{*}-y_{T}^{*}\right)^{2}+\left(z_{T}^{*}\right)^{2}}} \\
\frac{\left(\hat{y}_{1}^{*}-y_{T}^{*}\right)}{\sqrt{\left(\hat{x}_{1}^{*}-x_{T}^{*}\right)^{2}+\left(\hat{y}_{1}^{*}-y_{T}^{*}\right)^{2}+\left(z_{T}^{*}\right)^{2}}} \\
-C \\
\frac{\left(\hat{x}_{2}^{*}-x_{T}^{*}\right)}{\sqrt{\left(\hat{x}_{2}^{*}-x_{T}^{*}\right)^{2}+\left(\hat{y}_{2}^{*}-y_{T}^{*}\right)^{2}+\left(z_{T}^{*}\right)^{2}}} \\
-\frac{\left(\hat{y}_{2}^{*}-y_{T}^{*}\right)}{\sqrt{\left(\hat{x}_{2}^{*}-x_{T}^{*}\right)^{2}+\left(\hat{y}_{2}^{*}-y_{T}^{*}\right)^{2}+\left(z_{T}^{*}\right)^{2}}} \\
+C
\end{array}\right]
$$

and $b_{\mu}$ is given by: 


$$
b_{\mu}=\left[\begin{array}{c}
\frac{\left(\hat{x}_{2}^{*}-x_{T}^{*}\right)}{\sqrt{\left(\hat{x}_{2}^{*}-x_{T}^{*}\right)^{2}+\left(\hat{y}_{2}^{*}-y_{T}^{*}\right)^{2}+\left(z_{T}^{*}\right)^{2}}}-\frac{\left(\hat{x}_{1}^{*}-x_{T}^{*}\right)}{\sqrt{\left(\hat{x}_{1}^{*}-x_{T}^{*}\right)^{2}+\left(\hat{y}_{1}^{*}-y_{T}^{*}\right)^{2}+\left(z_{T}^{*}\right)^{2}}} \\
\frac{\left(\hat{y}_{2}^{*}-y_{T}^{*}\right)}{\sqrt{\left(\hat{x}_{2}^{*}-x_{T}^{*}\right)^{2}+\left(\hat{y}_{2}^{*}-y_{T}^{*}\right)^{2}+\left(z_{T}^{*}\right)^{2}}}-\frac{\left(\hat{y}_{1}^{*}-y_{T}^{*}\right)}{\sqrt{\left(\hat{x}_{1}^{*}-x_{T}^{*}\right)^{2}+\left(\hat{y}_{1}^{*}-y_{T}^{*}\right)^{2}+\left(z_{T}^{*}\right)^{2}}} \\
\frac{z_{T}^{*}}{\sqrt{\left(\hat{x}_{1}^{*}-x_{T}^{*}\right)^{2}+\left(\hat{y}_{1}^{*}-y_{T}^{*}\right)^{2}+\left(z_{T}^{*}\right)^{2}}}-\frac{z_{T}^{*}}{\sqrt{\left(\hat{x}_{2}^{*}-x_{T}^{*}\right)^{2}+\left(\hat{y}_{2}^{*}-y_{T}^{*}\right)^{2}+\left(z_{T}^{*}\right)^{2}}}
\end{array}\right]
$$

Once we have $a_{\mu}$ and $b_{\mu}$, we calculate:

$$
\begin{gathered}
c_{\mu}=a_{\mu}{ }^{T} V_{\mu} a_{\mu} \\
Z_{0}=\sum_{\mu=1}^{n} \frac{g\left(\hat{w}_{\mu}{ }^{*}, \theta^{*}\right)^{2}}{c_{\mu}}
\end{gathered}
$$

and:

$$
D=\sum_{\mu=1}^{n}\left(\frac{1}{c_{\mu}}\right) \cdot b_{\mu} b_{\mu}{ }^{T}
$$

Using equation (2-46) we can solve for $\delta \theta$ :

$$
\delta \theta=D^{-1} \cdot \sum_{\mu=1}^{n}\left(\frac{1}{c_{\mu}}\right) \cdot\left(a_{\mu}{ }^{T} e_{\mu}-g\left(\hat{w}_{\mu}{ }^{*}, \theta^{*}\right)\right) \cdot b_{\mu}
$$

Now using equation (2-41) we calculate the Lagrangian multipliers:

$$
\lambda_{\mu}=\left(\frac{1}{c_{\mu}}\right) \cdot\left(b_{\mu}{ }^{T} \delta \theta-a_{\mu}{ }^{T} e_{\mu}+g\left(\hat{w}_{\mu}{ }^{*}, \theta^{*}\right)\right)
$$


and finally we calculate:

$$
\delta \hat{w}_{\mu}=-e_{\mu}-\lambda_{\mu} V_{\mu} a_{\mu}
$$

Once we have $\delta \theta$ and $\delta \mathbf{W}$ we check to see if $\theta_{i}^{*}+\delta \theta$ is in the feasible region. If $\theta_{i}^{*}+\delta \theta$ is not in the feasible region, we adjust the step size of $\delta \theta$ as describe earlier. Once we have a feasible step for $\delta \theta$, we calculate:

$$
Z_{1}=\sum_{\mu=1}^{n} \frac{g\left(\hat{w}_{\mu}^{*}+\delta \hat{w}_{\mu}, \theta^{*}+\delta \theta\right)^{2}}{c_{\mu}}
$$

If $Z_{1}$ is less then $Z_{0}$, we accept the values of $\delta \theta$ and $\delta \mathbf{W}$ and update $\theta^{*}$ and $\mathbf{W}^{*}$ using equation (2-47). We then move to the next iteration. If $Z_{1}$ is greater then $Z_{0}$, we assume that we have overshot the maximum likelihood estimate, shorten $\delta \theta$ and $\delta \mathbf{W}$ by a factor of two, then recalculate $Z_{1}$. We continue this process until $Z_{1}$ is less then $Z_{0}$, or until $\delta \theta$ is negligible, at which point we have to stop the algorithm and begin again with a new initial value for $\theta^{*}$, or more experimental data points. 


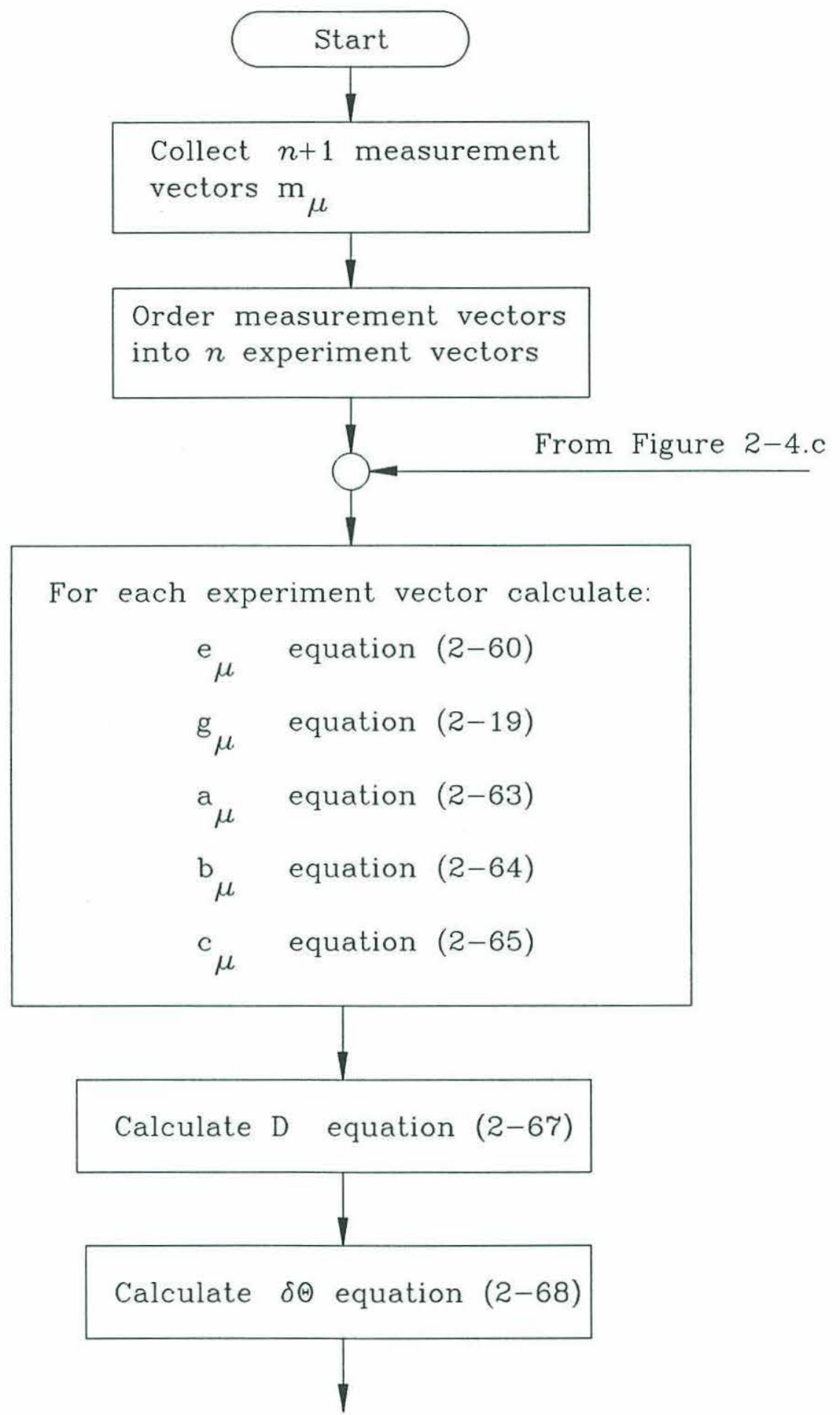

To Figure 2-4.b

Figure 2-4.a 


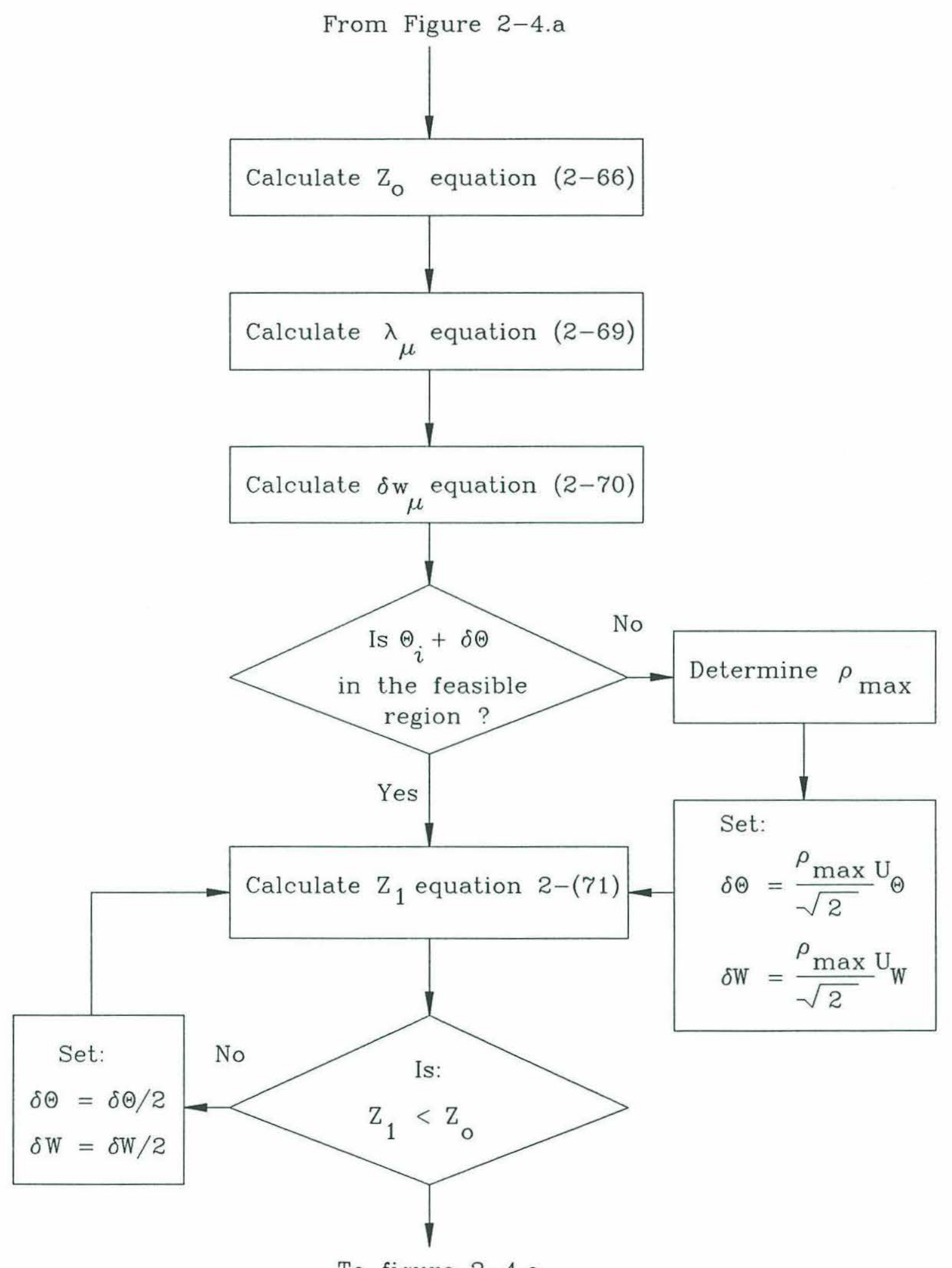

To figure 2-4.c

Figure 2-4.b 
From Figure 2-4.b

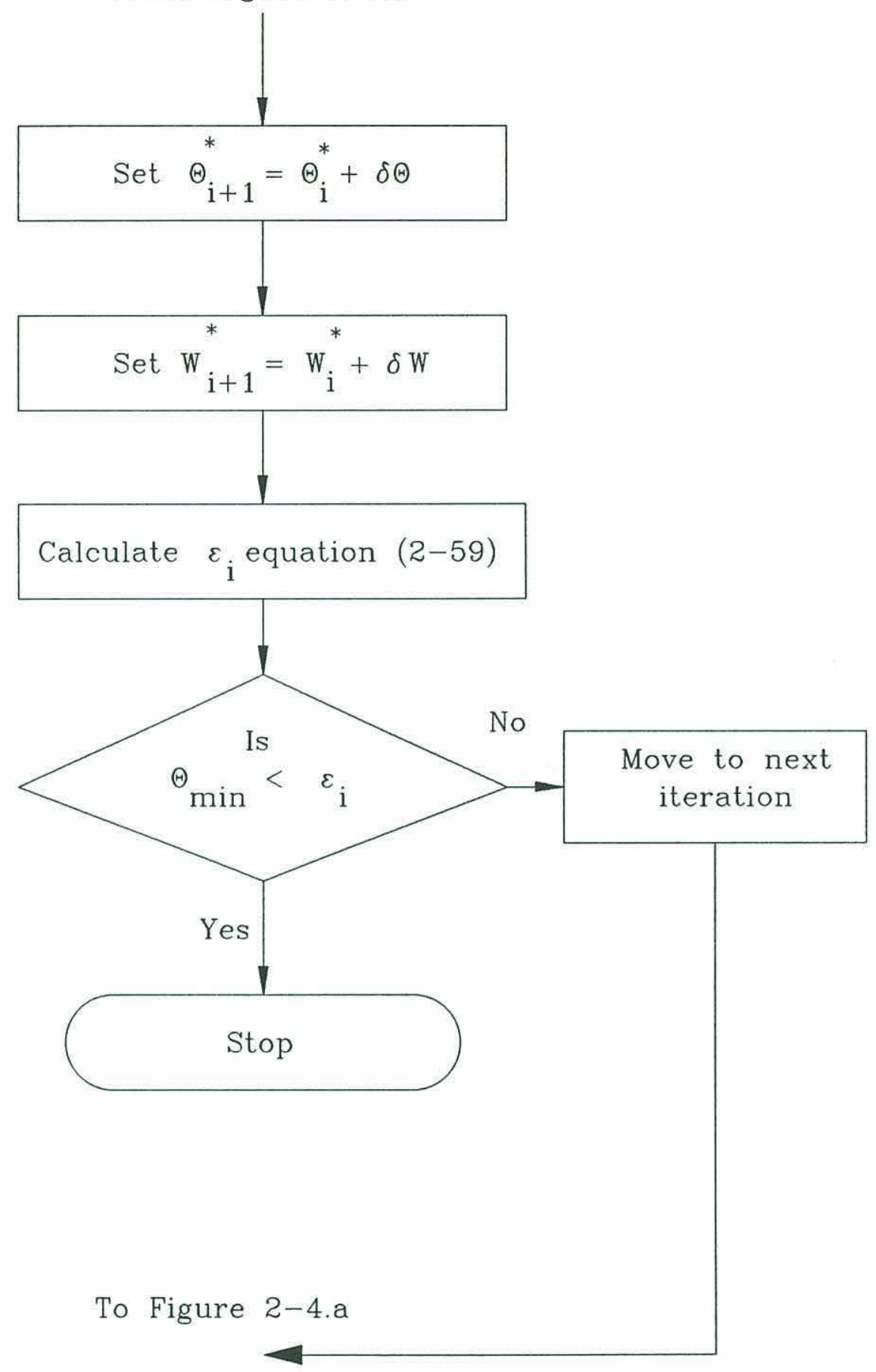

Figure 2-4.c 


\subsection{Accuracy of the Estimation}

One of the benefits of using Deming's method for the maximum likelihood estimate is that the covariance of the estimated parameters is approximated by the inverse of the matrix D defined by equation (2-45) [Bard 1974]. This lets us quickly determine if the estimation of the acoustic beacon's position is acceptable, or if more experimental data needs to be collected to improve the estimation. We are also able to test if the assumptions made regarding the covariance matrix of the measurement errors are valid by examining the final residual vectors. We define a moment matrix $\mathrm{M}$ as:

$$
M=\sum_{\mu=1}^{n} e_{\mu}^{*} e_{\mu}^{* T}
$$

where the $e_{\mu}^{*}$ 's are the final residual vectors. To estimate the covariance of the residuals we take:

$$
\tilde{V}=\frac{6}{n-3} \cdot M
$$

where $n$ is the number of experiment vectors used in the maximum likelihood estimation. Because of the nature of the constrained minimization problem, the first term in equation (2-73) is needed to correct for bias [Bard 1974]. We can compare the outcome of equation (2-73) to the assumed covariance matrix $V_{\mu}$ defined by equation (2-17) to see if the estimated covariance matrix $\mathrm{V}_{\mu}$ is valid. 


\subsection{Summary}

In this chapter we have developed the maximum likelihood estimate of the acoustic beacon's position, and a means in which to gauge the accuracy of the estimation. To estimate the beacon's position we defined a structural model that requires the observer to measure the time of receipt and location of receipt of two different signal from the acoustic beacon. The choice of how far apart the two signals that define an experiment vector $\left(\mathrm{w}_{\mu}\right)$ must be, and the number of experiment vectors to use in the parameter estimation problem $(n)$ remain as free parameters. In chapter IV we examine the performance of the algorithm of this chapter for different choices of these two free parameters. 


\section{Chapter III}

\section{Moving Acoustic Beacon}

\subsection{Introduction}

In chapter II we developed the maximum likelihood estimator for a stationary acoustic beacon. In this chapter we extend the solution to include a moving acoustic beacon. The task of localizing an arbitrarily moving acoustic beacon is quite difficult. Abrupt changes in the acoustic beacon's velocity are difficult to parameterize and model. Therefore, we concentrate on the case of an acoustic beacon which moves in a constant velocity, linear path. The assumption of constant velocity, linear motion is a reasonable assumption for acoustic beacons located on instruments ascending at terminal velocity from the bottom, or for acoustic beacons located on instruments moving with an ocean current that is steady in the area of interest. With the assumption of constant linear motion, we modify the structural model given by equation (2-19) to include parameters that reflect the acoustic beacon's velocity. Once the new structural model is defined, we show that the solution to the maximum likelihood estimate for the moving beacon follows directly from the solution to the maximum likelihood estimate for the stationary acoustic beacon. Next, the algorithm developed to solve the stationary acoustic beacon localization problem is extended using the new structural model so that the position and velocity parameters of the moving acoustic beacon can be determined. Finally, the accuracy of the estimator is examined. 


\subsection{Problem Geometry}

Consider an acoustic beacon having constant velocity components given by $\mathrm{v}_{\mathrm{x}}, \mathrm{v}_{\mathrm{y}}$, and $\mathrm{v}_{\mathrm{z}}$. We define a new parameter vector:

$$
\theta=\left[\begin{array}{llllll}
x_{0} & y_{0} & z_{0} & v_{x} & v_{y} & v_{z}
\end{array}\right]^{T}
$$

where the coordinates $x_{0}, y_{0}$, and $z_{0}$ define the acoustic beacon's initial position on the same Cartesian coordinate system that was described in chapter II. For convenience, we assume the velocity components $v_{x}, v_{y}$, and $v_{z}$ have the units of meters per second. The position of the acoustic beacon at an arbitrary time $t_{\beta}$ can by found using:

$$
\left[\begin{array}{l}
x_{T} \\
y_{T} \\
z_{T}
\end{array}\right]=\left[\begin{array}{c}
x_{o} \\
y_{o} \\
z_{o}
\end{array}\right]+\left(t_{\beta}-T_{o}\right)\left[\begin{array}{c}
v_{x} \\
v_{y} \\
v_{z}
\end{array}\right]
$$

where $T_{0}$ is the reference time that corresponds to the acoustic beacon's initial position $x_{0}, y_{0}, z_{0}$

As in chapter II, we define a measurement vector $\mathrm{m}_{\mu}$ as:

$$
m_{\mu}=\left[\begin{array}{lll}
x_{\mu} & y_{\mu} & t_{s \mu}
\end{array}\right]^{T}
$$

Where $x_{\mu}$ and $y_{\mu}$ are the ship's coordinates, in meters, on the same local Cartesian coordinate system defined for the acoustic beacon, and $t_{s \mu}$ is the synchronized time of receipt that corresponds to the observer's position $x_{\mu}, y_{\mu}$. 


\subsection{Modified Structural Model}

To account for the linearly moving acoustic beacon, we redefine the function $\mathbf{f}\left(\mathbf{m}_{\mu}, \theta\right)$ from chapter II as:

$$
\begin{aligned}
f\left(m_{\mu}, \theta\right) & \equiv \sqrt{\left[x_{\mu}-x_{o}-\left(t_{\beta}-T_{o}\right) v_{x}\right]^{2}+\left[y_{\mu}-y_{o}-\left(t_{\beta}-T_{o}\right) v_{y}\right]^{2}+\left[z_{o}+\left(t_{\beta}-T_{o}\right) v_{z}\right]^{2}} \\
& -\tau\left(C, t_{s \mu}\right)
\end{aligned}
$$

where $t_{\beta}$ represents the time that the acoustic beacon transmitted the signal that the observer received at the synchronized time of receipt $t_{s \mu}$. Using the same homogeneous ocean model, and direct path propagation from chapter II, we simplify equation (3-4) to:

$$
\begin{aligned}
f\left(m_{\mu}, \theta\right) & \equiv \sqrt{\left[x_{\mu}-x_{o}-\left(t_{\beta}-T_{o}\right) v_{x}\right]^{2}+\left[y_{\mu}-y_{o}-\left(t_{\beta}-T_{o}\right) v_{y}\right]^{2}+\left[z_{o}+\left(t_{\beta}-T_{o}\right) v_{z}\right]^{2}} \\
& -C\left(t_{s \mu}-T_{o}\right)
\end{aligned}
$$

Now assume that at time $T_{0}$ the acoustic beacon transmitted the first signal that the observer receives. To find when the current signal received at the synchronized time of receipt $t_{s \mu}$ was transmitted, we note that the acoustic beacon transmits a signal for every pulse repetition interval. With $T_{0}$ as a reference, $t_{\beta}$ can be found by:

$$
t_{\beta}=T_{o}+i \cdot T_{p}
$$

where $\mathrm{T}_{\mathrm{p}}$ is the pulse repetition interval of the acoustic beacon and $i$ is number of pulse repetition intervals that separate the signal transmitted at time $t_{\beta}$ from the signal transmitted at reference time $\mathrm{T}_{\mathrm{o}}$. To determine $i$, the observer counts how many signals are received between the first received signal and the signal received at the 
synchronized time of receipt $t_{s \mu}$. Simplifying equation (3-5) to eliminate $T_{o}$ and $t_{\beta}$, we substitute equation (3-6) into equation (3-5) which yields:

$$
\begin{aligned}
f\left(m_{\mu}, \theta\right)= & \sqrt{\left(x_{\mu}-x_{o}-i \cdot T_{p} \cdot v_{x}\right)^{2}+\left(y_{\mu}-y_{o}-i \cdot T_{p} \cdot v_{y}\right)^{2}+\left(z_{o}+i \cdot T_{p} \cdot v_{z}\right)^{2}} \\
& -C \cdot\left(t_{s \mu}-T_{o}\right)
\end{aligned}
$$

Now, just as in the case of the stationary acoustic beacon, we must eliminate the dependence on the time reference $T_{0}$ from the last term in equation (3-7).

To eliminate the dependence upon the time reference $T_{0}$, we define the function $\mathbf{g}\left(\mathbf{m}_{1}, \mathbf{m}_{2}, \theta\right)$ as:

$$
g\left(m_{1}, m_{2}, \theta\right) \equiv f\left(m_{1}, \theta\right)-f\left(m_{2}, \theta\right)
$$

Substituting equation (3-7) into equation (3-8) with $\mathrm{m}_{1}$ for the first received signal and $\mathrm{m}_{2}$ for the second received signal we have:

$$
\begin{aligned}
g\left(m_{1}, m_{2}, \theta\right)= & \sqrt{\left(x_{1}-x_{o}-i \cdot T_{p} \cdot v_{x}\right)^{2}+\left(y_{1}-y_{o}-i \cdot T_{p} \cdot v_{y}\right)^{2}+\left(z_{o}+i \cdot T_{p} \cdot v_{z}\right)^{2}} \\
& -\sqrt{\left(x_{2}-x_{o}-j \cdot T_{p} \cdot v_{x}\right)^{2}+\left(y_{2}-y_{o}-j \cdot T_{p} \cdot v_{y}\right)^{2}+\left(z_{o}+j \cdot T_{p} \cdot v_{z}\right)^{2}} \\
& -C \cdot\left(t_{s 1}-t_{s 2}\right)
\end{aligned}
$$

Simplifying notation as in equation (2-13), and showing the dependence upon the true value of the measurements, we define the structural model for the case of the moving acoustic beacon as: 


$$
\begin{aligned}
g\left(\hat{w}_{\mu}, \theta\right)= & \sqrt{\left(\hat{x}_{1}-x_{o}-i \cdot T_{p} \cdot v_{x}\right)^{2}+\left(\hat{y}_{1}-y_{o}-i \cdot T_{p} \cdot v_{y}\right)^{2}+\left(z_{o}+i \cdot T_{p} \cdot v_{z}\right)^{2}} \\
& -\sqrt{\left(\hat{x}_{2}-x_{o}-j \cdot T_{p} \cdot v_{x}\right)^{2}+\left(\hat{y}_{2}-y_{o}-j \cdot T_{p} \cdot v_{y}\right)^{2}+\left(z_{o}+j \cdot T_{p} \cdot v_{z}\right)^{2}} \\
& -C \cdot\left(\hat{t}_{s 1}-\hat{t}_{s 2}\right)
\end{aligned}
$$

This is analogous to equation (2-19) with additional terms included to correct for the beacon's motion.

\subsection{Maximum Likelihood Estimator Solution}

The solution to the maximum likelihood estimation problem for the case of the moving acoustic beacon follows directly from the solution to the maximum likelihood estimation problem for the case of the stationary acoustic beacon. Since only the structural model has changed, the likelihood function $\log (\mathrm{L}(\mathbf{W}, \mathrm{V}))$, the Lagrangian function $\Lambda\left(\mathbf{W}, \theta, V, \lambda_{1}, \ldots, \lambda_{n}\right)$ and the objective function $\Phi\left(\mathbf{W}_{\mathrm{i}}^{*}\right)$ all have the same form as the corresponding functions defined for the stationary beacon. To solve for the maximum likelihood estimator of the parameter vector $\theta$, we simply substitute the modified structural model given by equation (3-10) for the stationary structural mode given by equation (2-19), and follow the method of Deming described in chapter II. There are, however, a few differences that must be accounted for.

If the measurement errors are the same for both cases, we still expect the solution to the maximum likelihood estimator to be suboptimal. Because of the uniform distribution of the measurement errors $\mathfrak{t}_{s \mu}$, we must continue to confine the solution of the maximum likelihood estimation problem to a feasible region. With the addition of the parameters $v_{x}, v_{y}$, and $v_{z}$ to $\theta$, we expand the feasible region defined by 
equation $(2-48)$ to:

$$
\begin{aligned}
- \text { Range }_{\max } & \leq x_{0} \leq \text { Range }_{\max } \\
- \text { Range }_{\max } & \leq y_{0} \leq \text { Range }_{\max } \\
- \text { Depth }_{\max } & \leq z_{0} \leq 0 \\
-v_{x_{\max }} & \leq v_{x} \leq v_{x_{\max }} \\
-v_{y_{\max }} & \leq v_{y} \leq v_{y_{\max }} \\
-v_{z_{\max }} & \leq v_{z} \leq v_{z_{\max }}
\end{aligned}
$$

As in chapter II, to maintain a feasible solution, we break $\delta \theta$ into component parts:

$$
\theta_{i+1}^{*}=\theta_{i}^{*}+\delta \theta=\theta_{i}^{*}+\rho_{\theta} U_{\theta}
$$

where $U_{\theta}$ is unit magnitude vector that describes the direction of $\delta \theta$, and $\rho_{\theta}$ is the magnitude of $\delta \theta$. We assume that $\delta \theta$, and therefore $\mathrm{U}_{\theta}$, points in the proper direction to the maximum likelihood estimator. If $\theta_{\mathrm{i}}^{*}+\delta \theta$ is outside the feasible region defined by equation (3-11), we find the maximum value of $\rho$ that satisfies:

$$
\left[\begin{array}{c}
- \text { Range }_{\max } \\
- \text { Range }_{\max } \\
- \text { Depth }_{\max } \\
-v_{x_{\max }} \\
-v_{y_{\max }} \\
-v_{z_{\max }}
\end{array}\right]<\theta_{i}^{*}+\rho_{\max } U_{\theta}<\left[\begin{array}{c}
\text { Range }_{\max } \\
\text { Range }_{\max } \\
0 \\
v_{x_{\max }} \\
v_{y_{\max }} \\
v_{z_{\max }}
\end{array}\right]
$$

Once we have found $\rho_{\max }$, we define the new recursion relations: 


$$
\begin{aligned}
& \theta_{i+1}^{*}=\theta_{i}^{*}+\frac{\rho_{\max }}{\sqrt{2}} U_{\theta} \\
& \hat{W}_{i+1}^{*}=\hat{W}_{i}^{*}+\frac{\rho_{\max }}{\sqrt{2}} U_{\hat{W}}
\end{aligned}
$$

As with the case of the stationary acoustic beacon, if, after a few iterations, $\theta_{i}^{*}$ lies on the edge of the feasible region, $\rho_{\max }$ will be zero and $\theta_{i+1}^{*}$ will equal $\theta_{i}^{*}$ for all subsequent iterations. If this happens we reject the estimate, and try again using a different initial value for $\theta^{*}$, or more experimental data points.

\subsection{Algorithm}

Figure 3-1 at the end of this section describes the algorithm used to solve for the acoustic beacon's position and velocity. The algorithm is essentially the same algorithm developed in chapter II with changes made to account for the modified structural model of equation (3-10). Because of the modified structural model, we must reevaluate the quantities $a_{\mu}$ and $b_{\mu}$. From equation (3-10), we define the quantities:

$$
\begin{gathered}
d_{1} \equiv \sqrt{\left(\hat{x}_{1}^{*}-x_{o}^{*}-i \cdot T_{p} v_{x}^{*}\right)^{2}+\left(\hat{y}_{1}^{*}-y_{o}^{*}-i \cdot T_{p} v_{y}^{*}\right)^{2}+\left(z_{o}^{*}+i \cdot T_{p} v_{z}^{*}\right)^{2}} \\
d_{2} \equiv \sqrt{\left(\hat{x}_{2}^{*}-x_{o}^{*}-j \cdot T_{p} \cdot v_{x}^{*}\right)^{2}+\left(\hat{y}_{2}^{*}-y_{o}^{*}-j \cdot T_{p} \cdot v_{y}^{*}\right)^{2}+\left(z_{o}^{*}+j \cdot T_{p} \cdot v_{z}^{*}\right)^{2}}
\end{gathered}
$$

$a_{\mu}$ and $b_{\mu}$ are then given by: 


$$
a_{\mu}=\partial g\left(\hat{w}_{\mu}{ }^{*}, \theta^{*}\right) / \partial \hat{w}_{\mu}=\left[\begin{array}{c}
\frac{\left(\hat{x}_{1}{ }^{*}-x_{o}^{*}-i \cdot T_{p} \cdot v_{x}{ }^{*}\right)}{d_{1}} \\
\frac{\left(\hat{y}_{1}{ }^{*}-y_{o}^{*}-i \cdot T_{p} \cdot v_{y}^{*}\right)}{d_{1}} \\
-C \\
\frac{\left(\hat{x}_{2}{ }^{*}-x_{o}^{*}-j \cdot T_{p} \cdot v_{x}^{*}\right)}{d_{2}} \\
\frac{\left(\hat{y}_{2}{ }^{*}-y_{o}^{*}-j \cdot T_{p} \cdot v_{y}^{*}\right)}{d_{2}} \\
C
\end{array}\right]
$$

$b_{\mu}=\partial g\left(\hat{w}_{\mu}{ }^{*}, \theta^{*}\right) / \partial \theta$

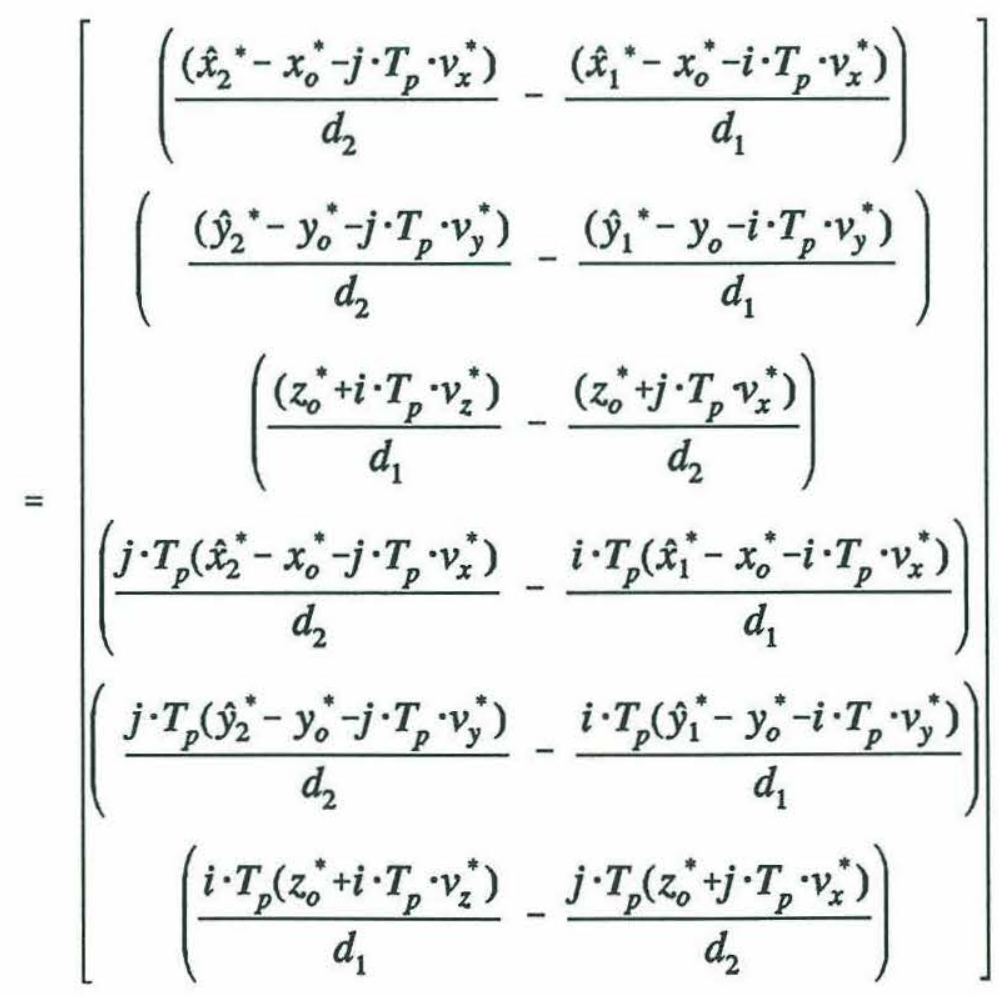


To evaluate the structural model, and the terms $a_{\mu}$ and $b_{\mu}$, we need to know the values of $i$ and $j$ that represent the time that the received signals were transmitted. This forces the observer to keep a count of the number of signals that are received, with the first received signal corresponding to $i=0$.

Once we have found the maximum likelihood estimate of the parameter vector $\theta$, we need to evaluate the estimated current position of the acoustic beacon. The position defined by $\mathrm{x}_{\mathrm{o}}{ }^{*}, \mathrm{y}_{\mathrm{o}}^{*}, \mathrm{z}_{\mathrm{o}}^{*}$ is the estimated initial position of the acoustic beacon at the time $\mathrm{T}_{\mathrm{o}}$. We assume that the time $\mathrm{T}_{\mathrm{o}}$ corresponds to the case $i=0$. To determine the current position of the acoustic beacon, we project the solution forward along the path described by the estimated velocity components $\mathrm{v}_{\mathrm{x}}^{*}, \mathrm{v}_{\mathrm{y}}^{*}, \mathrm{v}_{\mathrm{z}}^{*}$. The estimated current position of the acoustic beacon is then given by:

$$
\left[\begin{array}{c}
x_{T}^{*} \\
y_{T}^{*} \\
z_{T}^{*}
\end{array}\right]=\left[\begin{array}{c}
x_{o}^{*} \\
y_{o}^{*} \\
z_{o}^{*}
\end{array}\right]+i \cdot T_{p}\left[\begin{array}{c}
v_{x}^{*} \\
v_{y}^{*} \\
v_{z}^{*}
\end{array}\right]
$$

where $i$ is the total number of beacon signals received and $T_{p}$ is the pulse repetition interval of the acoustic beacon. The quantity $\left(i \cdot \mathrm{T}_{\mathrm{p}}\right)$ represents the time it took to collect the measurements used to calculate the maximum likelihood estimator. 


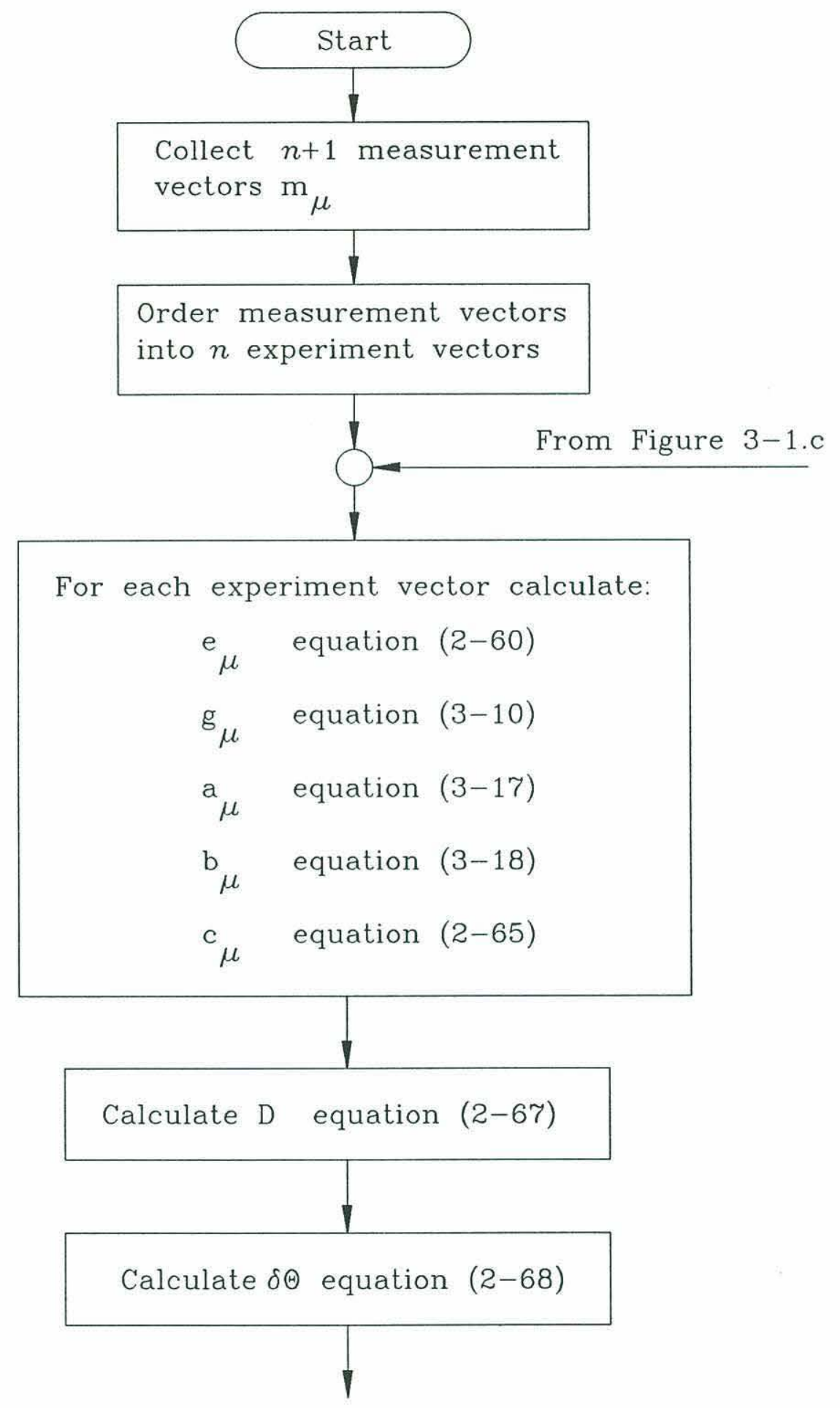

To Figure 3-1.b

Figure 3-1.a 


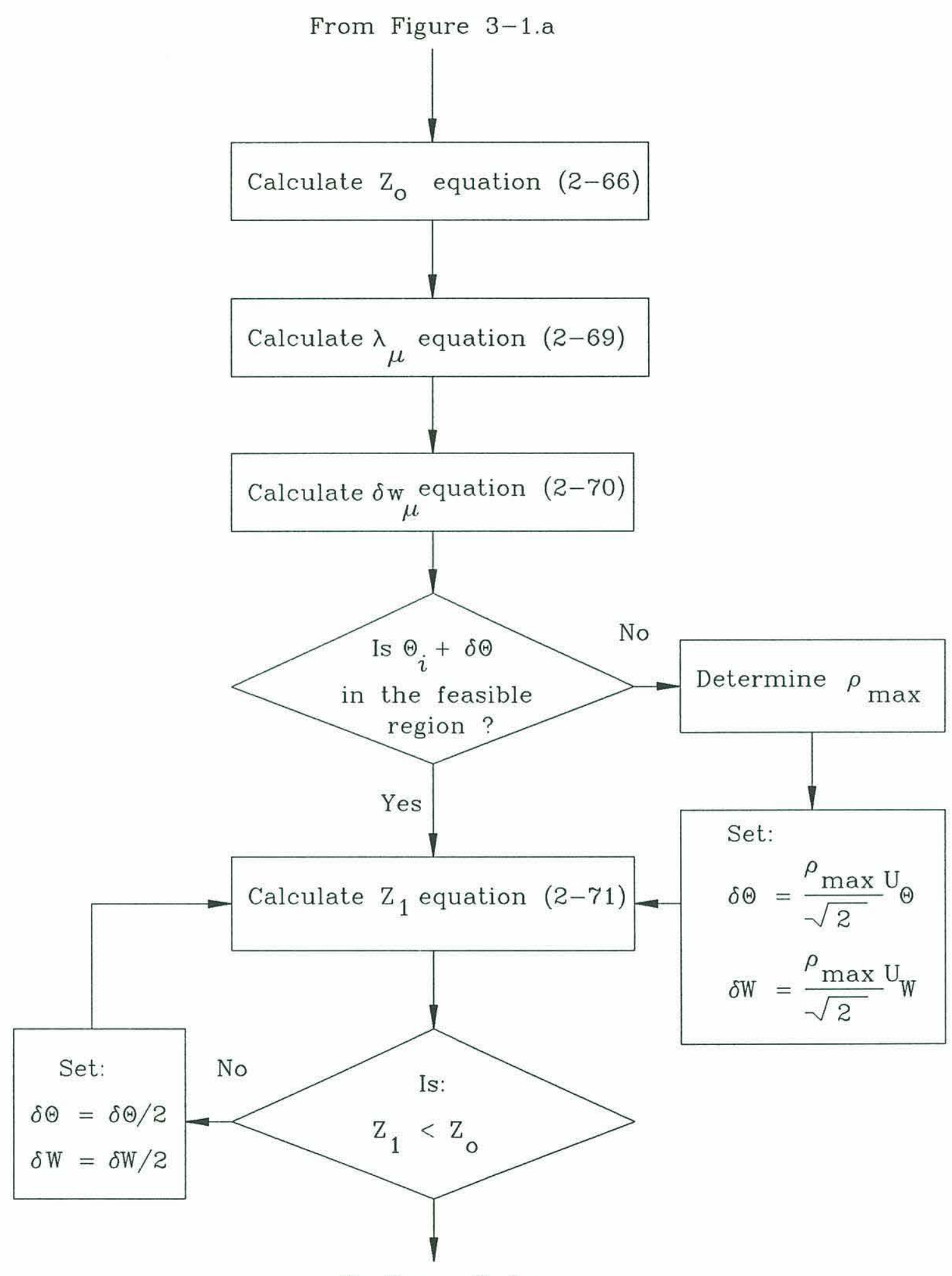

To figure 3-1.c

Figure $3-1 . \mathrm{b}$ 
From Figure 3-1.b

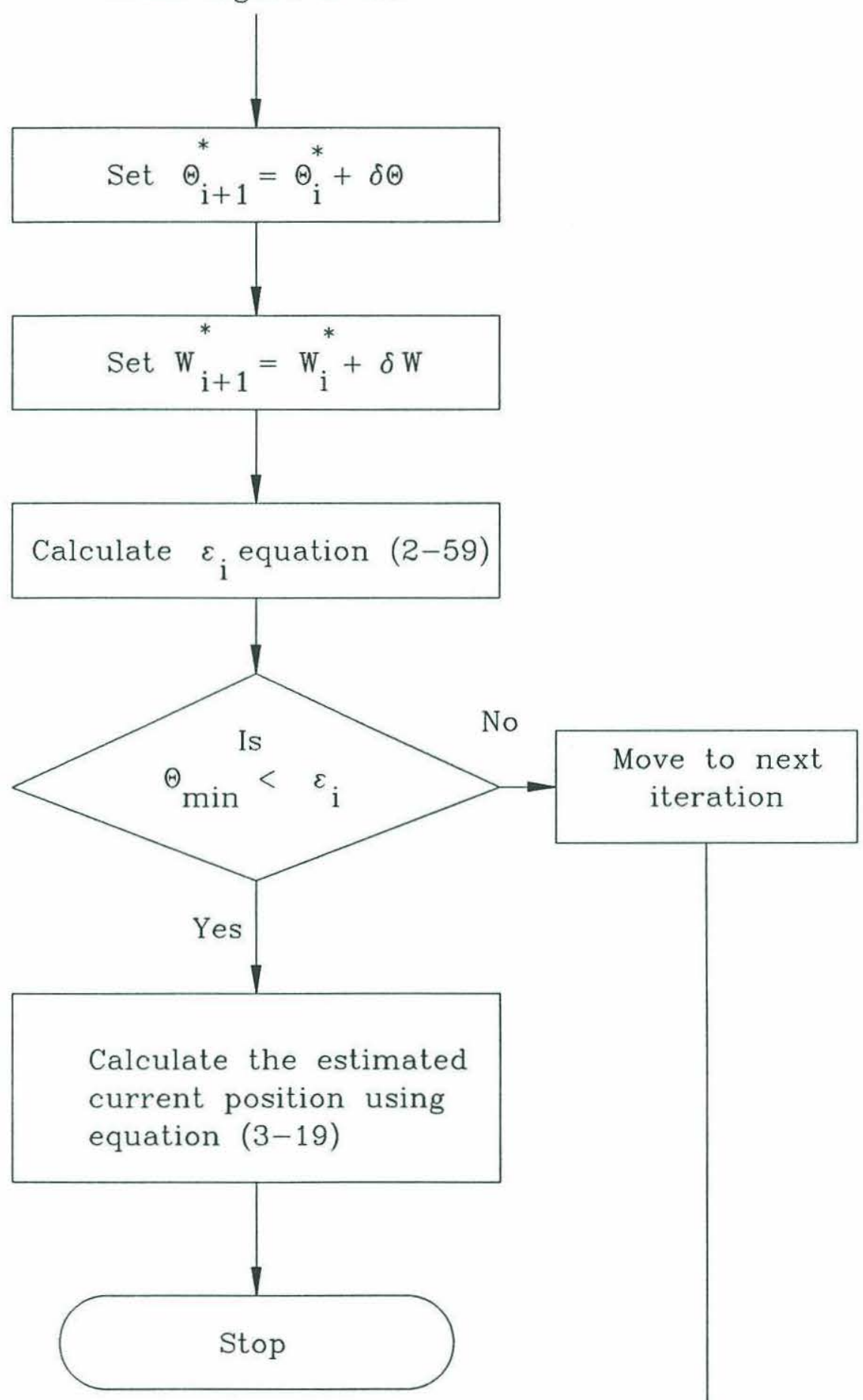

To Figure 3-1.a

Figure $3-1 . c$ 


\subsection{Accuracy of the Estimator}

To estimate the accuracy of the solution to the maximum likelihood estimator, we examine the estimated covariance matrix of the parameter vector $\theta$. As with the stationary beacon, the estimated covariance matrix of the parameter vector $\theta$ is given by the inverse of the matrix $\mathrm{D}$ or:

$$
V_{\theta}^{*}=\left(B^{T} C^{-1} B\right)^{-1}=\left(\sum_{\mu=1}^{n}\left(\frac{1}{c_{\mu}}\right) \cdot b_{\mu} b_{\mu}\right)^{-1}
$$

The variance of the velocity components $\mathrm{v}_{\mathrm{x}}{ }^{*}, \mathrm{v}_{\mathrm{y}}{ }^{*}$, and $\mathrm{v}_{\mathrm{z}}{ }^{*}$ are the last three diagonal elements of the covariance matrix $\mathrm{V}_{\theta}^{*}$.

To determine the variances of the current acoustic beacon position estimates we calculate:

$$
\begin{aligned}
& \sigma_{x_{T}}{ }^{2}=E\left[\left(\left(x_{o}+i \cdot T_{p} v_{x}\right)-E\left[x_{o}+i \cdot T_{p} v_{x}\right]\right)^{2}\right] \\
& \sigma_{y_{T}}{ }^{2}=E\left[\left(\left(y_{o}+i \cdot T_{p} v_{y}\right)-E\left[y_{o}+i \cdot T_{p} v_{y}\right]\right)^{2}\right] \\
& \sigma_{z_{T}}{ }^{2}=E\left[\left(\left(z_{o}+i \cdot T_{p} v_{z}\right)-E\left[z_{o}+i \cdot T_{p} v_{z}\right]\right)^{2}\right]
\end{aligned}
$$

Simplifying equations (3-21) through (3-23) yields:

$$
\begin{aligned}
& \sigma_{x_{T}}{ }^{2}= \sigma_{x_{o}}{ }^{2}+\left(i \cdot T_{p}\right)^{2} \cdot \sigma_{v_{x}}{ }^{2}+2 \cdot\left(i \cdot T_{p}\right) E\left[x_{o} v_{x}\right] \\
&-E\left[x_{o}\right]^{2}-2 \cdot\left(i \cdot T_{p}\right) E\left[v_{x}\right] E\left[x_{o}\right]-\left(i \cdot T_{p} \cdot E\left[v_{x}\right]\right)^{2} \\
& \sigma_{y_{T}}{ }^{2}= \sigma_{y_{o}}{ }^{2}+\left(i \cdot T_{p}\right)^{2} \cdot \sigma_{v_{y}}{ }^{2}+2 \cdot\left(i \cdot T_{p}\right) E\left[y_{o} v_{y}\right] \\
&-E\left[y_{o}\right]^{2}-2 \cdot\left(i \cdot T_{p}\right) E\left[v_{y}\right] E\left[y_{o}\right]-\left(i \cdot T_{p} \cdot E\left[v_{y}\right]\right)^{2} \\
& 56
\end{aligned}
$$




$$
\begin{aligned}
\sigma_{z_{T}}{ }^{2}= & \sigma_{z_{o}}{ }^{2}+\left(i \cdot T_{p}\right)^{2} \cdot \sigma_{v_{z}}{ }^{2}+2 \cdot\left(i \cdot T_{p}\right) E\left[z_{o} v_{z}\right] \\
& -E\left[z_{o}\right]^{2}-2 \cdot\left(i \cdot T_{p}\right) E\left[v_{z}\right] E\left[z_{o}\right]-\left(i \cdot T_{p} \cdot E\left[v_{z}\right]\right)^{2}
\end{aligned}
$$

Now if we assume that the estimate of the parameter vector $\theta$ is unbiased, we can substitute the estimated parameter values for their means in equations (3-24) through (3-26). This gives us a method in which to calculate the estimated variance of the projected current position:

$$
\begin{aligned}
\sigma_{x_{T}}{ }^{2}= & \sigma_{x_{o}}{ }^{2}+\left(i \cdot T_{p}\right)^{2} \cdot \sigma_{v_{x}}{ }^{2}+2 \cdot\left(i \cdot T_{p}\right) E\left[x_{o} v_{x}\right] \\
& -\left(x_{o}^{*}\right)^{2}-2 \cdot\left(i \cdot T_{p}\right) v_{x}^{*} x_{o}^{*}-\left(i \cdot T_{p} \cdot v_{x}^{*}\right)^{2} \\
\sigma_{y_{T}}{ }^{2}= & \sigma_{y_{o}}{ }^{2}+\left(i \cdot T_{p}\right)^{2} \cdot \sigma_{v_{y}}{ }^{2}+2 \cdot\left(i \cdot T_{p}\right) E\left[y_{o} v_{y}\right] \\
& -\left(y_{o}^{*}\right)^{2}-2 \cdot\left(i \cdot T_{p}\right) v_{y} y_{o}^{*}-\left(i \cdot T_{p} \cdot v_{y}^{*}\right)^{2} \\
\sigma_{z_{T}}{ }^{2}= & \sigma_{z_{o}}{ }^{2}+\left(i \cdot T_{p}\right)^{2} \cdot \sigma_{v_{z}}{ }^{2}+2 \cdot\left(i \cdot T_{p}\right)^{E}\left[z_{o} v_{z}\right] \\
& -\left(z_{o}^{*}\right)^{2}-2 \cdot\left(i \cdot T_{p}\right) v_{z}^{*} z_{o}^{*}-\left(i \cdot T_{p} \cdot v_{z}^{*}\right)^{2}
\end{aligned}
$$

From equations (3-27) through (3-29) we see that the variance of the estimated current position has a complex relationship to the number of experiment vectors used and the effective sensor spacing. As these quantities increase, we expect that the variances of the estimated initial positions and velocities will decrease. However, as the number of experiment vectors used and the effective sensor spacing increases, the term $i$ will increase and the variance of the estimate of the current position of the acoustic beacon can also increase. This is because if we project the initial position of the acoustic beacon forward based on the estimated velocity parameters, any errors in the acoustic 
beacon's velocity causes us to move the estimate in the wrong direction. The choice of how many experiment points to collect, or how long we spend collecting data points, will be examined in chapter IV.

As with the stationary beacon, we can test the assumptions made regarding the covariance matrix of the measurement errors. We define the moment matrix as:

$$
M=\sum_{\mu=1}^{n} e_{\mu}^{*} e_{\mu}^{* T}
$$

where the $e_{\mu}^{*}$ 's are the final residual vectors defined as in chapter II. To estimate the covariance of the residuals we take:

$$
\tilde{V}=\frac{6}{n-6} \cdot M
$$

where $\mathrm{n}$ is the number of experiment vectors used in the maximum likelihood estimation. The first term in equation (3-31) corrects for the bias caused by the constrained minimization problem [Bard 1974]. We can compare the results of equation (3-31) with the assumed covariance matrix of the measurement errors to judge if the assumed covariance matrix $\mathrm{V}_{\mu}$ is valid.

\subsection{Summary}

In this chapter we have extended the results of chapter II to include linear motion of the acoustic beacon. We found that the solution to the maximum likelihood estimator for the case of the moving beacon follows the same form as the solution for the case of the stationary beacon. The quantity $(j-i)$ is one of the free parameters of the moving acoustic beacon estimation problem. We define this quantity as the 
effective sensor spacing. This quantity corresponds to the spacing in time between the two signals used to form the experiment vector $\mathrm{w}_{\mu}$. The other free parameter is the number of experiment vectors to use in the parameter estimation problem. In the next chapter we examine the performance of the algorithm described in this chapter for different choices of the two free parameters. 


\section{Chapter IV}

\section{Algorithm Simulations and Tests}

\subsection{Introduction}

In chapters II and III we developed algorithms to solve for the maximum likelihood estimate of an acoustic beacon's position for both the stationary and the moving beacon. In both chapters we were left with choosing how many experiment vectors $\left(w_{\mu}\right)$ to use, and which measurement vectors $\left(m_{\mu}\right)$ to use to define the experiment vectors. We defined the effective element spacing to be the number of pulse repetition intervals $\left(\mathrm{T}_{\mathrm{p}}\right)$ between the measurement vectors that form the experiment vector $w_{\mu}$. In this chapter we examine the performance of the algorithms developed in chapters II and III for different numbers of experiment vectors, and for different effective element spacings. We show that the accuracy of the estimated beacon position $\left(\theta^{*}\right)$ improves, while the variance of the estimated position decreases as the number of experiment vectors used to find the maximum likelihood estimate increases. We also show that the accuracy of the estimated beacon position improves, and the variance of the estimated position decreases with increased effective sensor spacing. This result is similar to the methods mentioned in chapter I.

To test the algorithms of chapters II and III, Monte Carlo simulations were conducted for several different initial positions and velocities of the acoustic beacon. Results from six of the scenarios tested are presented in the following sections. We assume that the measurement errors are as defined in section 2.4. To see how the accuracy of the measured position of receipt $\left(\mathrm{x}_{\mu}, \mathrm{y}_{\mu}\right)$ affects the maximum likelihood 
estimate, we use GPS errors of 3 meters rms and 25 meters rms. These errors approximate the errors of a Differential/P code GPS receiver and a C/A code GPS receiver respectively. For measurement errors in the synchronized time of receipt $t_{\text {sp }}$, we assume the sampling rate of the receiver, $T_{s}$, is 1 millisecond and use equation (216) to define the variance.

\subsection{Synthetic Measurement Vector Generation}

To test the algorithms of chapters II and III, synthetic measurement vectors were generated for several different acoustic beacon positions and velocities. Table 4-1 lists the initial position and velocities for each of the scenarios presented. We assume that the receiving ship moves in a hexagonal search path with legs of 1000 meters. This is a reasonable search path in the general location of the acoustic beacon which is easy to model. The actual path of the receiving ship is not critical,

\begin{tabular}{||c|c|c|c|c|c|c||}
\hline \hline Scenario & $\begin{array}{c}\mathbf{x}_{\mathrm{B}} \\
(\mathbf{m})\end{array}$ & $\begin{array}{c}\mathbf{y}_{\mathrm{B}} \\
(\mathbf{m})\end{array}$ & $\begin{array}{c}\mathbf{z}_{\mathrm{B}} \\
(\mathbf{m})\end{array}$ & $\begin{array}{c}\mathbf{v}_{\mathbf{x}} \\
(\mathbf{m} / \mathbf{s})\end{array}$ & $\begin{array}{c}\mathbf{v}_{\mathbf{y}} \\
(\mathbf{m} / \mathbf{s})\end{array}$ & $\begin{array}{c}\mathbf{v}_{\mathbf{z}} \\
(\mathbf{m} / \mathbf{s})\end{array}$ \\
\hline \hline 1 & 0 & 0 & -2000 & 0 & 0 & 0 \\
\hline 2 & 0 & 866 & -2000 & 0 & 0 & 0 \\
\hline 3 & -1000 & 1000 & -2000 & 0 & 0 & 0 \\
\hline 4 & 0 & 0 & -2000 & 0.5 & -0.5 & 0.25 \\
\hline 5 & 0 & 866 & -2000 & 0.5 & -0.5 & 0.25 \\
\hline 6 & -1000 & 1000 & -2000 & 0.5 & -0.5 & 0.25 \\
\hline
\end{tabular}

Table 4-1

as long as it is not linear. If the receiving ship's path is linear we will be unable to 
accurately resolve the acoustic beacon's position in three dimensions. This is analogous to the inability of a conventional linear array to resolve the direction of arrival of a signal in two dimensions. We restrict the speed of the receiving ship to five meters per second so that flow noise does not become a problem.

Figure 4-1 shows the positions of the acoustic beacon for the scenarios defined in Table 4-1 compared to the path of the receiving ship.

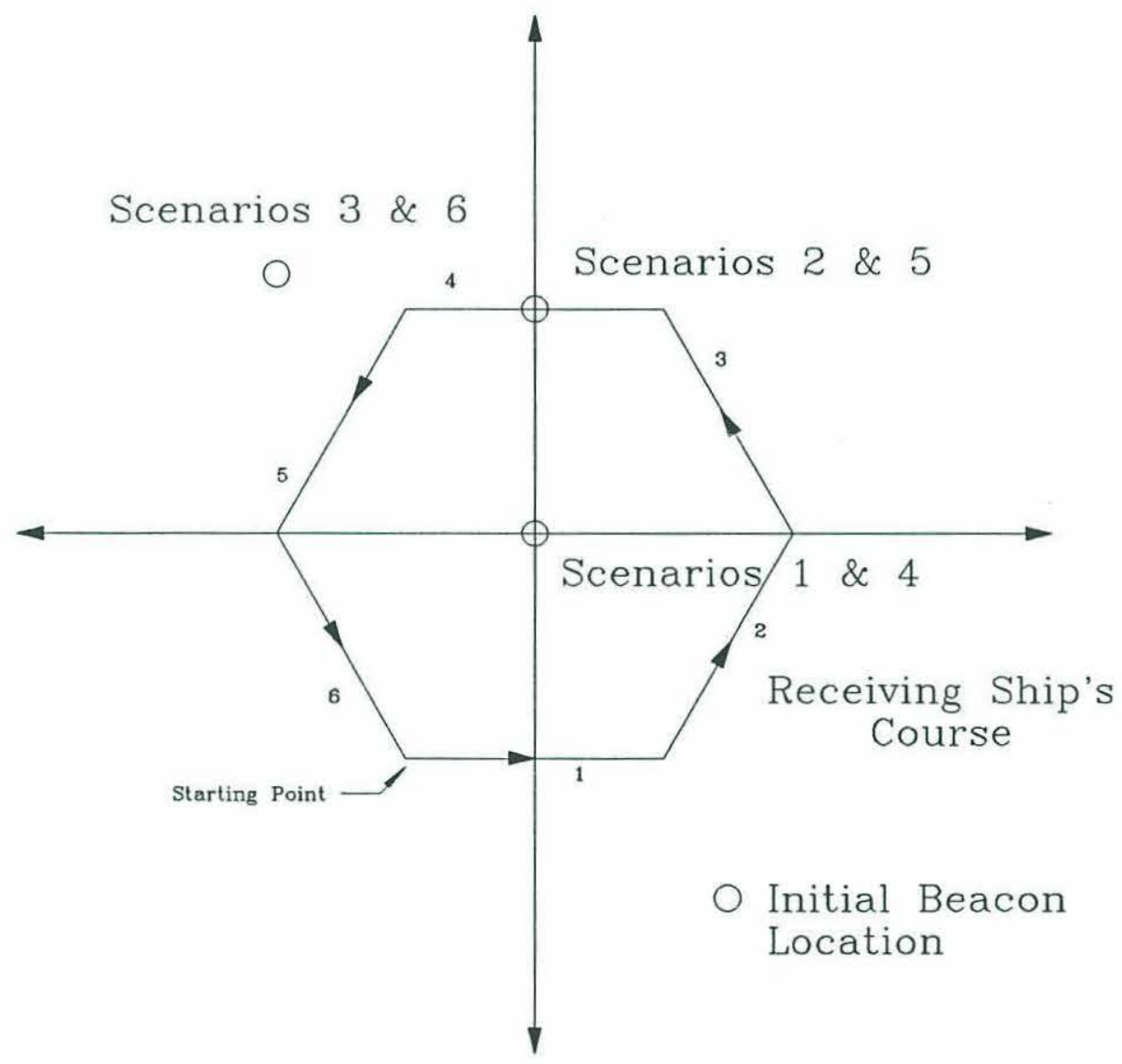

Figure 4-1

Scenarios 1 and 4 corresponds to an acoustic beacon located in the center of the search path, scenarios 2 and 5 correspond to an acoustic beacon located directly below the search path, and scenarios 3 and 6 correspond to an acoustic beacon located outside of 
the search path.

To generate the synthetic measurement vectors $\mathrm{m}_{\mu}$, we solve for the intercept of the receiving ship and the beacon signal. Figure 4-2 shows the intercept geometry.

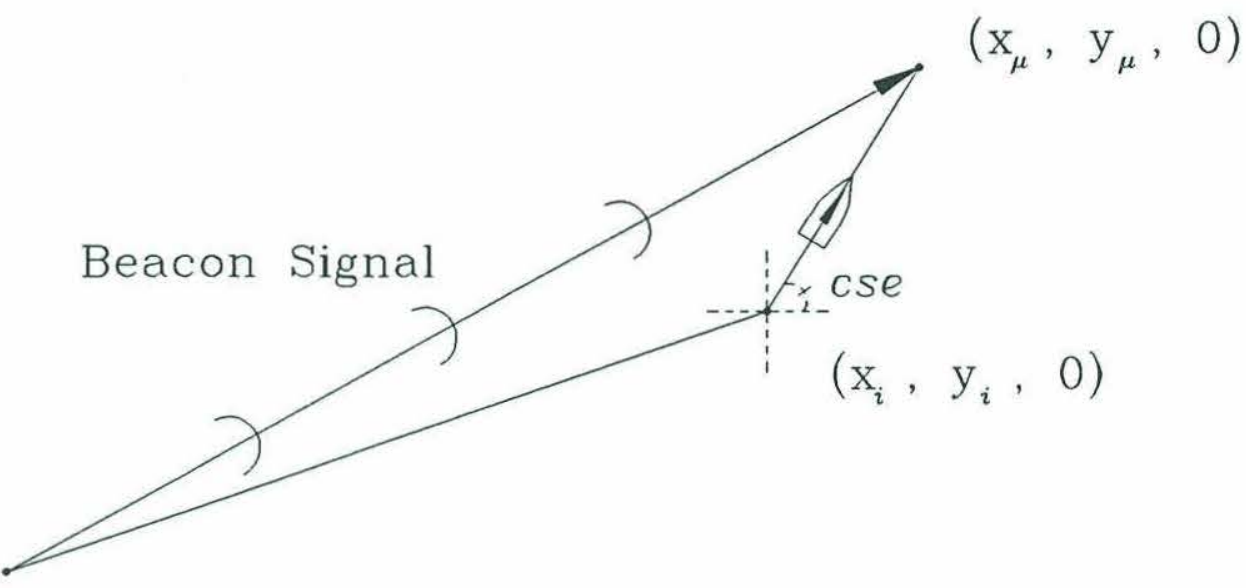

Acoustic Beacon

Location

$\left(x_{B}, y_{B}, z_{B}\right)$

Figure 4-2

The position $\mathrm{x}_{\mathrm{i}}, \mathrm{y}_{\mathrm{i}}$ is the location of the receiving ship when the beacon signal is transmitted, and the position $\mathrm{x}_{\mu}, \mathrm{y}_{\mu}$ is the location of the ship when the beacon signal is received. Using a homogeneous ocean model and a direct path propagation mode, we calculate the synchronized time of receipt by finding the positive root of:

$$
\alpha \cdot t^{2}+\beta \cdot t+\gamma=0
$$

where:

$$
\begin{aligned}
& \alpha=s^{2}-C^{2} \\
& \beta=2 \cdot s \cdot\left[\cos (\operatorname{cse} e) \cdot\left(x_{i}-x_{B}\right)+\sin (\operatorname{cs} e) \cdot\left(y_{i}-y_{B}\right)\right] \\
& \gamma=\left(x_{i}-x_{B}\right)^{2}+\left(y_{i}-y_{B}\right)^{2}+z_{B}^{2}
\end{aligned}
$$


Here $s$ is the speed of the receiving ship in meters per second, C is the speed of sound in sea water, and cse is the course of the receiving ship in degrees. It is assumed that the receiving ship does not change its course for the duration of the calculation. Once we have the synchronized time of receipt, we calculate the position of receipt $x_{\mu}, y_{\mu}$ using:

$$
\begin{aligned}
& x_{\mu}=x_{i}+t_{s \mu} \cdot s \cdot \cos (c s e) \\
& y_{\mu}=y_{i}+t_{s \mu} \cdot s \cdot \sin (c s e)
\end{aligned}
$$

To find the next synchronized time of receipt and position of receipt $\left(x_{\mu}, y_{\mu}\right)$ we update the acoustic beacon's position based on its velocity:

$$
\begin{aligned}
& x_{B}=x_{B}+T_{p} \cdot v_{x} \\
& y_{B}=y_{B}+T_{p} \cdot v_{y} \\
& z_{B}=z_{B}+T_{p} \cdot v_{z}
\end{aligned}
$$

where $T_{p}$ is the pulse repetition interval of the acoustic beacon. For the results presented in the following sections we assume the acoustic beacon has a pulse repetition interval of 2 seconds. Next we find the location of the receiving ship when the new beacon signal is transmitted using:

$$
\begin{aligned}
& x_{i}=x_{i}+T_{p} \cdot s \cdot \cos (c s e) \\
& y_{i}=y_{i}+T_{p} \cdot s \cdot \sin (c s e)
\end{aligned}
$$

We then solve for the intercept of the beacon signal and the receiving ship using equations (4-1) and (4-2). We continue in this manner until we have generated a sufficient number of measurement vectors $\mathrm{m}_{\mu}$. Once we have generated the 
measurement vectors, we add normally distributed random error terms to each measurement based upon the errors discussed in section 2.4 .

\subsection{Stationary Acoustic Beacon}

In this section we examine the performance of the algorithm given in Figure 2-4. Figures 4-3 through 4-50 contain the results of the Monte Carlo simulations conducted for scenarios 1 through 3. For each scenario, the initial value of the estimated parameter vector $\theta^{*}$ is taken as:

$$
\theta_{0}^{*}=\left[\begin{array}{lll}
0 & 0 & -2500
\end{array}\right]^{T}
$$

The feasible region is defined by Range $\max _{\max }=$ Depth $_{\max }=5000$ meters. If the maximum likelihood estimate found using the algorithm of chapter II is outside the feasible region, the corresponding entries are left blank. To find a solution for these cases, we need to start with a different initial parameter vector $\theta^{*}$, or use more experiment vectors.

To test the effect of increasing the number of experiment vectors $(n)$, we solve for the estimated parameter vector $\theta^{*}$ using $n$ ranging from 5 to 50 . We fix the effective sensor spacing at 45 pulse repetition intervals by using every $45^{\text {th }}$ received beacon signal in defining the experiment vectors. To test the effects of increasing the effective sensor spacing, we solve for the estimated position vector using effective sensor spacings ranging from 2 to 90 pulse repetition intervals. For this case we fix the number of experiment vectors used to 25 . We conduct each experiment using GPS errors of 3 meters rms and 25 meters rms. The results of the estimation algorithm for GPS errors of 3 meters rms are shown by x's on the plots, while the results of the 
estimation algorithm for GPS errors of 25 meters rms are shown by o's.

\subsubsection{Scenario 1 - Stationary Acoustic Beacon Located at $\theta=\left[\begin{array}{lll}0 & 0 & -2000\end{array}\right]^{\mathrm{T}}$}

Figures 4-3 through 4-10 show the results of the maximum likelihood estimation algorithm for different numbers of experiment vectors, and figures 4-11 through 4-18 show the results of the maximum likelihood estimation algorithm for different effective sensor spacings for scenario 1 . We see that in general, as the number of experiment vectors used in the maximum likelihood estimation problem increases, the accuracy of the estimated beacon position improves, and the standard deviation of the estimated position decreases. Also, as the effective sensor spacing increases, the accuracy of the estimated beacon position improves, and the standard deviation of the estimated position decreases. For both cases, the position errors and the standard deviation of the estimates initially decrease rapidly as the receiving ship spans more of its two dimensional search path. However after the number of experiment vectors used reaches approximately 20 , or after the effective sensor spacing reaches approximately 35 , the errors and the standard deviations decrease at much slower rate. At this point the receiving ship has completed two legs of the search path, and spans enough of the two dimensional search path to form a decent estimate of the acoustic beacon's position. 

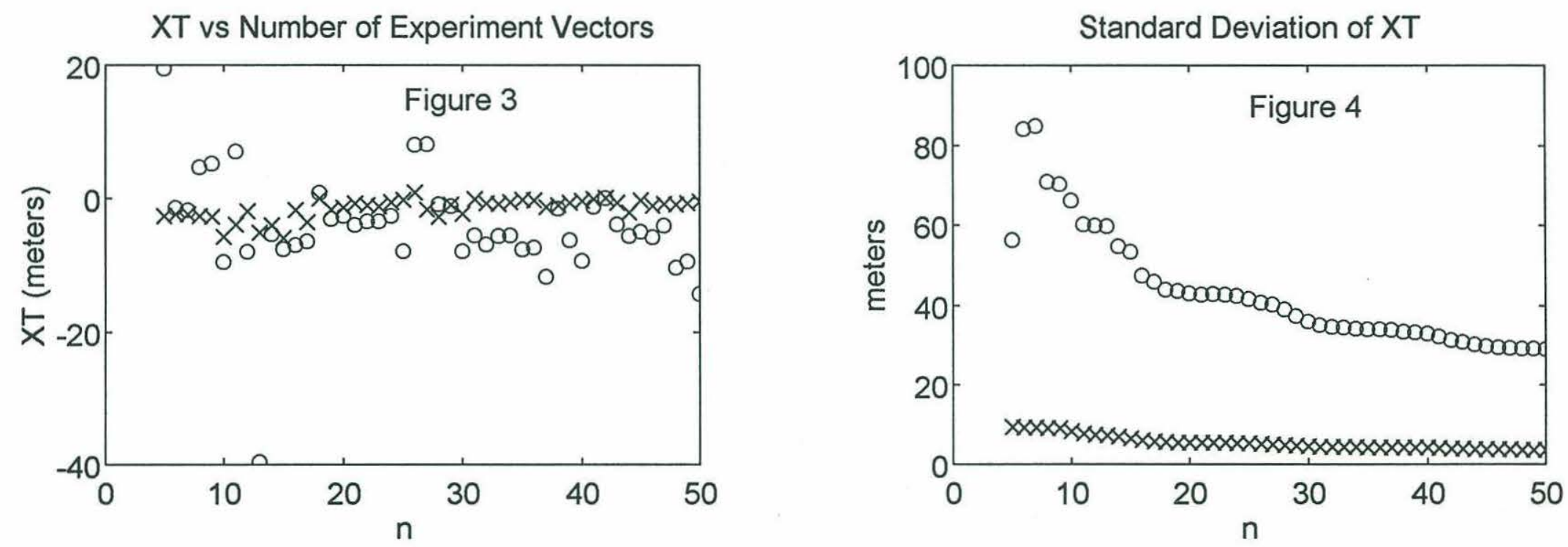

오
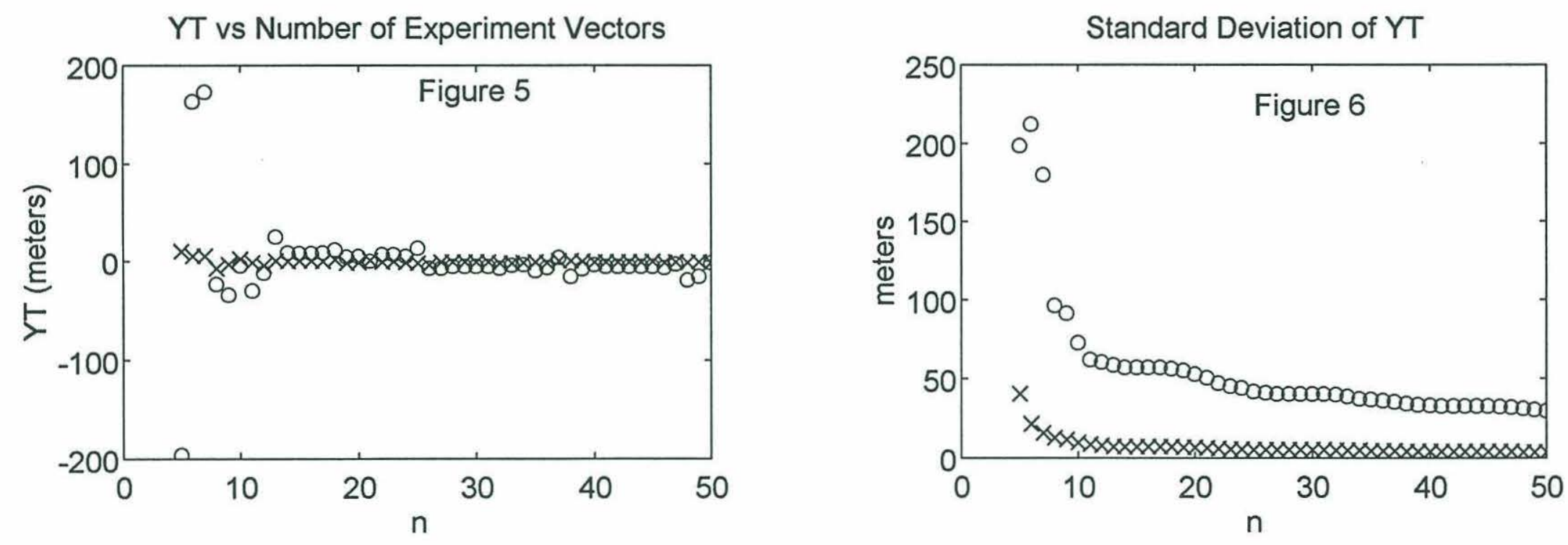

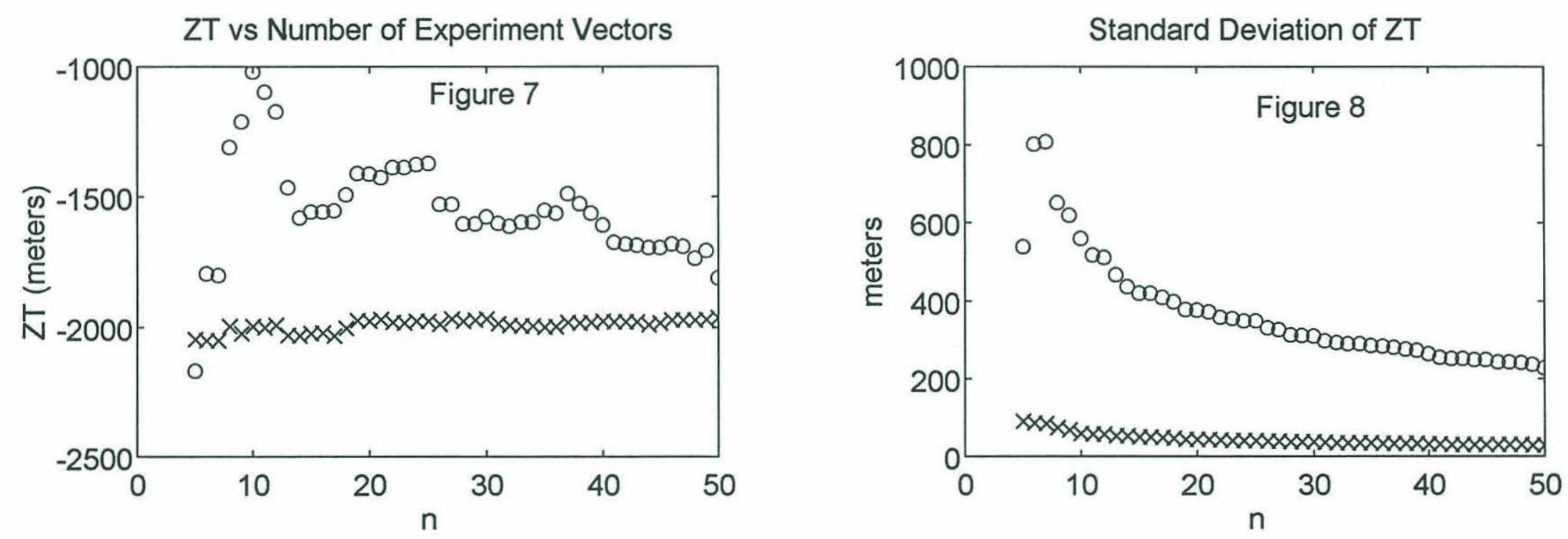

$\infty$
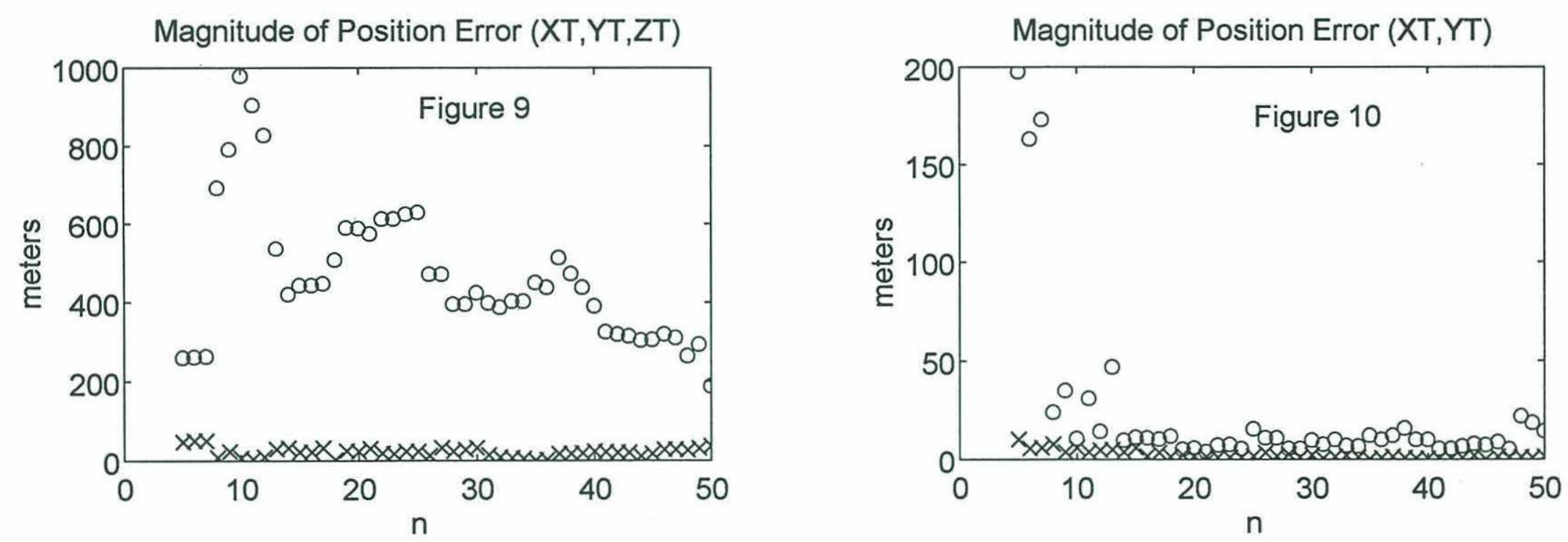

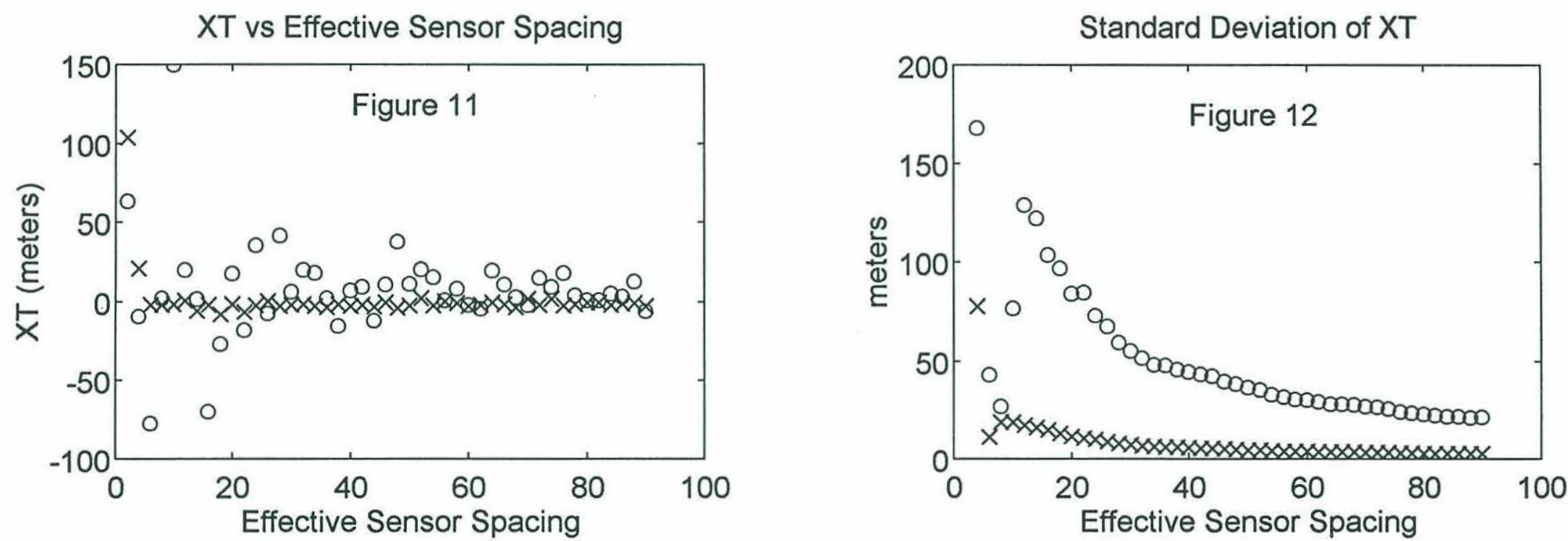

옹
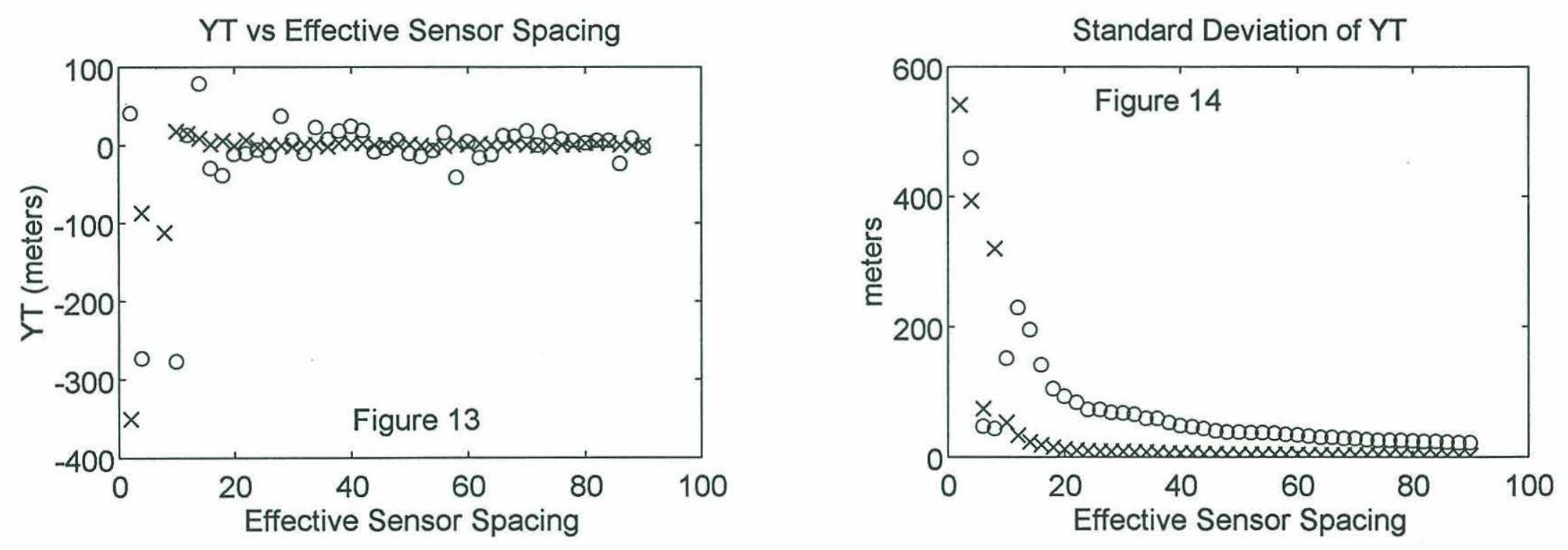

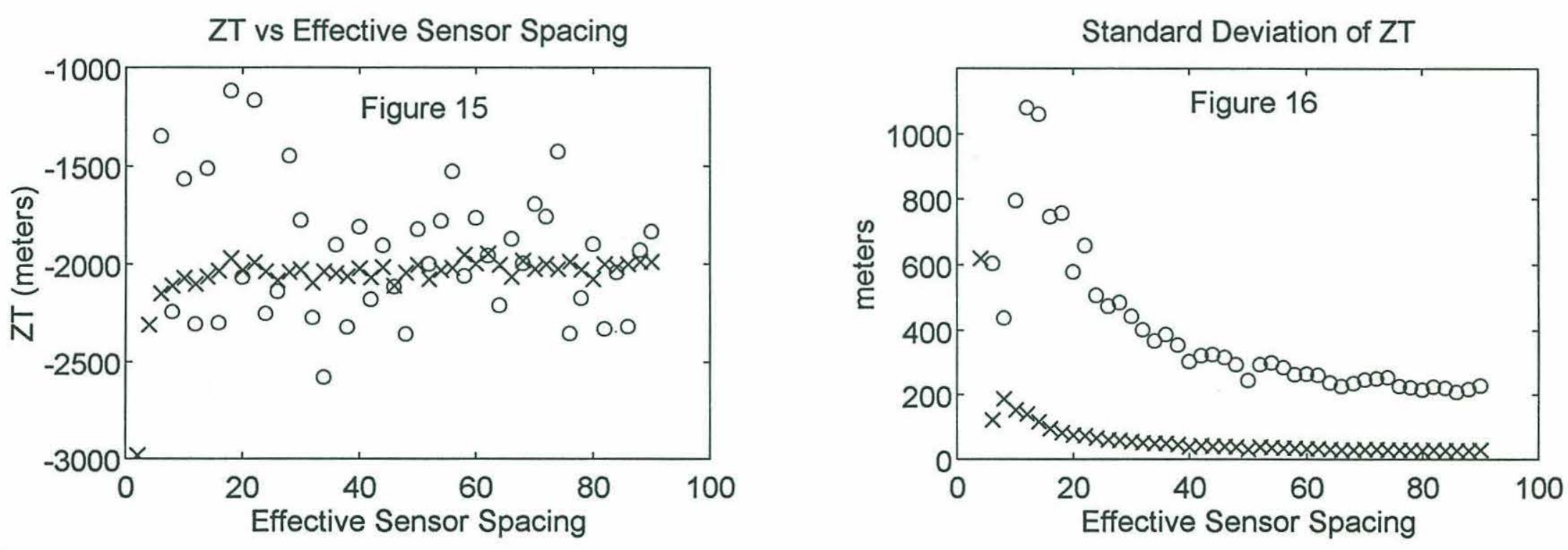

ㄱ. Magnitude of Position Error (XT,YT,ZT)
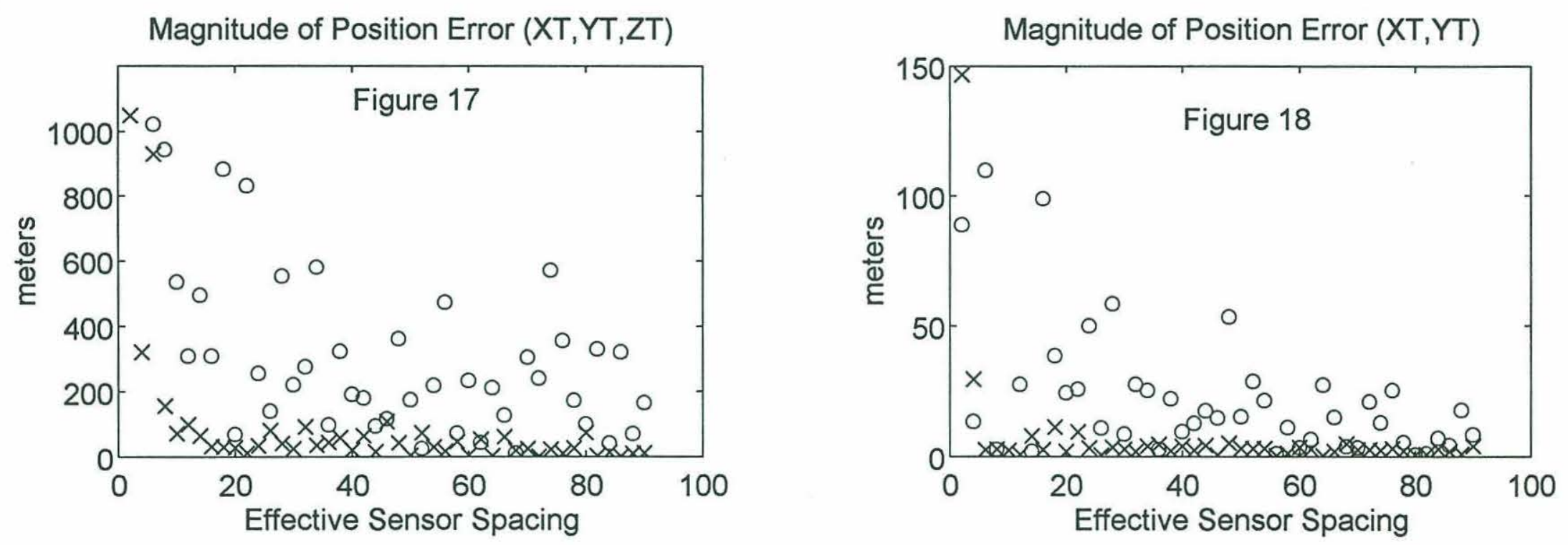


\subsubsection{Scenario 2 - Stationary Acoustic Beacon Located at $\theta=\left[\begin{array}{lll}0 & 866-2000\end{array}\right]^{\mathrm{T}}$}

Figures 4-19 through 4-26 show the results of the maximum likelihood estimation algorithm for different numbers of experiment vectors, and figures 4-27 through 4-34 show the results of the maximum likelihood estimation algorithm for different effective sensor spacings for scenario 2. Like scenario 1 , as the number of experiment vectors used in the maximum likelihood estimation problem increases, the accuracy of the estimated beacon position improves, and the standard deviation of the estimated position decreases. Also like scenario 1, as the effective sensor spacing increases, the accuracy of the estimated beacon position improves, and the standard deviation of the estimated position decreases. Again we see an initial rapid decrease in the errors and standard deviations which levels off after the number of experiment vectors used reaches approximately 20 , or the effective sensor spacing reaches approximately 35. As in scenario 1 , these values correspond to when the receiving ship completes the second leg of the search path.

Unlike scenario 1, we see a significant improvement in the estimated position (especially when using 25 meter GPS errors) when the number of experiment vectors used is greater then 40 , or the effective sensor spacing is greater then 65 . At this point the receiving ship passes directly over the acoustic beacon and begins moving away from the beacon on the fourth leg of the search path. To see why we get this improvement, we can define a vertical plane as the plane orthogonal to the $x-y$ plane that passes through the acoustic beacon's position and the starting point of the leg of the search path that the receiving ship is currently on. In general, it was found that if 
the receiving ship moves such that it stays in this vertical plane, significant improvements to the estimated beacon location can be made. This is because as the receiving ship moves in the same vertical plane relative to the acoustic beacon, the localization problem is essentially a two dimensional problem, and the range differences between measurement vectors is maximized for a given ship's velocity. The same is true if the acoustic beacon and the receiving ship are in the same horizontal plane. 

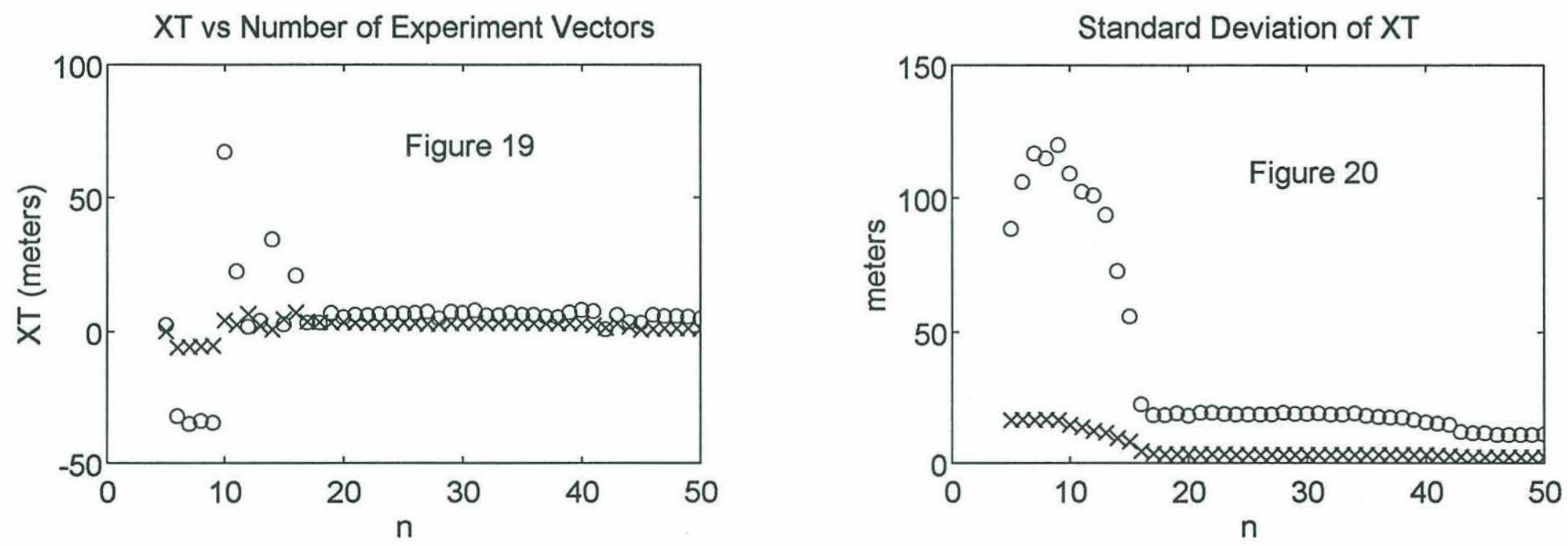

w
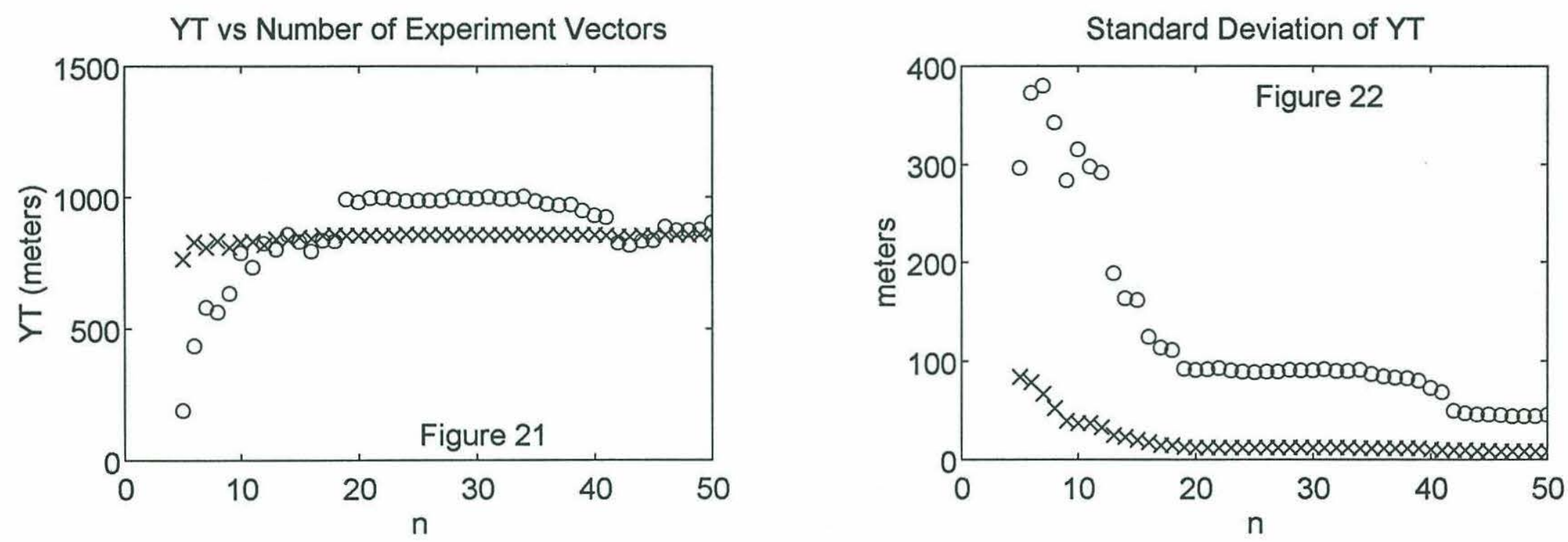

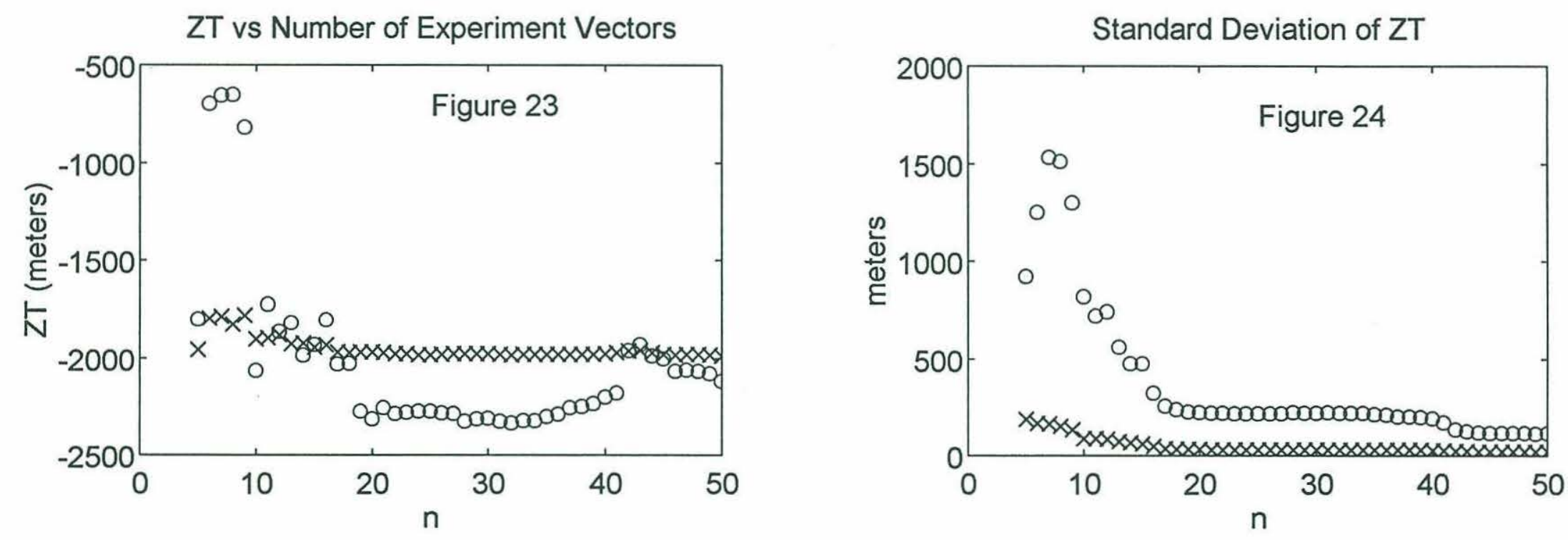

$\mathbb{D}$
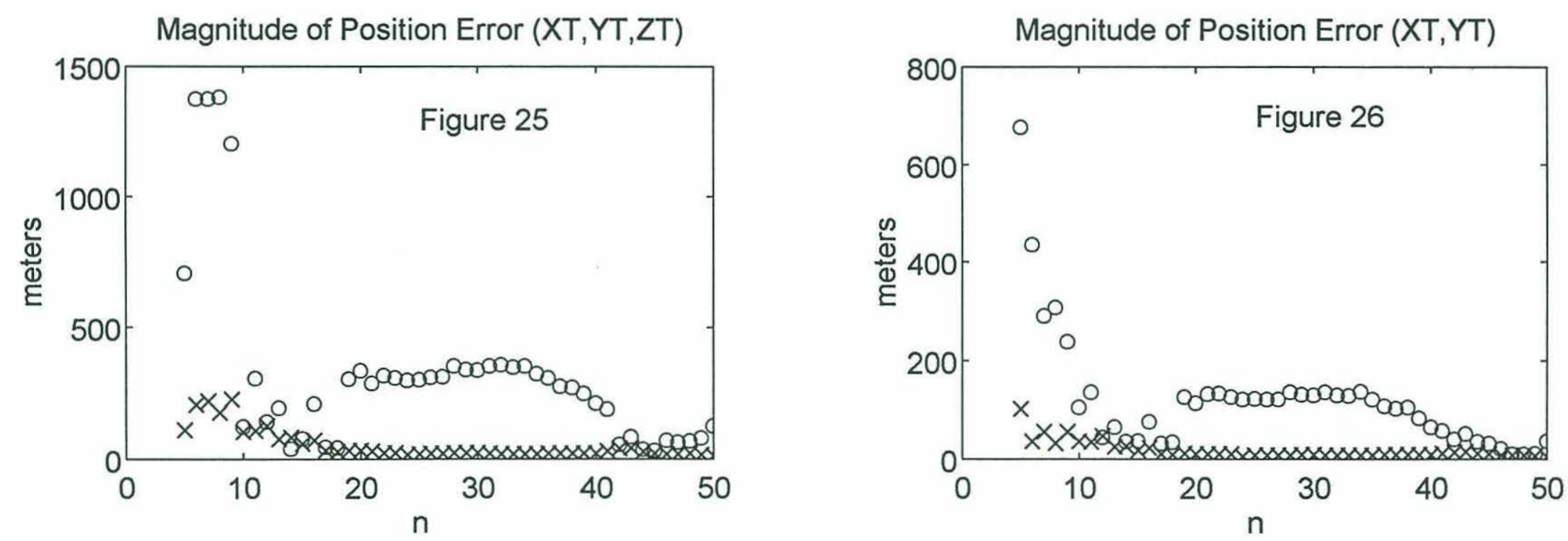

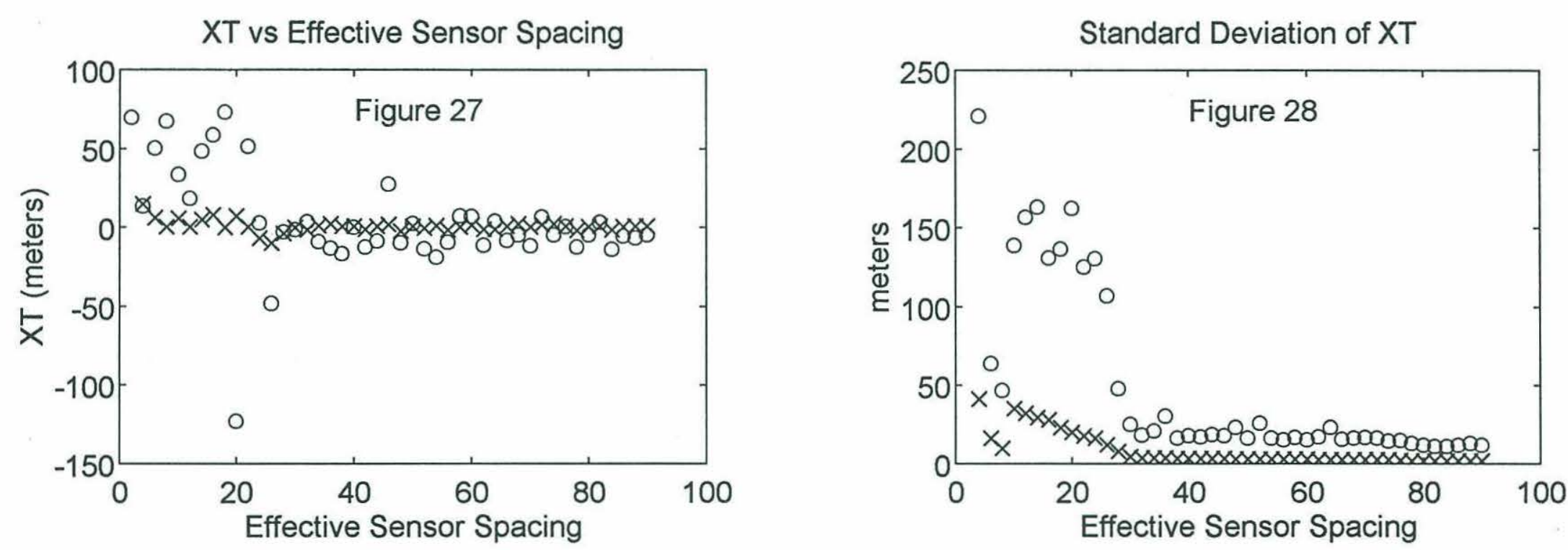

जै
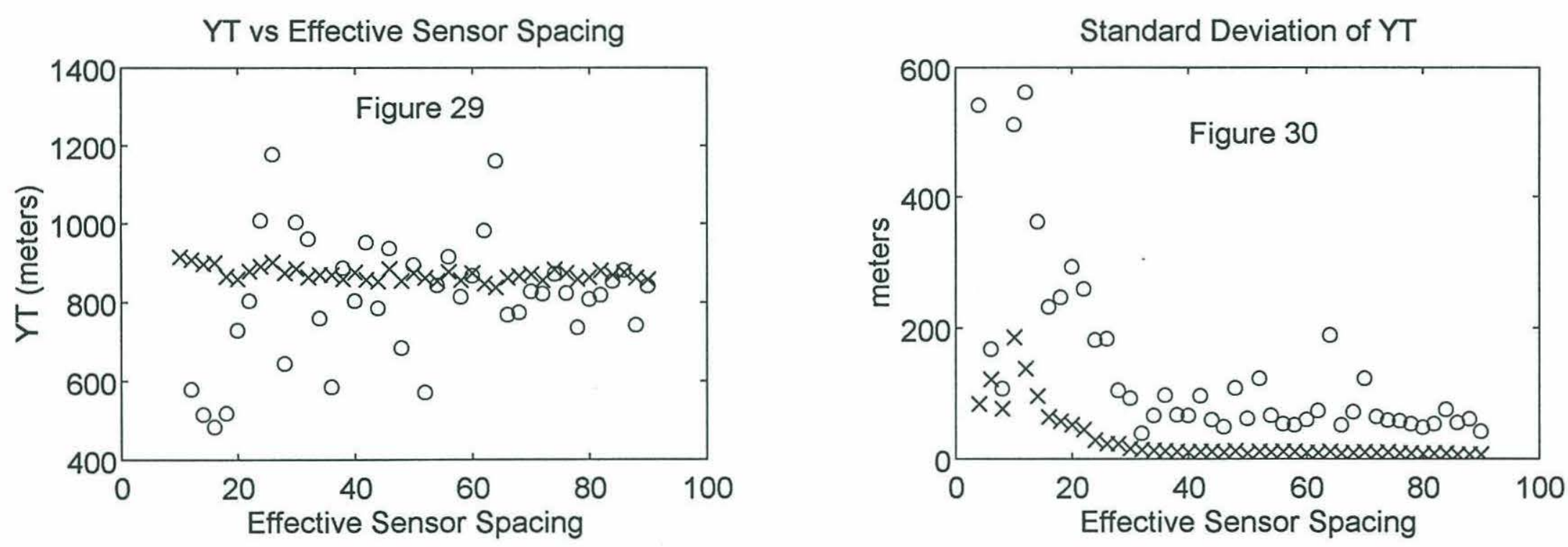

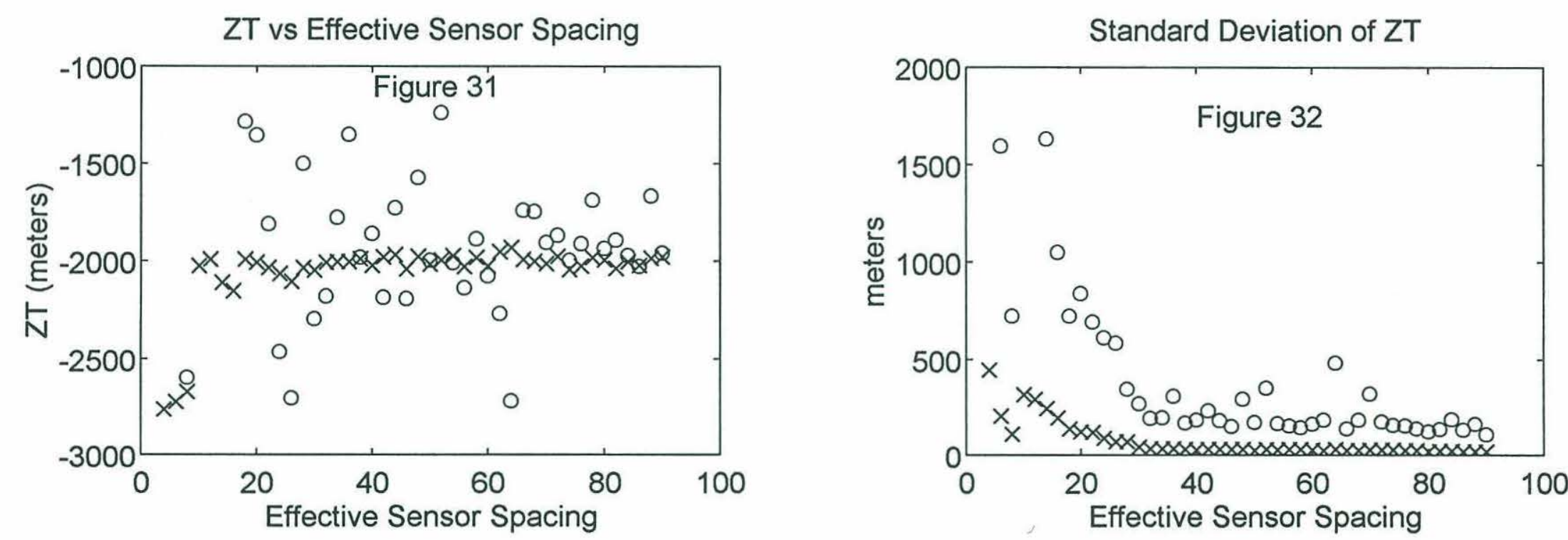

ส้
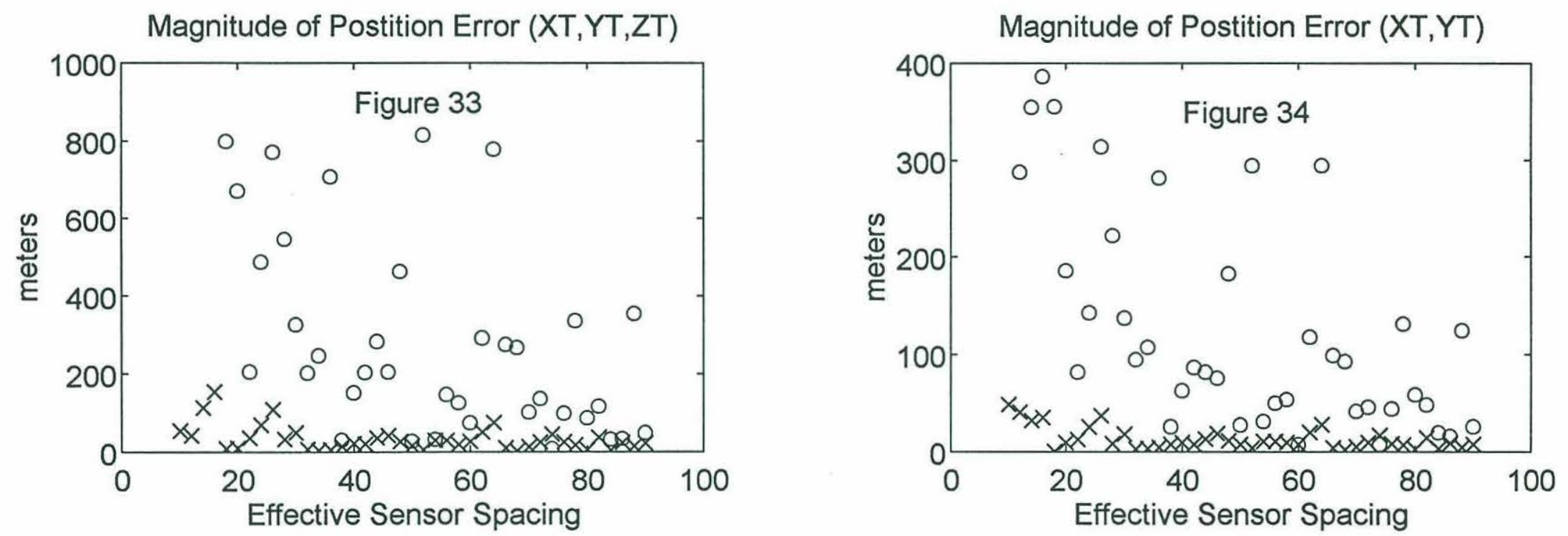


\subsubsection{Scenario 3 - Stationary Acoustic Beacon Located at $\theta=\left[\begin{array}{lll}-1000 & 1000-2000\end{array}\right]^{\mathrm{T}}$}

Figures 4-35 through 4-42 show the results of the maximum likelihood estimation algorithm for different numbers of experiment vectors, and figures 4-43 through 4-50 show the results of the maximum likelihood estimation algorithm for different effective sensor spacing for scenario 3. Like the first two scenarios, as the number of experiment vectors used in the maximum likelihood estimation problem increases, the accuracy of the estimated beacon position improves, while the standard deviation of the estimated position decreases. Also, as the effective sensor spacing increases, the accuracy of the estimated beacon position improves, while the standard deviation of the estimated position decreases. Again we see an initial rapid decrease in the errors and standard deviations which levels off after the number of experiment vectors used reaches approximately 20 , and the effective sensor spacing reaches approximately 35. As in the first two scenarios, these values correspond to when the receiving ship completes the second leg of the search path.

In this scenario, we see a significant improvement in the location estimate when the number of experiment vectors used is greater then 45 , or when the effective element spacing is greater then 75. At this point the receiving ship passes through the closest point of approach to the acoustic beacon on the fifth leg of the search path. In general, it was found that the estimate of the acoustic beacon's location improves as the receiving ship passes through closest points of approach to the acoustic beacon along the search path. 

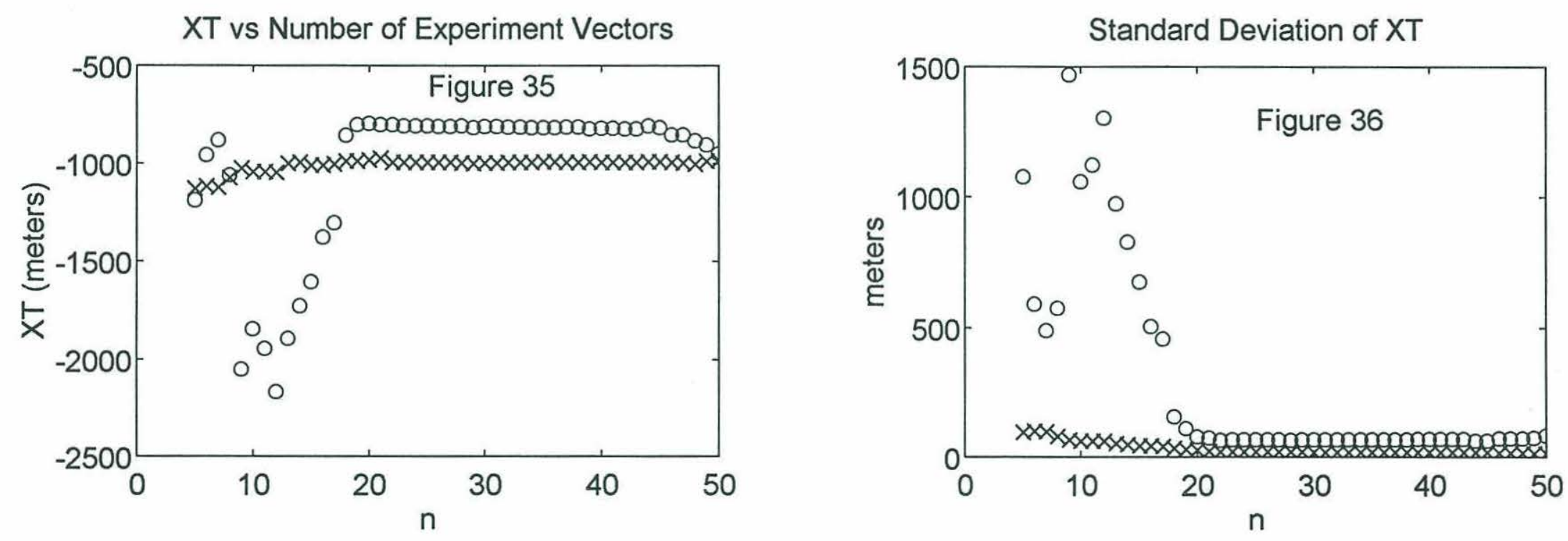

क
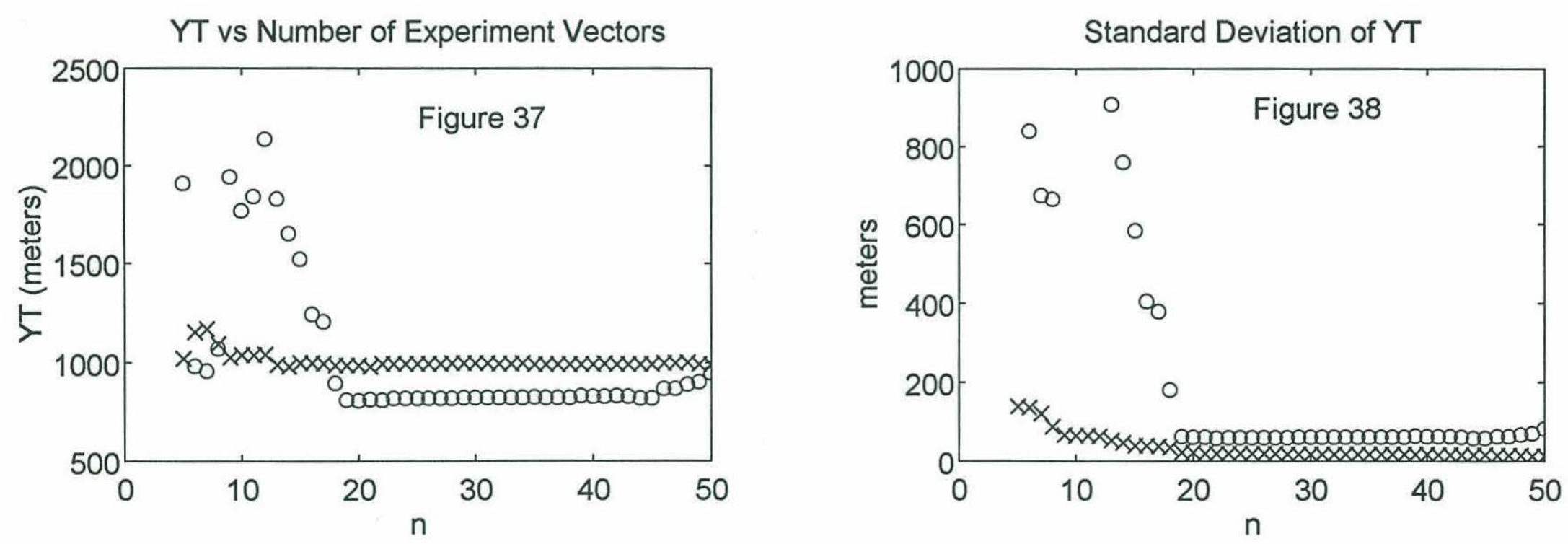

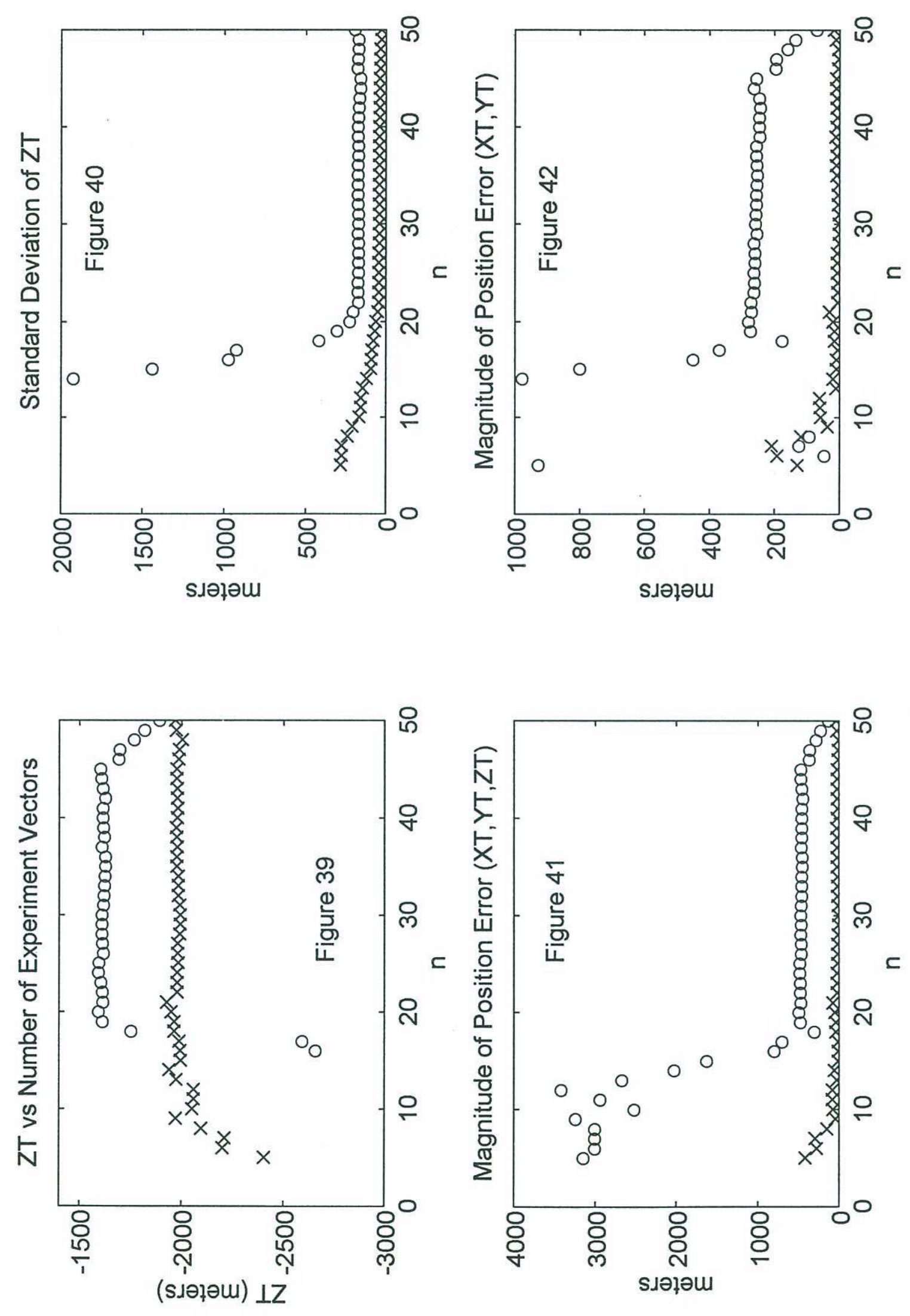


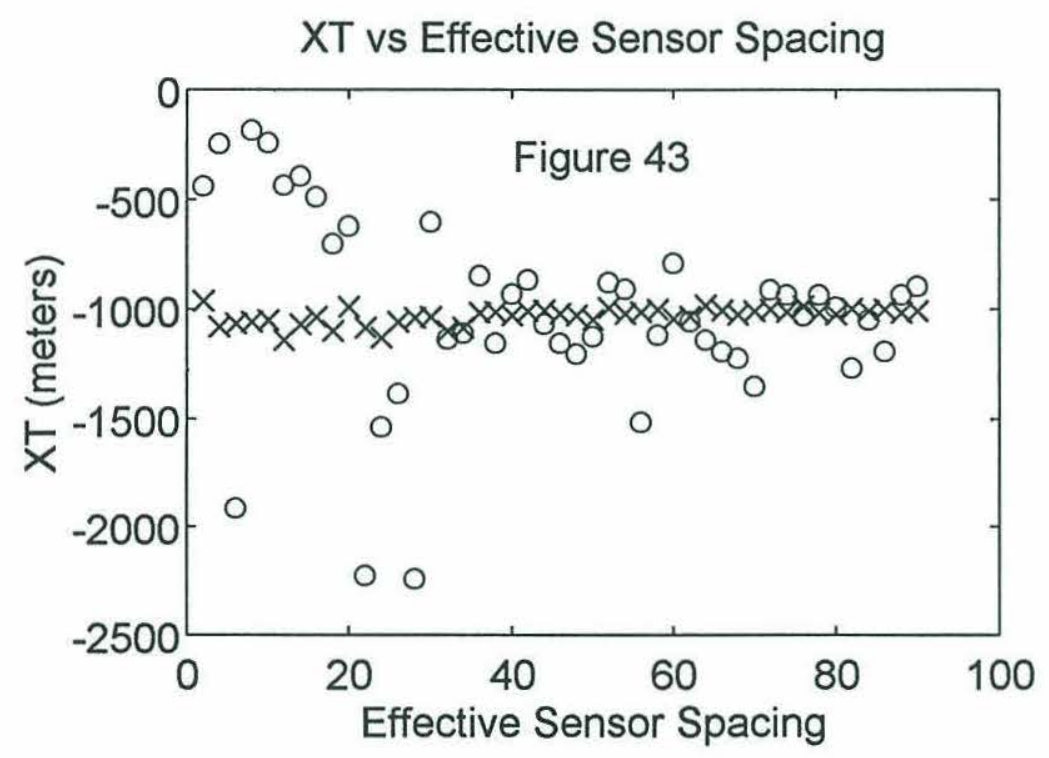

ஃ
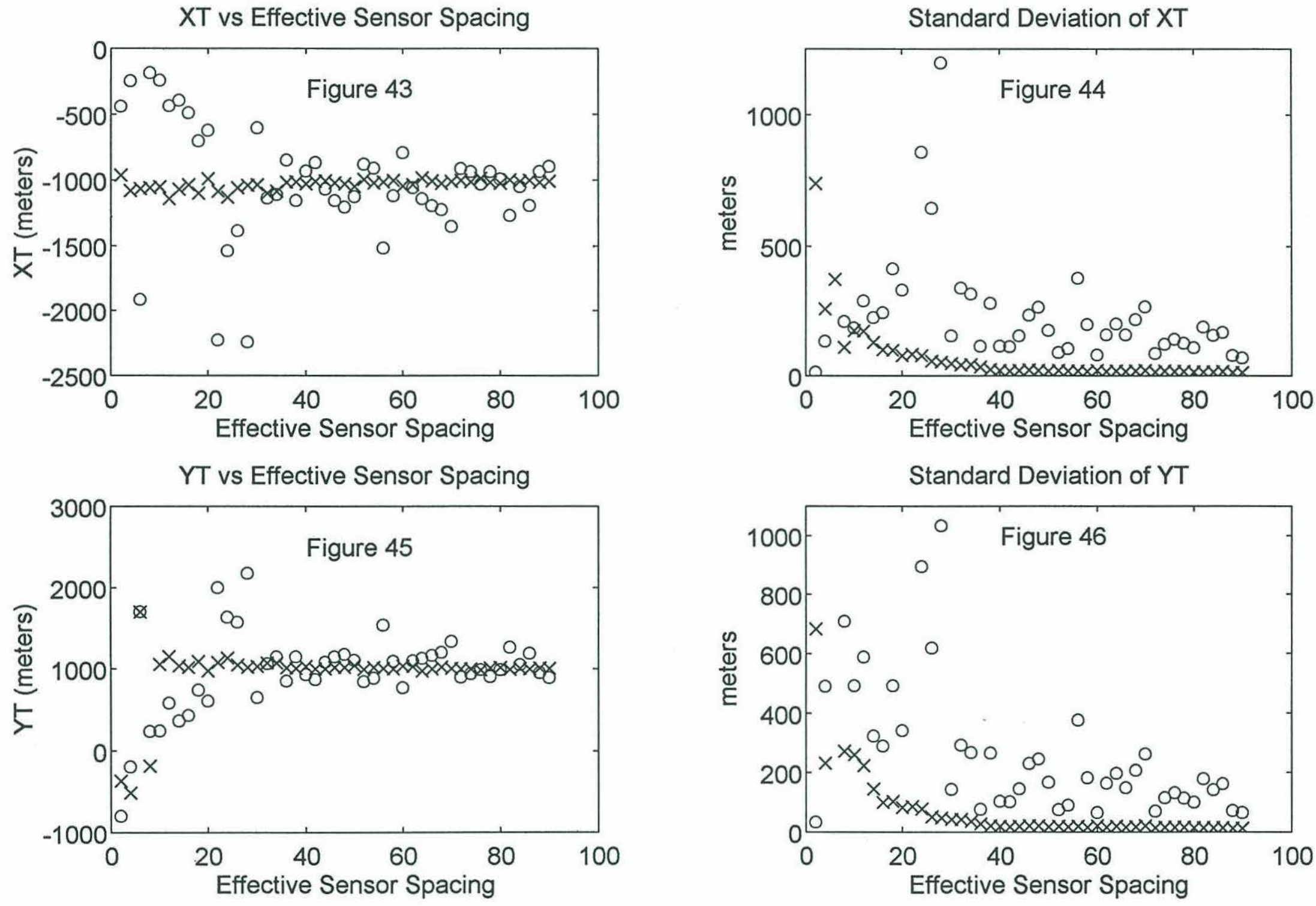

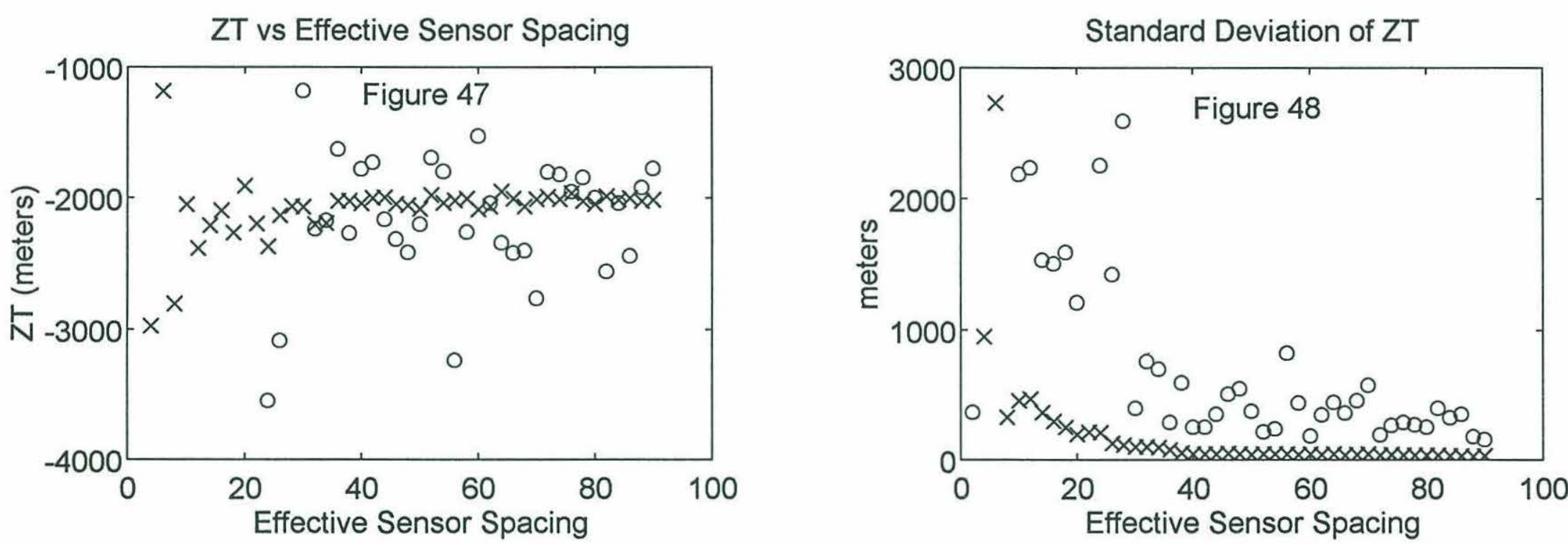

$\stackrel{\infty}{\sim}$
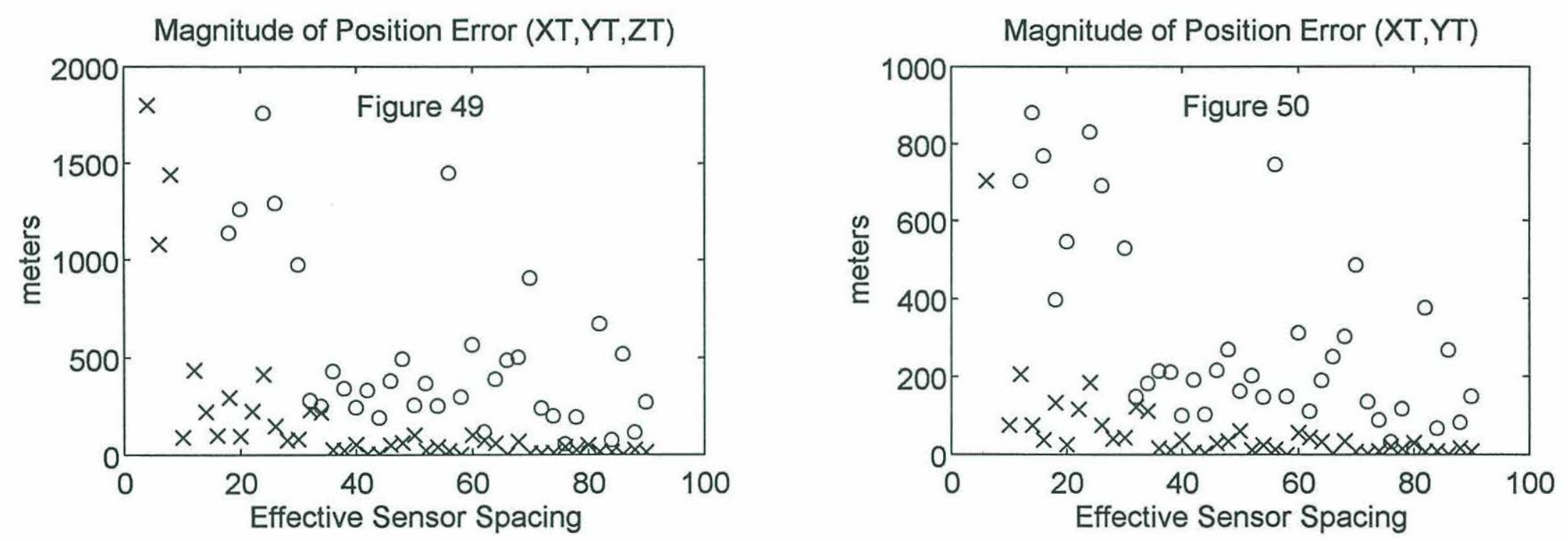


\subsubsection{Stationary Acoustic Beacon Summary}

From scenarios 1 through 3 we showed that as the number of experiment vectors used in the maximum likelihood estimation problem increases, the accuracy of the estimated beacon position improves, while the standard deviation of the estimated position decreases. Also, as the effective sensor spacing increases, the accuracy of the estimated beacon position improves, while the standard deviation of the estimated position decreases. We also showed that as the receiving ship passes through a closest point of approach, the estimated position of the acoustic beacon improves. This is why the estimate of acoustic beacon's location in scenario 1 is better then the estimates obtained for the other two scenarios. In scenario 1, each leg of the search path passes through a closest point of approach. This is not true for the other two scenarios. In all of the scenarios, we see that the depth of the acoustic beacon is the least accurately estimated parameter. This is because we are attempting to locate the acoustic beacon in three dimensions, while the receiving ship only moves in two dimensions. Finally, we see that the accuracy of the estimated acoustic beacon position obtained from using GPS errors of 3 meters is approximately an order of magnitude better then the estimated acoustic beacon position obtained from using GPS errors of 25 meters.

These observations suggest that to obtain the best possible estimate of the acoustic beacon's location, we need to use a large number of experiment vectors with a large effective sensor separation, and we need to use a Differential/P code GPS receiver. The problem with this is that the time it takes to collect the measurement vectors used to form the experiment vectors increases with increasing sensor spacing 
and increasing numbers of experiment vectors. As an example, with an effective sensor spacing of 45 pulse repetition intervals, a pulse repetition interval of 2 seconds, and using 50 experiment vectors it takes over 76 minutes to collect the required measurement vectors. If time is an issue, then we must use fewer experiment vectors and shorter effective sensor spacings. From looking at the results of the simulations presented above, for the hexagonal search path of figure 4-1 and for a pulse repetition interval of 2 seconds, the minimum required number of experiment vectors is 20 , and the minimum effective sensor spacing is 35 . With these values we span enough of the two dimensional search path to obtain a decent estimate of the acoustic beacon's location,

\subsection{Moving Acoustic Beacon}

In this section we examine the performance of the algorithm given in Figure 3-1. Figures 4-51 through 4-146 contain the results of the Monte Carlo simulations conducted for scenarios 4 through 6 . For each scenario, the initial value of the estimated parameter vector $\theta^{*}$ is taken as:

$$
\theta_{o}^{*}=\left[\begin{array}{llllll}
0 & 0 & -2500 & 0 & 0 & 0
\end{array}\right]^{T}
$$

The feasible region is defined by Range $\mathrm{m}_{\max }=$ Depth $_{\max }=5000$ meters, and $\mathrm{v}_{\mathrm{x} \max }=$ $\mathrm{v}_{\mathrm{ymax}}=\mathrm{v}_{\mathrm{zmax}}=5$ meters per second. If the maximum likelihood estimate found using the algorithm of chapter III is outside the feasible region, the corresponding entries are left blank. To find a solution for these cases, we need to start with a different initial parameter vector $\theta$, or use more experiment vectors.

To test the effect of increasing the number of experiment vectors ( $n$ ), we solve 
for the estimated parameter vector $\theta^{*}$ using $n$ ranging from 10 to 50 . We fix the effective sensor spacing at 60 pulse repetition intervals by using every $60^{\text {th }}$ received beacon signal in defining the experiment vectors. To test the effect of increasing the effective sensor spacing, we solve for the estimated position vector using effective sensor spacings ranging from 2 to 90 pulse repetition intervals. For this case we fix the number of experiment vectors used to 30 . We conduct each experiment using GPS errors of 3 meters rms and 25 meters rms. The results of the estimation algorithm for GPS errors of 3 meters rms are shown by x's on the plots, while the results of the estimation algorithm for GPS errors of 25 meters rms is shown by o's.

\subsubsection{Scenario 4 - Moving Acoustic Beacon Located at:}

$$
\theta=\left[\begin{array}{llllll}
0 & 0 & -2000 & 0.5 & -0.5 & 0.25
\end{array}\right]^{\mathrm{T}}
$$

Figures 4-51 through 4-66 show the results of the maximum likelihood estimation algorithm for different numbers of experiment vectors, and figures 4-67 through 4-82 show the results of the maximum likelihood estimation algorithm for different effective sensor spacings for scenario 4 . Similar to the stationary acoustic beacon scenarios, we see that as the number of experiment vectors used in the maximum likelihood estimation problem increases, the accuracy of the estimated position and velocities of the acoustic beacon improves, while the standard deviation of the estimated position and velocities decrease. We also see that as the effective sensor spacing increases, the accuracy of the estimated position and velocities of the acoustic beacon improves, and the standard deviation of the estimated position decreases. As with the stationary acoustic beacon, the position and velocity errors 
initially decrease rapidly, however, after the number of experiment vectors used reaches approximately 30 , or the effective sensor spacing reaches approximately 45 , the errors and the standard deviations decrease much slower.

Figures 4-61 through 4-66 and figures 4-77 through 4-82 show the estimation algorithm's ability to track the linearly moving acoustic beacon. We see that projected current positions follow the linearly moving acoustic beacon fairly well when using 3 meter GPS errors. We also see that the standard deviation of the projected current positions decrease as the number of experiment vectors used increases and the effective sensor spacing increases. However, we notice in a few cases the standard deviations increase slightly for increasing numbers of experiment vectors used and increasing effective sensor spacing. This is a result of projecting the current position forward in time using equation 3-19. 

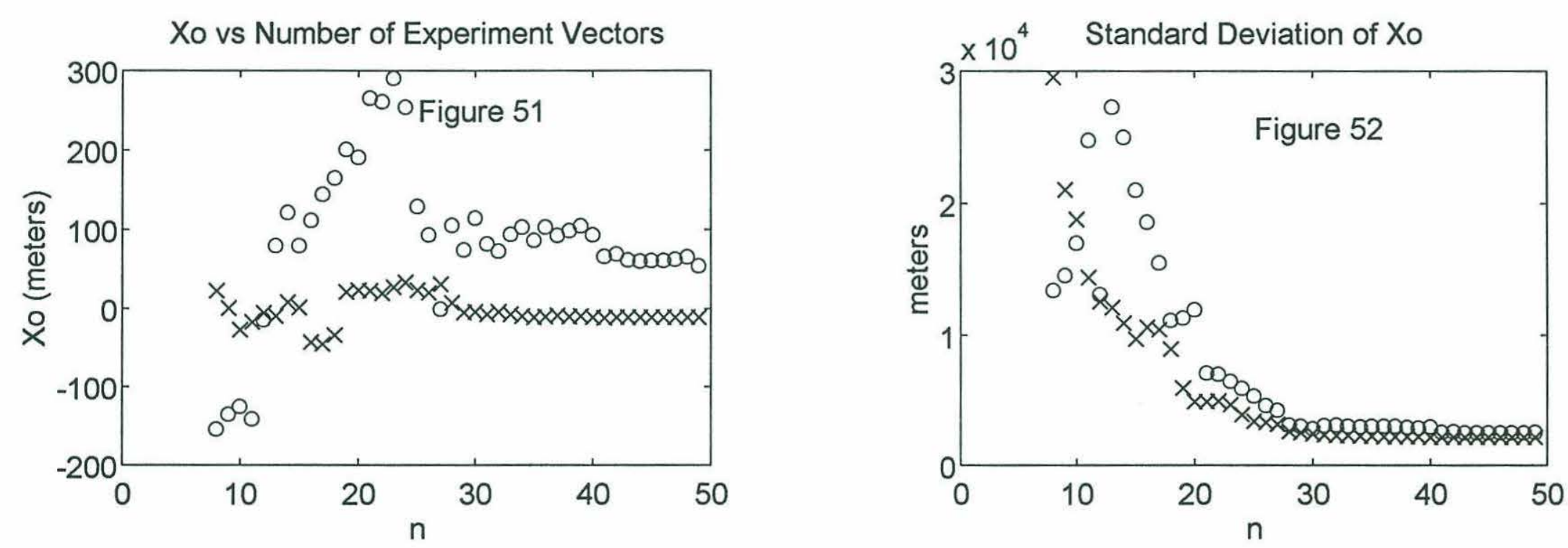

$\infty$
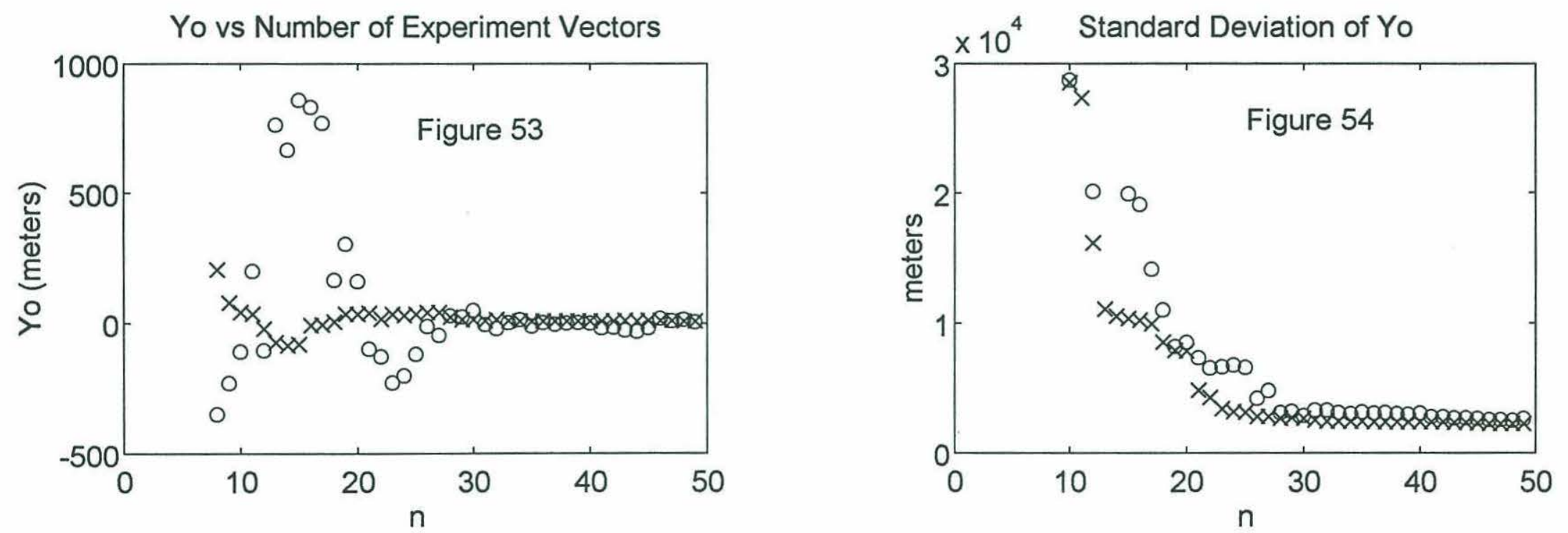


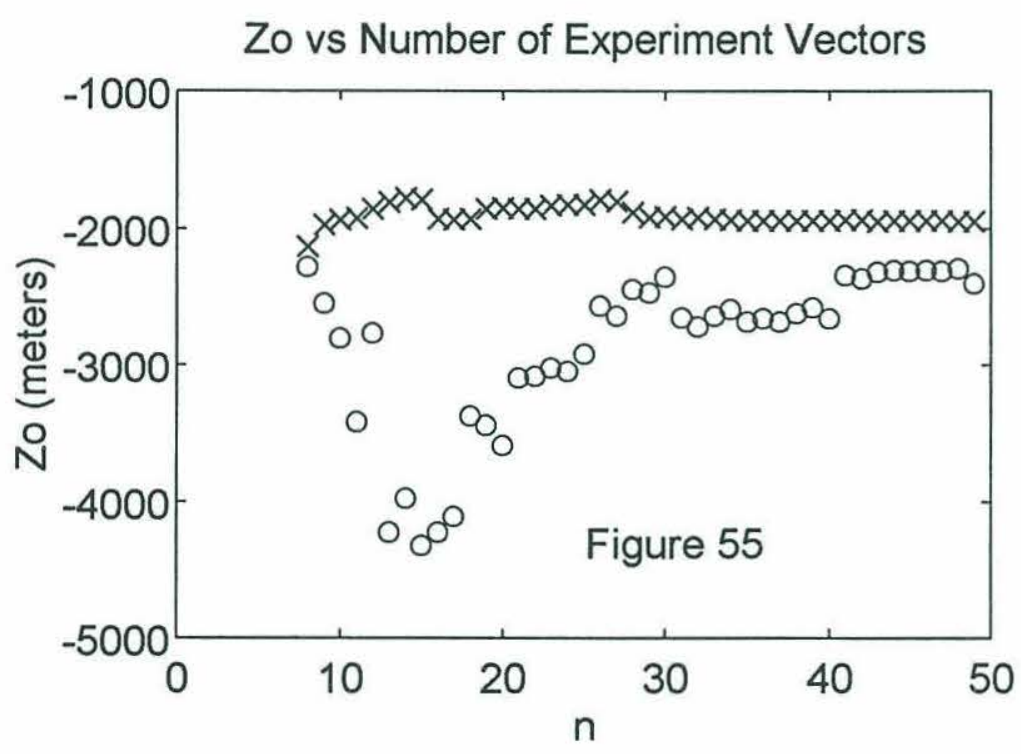

$\stackrel{\infty}{\sim}$

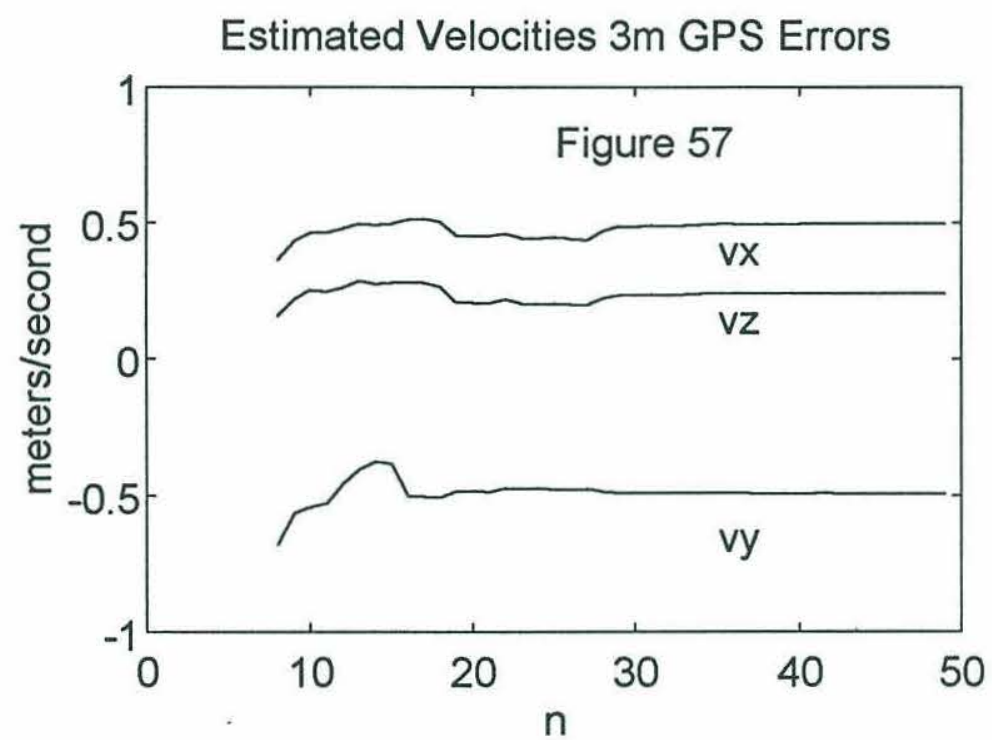

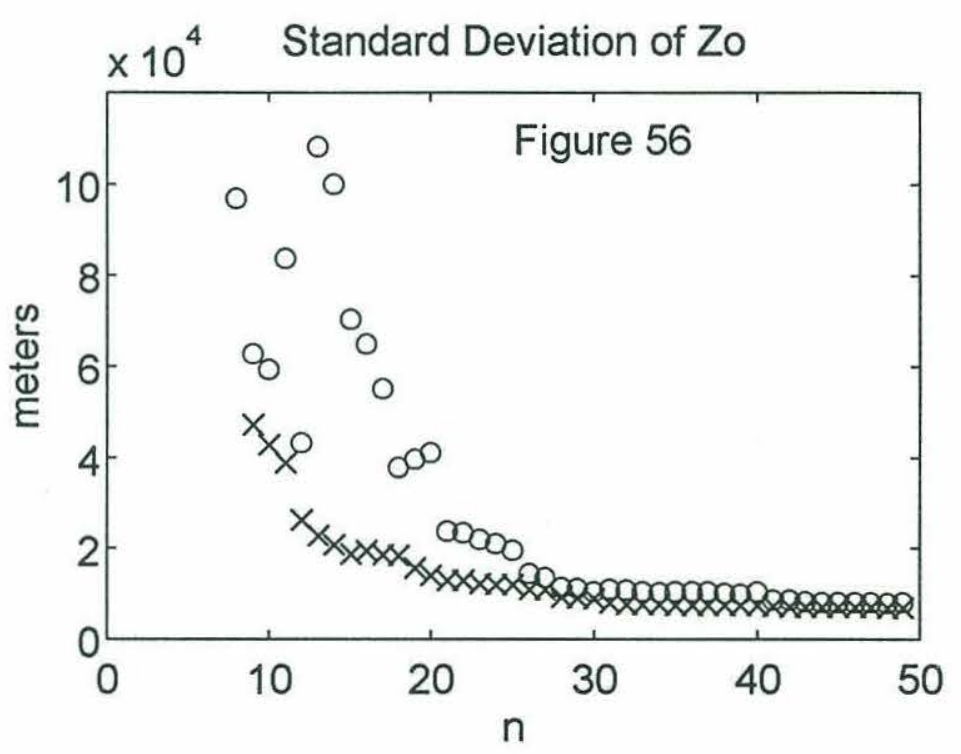

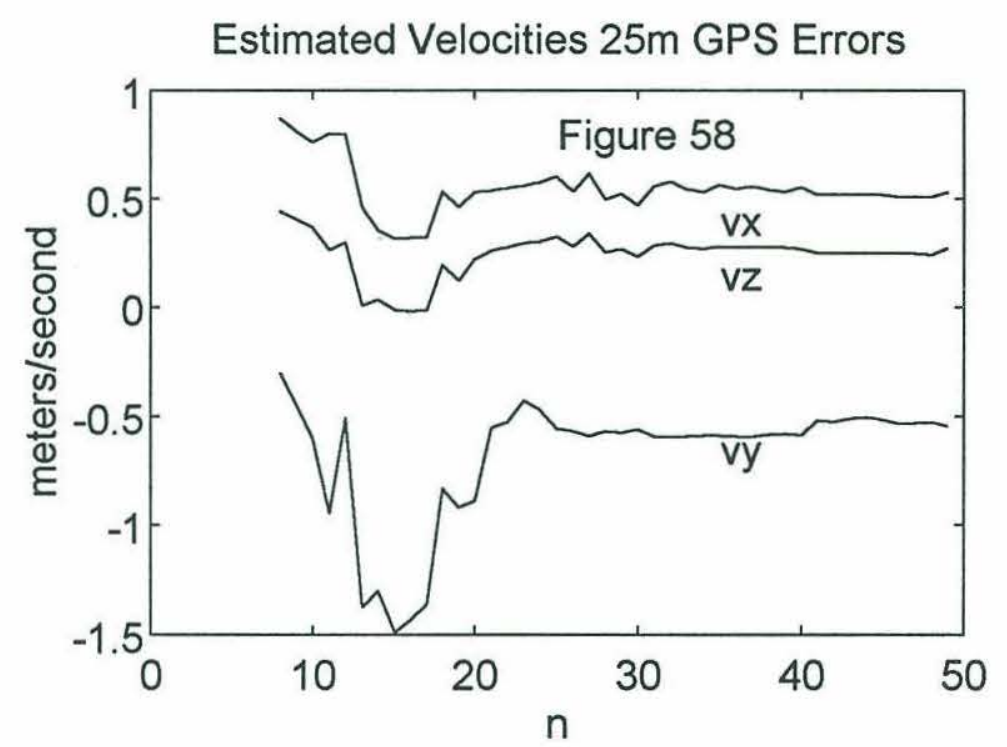


Magnitude of Position Error (Xo,Yo,Zo)

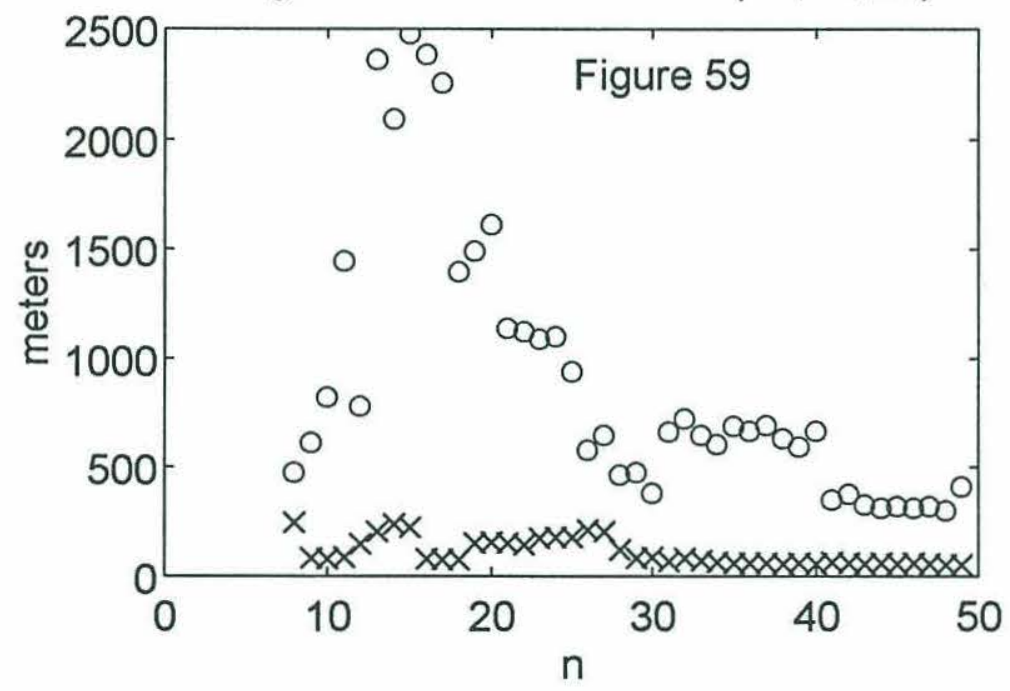

$\infty$

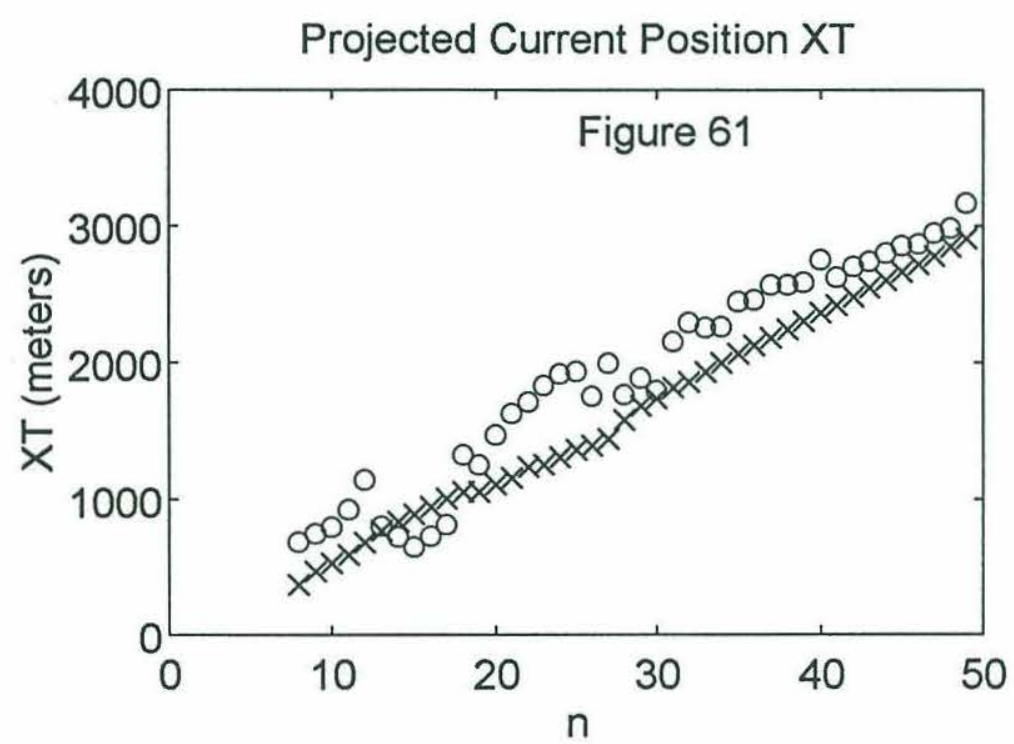

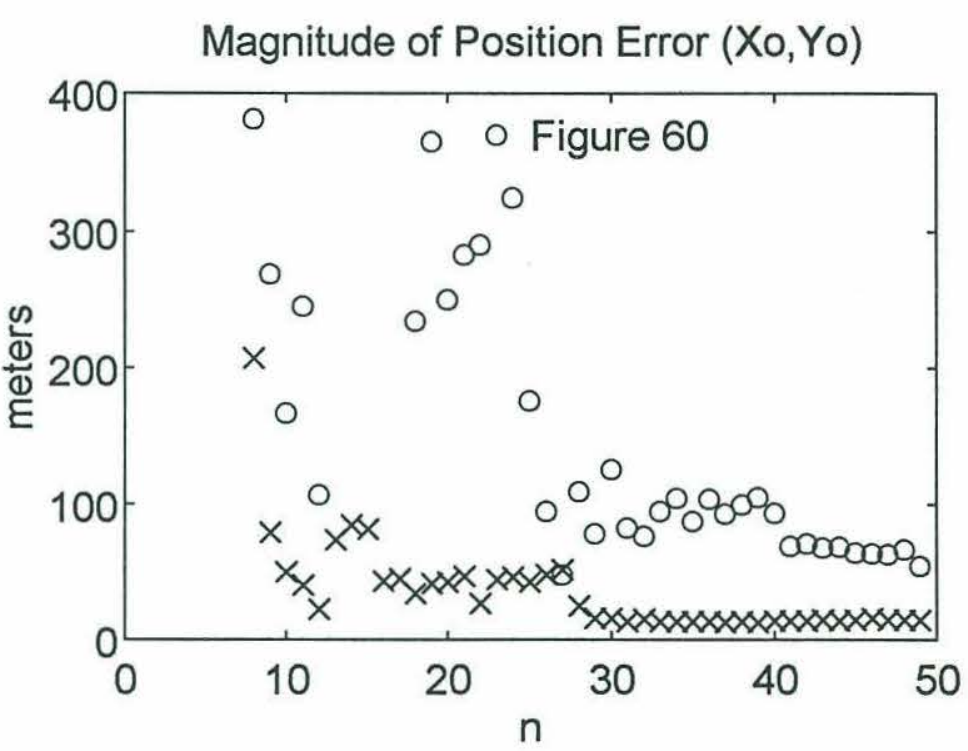

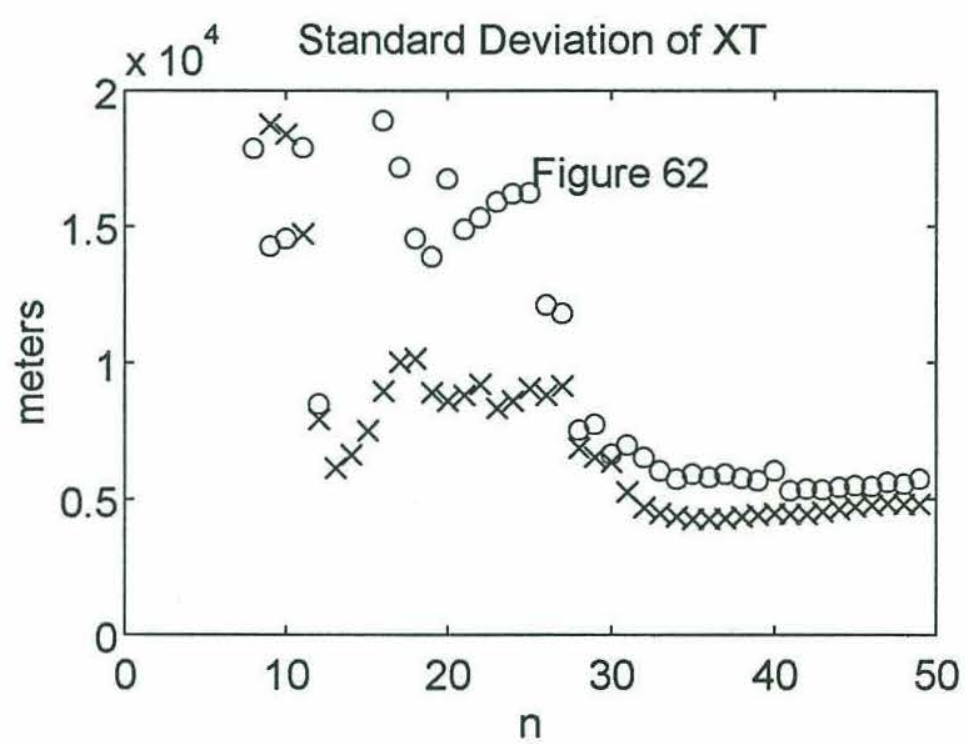




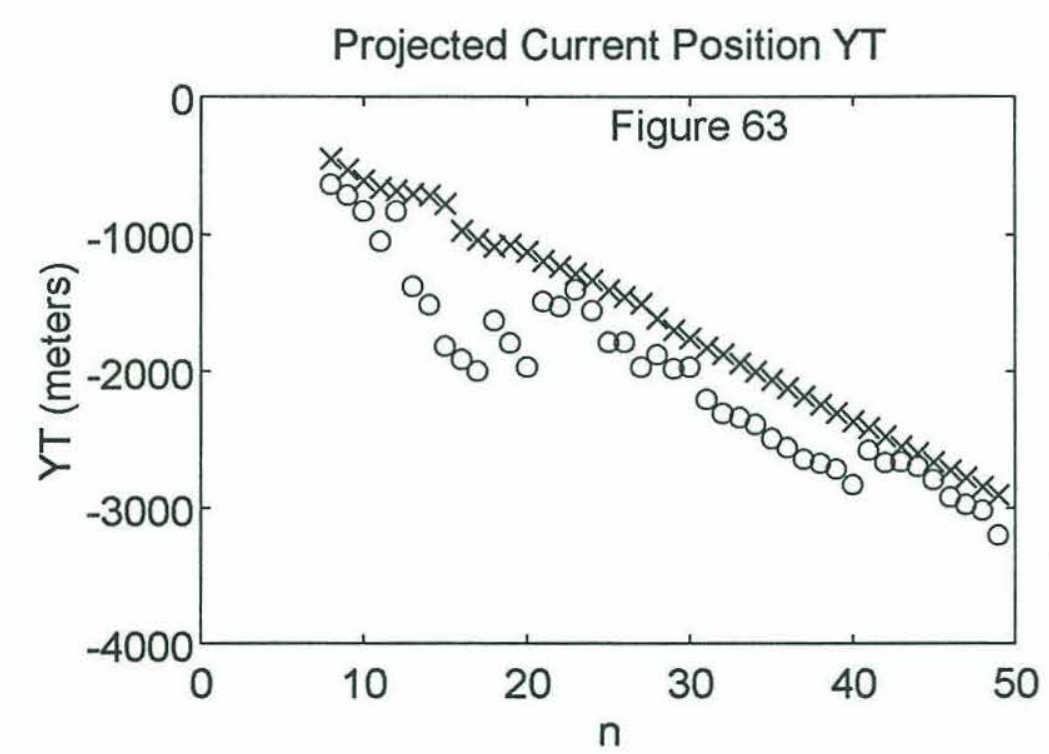

$\stackrel{\infty}{\circ}$

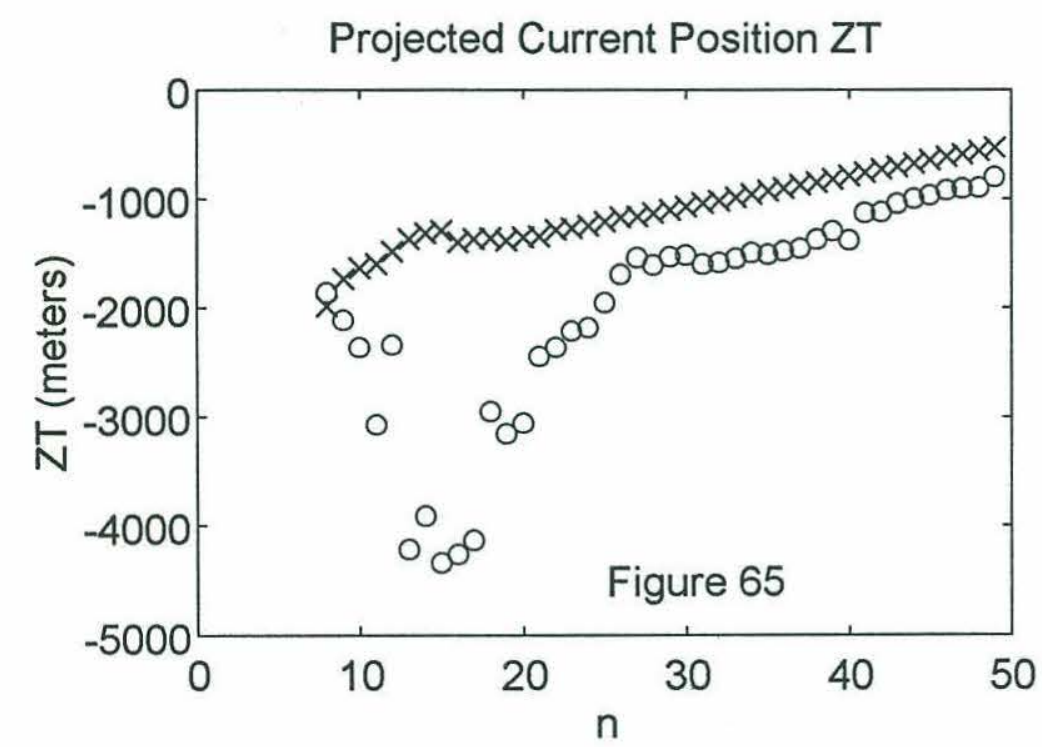

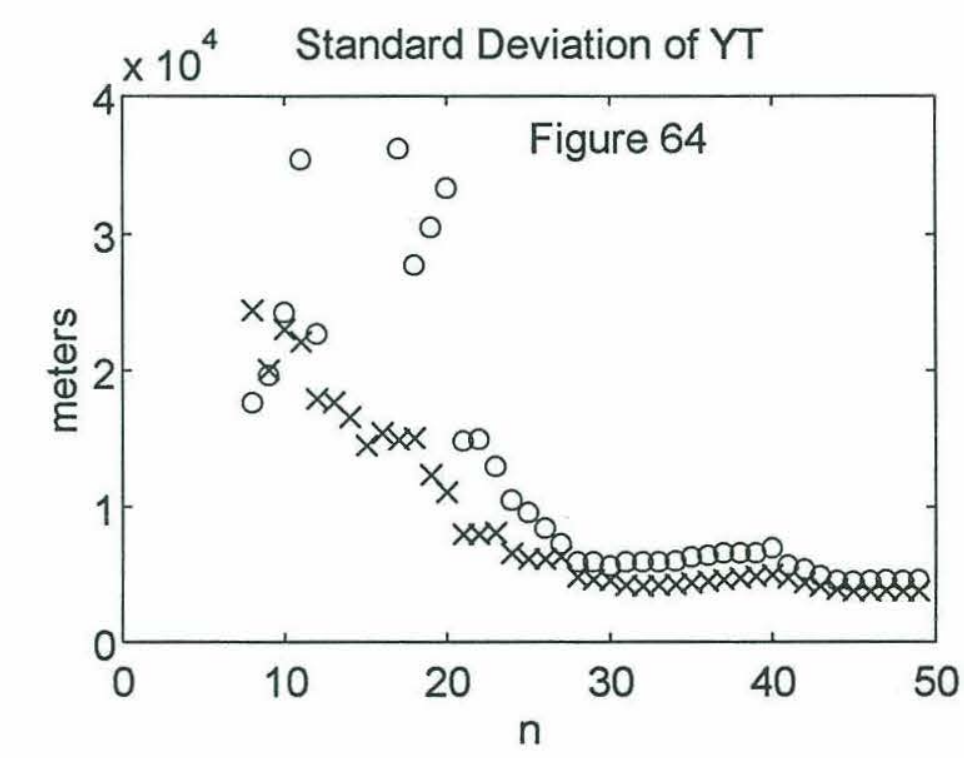

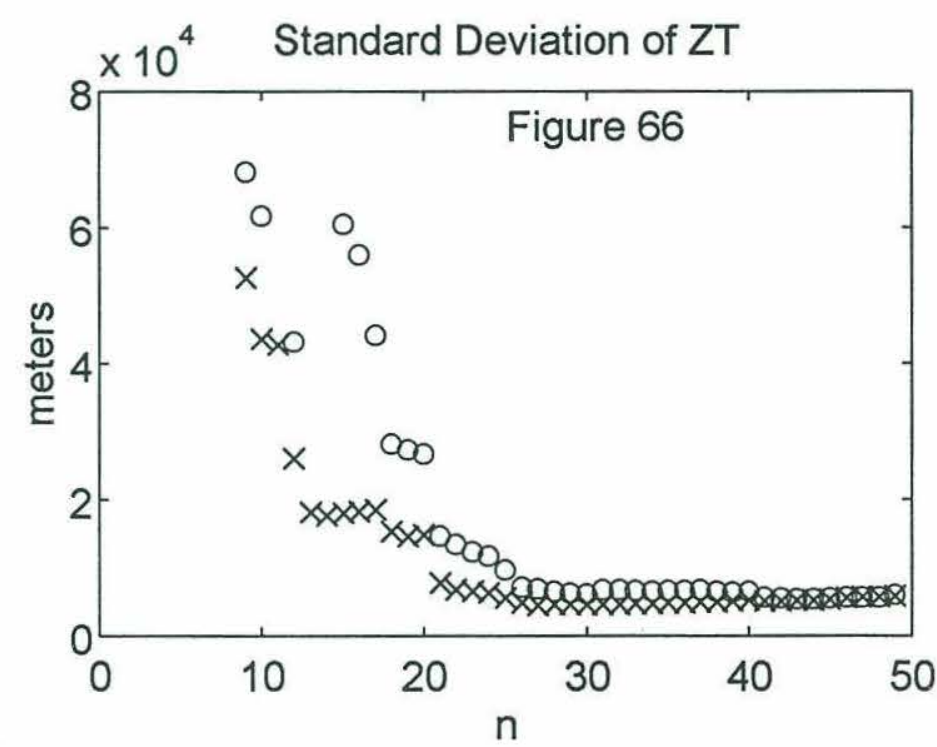



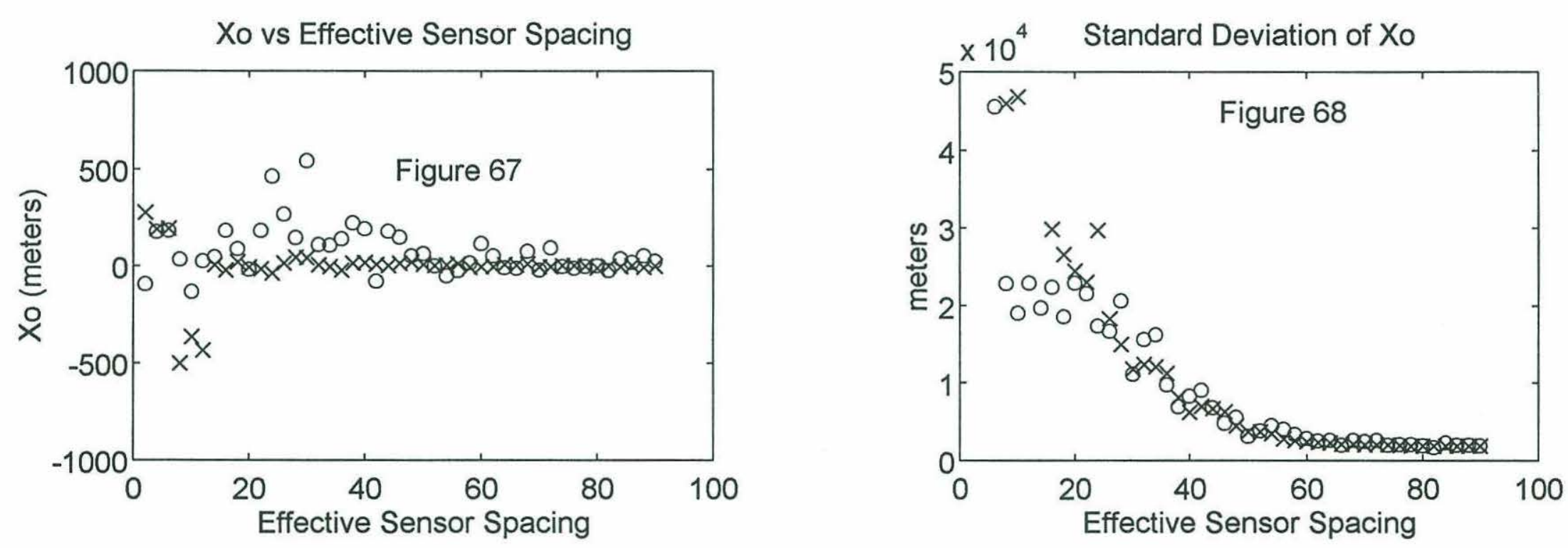

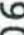
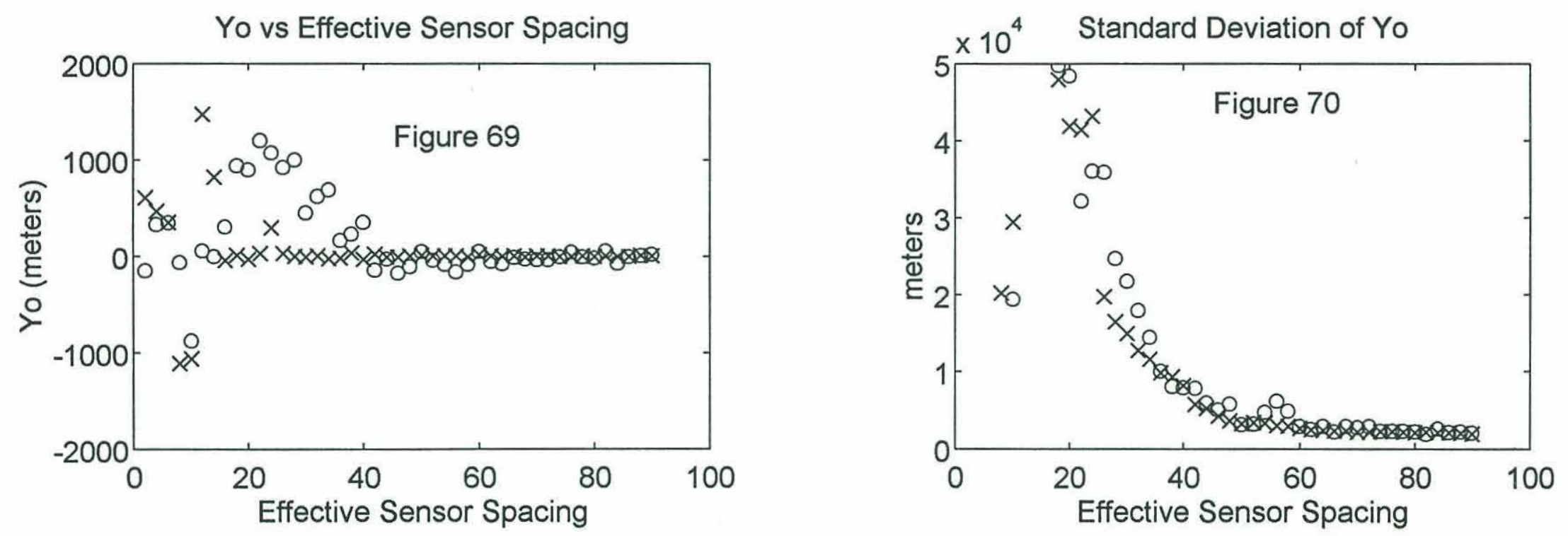

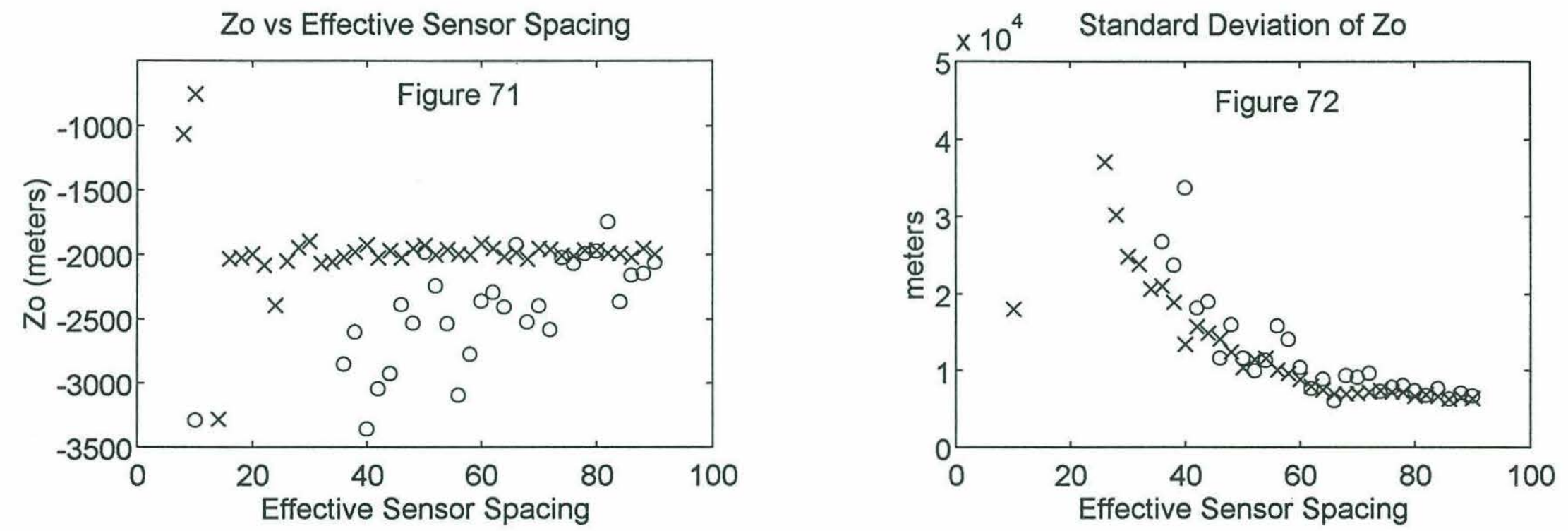

$\stackrel{6}{1}$

Estimated Velocities 3m GPS Errors
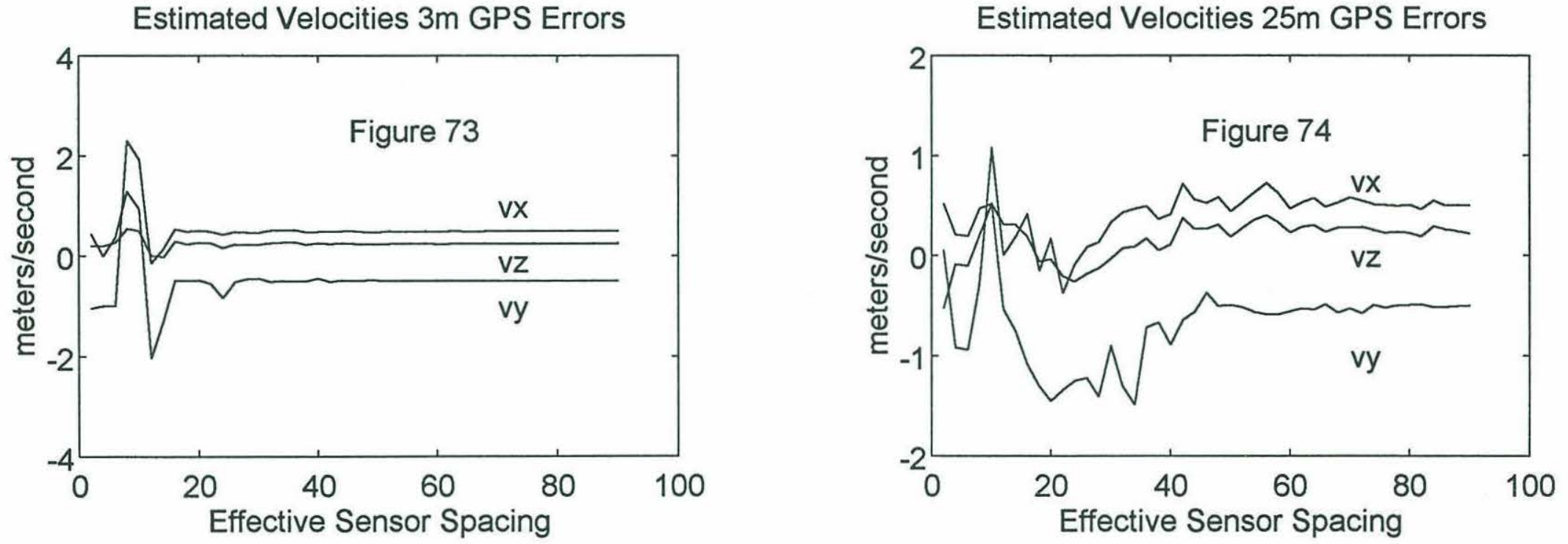
Magnitude of Position Error (Xo, Yo,Zo)

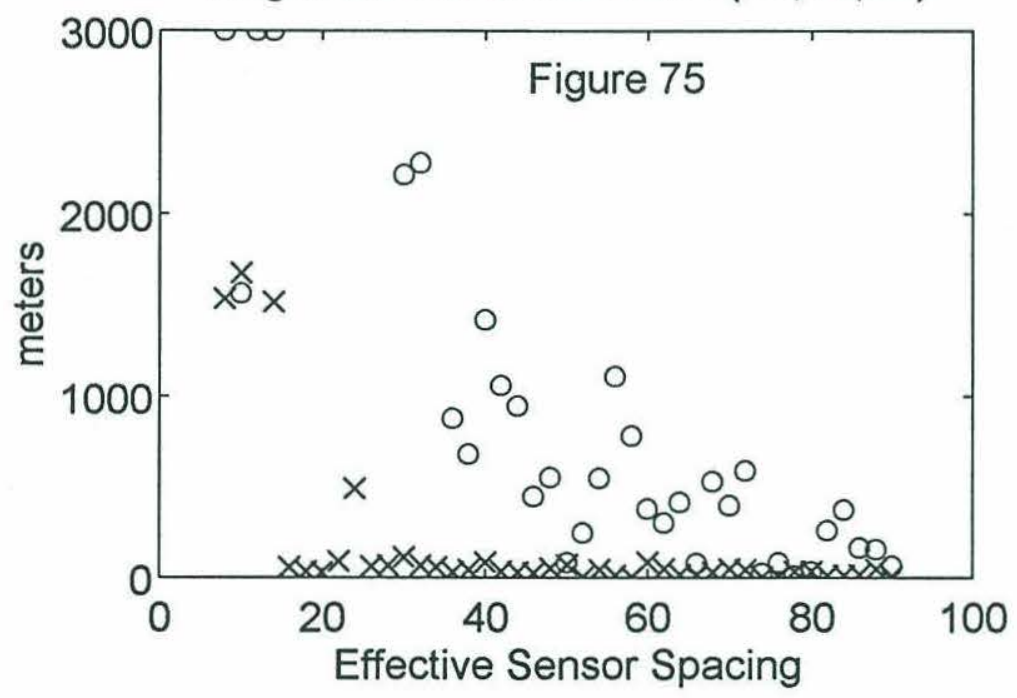

$\stackrel{n}{N}$

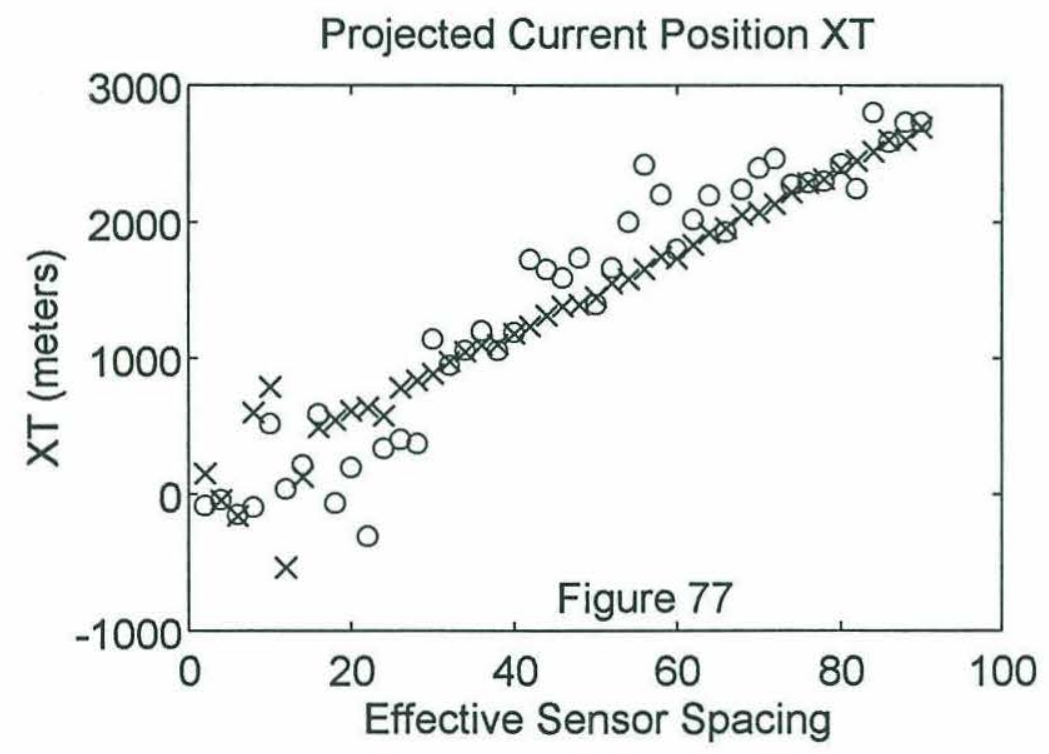

Magnitude of Position Error (Xo,Yo)
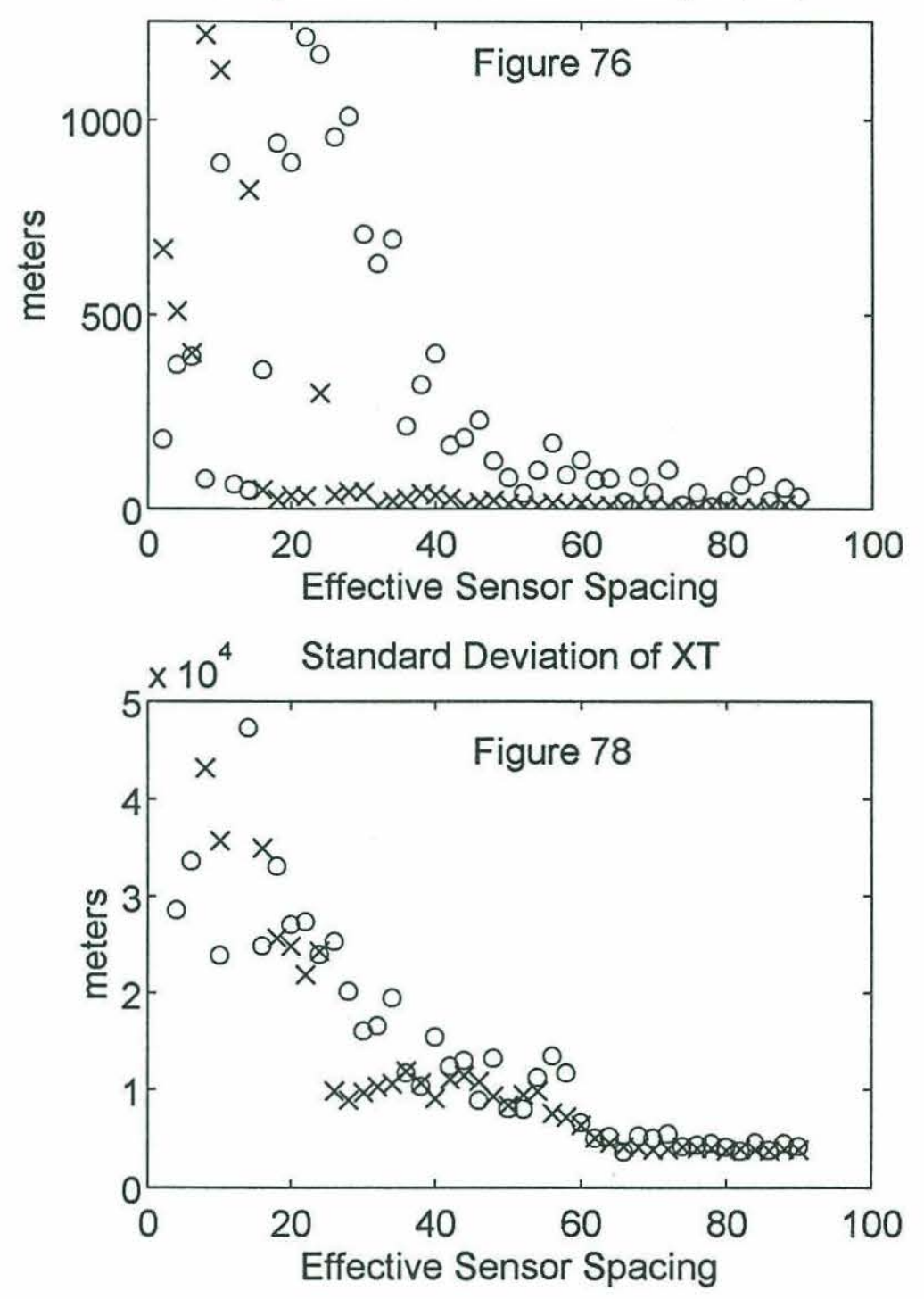

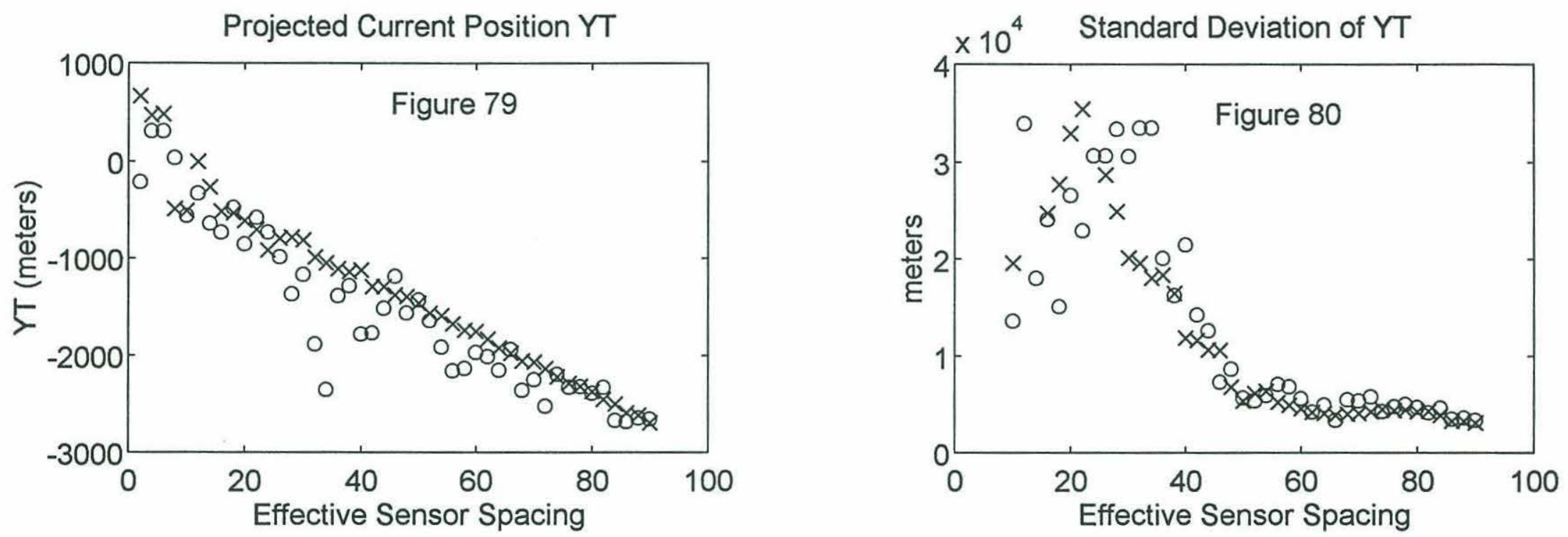

$\stackrel{\circ}{\omega}$
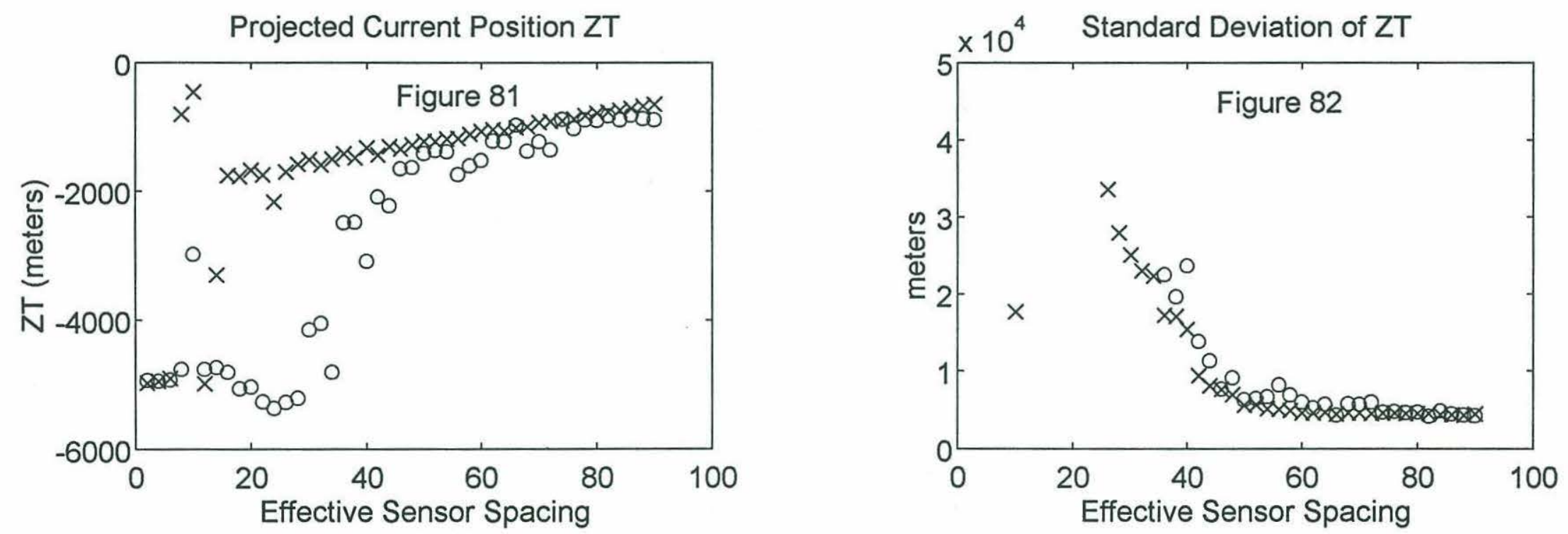


\subsubsection{Scenario 5 - Moving Acoustic Beacon Located at:}

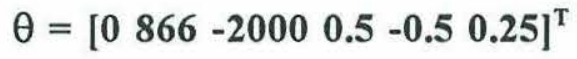

Figures 4-83 through 4-98 show the results of the maximum likelihood estimation algorithm for different numbers of experiment vectors, and figures 4-99 through 4-114 show the results of the maximum likelihood estimation algorithm for different effective sensor spacings for scenario 5. Similar to the previous scenarios, we see that as the number of experiment vectors used in the maximum likelihood estimation problem increases, the accuracy of the estimated position and velocities of the acoustic beacon improves, while the standard deviation of the estimated position and velocities decrease. We also see that as the effective sensor spacing increases, the accuracy of the estimated position and velocities of the acoustic beacon improves, and the standard deviation of the estimated position decreases. As before, the position and velocity errors initially decrease rapidly, however after the number of experiment vectors used reaches approximately 30 , and the effective sensor spacing reaches approximately 45 , the errors and the standard deviations decrease much slower.

Figures 4-93 through 4-98 and figures 4-109 through 4-114 show the estimation algorithm's ability to track the linearly moving acoustic beacon for this scenario. We see that the projected current positions follow the linearly moving acoustic beacon fairly well for both 3 meter and 25 meter GPS errors. This is because the estimated initial positions $\mathrm{x}_{\mathrm{o}}, \mathrm{y}_{\mathrm{o}}$, and $\mathrm{z}_{\mathrm{o}}$ obtained using 25 meter GPS errors were better in this scenario then for scenario 4 . In general this is not the case, however the measurement errors in this scenario simply led to a better estimate of the acoustic beacon's position. 

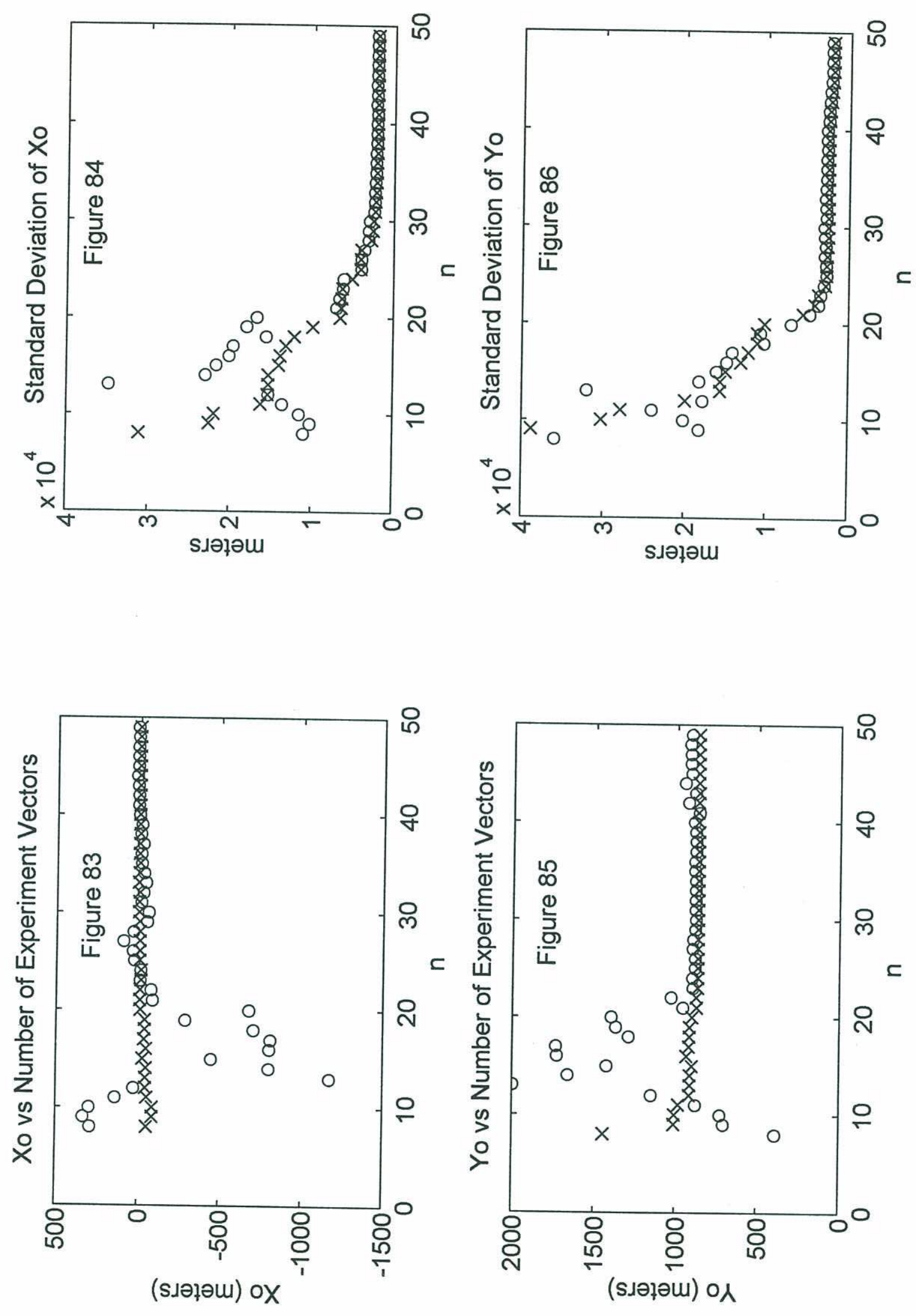

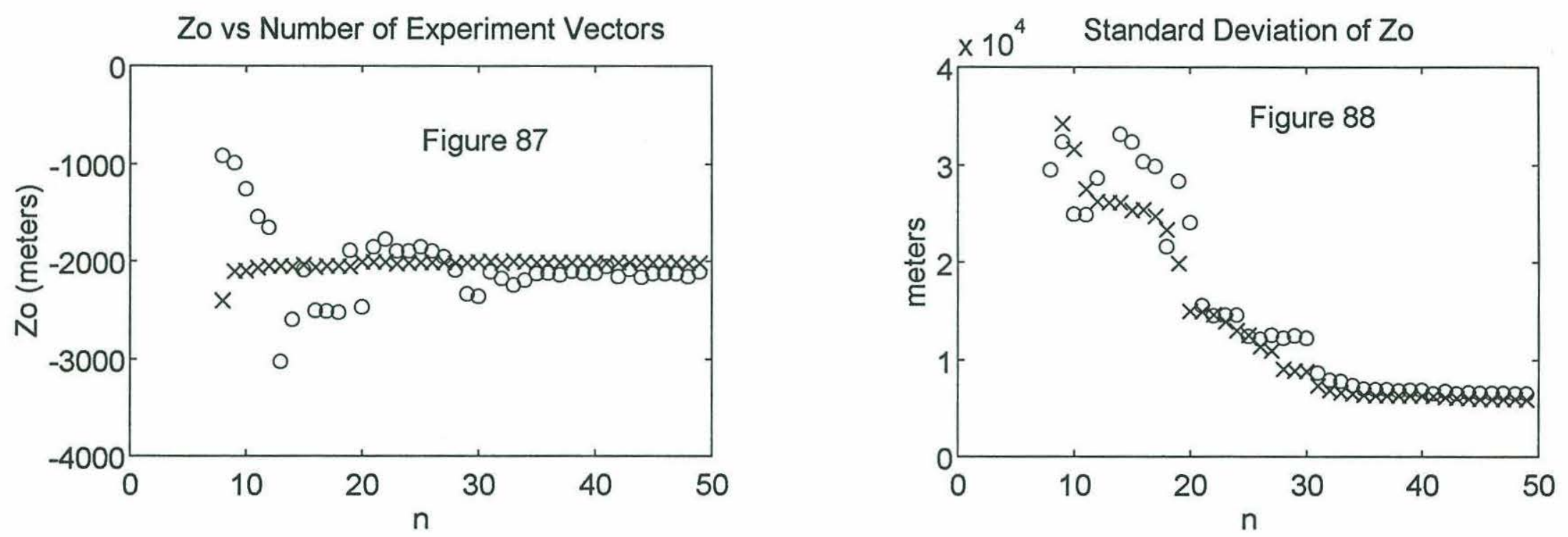

ดั
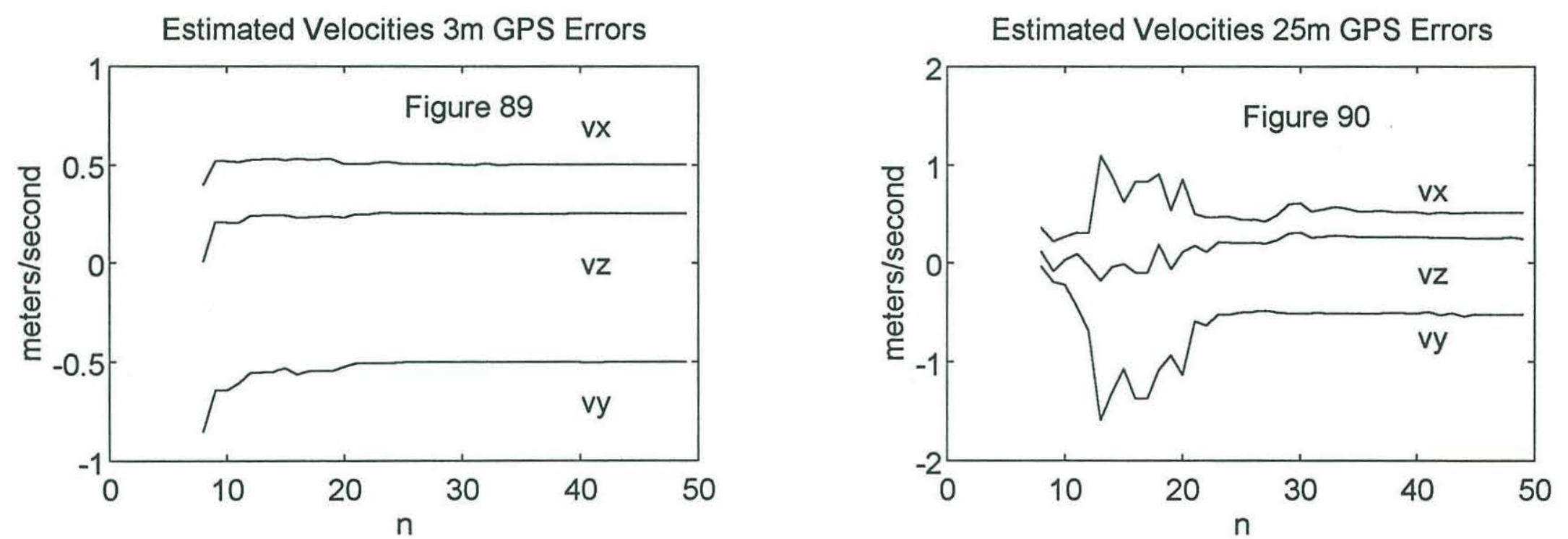

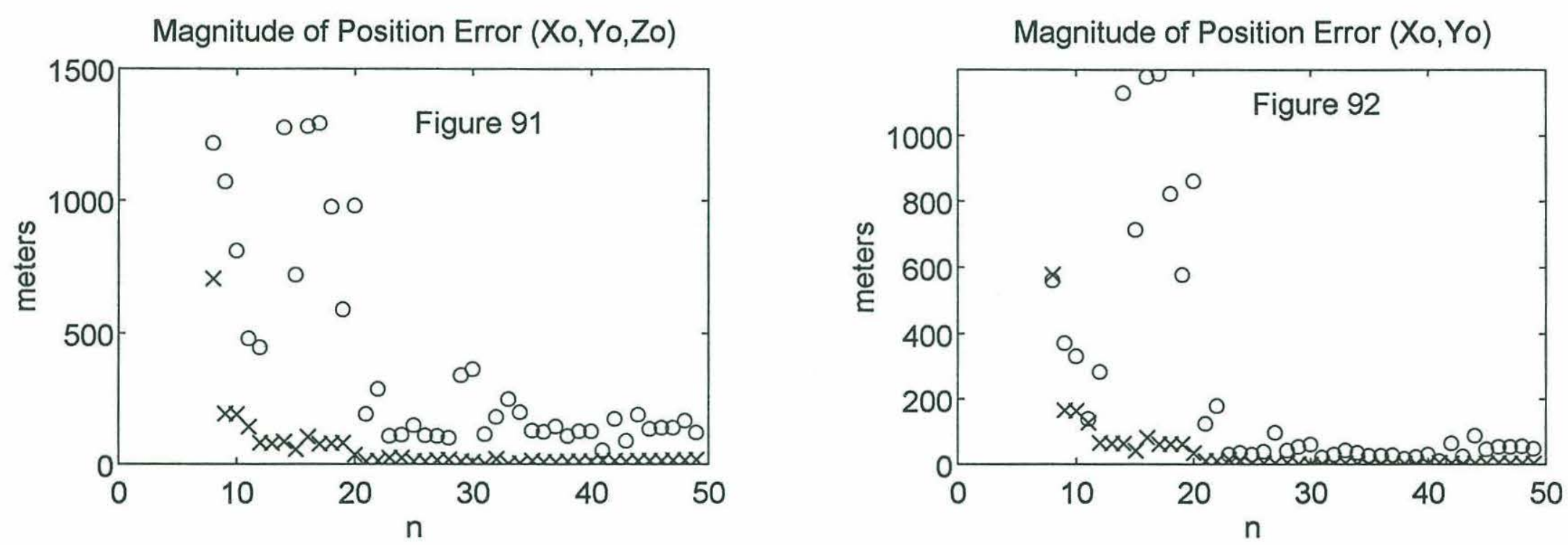

$\stackrel{2}{2}$
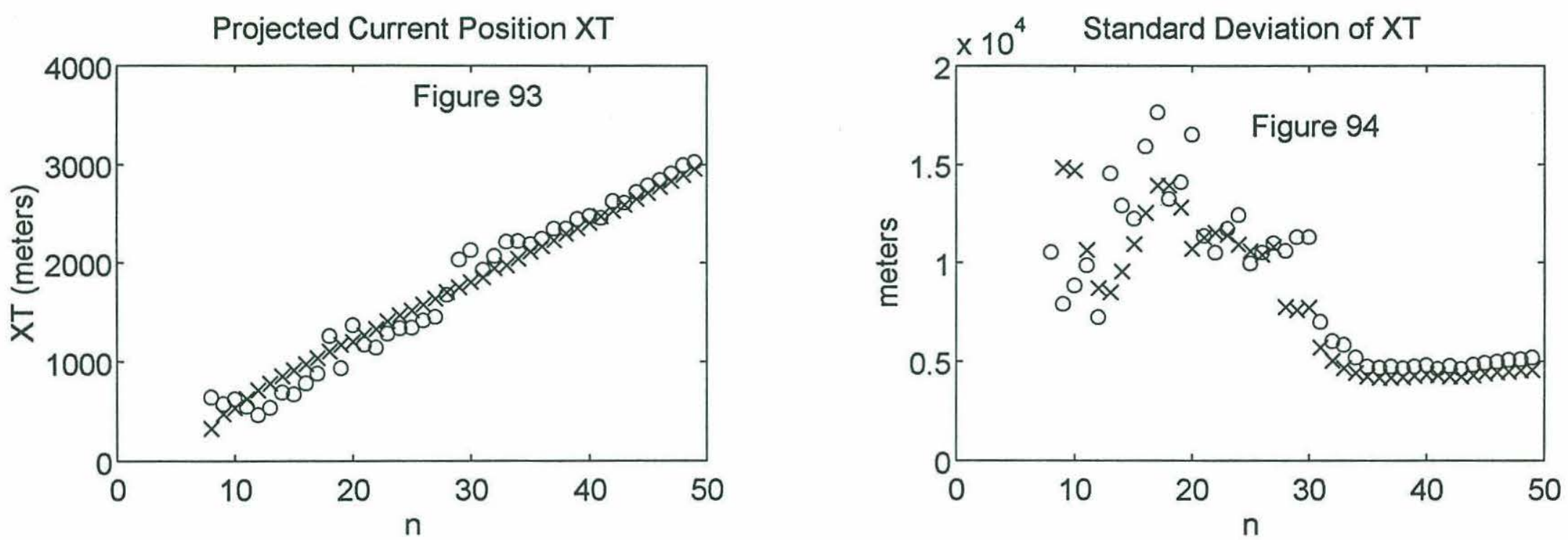

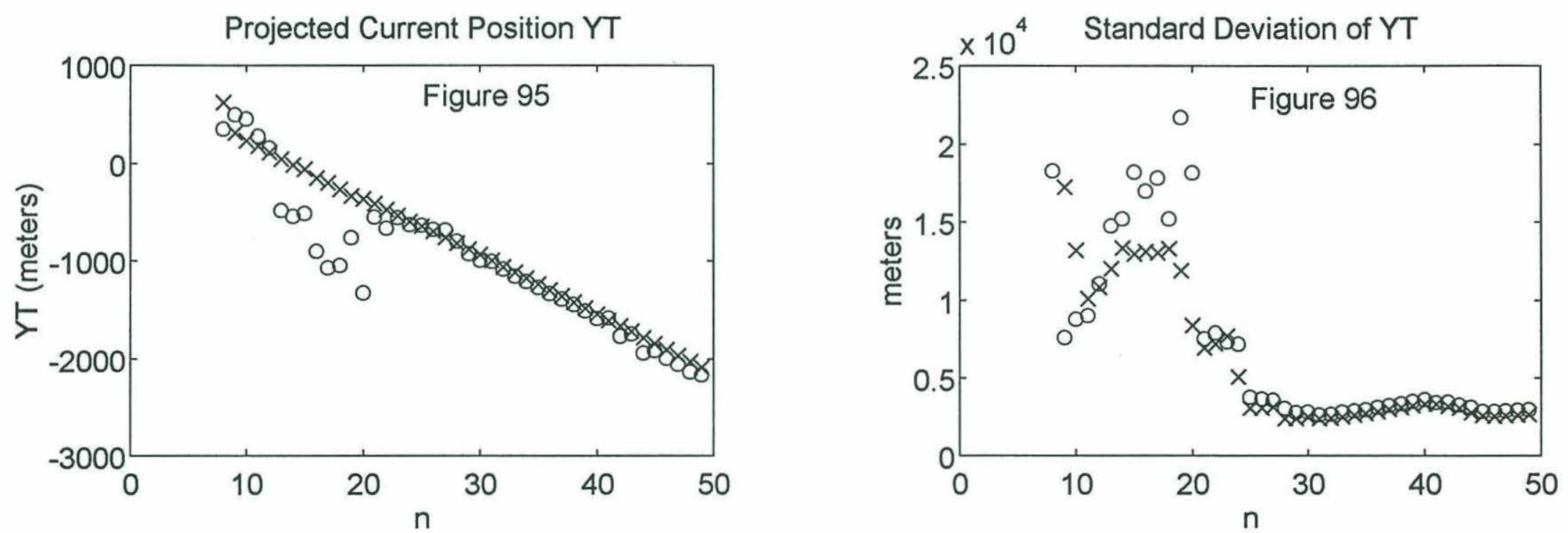

$\underset{\infty}{\infty}$
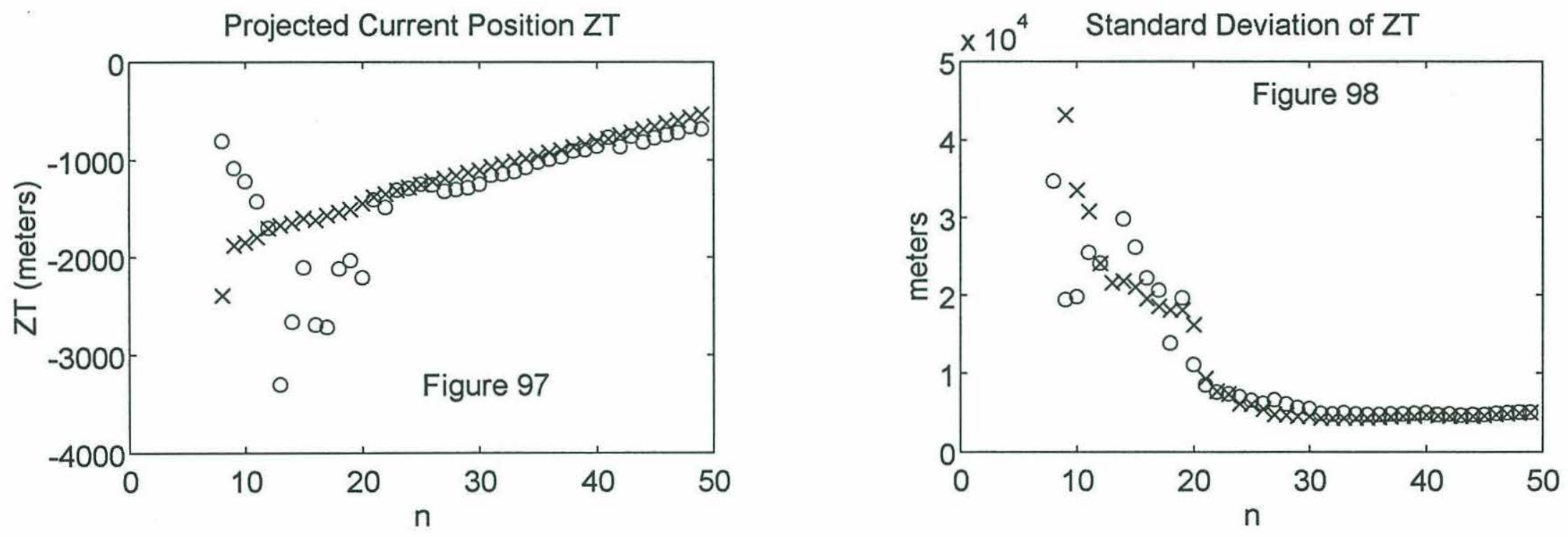

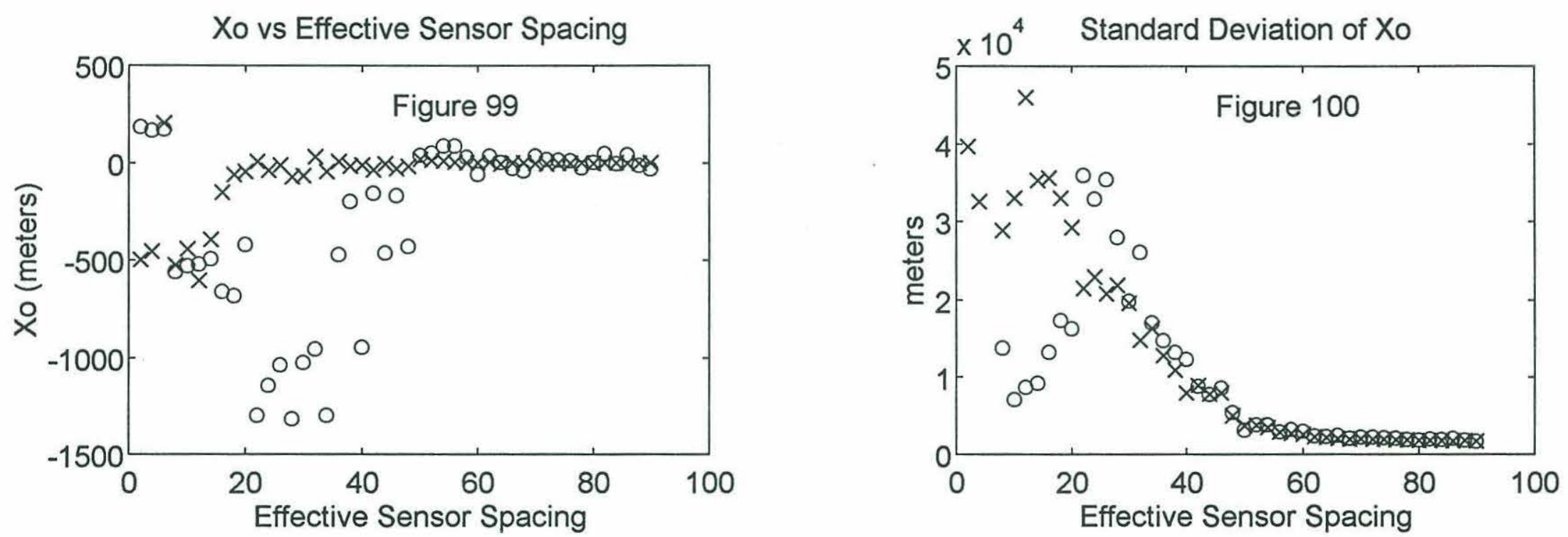

$\stackrel{\circ}{\circ}$
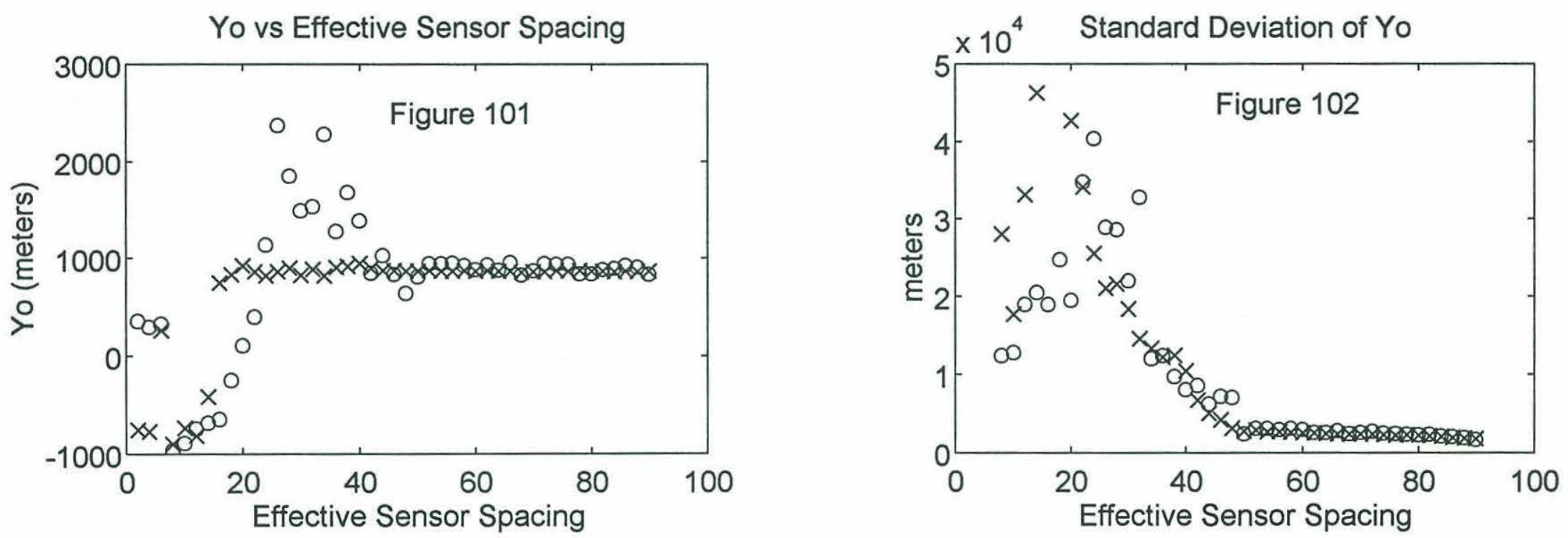

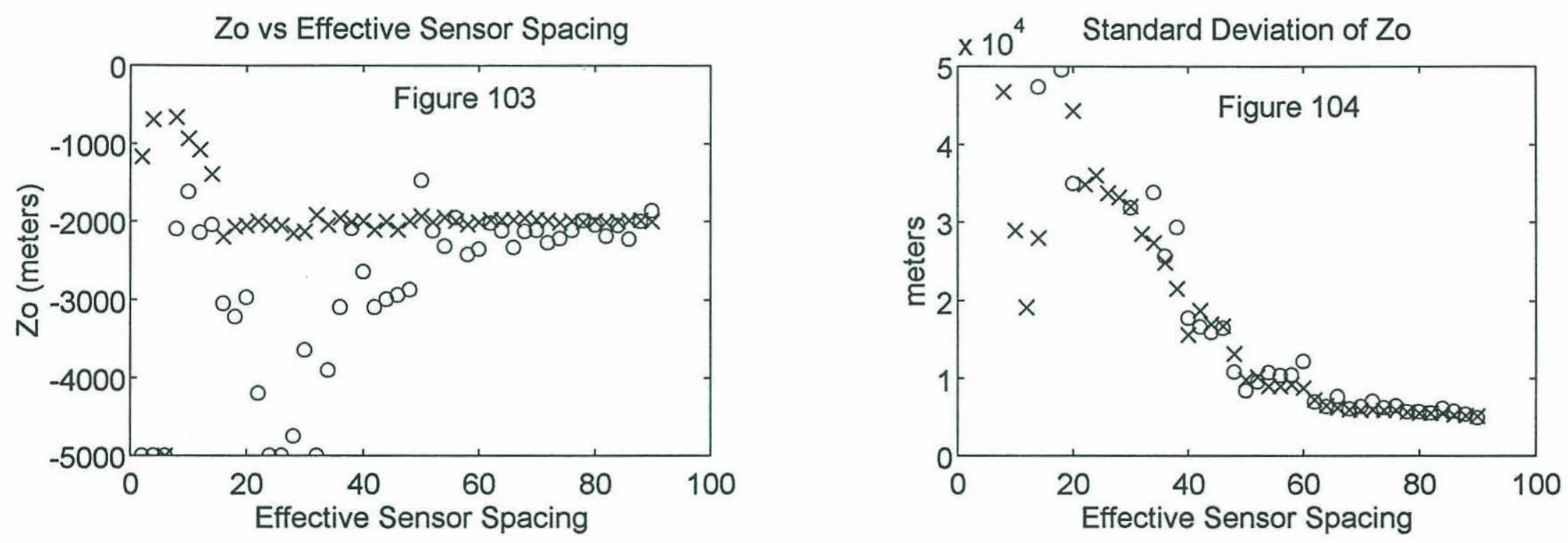

홍
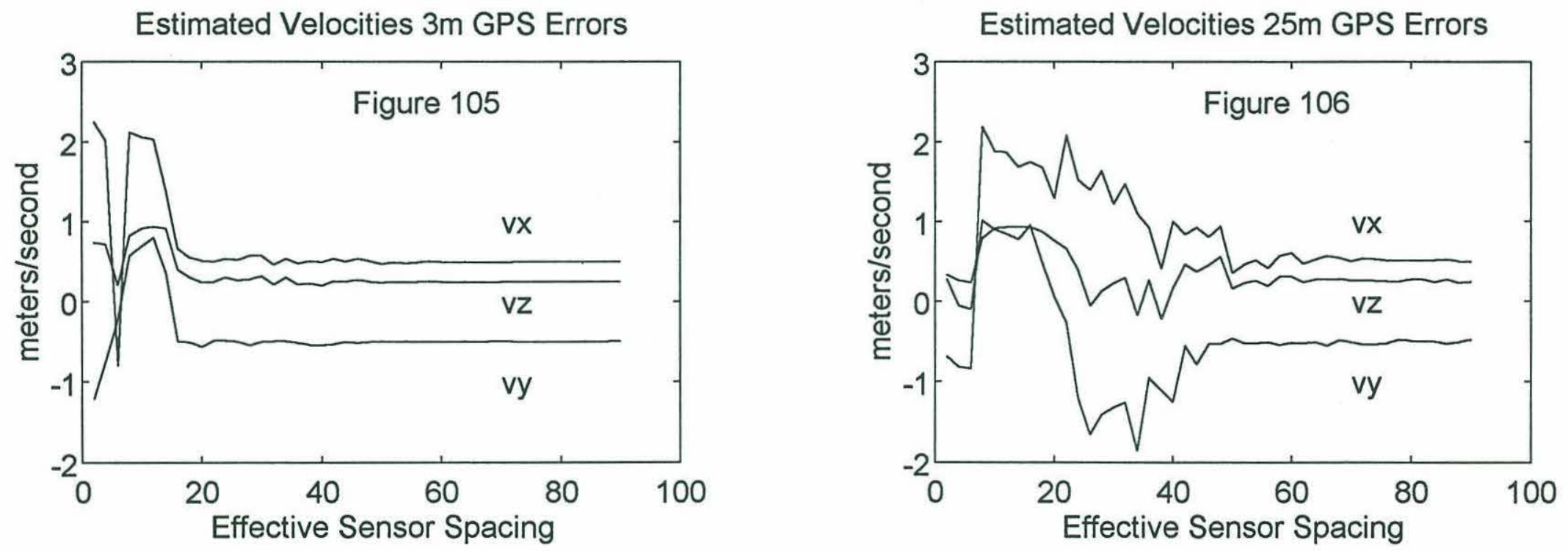

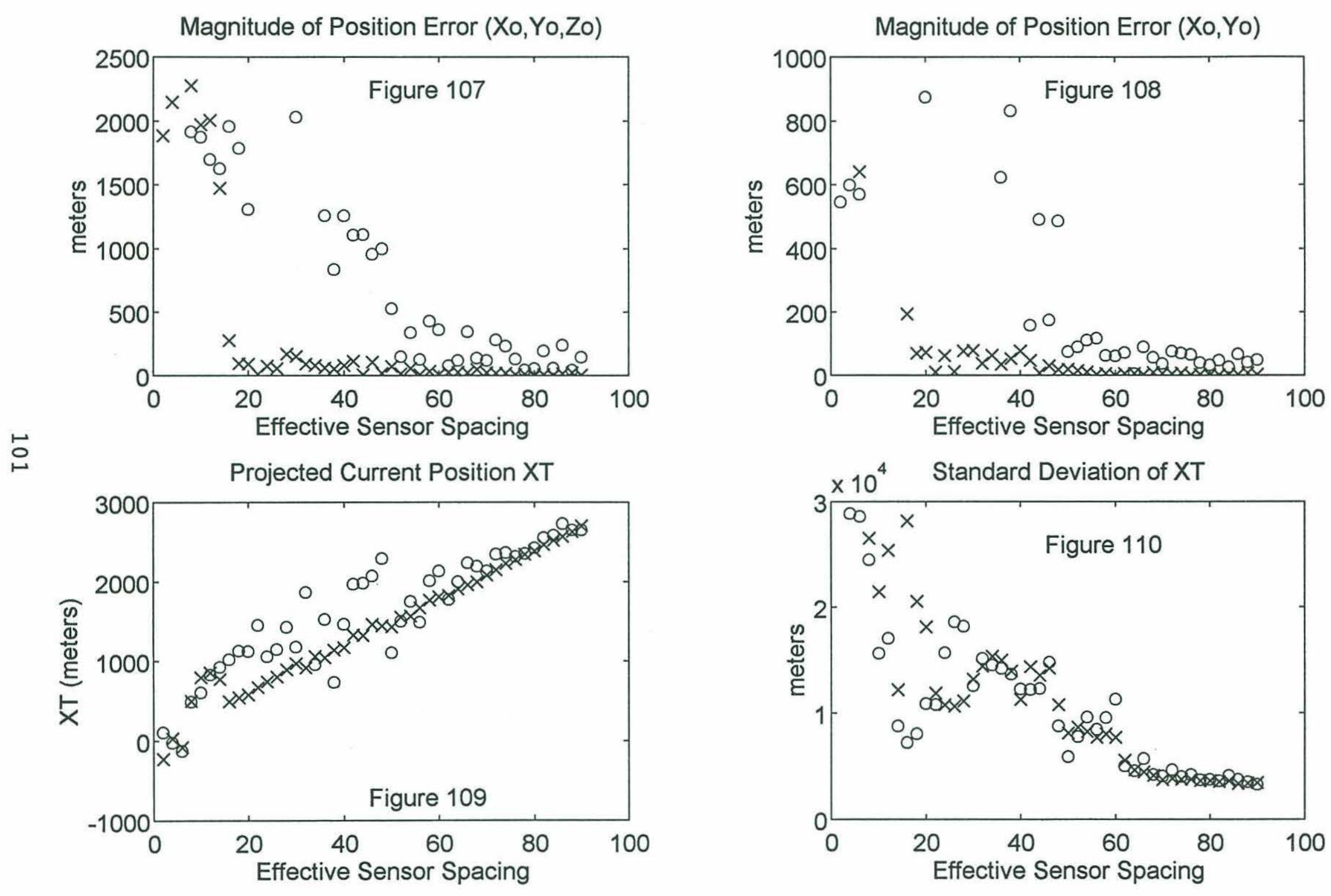

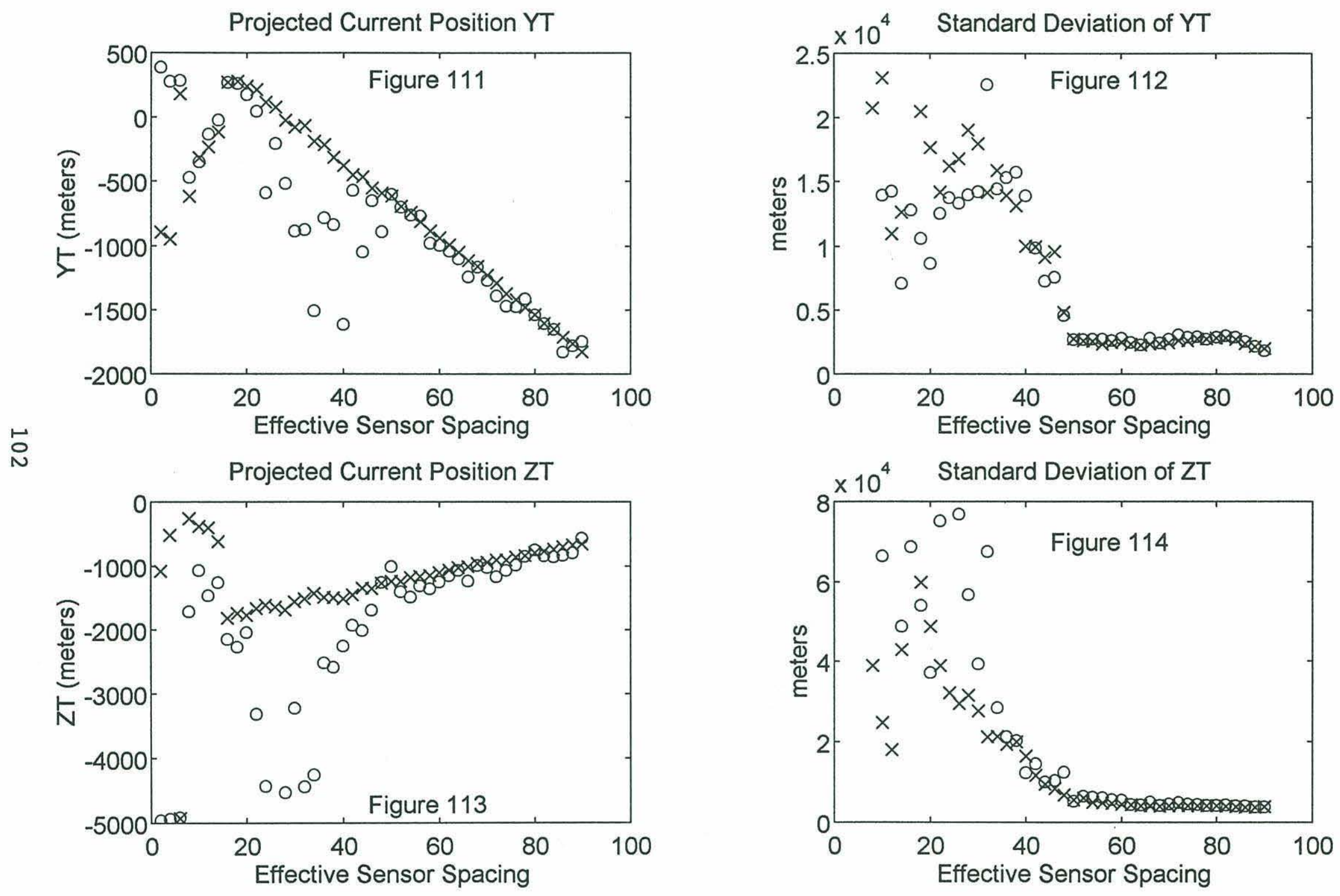


\subsubsection{Scenario 6 - Moving Acoustic Beacon Located at:}

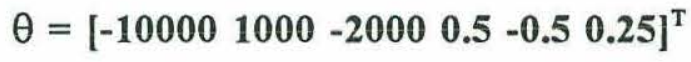

Figures 4-115 through 4-130 show the results of the maximum likelihood estimation algorithm for different numbers of experiment vectors, and figures 4-131 through 4-146 show the results of the maximum likelihood estimation algorithm for different effective sensor spacings for scenario 6. Again we see that as the number of experiment vectors used in the maximum likelihood estimation problem increases, the accuracy of the estimated position and velocities of the acoustic beacon improves, and the standard deviation of the estimated position and velocities decrease. We also see that as the effective sensor spacing increases, the accuracy of the estimated position and velocities of the acoustic beacon improves, while the standard deviation of the estimated position decreases. As before, the position and velocity errors initially decrease rapidly, however after the number of experiment vectors used reaches approximately 30 , or the effective sensor spacing reaches approximately 45 , the errors and the standard deviations decrease much slower.

Figures 4-125 through 4-130 and figures 4-141 through 4-146 show the estimation algorithm's ability to track the linearly moving acoustic beacon for this scenario. We see that the projected current positions follow the linearly moving acoustic beacon fairly well using 3 meter GPS errors. Again, because of the errors in estimating the acoustic beacon's initial positions $x_{0}, y_{0}$, and $z_{0}$, the projected current position obtained from using 25 meter GPS errors does not track the acoustic beacon's motion as well. 

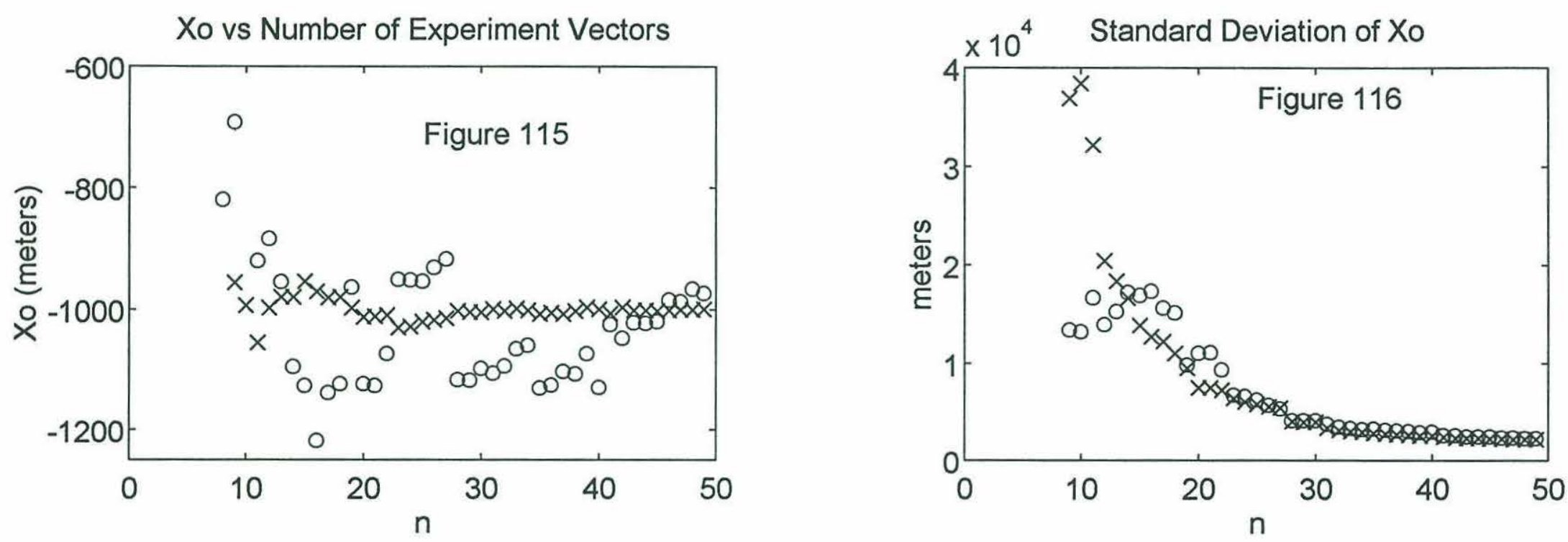

占
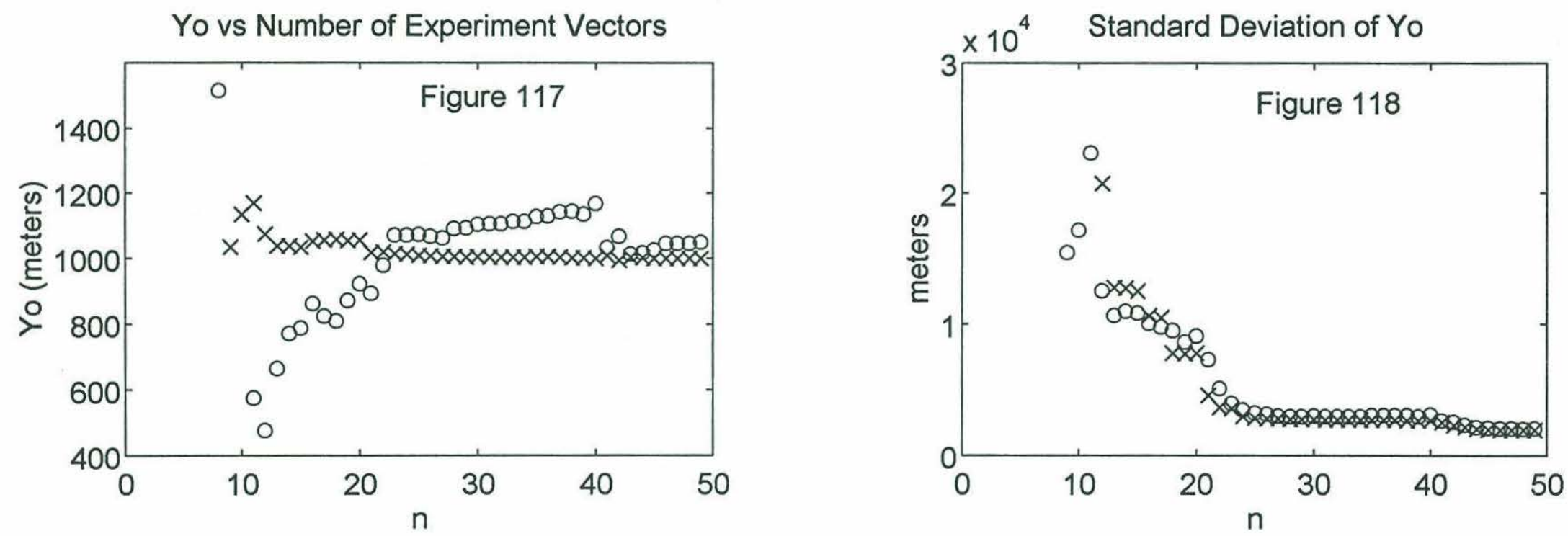
Zo vs Number of Experiment Vectors

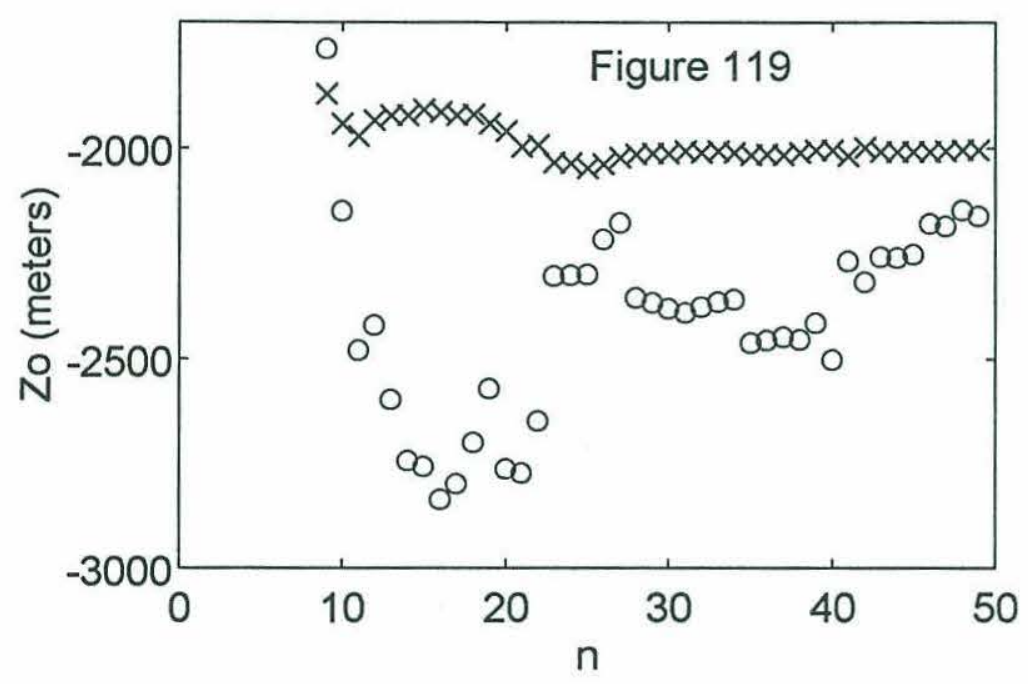

웅

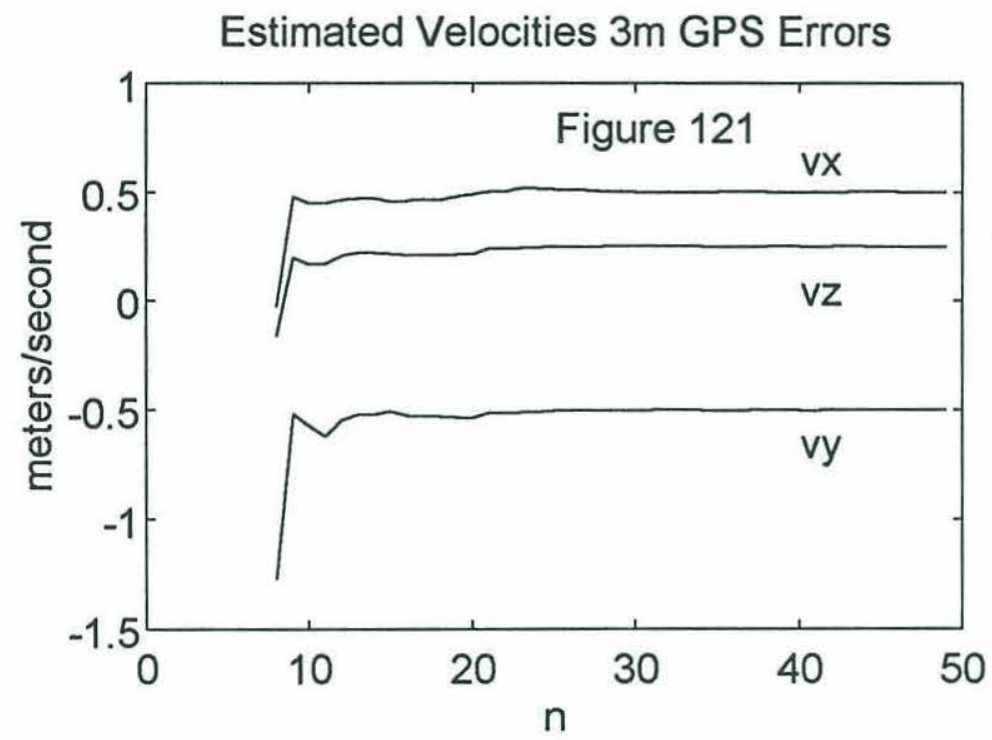

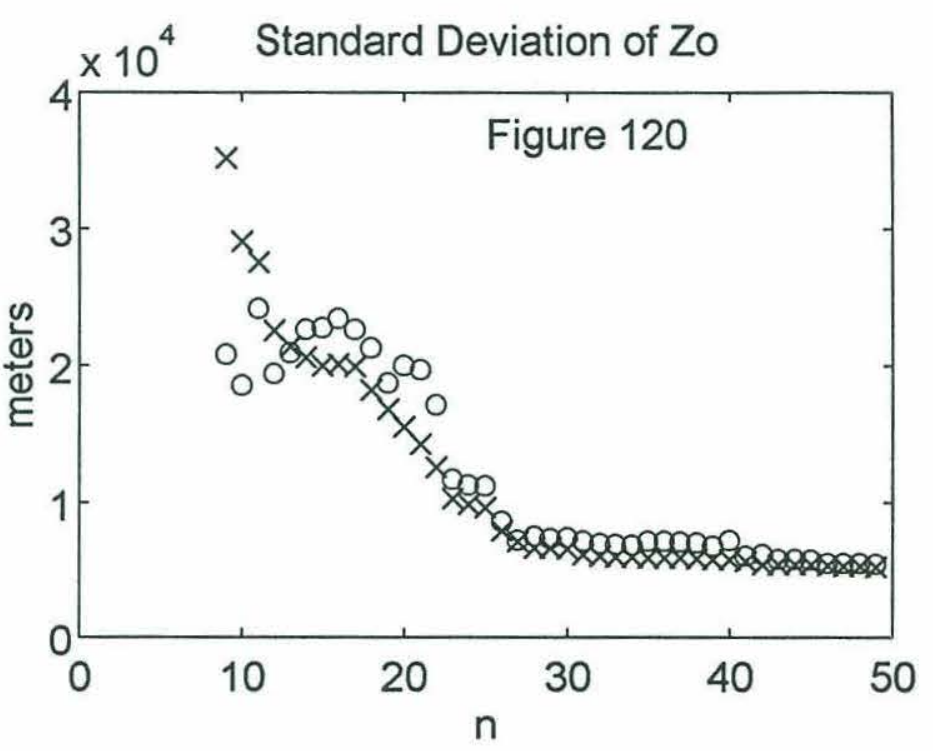

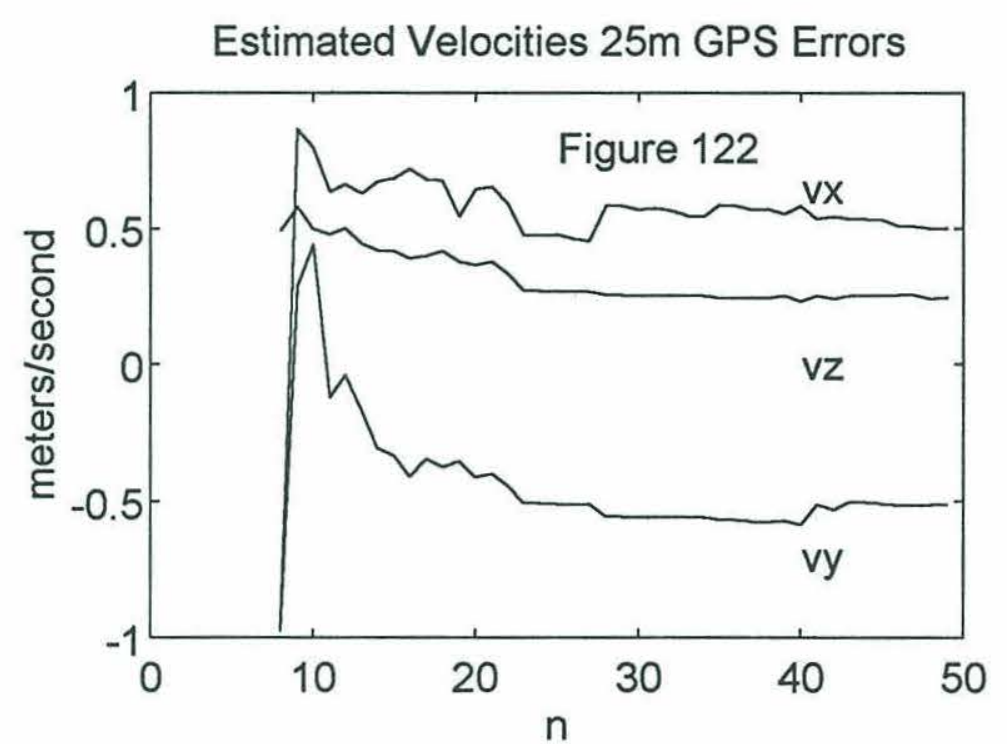



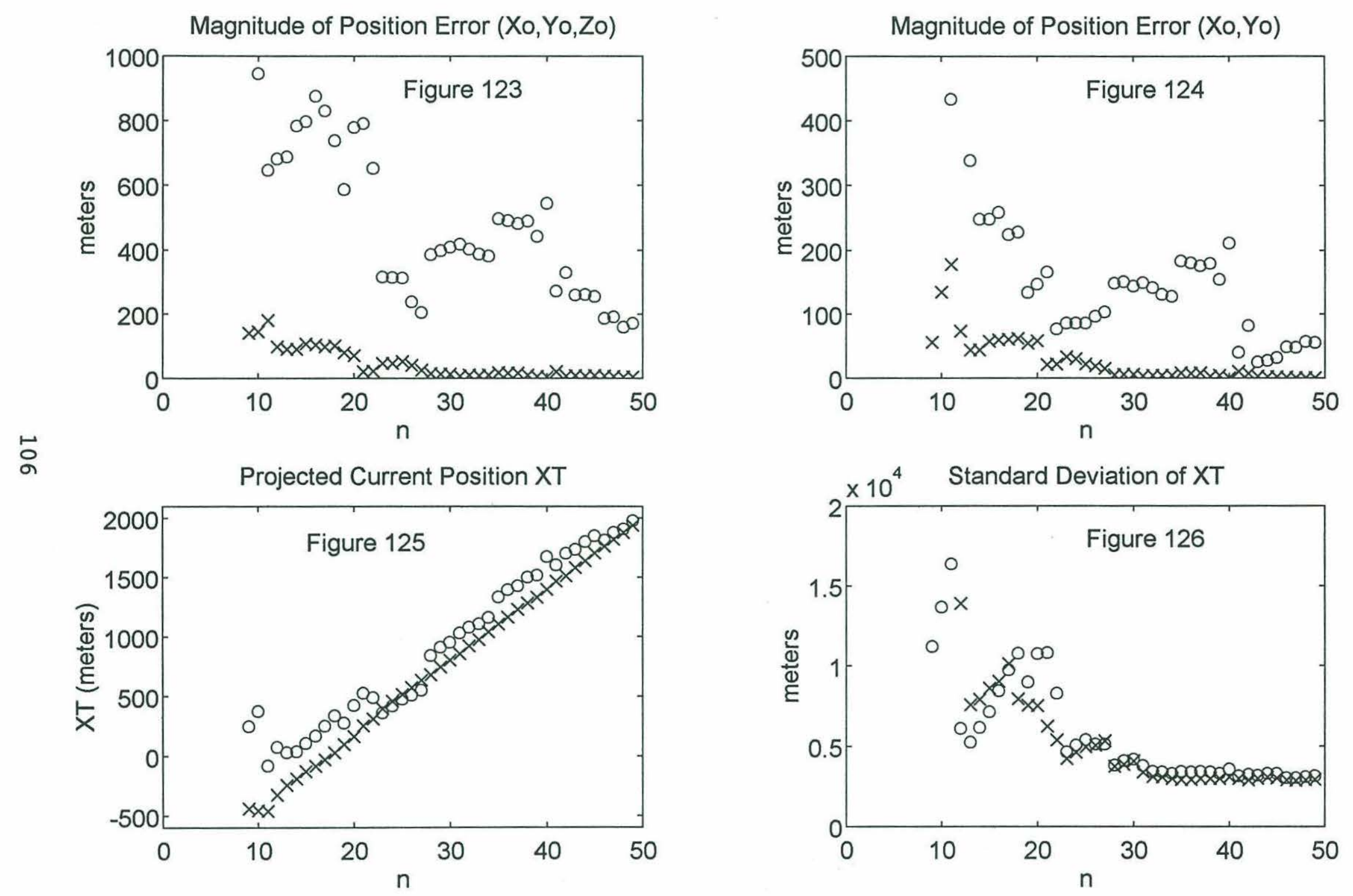

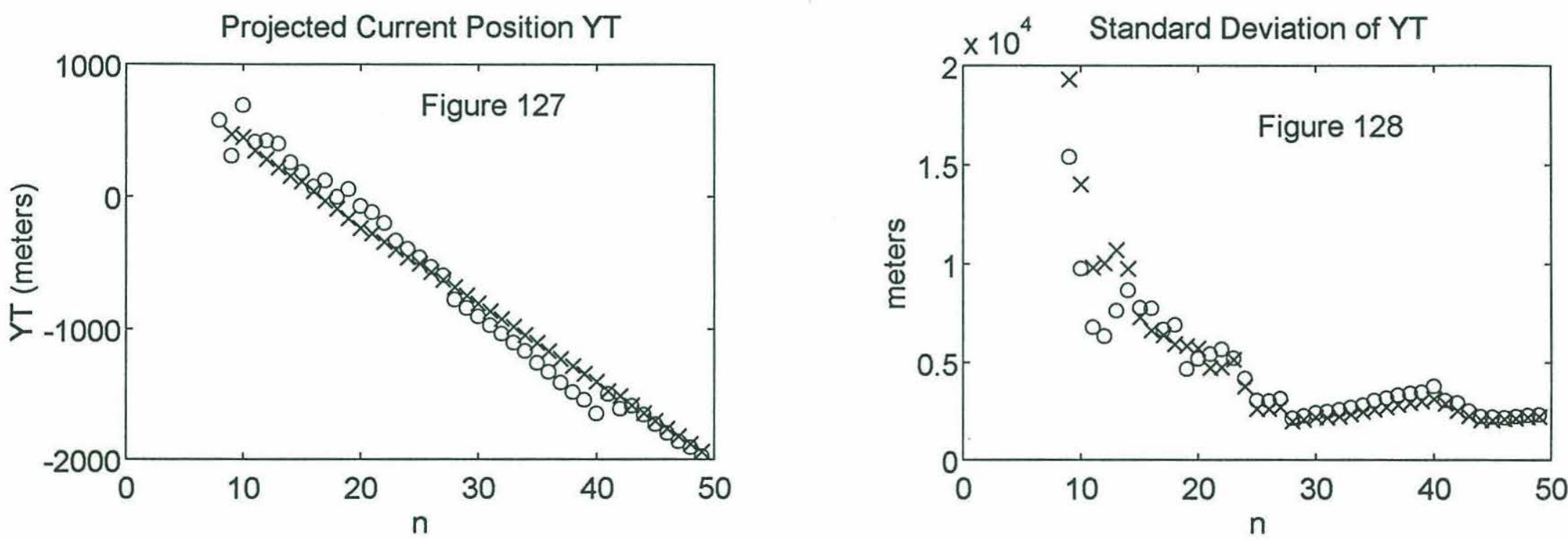

옵
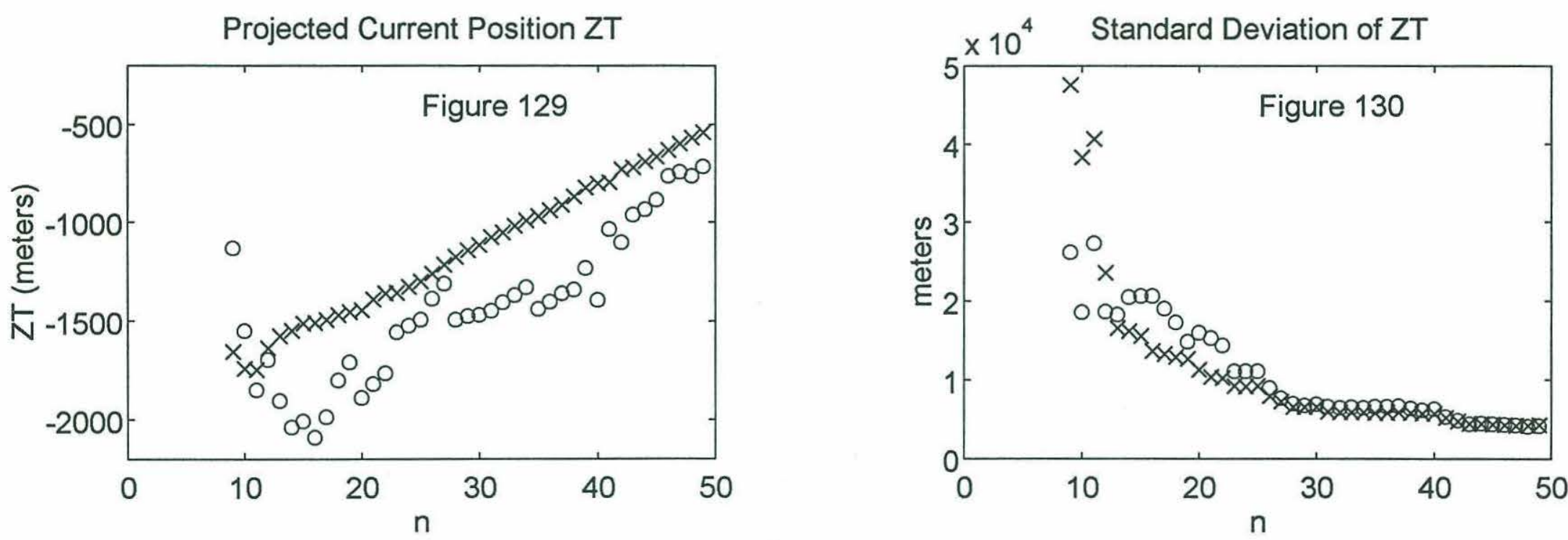

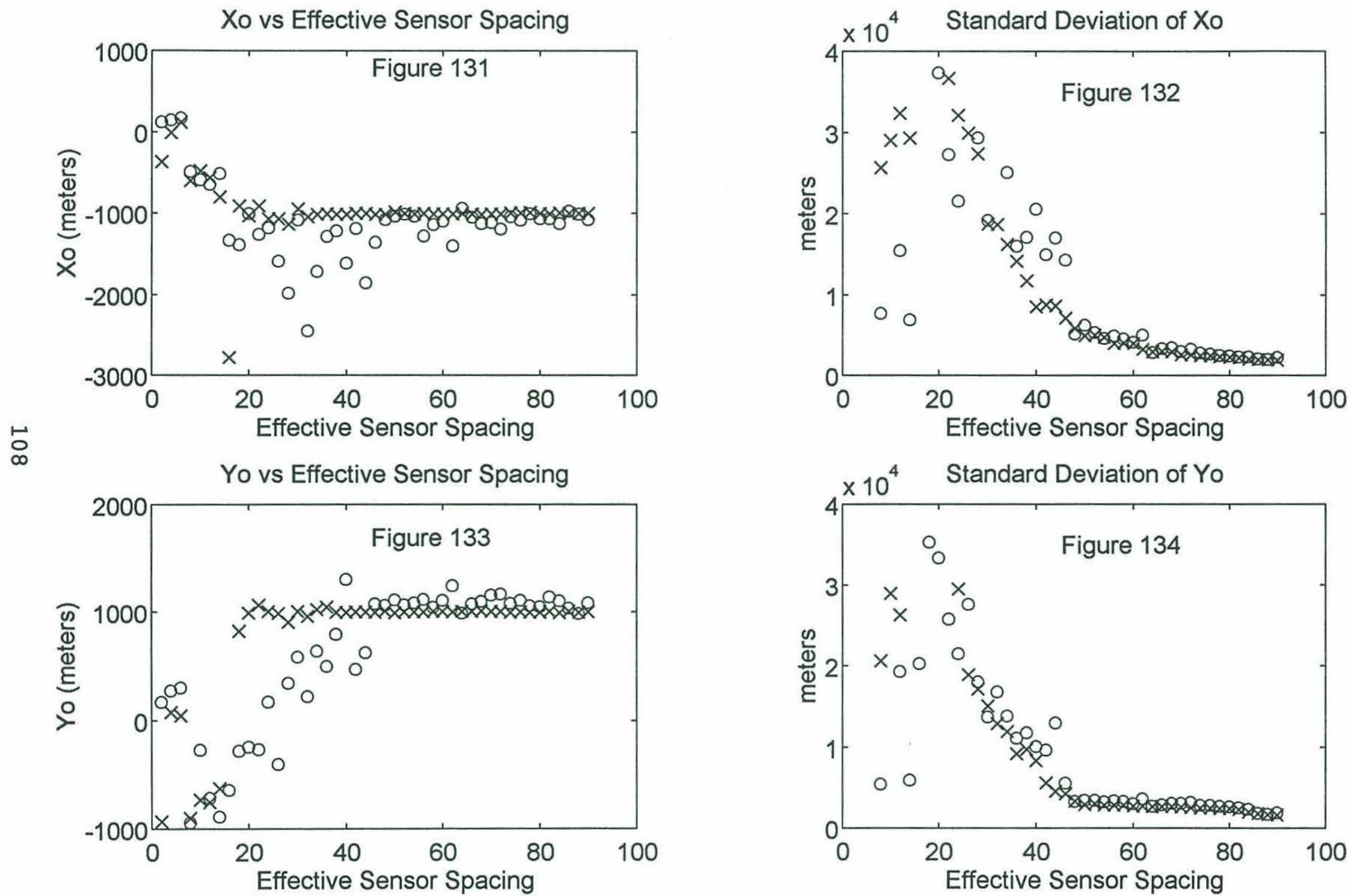

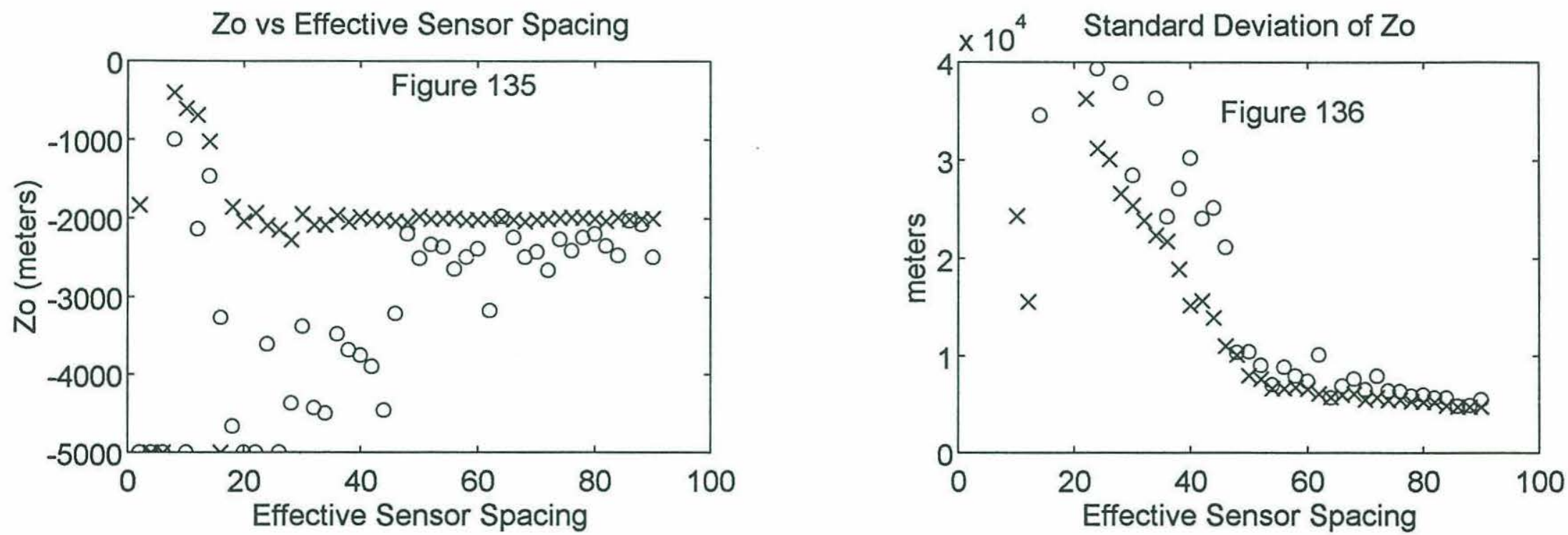

동
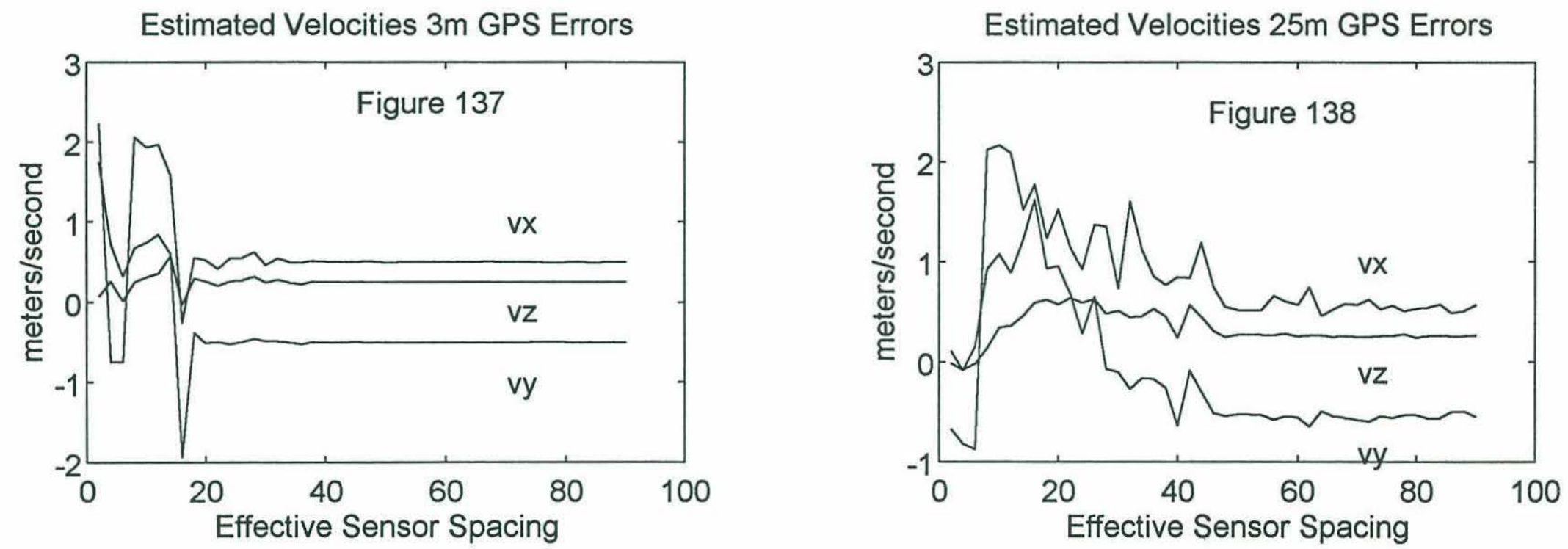

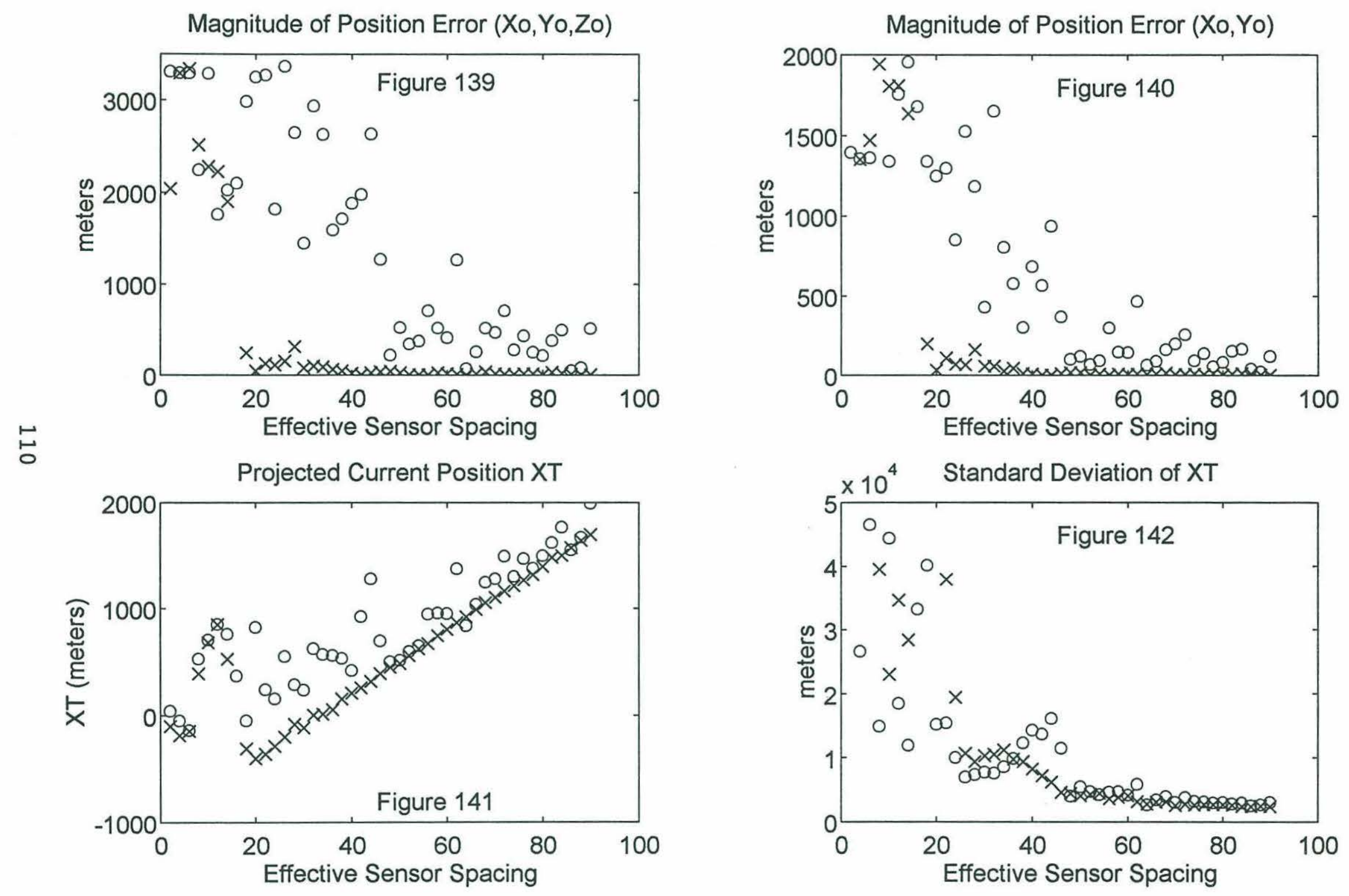

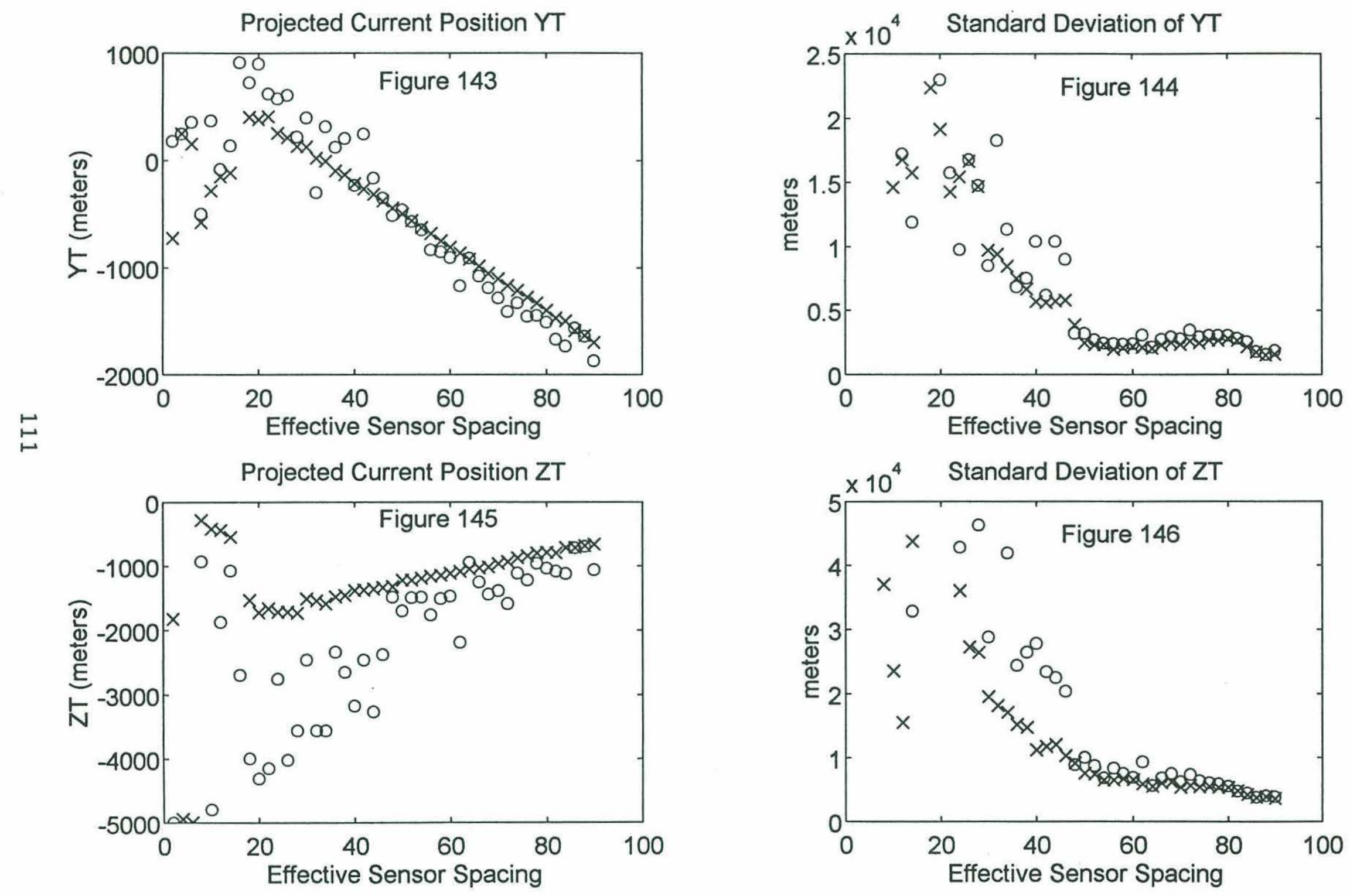


\subsubsection{Moving Acoustic Beacon Summary}

From scenarios 4 through 6 we showed that as the number of experiment vectors used in the maximum likelihood estimation problem increases, the accuracy of the estimated position and velocities of the acoustic beacon improves, while the standard deviation of the estimated position decreases. Also, as the effective sensor spacing increases, the accuracy of the estimated position and velocities of the acoustic beacon improves, and the standard deviation of the estimated position decreases. For all of the moving acoustic beacon scenarios, we see that the depth of the acoustic beacon is the least accurately estimated parameter. This is because we are still attempting to locate the acoustic beacon in three dimensions, while the receiving ship only moves in two dimensions. We also note that the standard deviations of the estimated beacon position are significantly larger for the moving beacon scenarios then for the stationary beacon scenarios. These larger standard deviations are due to the nature of the structural model and reflect the difficulties in locating a moving acoustic beacon using only a single omnidirectional hydrophone as a receiving source.

Similar to the stationary acoustic beacon scenarios, to obtain the best possible estimate of the acoustic beacon's location we need to use a large number of experiment vectors with a large effective sensor spacing. Again we run into problems with the time it takes to collect the required measurement vectors. For example, with an effective sensor spacing of 60 pulse repetition intervals, a pulse repetition interval of 2 seconds, and using 50 experiment vectors it takes 100 minutes to collect the required measurement vectors. As with the stationary acoustic beacon scenarios, if time is an 
issue, we must reduce the number of experiment vectors used, and reduce the effective sensor spacing. As a minimum, we must use at least 30 experiment vectors and a minimum effective sensor spacing of 45 pulse repetition intervals. With these values, for the search path of figure 4-1 and for a pulse repetition interval of 2 seconds, we should be able to obtain a decent estimate of the acoustic beacon's position and velocities.

\subsection{Summary}

From the results of the Monte Carlo simulations we see that in general, as the number of experiment vectors used to find the estimated parameter vector $\theta$ increases, the errors of the estimated parameters and the variance of the estimated parameter vector deceases. This implied that to obtain the best estimate of the acoustic beacon's location, we should use a large number of experiment vectors, with a large effective sensor spacing. We run into problems with this is approach if the time it takes to obtain an estimated position is a factor. We showed that for the search path given in section 4.2 and for an acoustic beacon with a pulse repetition interval of 2 seconds, we need to use a minimum of 20 experiment vectors and an effective sensor spacing of 35 pulse repetition intervals to obtain a decent estimate when using the stationary acoustic beacon algorithm. We also showed that with the same search path and pulse repetition interval, we need to use a minimum of 30 experiment vectors and an effective sensor spacing of 45 pulse repetition intervals. In all scenarios, the use of accurate navigational equipment to measure the position of the receiving ship is critical to obtain an accurate estimate of the acoustic beacon's location. This is shown by the 
better estimates obtained from using Differential/P code GPS errors of 3 meters rms. 


\section{Chapter V \\ Receiver Design}

\subsection{Introduction}

A receiver must be designed to implement the maximum likelihood estimation algorithms described in chapters II and III. This receiver should fulfill the goals of being an inexpensive, lightweight, easily transported receiver that can perform all of the functions of an older chart recorder. To determine the specifications for the receiver hardware, we look at the signal that is received, the ambient noise that is received, the thermal noise generated by the receiver's preamplifier, and the required signal output. We also examine the characteristics of the hydrophone used as a receiving sensor, and develop a practical hydrophone model so the preamplifier's input characteristics can be determined.

In designing a receiver to implement the algorithms described in chapters II and III, we make the following general assumptions. We assume that the beacon signal has a carrier frequency of $10,000 \mathrm{Hertz}$, a pulse width of 10 milliseconds, and a pulse repetition interval of 2 seconds. This is similar to several acoustic beacons currently in use. To cut costs, a Motorola $68 \mathrm{HC} 11$ microcontroller will be used. The $68 \mathrm{HC} 11$ is an 8-bit, low power microcontroller with several useful peripheral functions built in. These peripheral functions include an eight channel 8-bit analog to digital (A/D) converter, an asynchronous serial communications interface, five general input/output ports, and a 16-bit free running timer system with five output compare registers. This lets us use a single chip to synchronize the time of receipt $t_{\mu}$ to the 
pulse repetition interval, digitally sample the received signal, control the receiver's gain, and communicate with the interfacing computer. Another factor in deciding to use the $68 \mathrm{HC} 11$ microcontroller is that the $68 \mathrm{HC} 11$ and it's programming support equipment are commonly available. For the receiving sensor, we use the Benthos AQ-4 hydrophone attached to 100 meters of RG-58/u coaxial cable. The Benthos AQ-4 hydrophone has an open circuit response of $-201 \mathrm{~dB}$ re 1 Volt per $1 \mu \mathrm{Pa}$, which is representative of the type of omnidirectional hydrophones currently available.

\subsection{Design Criterion}

The signal transmitted by the acoustic beacon can be characterized as a sequence of gated continuous wave pulses at a carrier frequency $f_{c}$. We depict the beacon signal as:

$$
s_{n}(t)=\sum_{i=0}^{\infty} w\left(t-i T_{p}-T_{o}\right) \cdot \cos \left[2 \pi f_{c}\left(t-i T_{p}-T_{o}\right)+\phi\right]
$$

where $w(t)$ is a windowing function that defines the pulse width, $T_{p}$ is the period which defines the pulse repetition interval, and $\phi$ is a phase term. As before, the coefficient $T_{0}$ represents the unknown absolute time reference.

We can represent the signal that is received by the observer as:

$$
R_{n}(t)=\sum_{i=0}^{\infty} h_{i} \cdot w\left(t-i T_{p}-t_{s \mu}\right) \cdot \cos \left[2 \pi f_{c}\left(t-i T_{p}-t_{s \mu}\right)+\phi+\zeta_{i}\right]+n(t)
$$

where $h_{\mathrm{i}}$ represents the attenuation the signal experiences in traveling from the beacon to the observer, and $\zeta_{\mathrm{i}}$ represents a random phase distortion due the propagation of 
the signal through the ocean. As discussed in chapter II, the term $t_{s \mu}$ represents the synchronized time of receipt and is given by:

$$
t_{s \mu}=t_{\mu}-i \cdot T_{p}=T_{R}+T_{o}
$$

where $T_{R}$ is the acoustic travel time of the beacon signal. The term $n(t)$ represents the combination of the ambient noise that is received and the thermal noise that is generated by the receiver's preamplifier.

Since we use range differences in the maximum likelihood estimation algorithms of chapters II and III, the parameter that we need to find from the received signal is the synchronized time of receipt $t_{s \mu}$. In order to find $t_{s \mu}$, we digitally process the received signal. To determine the proper sampling rate, we must look at the frequency content of the received signal and the desired accuracy of the synchronized time of receipt $t_{s \mu}$. If we apply the Nyquist sampling rate theorem directly to the received signal, the sampling rate would have to be greater then twice the carrier frequency $f_{\mathrm{c}}$. For carrier frequencies around $10,000 \mathrm{Hertz}$, the required sampling rate is too fast for the $68 \mathrm{HC} 11$ to handle both the data collection and the signal processing. However, from looking at equation (5-2), we notice that the received windowing function $w(t)$ contains the parameter $t_{s \mu}$. Therefore, if we remove the carrier frequency and just look at the envelope of the received signal given by $w(t)$, we can still determine the synchronized time of receipt by observing when the signal's envelope is received. To remove the carrier frequency $f_{c}$, we use a precision rectifying circuit with a low pass filter to form an envelope detector. This allows us sample at the reduced rate of twice the bandwidth of the signal's envelope given by $w(t)$. 
To reduce the receiver's overall noise level, the bandwidth of the receiver should match the bandwidth of the envelope. If the bandwidth of the receiver increases above the bandwidth of the received signal's envelope, out of band noise from the oceanic environment and other interference sources enters the receiver, decreasing the signal to noise ratio. On the other hand, if the receiver's bandwidth is less then the bandwidth of the received signal's envelope, we do not allow the full bandwidth of the envelope to pass through the receiver, which again lowers the signal to noise ratio. The windowing function $\mathrm{w}(\mathrm{t})$ can be approximated by a simple rectangular window with a duration equal to the pulse width of the signal. The bandwidth of the windowing function and the receiver is then given by:

$$
\text { Bandwidth }=\frac{1}{\text { PulseWidth }}
$$

For a pulse width of 10 milliseconds, the windowing function's bandwidth is 100 Hertz. With a bandwidth of $100 \mathrm{Hertz}$, the sampling frequency must then be at least $200 \mathrm{Hertz}$, which equates to a sampling interval of no more then 5 milliseconds.

We must be careful not to sample at too slow a rate, otherwise the errors associated with the quantization of the time base will cause large errors in the localization algorithms. To determine an acceptable quantization step size, or equivalently, how much error can we tolerate in the time of receipt data, we need to compare the quantization errors to the errors in measuring the observer's location. The source of error in measuring the observers position is due to errors in the GPS position data. For a C/A-code GPS receiver, the published rms errors are on the order of 25 
meters. Using a simple homogeneous, direct path propagation model with the speed of sound in water equal to 1500 meters per second, a signal takes 16.667 milliseconds to travel 25 meters. We call this the equivalent time error of the position error. We would like the errors associated with the quantization of the time base to be less than the equivalent time error of the position errors. Assuming no other errors in the time of receipt, the maximum error in measuring the time of arrival $t_{s \mu}$ is half the sampling interval. For a C/A code GPS receiver the required sampling interval of 5 milliseconds is much less then the equivalent time error of the position error, therefore the quantization errors are relatively small compared to the errors in measuring the observer's position. However, because more accurate navigational equipment will most likely be available in the near future, and because the $68 \mathrm{HC} 11$ has the required speed, we will sample the received signal's envelope every 1 millisecond. With a sampling interval of 1 millisecond, as long as the rms errors of the navigational system are greater than 1.5 meters, the quantization errors will be smaller then the equivalent time errors of the position errors.

To determine the synchronized time of receipt $t_{s \mu}$ from the sampled envelope, we pass the sampled envelope through a matched filter based upon w(t), and look for the time that corresponds to the maximum value of the output of the matched filter. The received time is then divided modulo the pulse repetition interval to obtain the synchronized time of receipt. The use of a matched filter has two benefits. First, the output of a matched filter for a rectangular input signal with the same pulse width of $\mathrm{w}(\mathrm{t})$ will have a unique maximum peak which we can use to calculate the time of 
receipt. Secondly, a matched filter produces an output with the maximum signal to noise ratio. The digital form of a matched filter for a rectangular signal has the form of the moving average:

$$
y[n]=\frac{1}{N+1} \sum_{i=0}^{N} x[n-i]
$$

For a pulse width of 10 milliseconds and a sampling rate of 1 millisecond, $N$ is equal to 10 . Figures 5-1 and 5-2 show the output of the moving average matched filter for an input that represents the sampled envelope of the received signal with additive noise. From figure 5-2 we see that it is relatively easy to find the maximum value of the output of the matched filter. We do not have to worry about the phase delay of the matched filter because we compare the time of arrival of two signals relative to each other. Since the phase delay is the same for all received signals, it does not effect the comparison of the two arrival times.

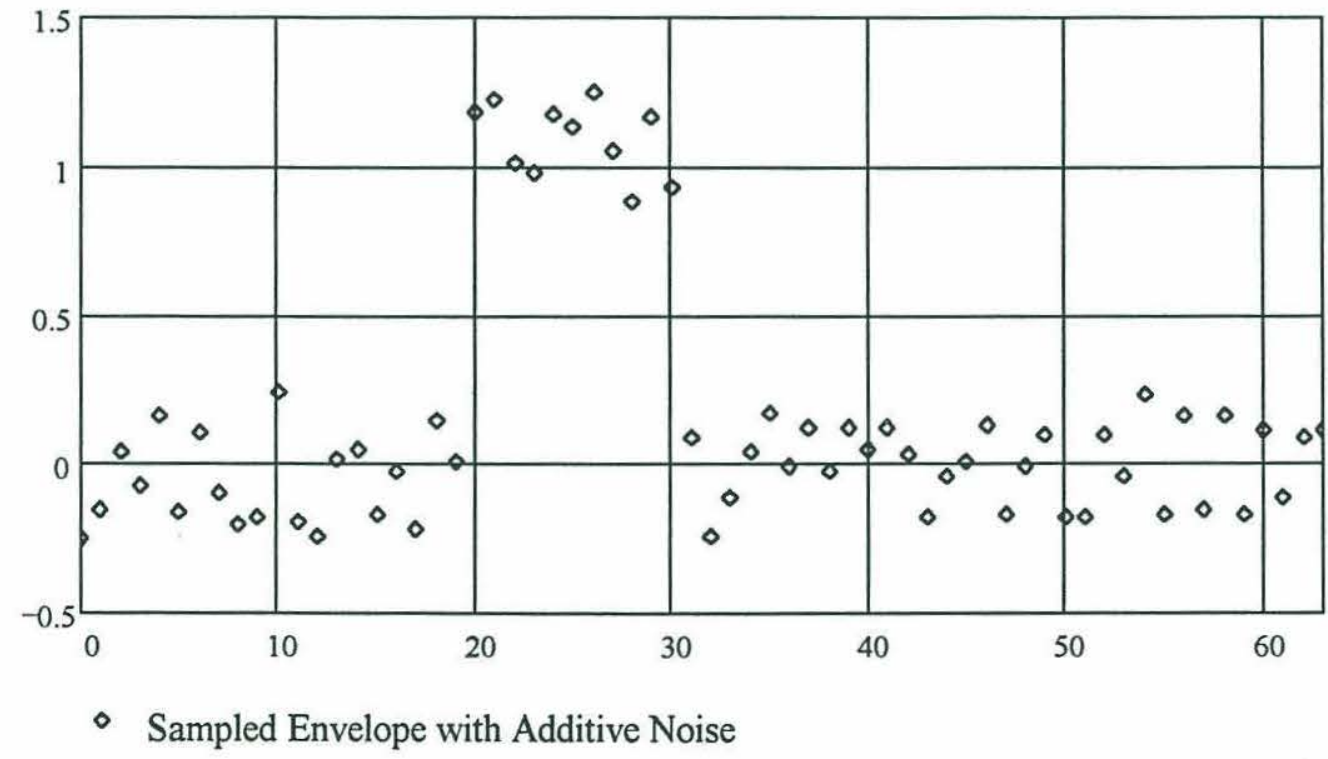

Figure 5-1 


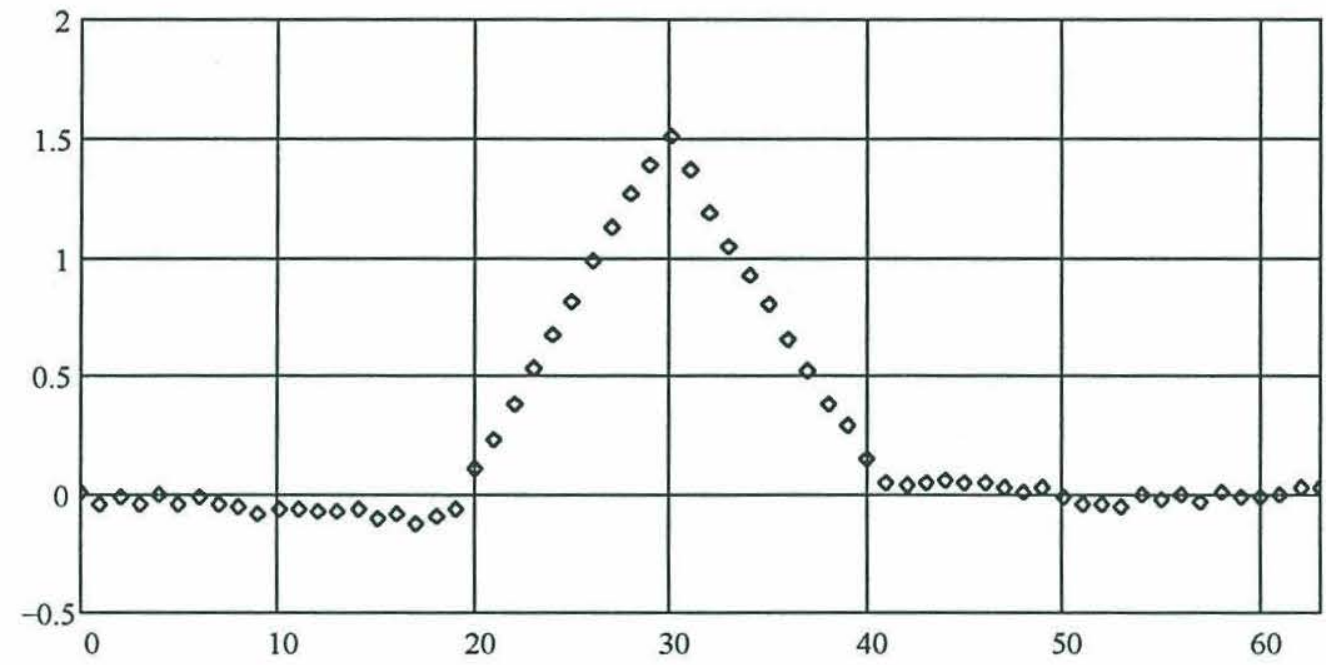

- Output of Matched Filter

Figure 5-2

The receiver should be able to detect the target's beacon signal in the presence of the additive noise term $\mathrm{n}(\mathrm{t})$. The two largest contributors of noise are ambient oceanic noise and thermal noise generated by the receiver's preamplifier. Ambient oceanic noise, due to factors such as wind force, ships, industrial activity, precipitation, biologics, and others, is received by the hydrophone which generates an equivalent noise voltage. Using the hydrophone's open circuit response characteristics, the receiver's bandwidth, and empirical data of noise spectrum levels summarized by Wenz [Wenz 1962], the equivalent noise voltage of the oceanic noise can be calculated using:

$$
e_{n}=10^{\left(\frac{H_{o c}+e_{o}}{20}+\log (B W)\right)}
$$

where $\mathrm{H}_{\mathrm{oc}}$ is the hydrophones open circuit response in $\mathrm{dB}$ re 1 Volt per $1 \mu \mathrm{Pa}, \mathrm{e}_{\mathrm{o}}$ is the 
ambient oceanic noise in $\mathrm{dB}$ re $1 \mu \mathrm{Pa}$, and $\mathrm{BW}$ is the receiver's bandwidth in Hertz.

Table 5-1 shows the equivalent noise voltage for various Sea states (Beaufort Scale) at 10,000 Hertz using a hydrophone with an open circuit receiver response of $-201 \mathrm{~dB}$ re 1 Volt per $1 \mu \mathrm{Pa}$, and a receiver bandwidth of 100 Hertz. Ideally the noise in a receiver should be dominated by external noise sources. This means that the receiver's thermal noise should be less then the ambient oceanic noise. Since sea states 1 and 2 are not common, the receiver's thermal noise is designed to be less then the ambient oceanic noise associated with sea state 3 . This requirement limits the test receiver's thermal noise reflected at the input of the preamplifier to be less then $89 \mathrm{nV} / \sqrt{\mathrm{Hz}}$.

\begin{tabular}{||c|c|c|c||}
\hline $\begin{array}{c}\text { Sea State } \\
\text { (Beaufort Scale) }\end{array}$ & $\begin{array}{c}\text { Ambient Oceanic } \\
\text { Noise } \\
(\mathbf{d B} \text { re } \mathbf{1} \boldsymbol{\mu P a})\end{array}$ & $\begin{array}{c}\text { Equivalent } \\
\text { Noise Voltage } \\
(\mathbf{n V} / \sqrt{\mathbf{H z})}\end{array}$ & $\begin{array}{c}\text { Total Equivalent } \\
\text { Noise Voltage } \mathbf{e}_{\mathbf{n}} \\
(\boldsymbol{\mu} \mathbf{V})\end{array}$ \\
\hline \hline 1 & 48 & 22 & 0.22 \\
\hline 2 & 56 & 56 & 0.56 \\
\hline 3 & 60 & 89 & 0.89 \\
\hline 5 & 68 & 224 & 2.24 \\
\hline 8 & 73 & 398 & 3.98 \\
\hline limit & 80 & 891 & 8.91 \\
\hline
\end{tabular}

Table 5-1

Another desirable characteristic of the receiver is that it should have as large a dynamic resolution as possible. A large dynamic resolution allows the receiver to detect a broad level of signal amplitudes for a set gain. Since the received signal will be digitally processed, we need to look at the dynamic resolution of the A/D converter. 
The dynamic resolution of an A/D converter is given by:

$$
\text { Dynamic Resolution }=20 \log _{10}\left(2^{\text {bits }}\right)
$$

For and 8 bit A/D converter, the dynamic resolution is only

$$
20 \log _{10}\left(2^{8}\right)=48 d B
$$

This is not quite large enough. We would like a dynamic resolution that is comparable to the dynamic resolution of the older paper recorders. This means we need a dynamic resolution of at least $60 \mathrm{~dB}$. To increase the dynamic resolution, either a higher bit A/D converter could be used, or a logarithmic compressor can be used prior to digital sampling. Since we are restricted to the 8 bit A/D converter of the $68 \mathrm{HC} 11$, a logarithmic compressor will be used to increase the receiver's dynamic resolution.

Using a logarithmic compressor, the dynamic resolution is given by:

$$
\text { Dynamic Resolution }=20 \cdot \log _{10}\left(\frac{V_{\text {sat }}}{V_{\min }}\right)
$$

where $\mathrm{V}_{\text {sat }}$ is the saturation point of the logarithmic compressor and $\mathrm{V}_{\min }$ is the minimum detectable signal at the input of the logarithmic compressor. Typically, logarithmic compressors require an input signal that is greater then $2 \mathrm{mV}$. Below 2 $\mathrm{mV}$, the input current to the logarithmic compressor becomes comparable to the bias current of the matched transistors used to obtain the logarithmic dependence, and 
subsequently the logarithmic dependence is no longer valid [Millman 1979]. For the receiver designed in section $5.4, \mathrm{~V}_{\text {sat }}$ is approximately $8 \mathrm{~V}$ which gives a dynamic resolution of:

$$
\text { Dynamic Resolution }=20 \cdot \log _{10}\left(\frac{8}{2 \cdot 10^{-3}}\right)=72 \mathrm{~dB}
$$

To determine the required gain of the receiver, we need to look at the signal level that we are attempting to receive. We wish to be able to detect low level signals that are right at the noise floor of the receiver. This requires the receiver to have enough gain to detect a signal at the equivalent oceanic noise level of $89 \mathrm{nV} / \sqrt{\mathrm{Hz}}$. Since a logarithmic compressor is used just prior to A/D conversion, the required gain is determined by the minimum detectable level in the logarithmic compressor and the oceanic noise level. Using the ambient oceanic noise and the input requirements of the logarithmic compressor from above, the maximum required gain of the receiver is given by:

$$
A_{v_{\max }}=\frac{2 \cdot 10^{-3}}{89 \cdot 10^{-9}}=87 \mathrm{~dB}
$$

However, it is possible that the received signal could be quite a bit larger then the minimum signal level for a strong beacon in close proximity to the receiving ship. For example, a $30 \mathrm{Watt}$ beacon 100 meters from the receiving hydrophone will produce an input signal of about $10 \mathrm{mV}$. This signal would completely saturate the receiver if the gain is fixed at $A_{V_{\max }}$. To prevent the receiver from saturating, some form of feedback gain control is needed. Using one of the output ports of the $68 \mathrm{HC} 11$ 
as a control line, simple resistive attenuators can be switched on or off to reduce or increase the gain of the receiver based upon the maximum signal level at the A/D converter. To determine how much attenuation is needed, we look at the saturation point of the preamplifier and the maximum likely input signal. For design purposes, to keep a $10 \mathrm{mV}$ signal from saturating the receiver, the receiver's minimum gain is given by:

$$
A_{v_{\min }}=\frac{V_{\text {sat }}}{10 \cdot 10^{-3}}=\frac{8}{10 \cdot 10^{-3}}=58 d B
$$

Here again $\mathrm{V}_{\text {sat }}$ is the logarithmic compressor's saturation level. Since the input signal level will be somewhere between the noise floor and the maximum likely input signal most of the time, we can use multiple resistive attenuator networks to set the receiver's gain to any level in between $A_{V_{\max }}$ and $A_{V_{\min }}$. For this design we will use four resistive attenuator networks to provide attenuation levels of $-3 \mathrm{~dB},-9 \mathrm{~dB}$, $-12 \mathrm{~dB},-18 \mathrm{~dB},-24 \mathrm{~dB}$, and $-30 \mathrm{~dB}$.

\subsection{Hydrophone Model}

To determine the input characteristics of the preamplifier, the hydrophone and cable must be modeled. Figure 5-3 shows an equivalent circuit for a stiffnesscontrolled piezoelectric hydrophone. Here $R_{h}$ is the hydrophone's resistance, $C_{h}$ is the hydrophone's capacitance, $e_{n}$ represents the equivalent ambient noise of the ocean, and $e_{h n}$ represents the thermal noise of the hydrophone. For frequencies considerably below $100 \mathrm{kHz}$, the ambient noise level of the ocean is much greater then the 
hydrophone's thermal noise, while the impedance of the hydrophone is dominated by the capacitance $\mathrm{C}_{\mathrm{h}}$. This allows us to simplify the hydrophone model by eliminating $\mathrm{R}_{\mathrm{h}}$ and $\mathrm{e}_{\mathrm{hn}}$ [Wilson 1985].

\section{Figure 5-3}

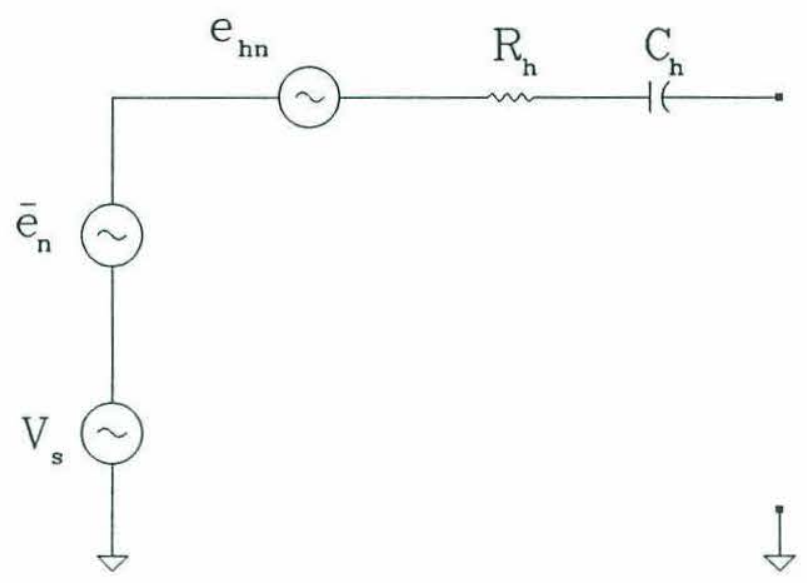

The cable assembly can be modeled as a two conductor transmission line.

Using standard RG-58/u coaxial cable with a solid copper inner conductor and a polyethylene dielectric, we can model the cable as a series resistance $R_{c}$ and a shunt capacitance $\mathrm{C}_{\mathrm{c}}$ [Cheng 1983]. Standard RG-58/u coaxial cable has a series resistance of $33.31 \Omega$ per 1,000 meters, and shunt capacitance of $70 \mathrm{pF}$ per meter. Combing the simplified hydrophone model and the cable model, we have the circuit of figure 5- 4.

Figure 5-4

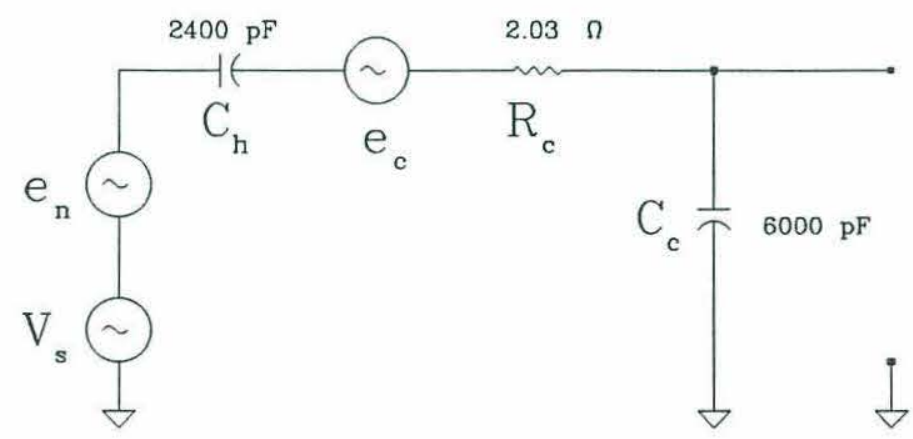


Figure 5-4 can be simplified to give the equivalent Thevinin circuit model:

Figure 5-5

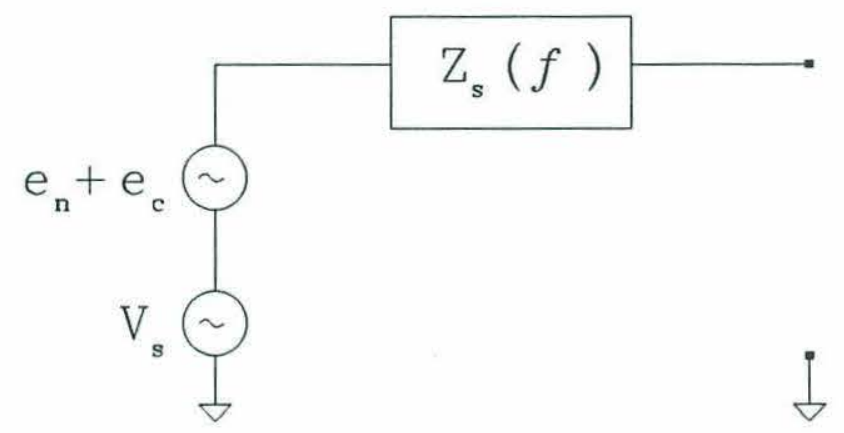

The source impedance $Z_{\mathrm{s}}(f)$ is given by:

$$
Z_{s}(f)=\frac{2 j \pi f R_{c} C_{h}+1}{\left[2 j \pi f R_{c} C_{h} C_{c}+\left(C_{h}+C_{c}\right)\right] 2 j \pi f}
$$

Figure 5-6 shows a plot of the source impedance verses frequency for the Benthos AQ-4 hydrophone with $\mathrm{C}_{\mathrm{h}}$ specified as $2,400 \mathrm{pF} \pm 25 \%$. To prevent serious loading effects, the preamplifier's input impedance must be considerably larger then the source impedance given by equation (5-13). For design purposes, the magnitude of the source impedance at $10,000 \mathrm{Hertz}$ is $1,693 \Omega$, therefore we choose the preamplifier's input impedance to be at a minimum of 20 time the source impedance, or $33.8 \mathrm{k} \Omega$. This will keep the loss due to loading below $0.5 \mathrm{~dB}$. 


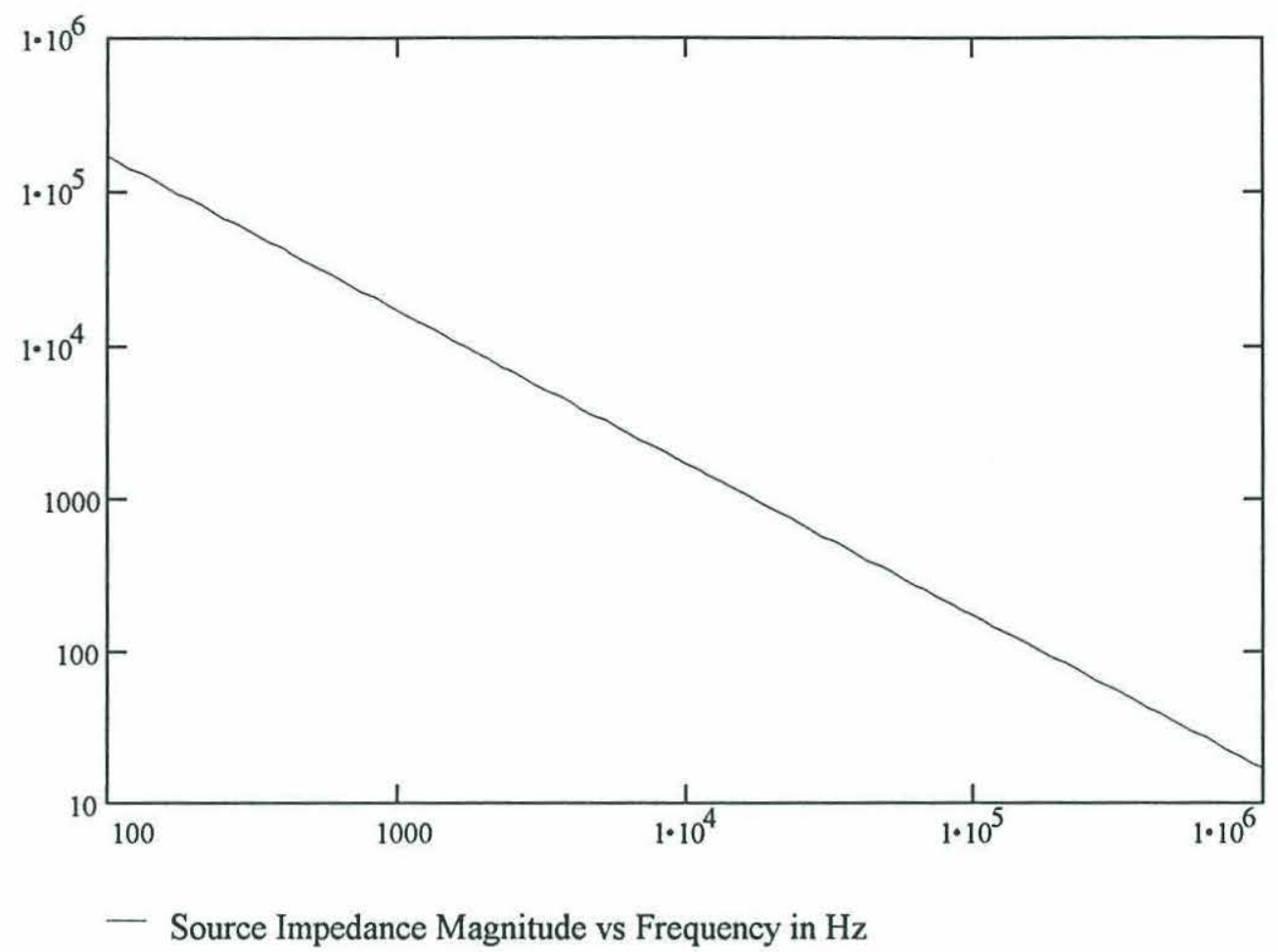

Figure 5-6

\subsection{Test Receiver Hardware Design}

Based on these design criteria, a receiver was built for testing. This section discuses the circuit design considerations and implementation of this test receiver. Figure 7 shows the general signal flow of the receiver starting with the preamplifier and moving to the $68 \mathrm{HC} 11$ microcontroller. An important theme of the receiver design is to avoid exotic components to minimize the cost of the receiver.

\subsubsection{Preamplifier}

Refer to the preamplifier schematic diagrams in Appendix 1 for the following description. The first stage of the preamplifier is a tuned $\mathrm{N}$ channel junction field effect transistor (JFET) common-source amplifier. To help keep low frequency ambient oceanic noise from entering the preamplifier, $\mathrm{C}_{01}$ and $\mathrm{R}_{01}$ form a high pass 


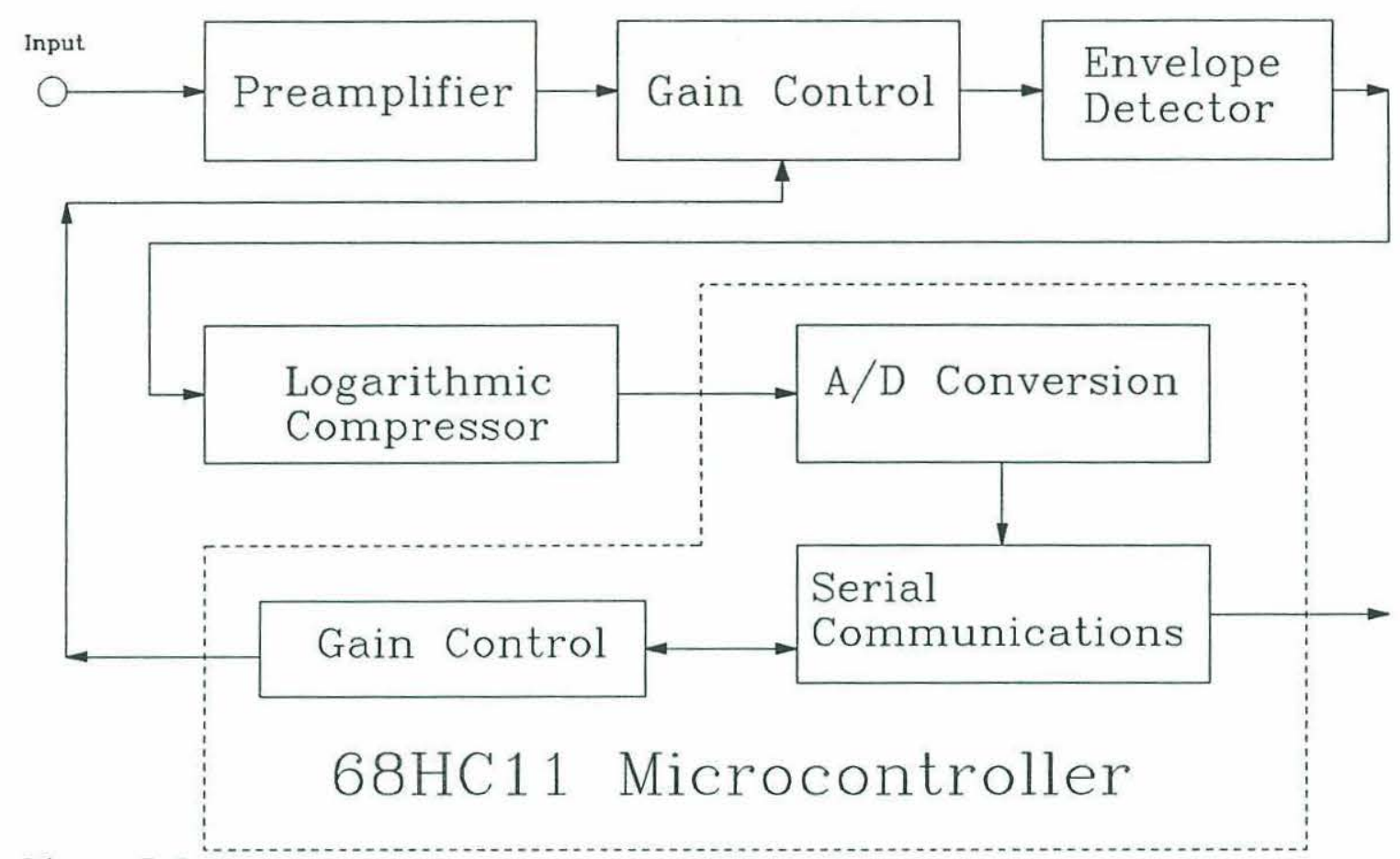

Figure 5-7

filter with a cutoff frequency given by:

$$
f_{c}=\frac{1}{2 \pi R_{01} C_{01}}
$$

To eliminate as much low frequency oceanic noise as possible but still allow for small changes in the acoustic beacon's carrier frequency, we chose the high pass filter cut off frequency as 7000 Hertz. The preamplifier's input impedance is determined by $R_{01}$ since the input impedance of the JFET is so large $\left(>10^{14} \Omega\right)$. To prevent loading between the hydrophone source and the preamplifier we must ensure $R_{01}$ is larger then $33.8 \mathrm{k} \Omega$. To ensure that the thermal noise of the preamplifier is less then equivalent oceanic noise of sea state $3, R_{01}$ must be chosen such that the thermal noise of the parallel combination of $\mathrm{R}_{01}$ and source impedance $\mathrm{Z}_{\mathrm{s}}(f)$ is considerably less then 
$89 \mathrm{nV} / \sqrt{\mathrm{Hz}}$. The thermal noise of a resistor is given by:

$$
\bar{e}_{n}=\sqrt{4 k t R B}
$$

where $\mathrm{k}$ is Boltzmann's constant, $\mathrm{t}$ is the absolute temperature in Kelvin, $\mathrm{B}$ is the bandwidth in Hertz, and $\mathrm{R}$ is the resistance in Ohms [Horowitz and Hill 1989]. For the Benthos AQ-4 hydrophone at a frequency of 10,000 Hertz $Z_{s}(f)$ is approximately $1700 \Omega$. As a result, the parallel combination of $\mathrm{R}_{01}$ and $Z_{s}(f)$ will be close to the value of $Z_{\mathrm{s}}(f)$ for $\mathrm{R}_{01}$ greater then $32 \mathrm{k} \Omega$, and the associated thermal noise will be well below $89 \mathrm{nV} / \sqrt{\mathrm{Hz}}$. However, it would be nice to ensure that the thermal noise of the preamplifier is much less then equivalent oceanic noise of sea state 3 for any source impedance. To ensure this, we choose $R_{01}$ such that its thermal noise is approximately $45 \mathrm{nV} / \sqrt{\mathrm{Hz}}$. To meet the noise constraint, the loading constraint, and the cut off frequency constrain of equation (14), we choose $R_{01}$ as $120 \mathrm{k} \Omega$ and $C_{01}$ as $180 \mathrm{pF}$.

The gain of the first stage JFET common-source amplifier is given by:

$$
A_{v_{1}}(f)=\frac{-Y_{f s} \cdot Z_{D}(f) \cdot r_{d}}{Z_{D}(f)+r_{d}}
$$

where $\mathrm{Y}_{\mathrm{fs}}$ is the JFET's forward transconductance, $\mathrm{r}_{\mathrm{d}}$ is the JFET's intrinsic drain resistance, and $Z_{D}(f)$ is given by:

$$
Z_{d}(f)=\frac{\frac{2 j \pi f}{C_{02}}}{(j \pi f)^{2}+\frac{2 j \pi f}{R_{02} C_{02}}+\frac{1}{L_{01} C_{02}}}
$$


$Z_{D}(f)$ represents the complex impedance of the combination of $\mathrm{L}_{01}, \mathrm{R}_{02}$, and $\mathrm{C}_{02}$. The use of the reactive load $Z_{\mathrm{D}}(f)$ allows us to obtain the narrow bandwidth required by equation (5-4). The gain of the first stage will be greatest when the frequency of the input signal is at the resonant frequency of $Z_{\mathrm{D}}(f)$. The resonant frequency of $Z_{\mathrm{D}}(f)$ is given by:

$$
f_{r}=\frac{1}{2 \pi \sqrt{L_{01} C_{02}}}
$$

We want the preamplifier to have it's maximum gain at the acoustic beacon's carrier frequency, therefore we choose $\mathrm{L}_{01}$ and $\mathrm{C}_{02}$ such that the resonant frequency equals the acoustic beacon's carrier frequency. In the test receiver, $\mathrm{Q}_{01}$ is an E230 general purpose $\mathrm{N}$ channel JFET. The E230 has a nominal forward transconductance of $1.5 \cdot 10^{-3}$ Siemens and a nominal intrinsic drain resistance of $12.5 \mathrm{k} \Omega$. Using equations (5-16) and (5-17), and the values from Appendix 1, at a beacon frequency of 10,000 $\mathrm{Hz}, \mathrm{A}_{\mathrm{V} 1}$ is approximately $24 \mathrm{~dB}$.

In general, the output impedance of an R-L-C tuned amplifier is quite high, therefore, a JFET source follower is used as a buffer immediately following the first stage. Resistor $\mathrm{R}_{04}$ and capacitor $\mathrm{C}_{04}$ form a high pass filter similar to $\mathrm{R}_{01}$ and $\mathrm{C}_{01}$, and serve to capacitively couple the JFET source follower to the tuned JFET amplifier. The cut off frequency of the high pass filter formed by $\mathrm{R}_{04}$ and $\mathrm{C}_{04}$ is given by equation (5-14) with $\mathrm{R}_{04}$ substituted for $\mathrm{R}_{01}$ and $\mathrm{C}_{04}$ substituted for $\mathrm{C}_{01}$. To prevent loading between the JFET amplifier and the JFET buffer, $\mathrm{R}_{04}$ must be significantly larger then the output impedance of the JFET amplifier. However, we must be careful 
in choosing $\mathrm{R}_{04}$ so that its thermal noise, when reflected back to the input of the first stage, is less then $89 \mathrm{nV} / \sqrt{ } \mathrm{Hz}$. Using equation (15), this limits $R_{04}$ to $560 \mathrm{k} \Omega$.

To enhance the performance of the JFET source follower, $Q_{03}$ and $R_{05}$ are added as a bootstrapped load. Because the base-emitter voltage of $Q_{03}$ is approximately constant, the combination of $\mathrm{R}_{05}$ and $\mathrm{Q}_{03}$ cause a constant source current to flow through JFET $\mathrm{Q}_{02}$. This makes $\mathrm{Q}_{02}{ }^{\prime} \mathrm{S} \mathrm{V}_{\mathrm{GS}}$ approximately constant, which in return reduces nonlinearities [Horowitz and Hill 1989]. This is beneficial because the signal level at this point in the preamplifier is still quite small.

The first stage of the preamplifier is powered by a separate 5 Volt 3 -terminal 7805 voltage regulator. We do this to isolate the first stage from subsequent stages, which prevents the feedback of any noise generated by subsequent stages along the main power supply line. This provides $\mathrm{Q}_{01}$ with a clean, stable supply voltage which is critical when working with very low level input signals.

The second stage of the preamplifier is another tuned amplifier, this time using a PNP bipolar transistor. Capacitor $\mathrm{C}_{05}$ combined with the parallel combination of the bias resistors $R_{07}$ and $R_{08}$ form another high pass filter while also serving to capacitively couple the JFET source follower from the first stage to the second stage. The cutoff frequency of the high pass filter formed by $C_{05}, R_{07}$ and $R_{08}$ is given by:

$$
f_{c}=\frac{1}{2 \pi \cdot C_{05} \frac{R_{07} \cdot R_{08}}{R_{07}+R_{08}}}
$$


For this stage, we set the high pass filter's cut off frequency at $3000 \mathrm{Hertz}$. The gain of the second stage is given by:

$$
A_{V_{2}}=\frac{-Z_{c}(f)}{R_{10}}
$$

where

$$
Z_{c}(f)=\frac{\frac{2 j \pi f}{C_{07}}}{(2 j \pi f)^{2}+\frac{2 j \pi f}{R_{11} C_{07}}+\frac{1}{L_{02} C_{07}}}
$$

As in the first stage we set the resonant frequency of $Z_{c}(f)$ given by:

$$
f_{r}=\frac{1}{2 \pi \sqrt{L_{02} C_{07}}}
$$

to the acoustic beacon's carrier frequency. Using equations (5-20) and (5-21) for a beacon frequency of 10,000 Hertz, $A_{\mathrm{V}_{2}}$ is approximately $31 \mathrm{db}$.

Following the PNP tuned amplifier, a simple JFET source follower is used as a buffer. Capacitor $\mathrm{C}_{08}$ and resistor $\mathrm{R}_{12}$ form yet another high pass filter while also capacitively coupling the output of the PNP amplifier to the input to the JFET follower. The cut off frequency of the high pass filter formed by $C_{08}$ and $R_{12}$ is given by equation (14) with $C_{08}$ substituted for $C_{01}$ and $R_{12}$ substituted for $R_{01}$, and is set to 3,000 Hertz.

Similar to the first stage, the second gain stage is powered by a separate 5 Volt, 3-terminal 7805 voltage regulator. Again we do this to prevent noise from being feedback along the power supply lines. 
At the end of the second stage of the preamplifier the maximum gain at the acoustic beacon's carrier frequency is approximately $55 \mathrm{~dB}$. We are under the minimum required gain of $58 \mathrm{~dB}$, therefore we should not have to worry about the first two stages of the preamplifier saturating the logarithmic compressor. However, we still need an additional $32 \mathrm{~dB}$ of gain to reach the required maximum gain of 87 dB. Prior to the next gain stage, we include an attenuation network to prevent subsequent stages from saturating.

Following the JFET source follower $\mathrm{Q}_{05}, \mathrm{R}_{14}$ in combination with shunt resistors $R_{15}, R_{16}, R_{17}$, and $R_{18}$ form a variable attenuation network which is controlled by the $68 \mathrm{HC} 11$ microcontroller. To reduce the gain of the preamplifier, any combination of $R_{15}$ through $R_{18}$ can be switched to ground through two CD4053 analog switches. Once grounded, the shunt resistors form a voltage divider with $R_{14}$, and subsequently attenuate the signal. Following the shunt resistors is a simple common emitter amplifier that provides the final gain necessary to obtain a maximum of $87 \mathrm{~dB}$. The gain of this stage, including the effects of the attenuator network is given by:

$$
A_{V_{3}}=\frac{-R_{21}}{R_{22}} \cdot \frac{2 \pi f R_{s} C_{09} \cdot \frac{R_{19} R_{20}}{R_{19}+R_{20}}}{\left[\left(R_{14}+\frac{R_{19} R_{20}}{R_{19}+R_{20}}\right) \cdot R_{s}+\frac{R_{19} R_{20}}{R_{19}+R_{20}} \cdot R_{14}\right] \cdot 2 \pi f C_{09}+R_{s}+R_{14}}
$$

where $R_{s}$ is the parallel combination of any shunt resistors $R_{15}$ through $R_{18}$ that are switched to ground. If none of the shunt resistors are switched to ground, $\mathrm{R}_{\mathrm{s}}$ is very large and $\mathrm{A}_{\mathrm{V} 3}$ approaches: 


$$
A_{V_{3}}=\frac{-R_{21}}{R_{22}}
$$

In this case the gain of the preamplifier is at its maximum of approximately $89 \mathrm{~dB}$. In actuality, the maximum gain will be slightly less then $89 \mathrm{~dB}$ due to small losses caused by loading between the different stages. If all of the shunt resistors are switched to ground, $R_{s}$ is at its minimum value and the gain of the preamplifier is approximately $56 \mathrm{~dB}$. Table $5-2$ summarizes which shunt resistors are switched to ground to obtain different levels of attenuation.

\begin{tabular}{||c|c||}
\hline Attenuation Level & Shunt Resistors Switched to Ground \\
\hline $0 \mathrm{~dB}$ & none \\
\hline$-3 \mathrm{~dB}$ & $\mathrm{R}_{15}$ \\
\hline$-9 \mathrm{~dB}$ & $\mathrm{R}_{16}$ \\
\hline$-12 \mathrm{~dB}$ & $\mathrm{R}_{15}$ and $\mathrm{R}_{16}$ \\
\hline$-18 \mathrm{~dB}$ & $\mathrm{R}_{17}$ \\
\hline$-24 \mathrm{~dB}$ & $\mathrm{R}_{18}$ \\
\hline$-30 \mathrm{~dB}$ & $\mathrm{R}_{15}, \mathrm{R}_{16}, \mathrm{R}_{17}$, and $\mathrm{R}_{18}$ \\
\hline
\end{tabular}

Table 5-2

\subsubsection{Envelope Detector Circuit}

Refer to the Envelope Detector schematic diagram in Appendix 1 for the following description. After the preamplifier, the received signal passes through a unity gain buffer. This isolates the preamplifier from the envelope detector, and also provides the low source impedance that the precision rectifier circuit needs to function properly. $\mathrm{U}_{07}, \mathrm{Q}_{07}, \mathrm{Q}_{08}$, and the low pass filter combination of $\mathrm{C}_{25}$ and $\mathrm{R}_{31}$ are the 
heart of the envelope detector. Diode $\mathrm{D}_{01}$ protects $\mathrm{U}_{07}$ during large input swings above the reference voltage $V_{\text {REF }}$. Resistors $R_{29}$ and $R_{30}$ add bias stability to transistors $Q_{07}$ and $\mathrm{Q}_{08}$.

As the input signal at the inverting terminal of $U_{07}$ goes below the reference voltage at the noninverting terminal of $\mathrm{U}_{07}, \mathrm{Q}_{07}$ is brought into the active region, thereby drawing $\mathrm{Q}_{08}$ into the active region which charges capacitor $\mathrm{C}_{25}$. As the difference between the reference voltage and the input signal becomes greater, $\mathrm{Q}_{07}$ and $\mathrm{Q}_{08}$ are brought further into the active region which in turn increases the time that capacitor $\mathrm{C}_{25}$ charges. When the signal at the inverting terminal of $\mathrm{U}_{07}$ is above the reference voltage at the noninverting terminal of $U_{07}$, transistor $Q_{07}$, and subsequently $\mathrm{Q}_{08}$ are cut off. The charge in capacitor $\mathrm{C}_{25}$ is then discharged through resistor $\mathrm{R}_{31}$. As long as the time constant formed by the combination of $\mathrm{C}_{25}$ and $\mathrm{R}_{31}$ is much greater then the period of the input signal's carrier frequency, the output taken at $\mathrm{C}_{25}$ will be the envelope of the input signal. To ensure operation with very low level inputs, $\mathrm{U}_{08}$ is chosen to have a large gain bandwidth product and a fast slew rate.

\subsubsection{Logarithmic Compressor}

Refer to the Logarithmic Compressor schematic diagram in Appendix 1 for the following description. Prior to logarithmic compression, we pass the received envelope through a noninverting amplifier with a gain of $9 \mathrm{~dB}$ to restore the signal level that was lost in the envelope detector. To obtain an output that is proportional the logarithm of the input, we rely on the logarithmic relation between transistor $\mathrm{Q}_{09}$ 's voltage and current. By using $\mathrm{Q}_{09}$ in $\mathrm{U}_{09}$ 's feedback loop, the output of the logarithmic 
compressor is given by:

$$
V_{o}=-\left(1+\frac{R_{41}}{R_{42}}\right) \cdot V_{T} \cdot \ln \left(\frac{V_{i} \cdot R_{40}}{R_{35} \cdot V+}\right)
$$

where $\mathrm{V}_{\mathrm{T}}$ is given by:

$$
V_{T}=\frac{\text { temperature }}{11600}
$$

Here the temperature is in Kelvin. For room temperature, $\mathrm{V}_{\mathrm{T}}$ is approximately 0.0259 . From equation (5-25) we note that for small input signals, the output $\mathrm{V}_{\mathrm{o}}$ is large, and for large input signals, the output $\mathrm{V}_{\mathrm{o}}$ is small. To set the zero crossing point of the logarithmic compressor, we choose $\mathrm{R}_{40}$ such that:

$$
R_{40}=\frac{R_{35} \cdot V+}{V_{\text {sat }}}
$$

With $\mathrm{R}_{35}$ equal to $10 \mathrm{k} \Omega, \mathrm{V}+$ equal to 10 Volts, and $\mathrm{V}_{\text {sat }}$ equal to 8 Volts, $\mathrm{R}_{40}$ is approximately $12.5 \mathrm{k} \Omega . \mathrm{V}_{\text {sat }}$ represents the largest possible input to the logarithmic compressor. To set the maximum output level of the logarithmic compressor, we chose $R_{42}$ and $R_{43}$ such that the minimum input signal of $2 \mathrm{mV}$ produces an output of 5 volts. This allows us to digitally sample the output of the logarithmic compressor directly, without worrying about damaging the $68 \mathrm{HC} 11$ 's A/D converter. The use of the matched transistor $\mathrm{Q}_{09}$ helps stabilize the temperature dependence of $\mathrm{V}_{\mathrm{T}}$, and is essential to eliminate the dependence upon the reverse saturation current $I_{\mathrm{o}}$ which doubles for every $10^{\circ} \mathrm{C}$ rise in temperature [Millman 1979].

By taking the saturation level of the receivers as approximately 8 volts, the 
dynamic resolution of the receiver is approximately:

$$
\text { Dynamic Resolution }=20 \cdot \log _{10}\left(\frac{8}{2 \cdot 10^{-3}}\right)=72 \mathrm{~dB}
$$

To obtain the full dynamic resolution at the input to the $A / D$ converter, the output of operational amplifier $\mathrm{U}_{10}$ must be capable of going between zero and five volts. We would like to restrict the output of $\mathrm{U}_{10}$ to be strictly less then 5.5 Volts to prevent damage to the $68 \mathrm{HC} 11$ 's $\mathrm{A} / \mathrm{D}$ converter in case of a failure in the logarithmic compressor. To accomplish this, we step down the main positive supply voltage to 5.6 volts using a zener diode reference. The maximum output of the CA3130 operational amplifier used for $U_{10}$ is about 0.5 volts below the positive supply voltage, therefore the output of $\mathrm{U}_{10}$ is restricted between 0 and 5.1 volts.

\subsubsection{HC11 Microcontroller}

Refer to the $68 \mathrm{HC} 11$ Microcontroller schematic diagram in Appendix 1 for the following description. The $68 \mathrm{HC} 11$ is run in expanded mode at a clock speed of $8 \mathrm{MHz}$. Input/Output ports $\mathrm{B}$ and $\mathrm{C}$ are used to interface to the external 27HC64 EPROM and the $62 \mathrm{HC} 64$ RAM chips. A 74HC373 multiplexer and a 74HC00 NAND GATE are used to form the proper address line controls and to transfer data to the external memory chips. Port PE0 is used for the input to the A/D converter. The logarithmically compressed signal passes through a low pass filter to remove any high frequency noise, and then is sequentially sampled by the A/D converter. Ports PD0 and PD1 are used for serial data output. A SCL/U $20 \mathrm{~mA}$ SAIL-to-low-power-logic converter is used to convert the $68 \mathrm{HC} 11$ 's serial data to the SAIL communication 
protocol. The use of the SAIL communications protocol is beneficial because of it's simplicity, and it's ability to communicate with multiple nodes. The rest of port D's output lines are tied to the positive supply through $100 \mathrm{k} \Omega$ pull up resistors to prevent the output lines from floating. Ports PA3, PA4, PA5, and PA7 are used to control the two CD4053 2 channel analog switches which control the attenuator network described earlier. The rest of port A's input/output lines, except PA0, are tied to ground through $100 \mathrm{k} \Omega$ resistors to prevent the outputs from floating. PA0 is left as an external input for further development, and is tied to the positive supply through a $100 \mathrm{k} \Omega$ pull up resistor.

Appendix 2 contains a listing of the program that the $68 \mathrm{HC} 11$ executes.

\subsubsection{Power Supply}

In the test receiver, $a+10$ volt power supply is used to power the preamplifier, the envelope detector, and the logarithmic compressor. A secondary +5 volt power supply is used to power the $68 \mathrm{HCl1}$ microcontroller. The use of a separate power supply for the microcontroller prevents high frequency noise generated by the digital components from entering the preamplifier. For the $-5 \mathrm{~V}$ that is required by the logarithmic compressor, a third supply is used. This provides a better regulated negative supply then using a DC to DC voltage converter such as the ICL 7660 to generate the required $-5 \mathrm{~V}$. Finally, a +12 volt, $300 \mathrm{~mA}$ supply is provided to power an external GPS receiver. All four power supplies are built on a common circuit board and share a common ground bus. Refer to the Power Supply schematic diagram in Appendix 1 for the following circuit description. 
The test receiver uses a standard power cord to connect to external power with a one half amp slow-blow fuse to protect the receiver in case of a short circuit. A Magnetek Triad F-93X transformer is used to step down the input voltage. Diodes $\mathrm{D}_{01}$ through $\mathrm{D}_{04}$ act as a standard full wave bridge rectifier. Voltage regulator $\mathrm{U}_{01}$ is a simple three terminal +12 volt regulator used to provide power to the external GPS receiver. Capacitor $\mathrm{C}_{01}$ filters the rectified supply voltage to provide an unregulated DC voltage to $\mathrm{U}_{01}$. Capacitors $\mathrm{C}_{02}$ and $\mathrm{C}_{03}$ are bypass capacitors used to reduce noise on $\mathrm{U}_{01}$ 's input and output leads. Because of the high current requirements of the voltage regulator, $\mathrm{U}_{01}$ is mounted to the chassis bottom for heat sinking.

Voltage regulator $\mathrm{U}_{02}$ is an adjustable four terminal voltage regulator used to provide the +10 volts required by the preamplifier, absolute value circuit, and the logarithmic compressor. Resistor $\mathrm{R}_{01}$ and capacitor $\mathrm{C}_{04}$ form a low pass filter which helps reduce high frequency feedback from the GPS receiver power supply $\left(U_{01}\right) . R_{01}$ also acts as an attenuator, reducing the unregulated DC input voltage to regulator $\mathrm{U}_{02}$. By doing this, the power that $\mathrm{U}_{02}$ has to sink is reduced, increasing $\mathrm{U}_{02}$ 's efficiency. Resistors $R_{02}, R_{03}$, and $R_{04}$ form the feedback network used by $U_{02}$ to set the output voltage. By using an adjustable voltage regulator, the positive supply voltage can be set more precisely, which is needed to maintain sensitive bias points in the logarithmic compressor. Capacitors $\mathrm{C}_{05}$ and $\mathrm{C}_{06}$ are bypass capacitors similar to $\mathrm{C}_{02}$ and $\mathrm{C}_{03}$.

Voltage regulator $\mathrm{U}_{03}$ is a simple three terminal regulator used to supply the +5 volts required by the $68 \mathrm{HCl1}$ and support systems. Resistor $\mathrm{R}_{05}$ and capacitor $\mathrm{C}_{07}$ form another low pass filter which helps isolate the preamplifier from the $68 \mathrm{HC} 11$ 
microcontroller. As with $\mathrm{R}_{01}, \mathrm{R}_{05}$ reduces the unregulated $\mathrm{DC}$ voltage at the input of $\mathrm{U}_{03}$, reducing the power that $\mathrm{U}_{03}$ must sink. Capacitors $\mathrm{C}_{08}$ and $\mathrm{C}_{09}$ are bypass capacitors similar to $\mathrm{C}_{02}$ and $\mathrm{C}_{03}$. The combination of resistor $\mathrm{R}_{06}$ and LED $\mathrm{D}_{05}$ are used as a power indicator, with $\mathrm{D}_{0 \text { s }}$ mounted on the front panel of the chassis next to the power switch.

Finally, voltage regulator $\mathrm{U}_{04}$ is a four terminal, adjustable negative voltage regulator that is used to provide the -5 volts required for the logarithmic compressor. Resistor $\mathrm{R}_{07}$ and capacitor $\mathrm{C}_{10}$ form a low pass filter which converts the rectified input voltage to unregulated DC. Resistors $R_{08}, R_{09}$, and $R_{10}$ form the feedback network used by $\mathrm{U}_{04}$ to set the output voltage at -5 volts. As before, $\mathrm{C}_{11}$ and $\mathrm{C}_{12}$ are bypass capacitors that help reduce noise the voltage regulator's input and output lines.

\subsection{Test Receiver Performance}

To test the validity of the receiver designed in section 5.4, experiments were conducted to measure the actual performance of the preamplifier, envelope detector, and logarithmic compressor.

\subsubsection{Preamplifier Performance}

To test the performance of the preamplifier, experiments were conducted to measure the preamplifier's actual gain, bandwidth, and thermal noise. To measure the gain and bandwidth of the preamplifier, the output of the preamplifier was measured for sinusoidal inputs at different frequencies. The results, along with the predicted values are plotted in figure 5-8. From figure 5-8 we see that the actual gain of the preamplifier at the acoustic beacon's carrier frequency is $86 \mathrm{~dB}$. This is close enough 


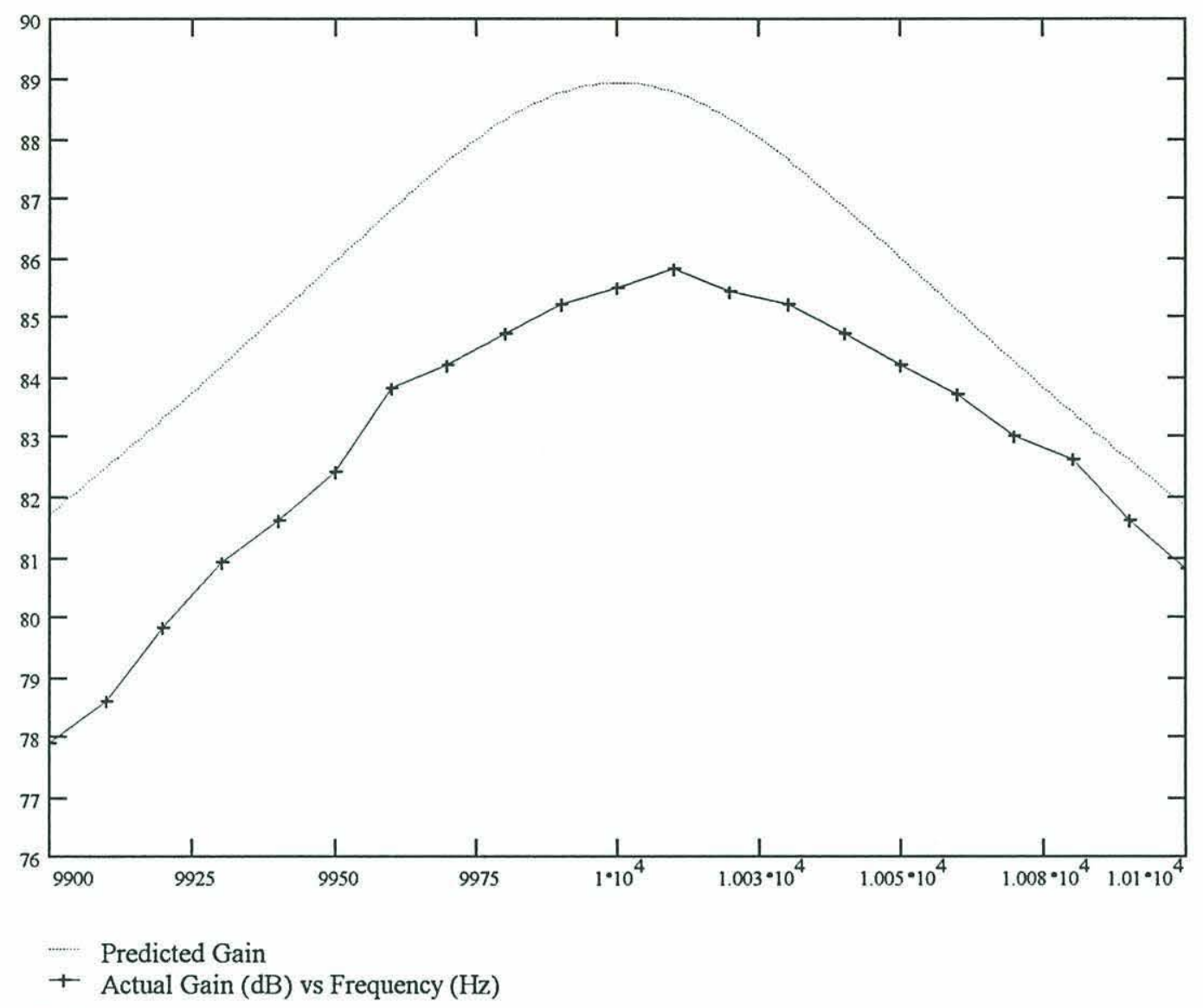

Figure 5-8

to the design goal to be acceptable. The loss in gain compared to the predicted gain is due to the effects of loading between the various stages and to deviations from the nominal values of $Y_{\mathrm{fs}}$ and $\mathrm{r}_{\mathrm{d}}$. We also note that the actual bandwidth is $120 \mathrm{~Hz}$ which is slightly larger then the predicted bandwidth. This increase is due to deviations of $R_{02}$ and $R_{11}$ from their nominal values and is not significant.

To determine the actual thermal noise of the preamplifier, we short the preamplifier's input and measure the root mean square output using a digital multimeter. Assuming the preamplifier's noise is normally distributed, we correct the 
output measurement to reflect a normal distribution by multiplying by 1.13 [Horowitz and Hill 1989]. The preamplifier's thermal noise is then found by using:

$$
e_{n}=\frac{1.13 \cdot e_{s}}{A_{v} \sqrt{B W}}
$$

where $e_{s}$ is the measured output voltage, $A_{v}$ is the total gain of the preamplifier, and $\mathrm{BW}$ is the bandwidth of the preamplifier. Using this approach, $\mathrm{e}_{\mathrm{s}}$ was measured as 6.8 millivolts. The preamplifier's thermal noise is then:

$$
e_{n} \approx \frac{1.13 \cdot 6.8 \cdot 10^{-3}}{19275 \sqrt{120}} \approx 31 \mathrm{nV} / \sqrt{\mathrm{Hz}}
$$

To check this value we can observe the output of the preamplifier (with the input grounded) on an oscilloscope. To measure the preamplifier's noise, we take the peak value observed on the oscilloscope. Next we calculate the preamplifier's thermal noise using:

$$
e_{n}=\frac{V_{\text {peak }} / 6}{A_{v} \sqrt{B W}}
$$

This gives a rough approximation of the thermal noise of the preamplifier. $\mathrm{V}_{\text {peak }}$ was measured as $35 \mathrm{mV}$. Using equation (5-31), the thermal noise of the preamplifier is roughly $28 \mathrm{nV} / \sqrt{\mathrm{Hz}}$. This value is of the same order of magnitude as found in equation (5-30), so we can assume the noise figure given in equation (5-30) is correct.

\subsubsection{Envelope Detector}

To test the performance of the envelope detector we look at the output for 
different amplitudes of a sinusoidal input. Figure 5-9 shows the measured output of the envelope detector for different amplitudes of a 10,000 Hertz sinusoid input.

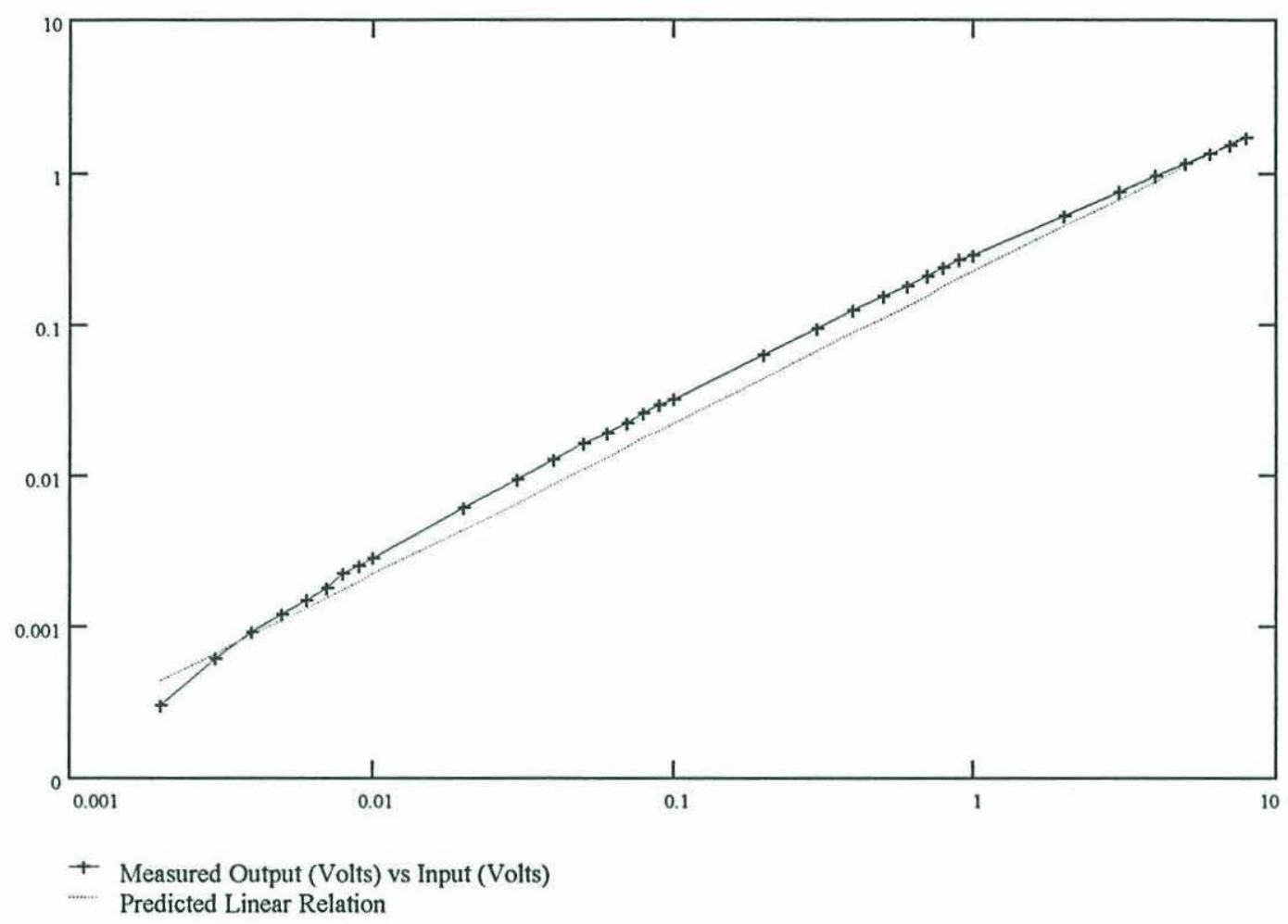

Figure 5-9

Ideally, the envelope detector should have an output that is linearly dependent on the amplitude of the input sinusoid. This allows the envelope detector to accurately track the true envelope of the beacon signal. From figure 5-9 we see that the measured output is nearly linear, therefore we expect the envelope detector to work properly.

\subsubsection{Logarithmic Compressor}

To test the performance of the logarithmic compressor, we look at the output for different DC voltage inputs. Figure 5-10 shows the measured output values and the predicted values. 


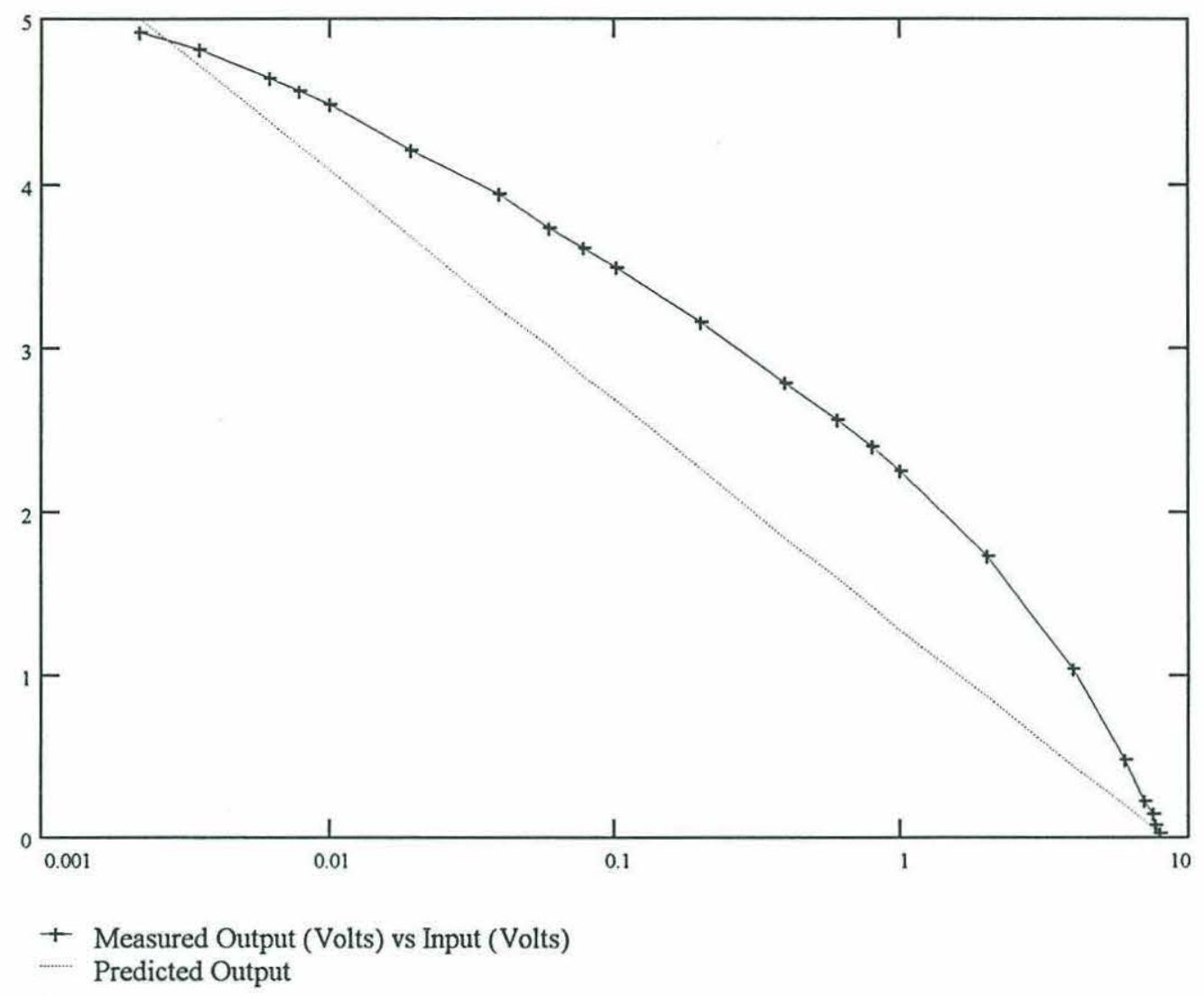

Figure 5-10

From figure 10 we see that the logarithmic compressor does not follow an exact logarithmic relation. However, since the output of the logarithmic compressor monotonically decreases throughout the full range of input values, the compressor is sufficiently logarithmic to achieve the dynamic resolution of equation (5-28). Since we achieve the desired dynamic resolution, the performance of the logarithmic compressor is acceptable.

\subsection{Conclusions}

In this chapter a receiver to implement the maximum likelihood estimation algorithms developed in chapter II and III was designed and tested. Appendix 1 
contains the schematic diagrams of the receiver built for testing. From the tests conducted, we see that the test receiver meets the design criteria developed in section 5.2. To complete the receiver, an Apple Macintosh personal computer is used for the final signal processing and for data display. Appendix 3 contains a listing of the source code used by the Macintosh for signal processing and for displaying the collected data. 


\section{Chapter VI}

\section{Conclusion}

\subsection{Observations and Summary}

In this thesis, a method to passively localize stationary and linearly moving acoustic beacons was developed. Because of the nonlinearities of the structural model $g\left(\mathbf{w}_{\mu}, \theta\right)$, and the errors in the measurements $x_{\mu}, y_{\mu}$, and $t_{s \mu}$, the localization problem was formulated as a constrained maximum likelihood estimation problem. To solve for the maximum likelihood estimate, a vector of Lagrange multipliers was introduced and a Lagrangian function $\Lambda$ formed. The maximum likelihood estimate was then found at the stationary point of the Lagrangian function. Figures 2-4 and 3-1 summarize the algorithms used to solve for the maximum likelihood estimate of the acoustic beacon's position. Both algorithms require that the user specify the maximum range and depth of the search area, and an initial guess of the acoustic beacon's location. For the case of the moving beacon, the user must also specify the maximum allowed velocity of the acoustic beacon.

Monte Carlo simulations were conducted for several different positions and velocities of the acoustic beacon. It was assumed that the receiving ship followed a hexagonal search path with legs of 1000 meters. In practice, the actual search path is not critical as long as it is not linear. In chapter IV results from six scenarios were presented. Overall, the algorithms developed in chapters II and III performed well for these scenarios. For a stationary acoustic beacon, good estimates of the acoustic beacon's location were obtain using GPS errors of 3 meters and 25 meters rms. For 
the moving acoustic beacon, a good estimate of the acoustic beacon's location was obtained when using GPS errors of 3 meters rms. However, the accuracy of the algorithm used to estimate the moving acoustic beacon's location degrades for GPS errors of 25 meters rms unless a large number of experiment vectors are used, or a large effective sensor spacing is used.

From the results of the Monte Carlo simulations, it was shown that, in general, as the number of experiment vectors $\left(\mathrm{w}_{\mu}\right)$ used in the maximum likelihood estimation problem increased, or the effective sensor spacing increased, the accuracy of the estimated beacon location improved. It was also shown that the accuracy of the estimated beacon location improved if the receiving ship passed through a closest point of approach to the acoustic beacon. In all cases, the accuracy of the estimated beacon position was significantly better when using 3 meter GPS errors. This shows that to obtain a better estimate of the acoustic beacon's location, the position of the receiving ship must be measured accurately.

For all scenarios, the variance of the estimated beacon position and velocity decreased as the number of experiment vectors used increased, or the effective sensor spacing increased. It was found that the variance of the moving beacon's estimated position was much greater then the variance of the stationary beacon's estimated position. This is due the manner in which the acoustic beacon's initial position and velocity are combined in the modified structural model. In the modified structural model given by equation $3-10$, the individual velocity components are added to the initial beacon coordinates $\mathrm{x}_{0}, \mathrm{y}_{0}$, and $\mathrm{z}_{\mathrm{o}}$ to find the location of the acoustic beacon 
when it transmits a signal. As a result, the algorithm estimates the value of the sum of the initial position coordinates and the velocities better then the individual components. Consequently, the algorithm can not discern between small perturbations in the estimated position coordinates and the estimated velocities.

From these results, to obtain the best possible estimate of the acoustic beacon's position a large number of experiment vectors with a large effective sensor spacing must be used. However, the time required to collect the measurement vectors increases with both the number of experiment vectors and the effective sensor spacing. If the time it takes to obtain an estimate of the acoustic beacon's position is an issue, fewer experiment vectors and smaller effective sensor spacings can be used. For the hexagonal search path shown in figure 4-1, and a pulse repetition interval of 2 seconds, a minimum of 20 experiment vectors with an effective sensor spacing of 35 pulse repetition intervals are required to obtain a decent estimate of the acoustic beacon's position for a stationary beacon. For the moving beacon, assuming the same search path, a minimum of 30 experiment vectors with an effective sensor spacing of 45 pulse repetition intervals are required.

In chapter $\mathrm{V}$ design specifications for a receiver to implement the algorithms given in chapters II and III were developed. The synchronous time of receipt was measured by determining the time of arrival of the acoustic beacon's envelope. To determine the arrival time of the acoustic beacon's envelop, the beacon's carrier frequency was first removed using a precision rectifier with a low pass filter to form an envelope detector. The output of the envelope detector was then logarithmically 
compressed to increase the dynamic resolution of the receiver. Next, the output of the logarithmic compressor was digitally sampled using a $68 \mathrm{HC} 11$ microcontroller. Following A/D conversion, the sampled envelope was passed through a digital matched filtered. For a rectangular windowing function, this produces a unique maximum output which was used to determine the time of arrival. Following the design specifications, a receiver was built from commonly available components. Experiments conducted on receiver showed that the test receiver met the design specifications of section 5.2. 


\section{Appendix 1}

This appendix contains the schematic diagrams for the receiver designed in chapter V. 


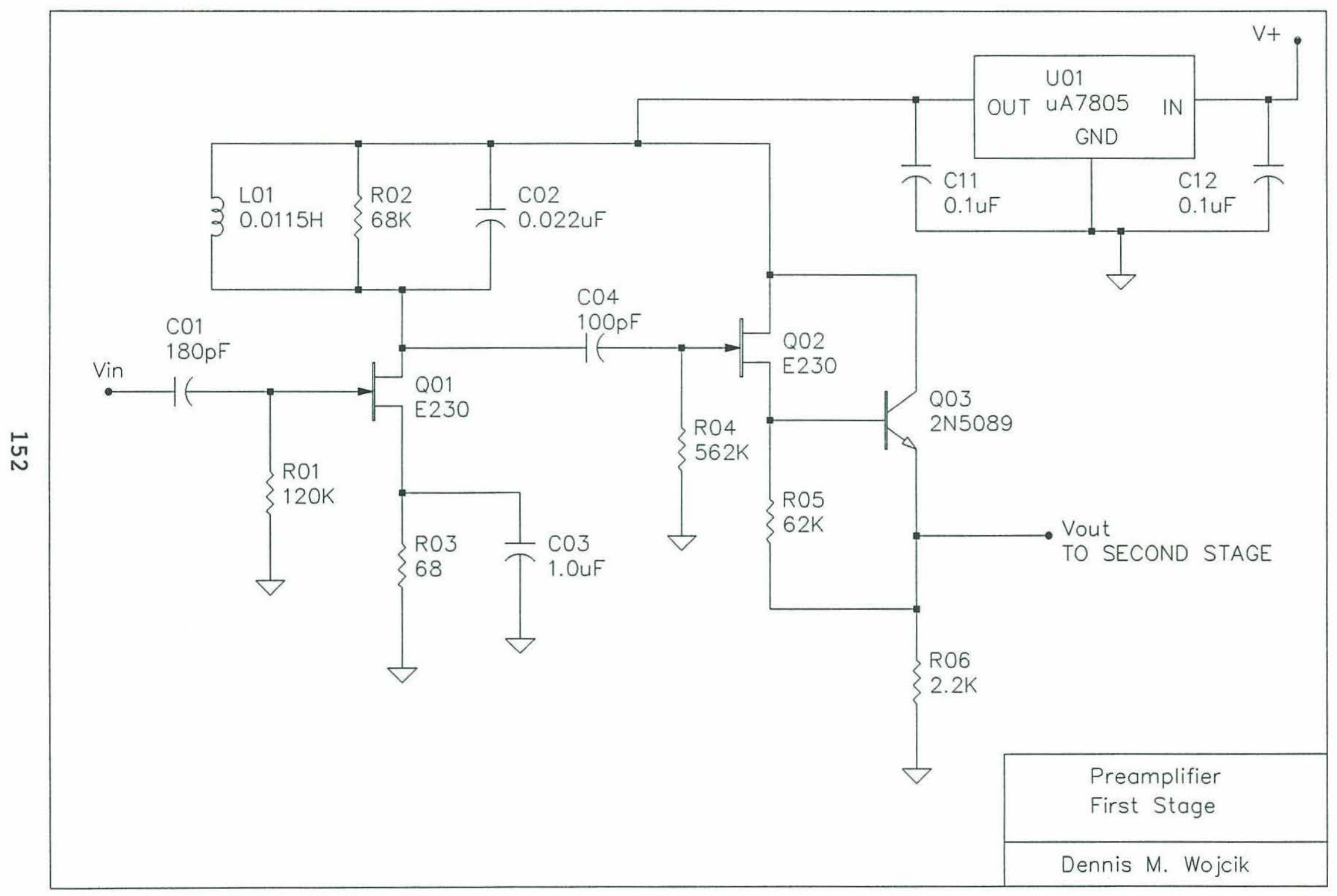




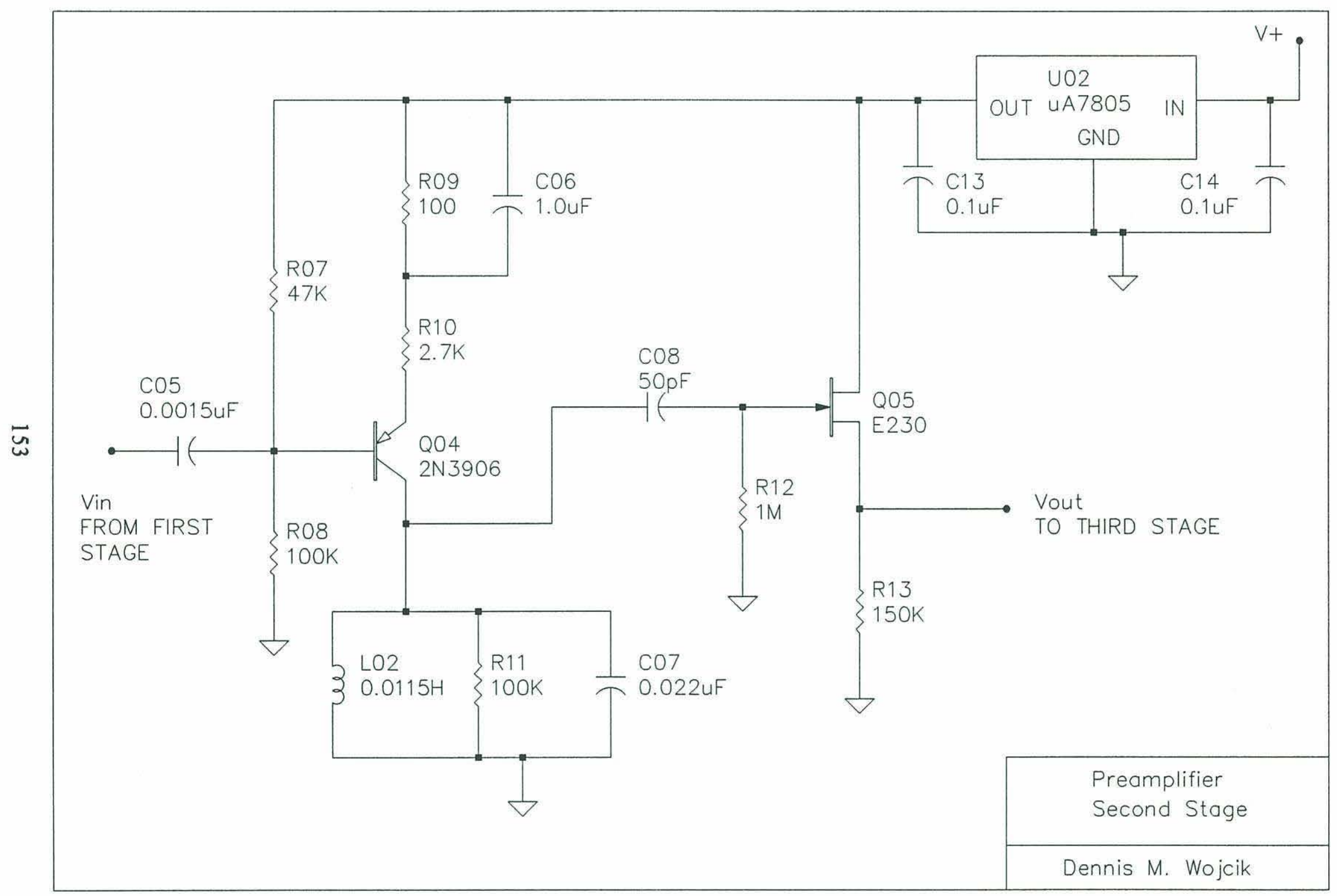




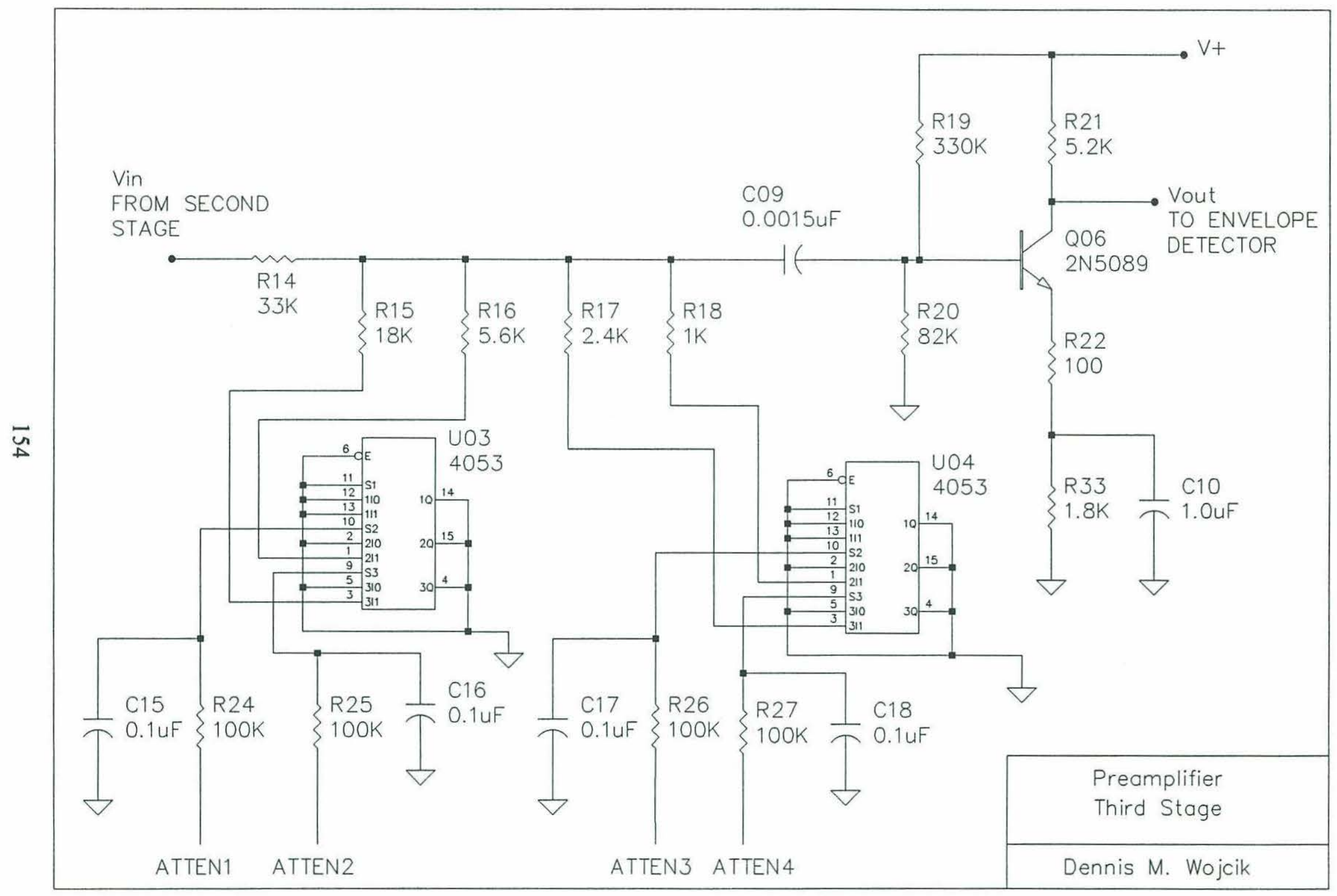




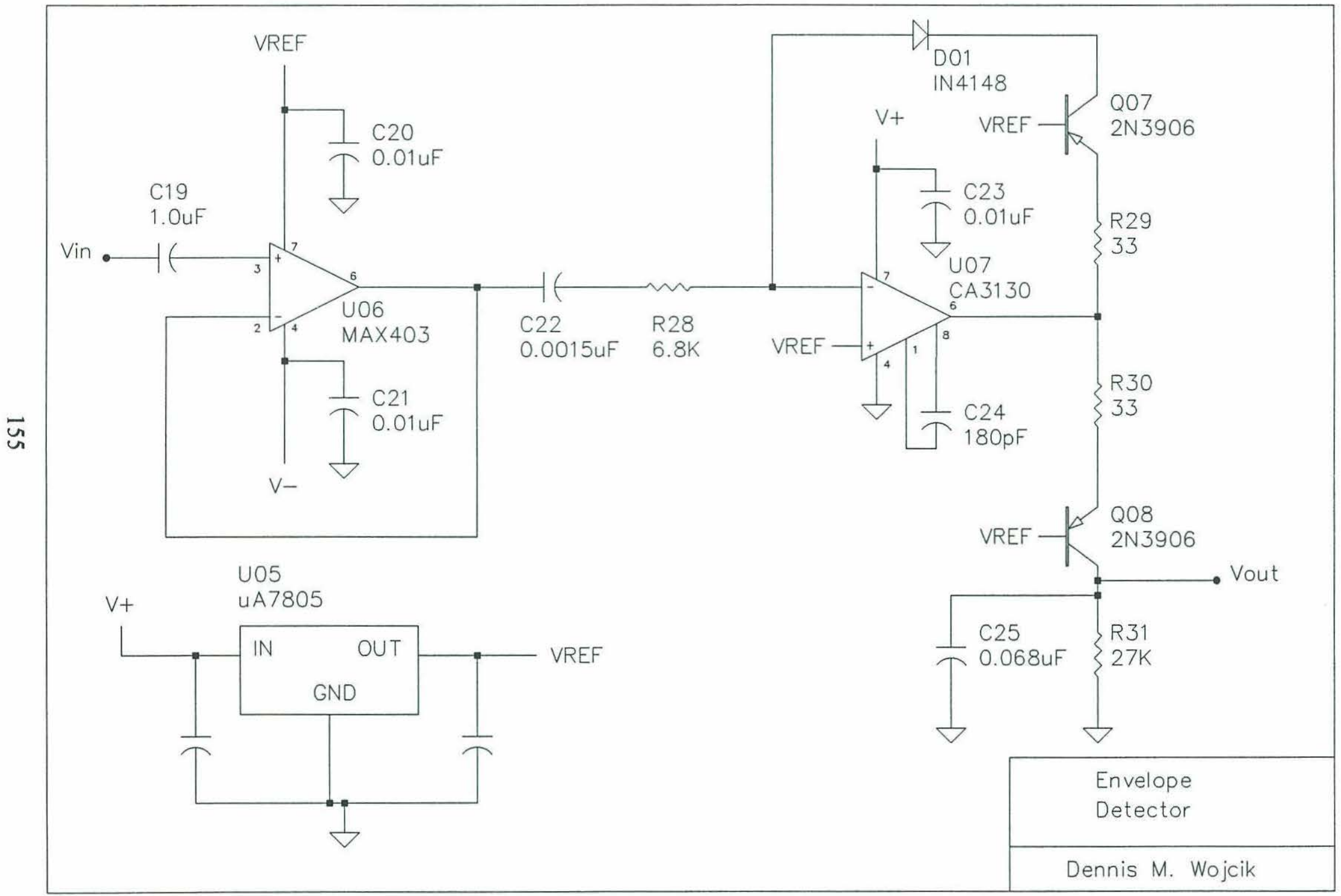




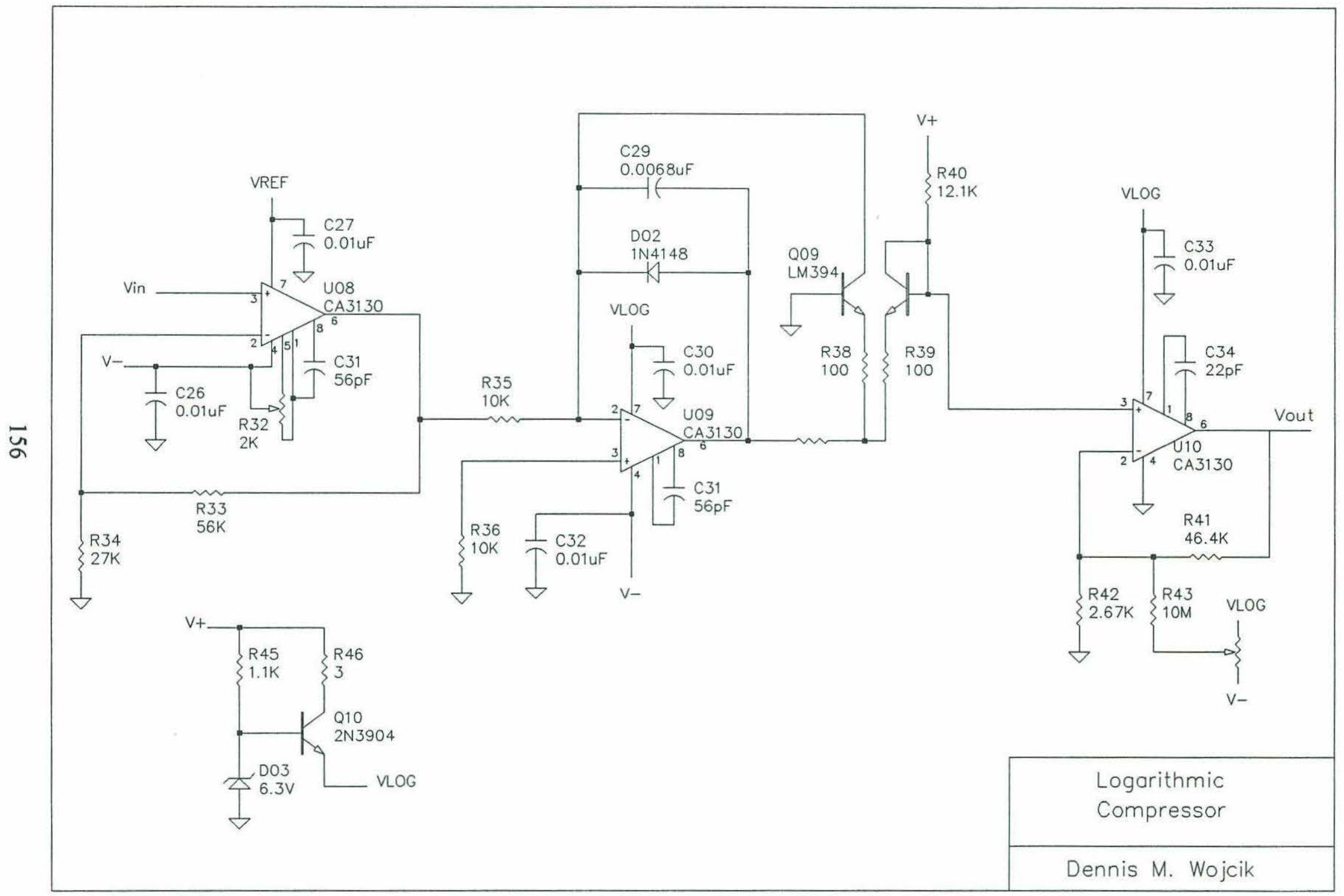




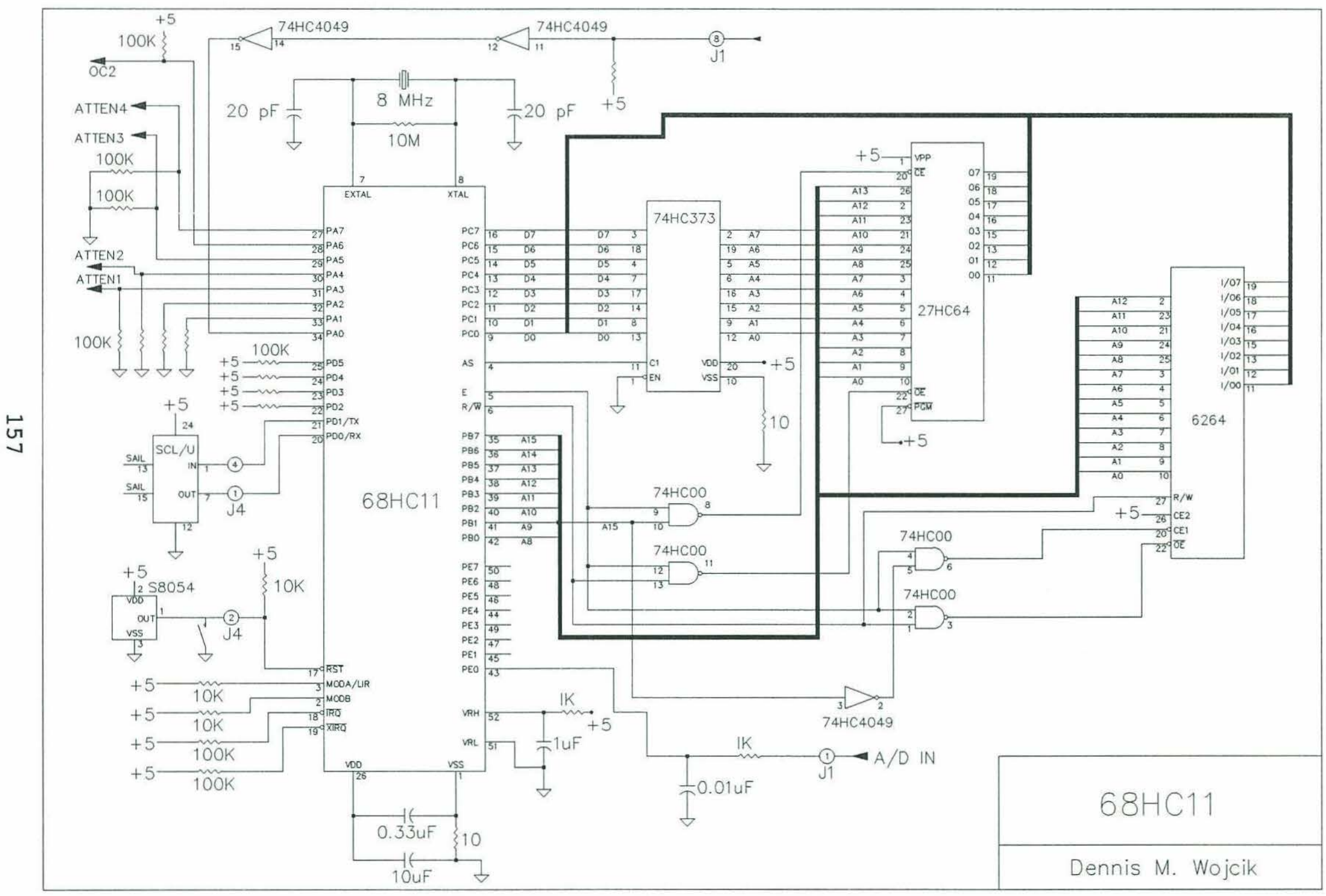




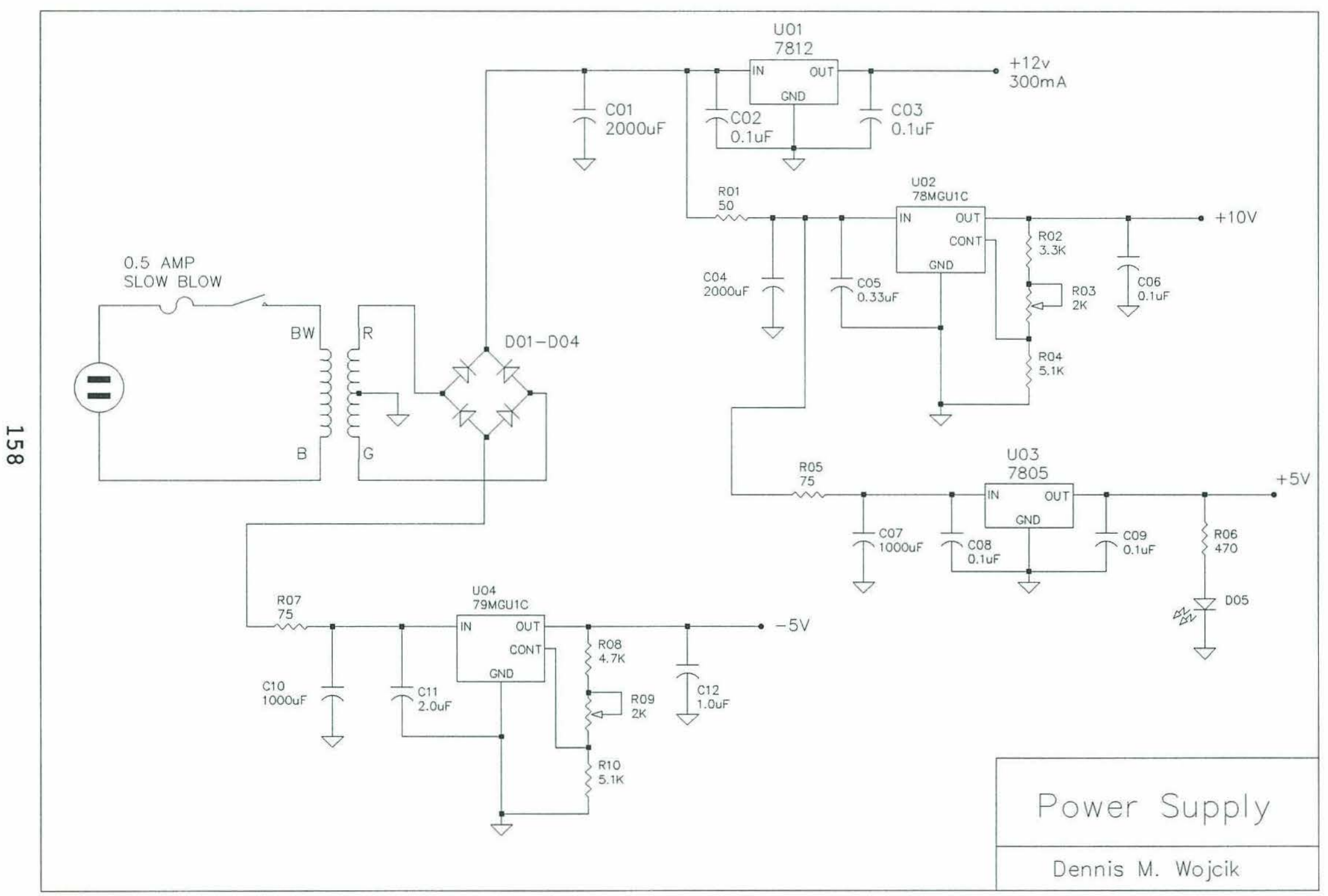




\section{Appendix 2}

This appendix contains the source code used by the $68 \mathrm{HC} 11$. The code is based on a full function SAIL driver written by Dr. Albert Bradley and operates under serial communication interrupt control. The sampling rate of the A/D converter is set by using a Timer Output Compare function. After the microcontroller finishes an initialization period, it sits in a wait state until a serial communications interrupt or a timer output compare interrupt occurs. Once the interrupt is received, the microcontroller executes the requested task, and then returns to the wait state.

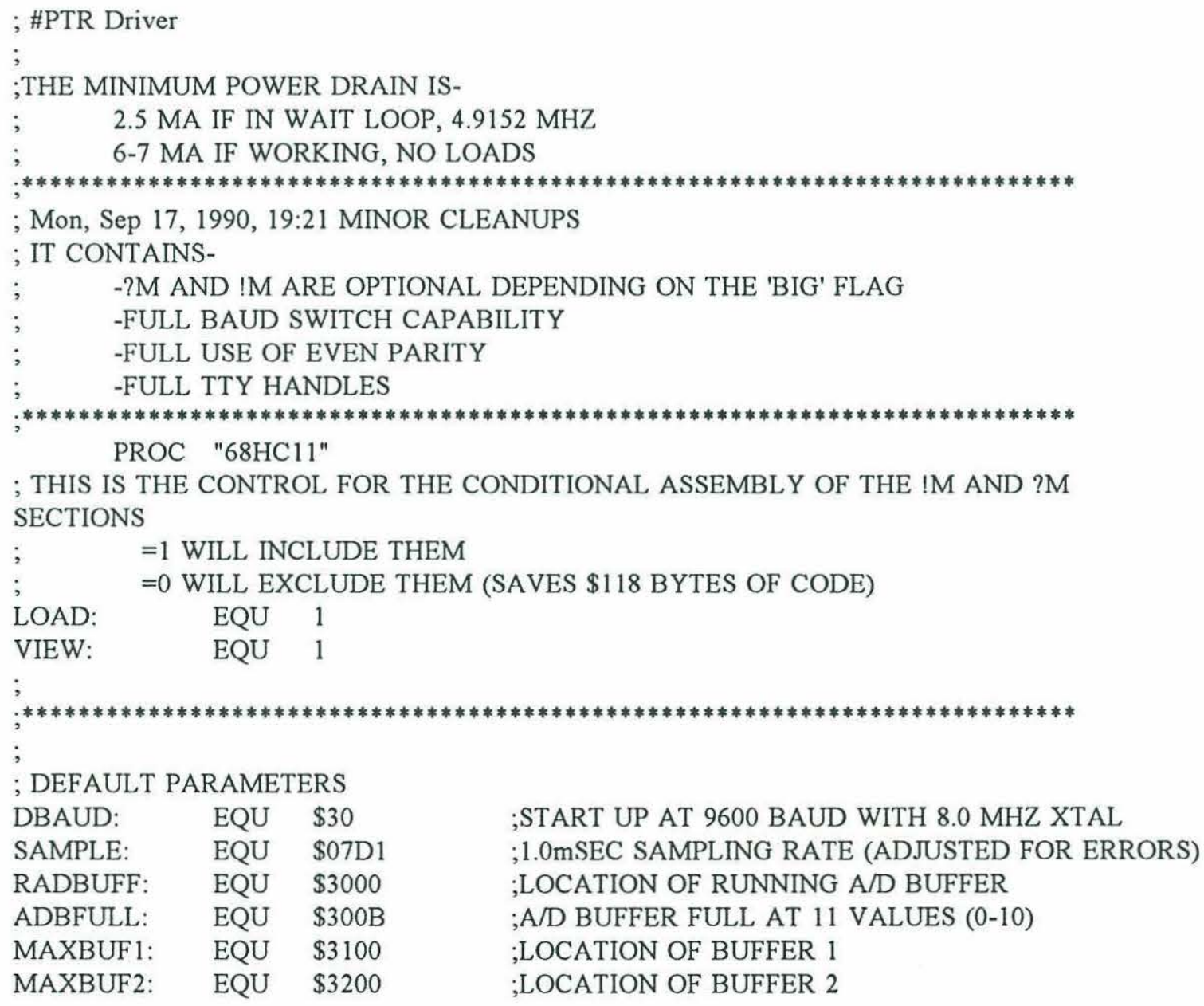

;START UP AT 9600 BAUD WITH 8.0 MHZ XTAL ;1.0mSEC SAMPLING RATE (ADJUSTED FOR ERRORS) ;LOCATION OF RUNNING A/D BUFFER ;A/D BUFFER FULL AT 11 VALUES (0-10) ;LOCATION OF BUFFER 1 ;LOCATION OF BUFFER 2 


\begin{tabular}{|c|c|c|c|}
\hline ATTNINC: & EQU & \$E8 & ;INCREASE ATTN AT 4.75 VOLTS \\
\hline ATTNDEC: & EQU & $\$ 40$ & ;INCREASE THE PREAMP GAIN AT THIS LEVEL \\
\hline MAX1FL: & EQU & $\$ 310 \mathrm{~F}$ & ;CHECK VALUE TO INITIALIZE MAX BUFFER \\
\hline MAX2FL: & EQU & $\$ 320 \mathrm{~F}$ & ;CHECK VALUE TO INITIALIZE MAX BUFFER \\
\hline \multirow{2}{*}{\multicolumn{4}{|c|}{; ATTENUATOR LEVELS }} \\
\hline & & & \\
\hline DB00: & EQU & $\$ 01$ & ; 0 DB ATTEN \\
\hline DB03: & EQU & $\$ 09$ & ; 3 DB ATTEN \\
\hline DB09: & EQU & $\$ 11$ & ; 9 DB ATTEN \\
\hline DB12: & EQU & $\$ 19$ & ;12 DB ATTEN \\
\hline DB18: & EQU & $\$ 21$ & ;18 DB ATTEN \\
\hline DB24: & EQU & $\$ 81$ & ;24 DB ATTEN \\
\hline DB30: & EQU & \$B9 & ;30 DB ATTEN \\
\hline \\
\hline DMAXNUM: & EQU & $\$ 02$ & ;DEFAULT NUMBER OF MAX POINTS TAKEN \\
\hline DPERIOD: & EQU & \$07D0 & ;DEFAULT TO 2 SECOND PERIOD \\
\hline DWIND: & EQU & $\$ 0014$ & ;DEFAULT INDEX WINDOW \\
\hline \multirow{2}{*}{\multicolumn{4}{|c|}{ 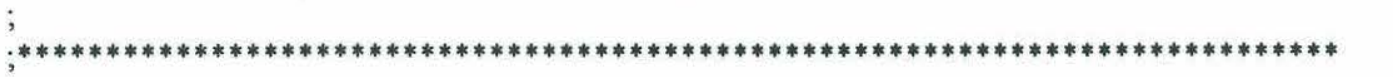 }} \\
\hline & & & \\
\hline \\
\hline PORTA: & EQU & $\mathrm{RP}+\$ 00$ & ;|OC1.OC2.OC3.OC4!OC5.IC1.IC2.IC3| \\
\hline PORTC: & EQU & $\mathrm{RP}+\$ 03$ & ; \\
\hline PORTB: & EQU & $\mathrm{RP}+\$ 04$ & ; \\
\hline DDRC: & EQU & $\mathrm{RP}+\$ 07$ & ; \\
\hline PORTD: & EQU & $\mathrm{RP}+\$ 08$ & ;X.X.SS.SCK!MOSI.MISO.TXD.RXD| \\
\hline DDRD: & EQU & $\mathrm{RP}+\$ 09$ & 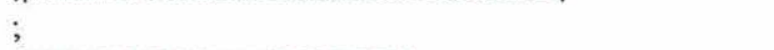 \\
\hline PORTE: & EQU & $\mathrm{RP}+\$ 0 \mathrm{~A}$ & ;|V7.V6.V5.V4!X.X.X.BRK| \\
\hline \multicolumn{4}{|r|}{;BAT.BAUD. } \\
\hline CFORC: & EQU & $\mathrm{RP}+\$ 0 \mathrm{~B}$ & ;TIMER COMPARE FORCE REGISTER \\
\hline OC1M: & EQU & $\mathrm{RP}+\$ 0 \mathrm{C}$ & ;OUT COMP 1 MASK REG \\
\hline OC1D: & EQU & $\mathrm{RP}+\$ 0 \mathrm{D}$ & ;OUT COMP 1 DATA REG \\
\hline TCNT: & EQU & $\mathrm{RP}+\$ 0 \mathrm{E}$ & ;TIMER REGISTER (HIGH BYTE) \\
\hline DTIC1: & EQU & $\mathrm{RP}+\$ 10$ & ;TIMER INPUT CAPTURE 1 (HI BYTE) \\
\hline DTIC2: & EQU & $\mathrm{RP}+\$ 12$ & ;TIMER INPUT CAPTURE 2 (HI BYTE) \\
\hline DTIC3: & EQU & $\mathrm{RP}+\$ 14$ & ;TIMER INPUT CAPTURE 3 (HI BYTE) \\
\hline DTOC1: & EQU & $R P+\$ 16$ & ;TIMER OUTPUT COMPARE 1 (HI BYTE) \\
\hline DTOC2: & EQU & $\mathrm{RP}+\$ 18$ & ;TIMER OUTPUT COMPARE 2 (HI BYTE) \\
\hline DTOC3: & EQU & $\mathrm{RP}+\$ 1 \mathrm{~A}$ & ;TIMER OUTPUT COMPARE 3 (HI BYTE) \\
\hline DTOC4: & EQU & $\mathrm{RP}+\$ 1 \mathrm{C}$ & ;TIMER OUTPUT COMPARE 4 (HI BYTE) \\
\hline DTOC5: & EQU & $\mathrm{RP}+\$ 1 \mathrm{E}$ & ;TIMER OUTPUT COMPARE 5 (HI BYTE) \\
\hline TCTL1: & EQU & $R P+\$ 20$ & ;TIMER CONTROL REG 1 \\
\hline TCTL2: & EQU & $\mathrm{RP}+\$ 21$ & ;TIMER CONTROL REG 2 \\
\hline TMSK1: & EQU & $\mathrm{RP}+\$ 22$ & ;TIMER INTERRUPT MASK REG 1 \\
\hline TFLG1: & EQU & $\mathrm{RP}+\$ 23$ & ;TIMER INTERRUPT FLAG REG 1 \\
\hline TMSK2: & EQU & $\mathrm{RP}+\$ 24$ & ;TIMER INTERRUPT MASK REG 2 \\
\hline TFLG2: & EQU & $\mathrm{RP}+\$ 25$ & ;TIMER INTERRUPT FLAG REG 2 \\
\hline PACTL: & EQU & $\mathrm{RP}+\$ 26$ & ;PULSE ACCUMULATOR CONTROL REG \\
\hline PACNT: & EQU & $R P+\$ 27$ & ;PULSE ACCUMULATOR COUNT REG \\
\hline SPCR: & EQU & $\mathrm{RP}+\$ 28$ & ;SPI CONTROL REGISTER \\
\hline SPSR: & EQU & $R P+\$ 29$ & ;SPI STATUS REGISTER \\
\hline
\end{tabular}




\begin{tabular}{|c|c|c|c|}
\hline SPDR: & EQU & $\mathrm{RP}+\$ 2 \mathrm{~A}$ & ;SPI DATA REGISTER \\
\hline BAUD: & EQU & $\mathrm{RP}+\$ 2 \mathrm{~B}$ & \\
\hline SCCR1: & EQU & $\mathrm{RP}+\$ 2 \mathrm{C}$ & \\
\hline SCCR2: & EQU & $R P+\$ 2 D$ & \\
\hline SCSR: & EQU & $R P+\$ 2 E$ & \\
\hline SCDR: & EQU & $\mathrm{RP}+\$ 2 \mathrm{~F}$ & \\
\hline ADCTL: & EQU & $\mathrm{RP}+\$ 30$ & \\
\hline ADR1: & EQU & $\mathrm{RP}+\$ 31$ & \\
\hline ADR2: & EQU & $\mathrm{RP}+\$ 32$ & ; \\
\hline ADR3: & EQU & $R P+\$ 33$ & ; \\
\hline ADR4: & EQU & $\mathrm{RP}+\$ 34$ & ; \\
\hline OPTION: & EQU & $\mathrm{RP}+\$ 39$ & \\
\hline HPRIO: & EQU & $\mathrm{RP}+\$ 3 \mathrm{C}$ & \\
\hline INIT: & EQU & $\mathrm{RP}+\$ 3 \mathrm{D}$ & ;[RAM3.2.1.0!REG3.2.1.0] \\
\hline \multicolumn{4}{|r|}{ 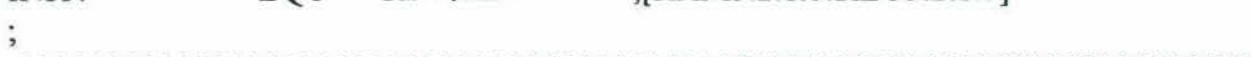 } \\
\hline \multicolumn{4}{|c|}{; SAIL inteface RAM workspace } \\
\hline RJUMP: & EQU & $\$ 40$ & ; JUMP INST GOES HERE.. \\
\hline RVECT: & EQU & $\$ 41$ & HI \\
\hline ; & & $\$ 42$ & LO \\
\hline ХЛUMP: & EQU & $\$ 43$ & ;XMIT JUMP INST \\
\hline XVECT: & EQU & $\$ 44$ & ; $\quad \mathrm{HI}$ \\
\hline ; & & $\$ 45$ & LO \\
\hline JUMP2: & EQU & $\$ 46$ & ;2ND JUMP INST \\
\hline VECT2: & EQU & $\$ 47$ & ; $\quad$ HI \\
\hline ; & & $\$ 48$ & LO \\
\hline SCRTCH1: & EQU & $\$ 49$ & ;SCRATCH LOC 1 \\
\hline SCRTCH2: & $\mathrm{EQU}$ & $\$ 4 \mathrm{~A}$ & ;SCRATCH LOC 2 \\
\hline SCRTCH3: & EQU & $\$ 4 \mathrm{~B}$ & ;SCRATCH LOC 3 \\
\hline SCRTCH4: & EQU & $\$ 4 \mathrm{C}$ & ;SCRATCH LOC 4 \\
\hline SCRTCH5: & EQU & $\$ 4 \mathrm{D}$ & ;SCRATCH LOC 5 \\
\hline ; & & & \\
\hline XTTY: & EQU & $\$ 4 \mathrm{E}$ & ;TTY POINTER HI \\
\hline ; & & $\$ 4 \mathrm{~F}$ & LOW \\
\hline \multirow{2}{*}{\multicolumn{4}{|c|}{ 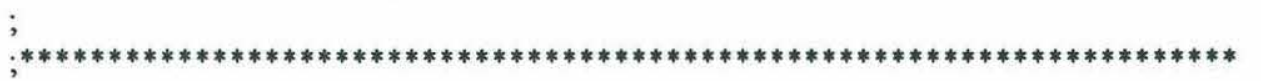 }} \\
\hline & & & \\
\hline \multicolumn{4}{|c|}{; PTR Data inteface RAM workspace } \\
\hline PERINDX: & EQU & $\$ 50$ & ;PERIOD TIMER INDEX HI \\
\hline ; & & $\$ 51$ & LOW \\
\hline PERIOD: & EQU & $\$ 52$ & ;LENGTH OF PERIOD HI \\
\hline ; & & $\$ 53$ & LOW \\
\hline MAXBUFF: & EQU & $\$ 54$ & ;MAXIMUMS BUFFER LOCATION HI \\
\hline ; & & $\$ 55$ & LOW \\
\hline MAXFULL: & EQU & $\$ 56$ & ;MAX BUFFER FULL LOCATION HI \\
\hline ; & & $\$ 57$ & LOW \\
\hline ADBFPTR: & EQU & $\$ 58$ & ;RUNNING A/D BUFFER LOCATION HI \\
\hline ; & & $\$ 59$ & LOW \\
\hline TXBUFF: & EQU & $\$ 5 \mathrm{~A}$ & ;MAXIMUMS BUFFER TO TX HI \\
\hline ; & & $\$ 5 B$ & LOW \\
\hline TXBFULL: & EQU & $\$ 5 \mathrm{C}$ & ;TX BUFFER FULL LOCATION HI \\
\hline
\end{tabular}




\begin{tabular}{|c|c|c|c|}
\hline \multirow{3}{*}{$\begin{array}{l}\text {; } \\
\text { TX1FLG: } \\
\text { TX2FLG: }\end{array}$} & & $\$ 5 \mathrm{D}$ & LOW \\
\hline & EQU & $\$ 5 \mathrm{E}$ & ;FIRST MAXIMUMS SENT FLAG \\
\hline & EQU & $\$ 5 \mathrm{~F}$ & \\
\hline MAXFLG: & EQU & $\$ 60$ & ;WHICH MAXIMUM BUFFER TO SAVE FLAG \\
\hline AGCFLG: & EQU & $\$ 61$ & ;MANUAL GAIN CONTROL FLAG \\
\hline BIG: & EQU & $\$ 62$ & ;LARGEST A/D VALUE PER PERIOD LOCATION \\
\hline MAXNUM: & EQU & $\$ 63$ & ;NUMBER OF MAXIMUMS LOCATION \\
\hline TXMHI: & EQU & $\$ 64$ & ;TRANSMIT OUT BUFFERS \\
\hline TXMLO: & EQU & $\$ 65$ & \\
\hline TXIHI: & EQU & $\$ 66$ & \\
\hline TXILO: & EQU & $\$ 67$ & \\
\hline TXINT: & EQU & $\$ 68$ & ;INTERM STORAGE FOR TX POINTER \\
\hline TEMPIND: & EQU & $\$ 70$ & ;INTERM STORAGE FOR MAXIMUM INDEX \\
\hline TEMPMAX: & EQU & $\$ 72$ & ;INTERM STORAGE FOR MAXIMUM \\
\hline WINDOW: & EQU & $\$ 74$ & ;MAXIMUM CHECK WINDOW SIZE \\
\hline WINCHK: & EQU & $\$ 76$ & ;USED FOR TESTING IF WITHIN WINDOW \\
\hline INDXCHK: & EQU & $\$ 78$ & 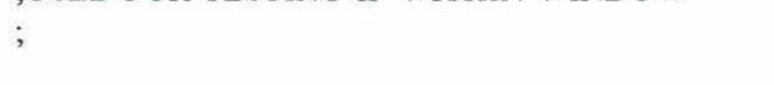 \\
\hline \multicolumn{4}{|c|}{ 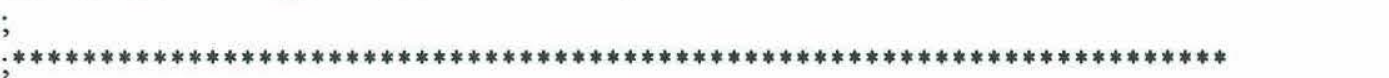 } \\
\hline \multirow{2}{*}{\multicolumn{4}{|c|}{$\begin{array}{l}\text { ORG \$E800 ;ASSUME 27C64 EPROM } \\
\text {; SAIL Address stored here.. }\end{array}$}} \\
\hline & & iere.. & \\
\hline \multirow[t]{2}{*}{ ADDR: } & FCB & "\#PTR" & ;SAIL ADDRESS \\
\hline & FCB & $\$ 00$ & ;TERMINATOR \\
\hline \\
\hline \multirow[t]{8}{*}{ START: } & SEI & & \\
\hline & CLR & $\$ 103 \mathrm{D}$ & ;MOVE REGISTERS TO BASE PAGE $(00->$ INIT) \\
\hline & LDS & \#\$OOFF & ;SET STACK POINTER \\
\hline & LDAA & \#DBAUD & ;SET DEFAULT BAUD \\
\hline & STAA & BAUD & \\
\hline & CLR & SCCR1 & ;8 DATA BITS, WAKE ON IDLE? \\
\hline & LDAA & $\# \$ 2 \mathrm{C}$ & ;ONLY REC INTERRUPTS YET \\
\hline & STAA & SCCR2 & \\
\hline \multicolumn{4}{|c|}{ 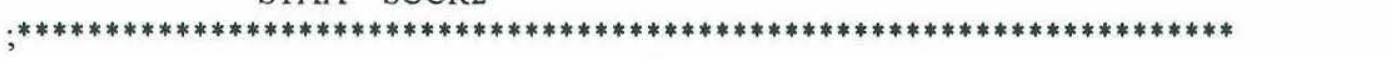 } \\
\hline \multicolumn{4}{|c|}{; PTR TIMER INTERUPT INITIALIZATION } \\
\hline & LDAA & $\# \$ 40$ & ;TOGGLE OC2 ON EACH COMPARE \\
\hline & STAA & TCTL1 & \\
\hline & CLR & TMSK2 & ;SET PRESCALE TO /1 \\
\hline & LDAA & $\# \$ 90$ & ;TURN ON THE A/D CONVERTER \\
\hline & STAA & OPTION & \\
\hline & LDAA & $\# \$ 20$ & ;USE PEO FOR A/D INPUT, SINGLE CHANNEL, \\
\hline & STAA & ADCTL & ;CONTINUOUS SAMPLING \\
\hline & LDAA & $\# \$ 88$ & ;SET PORT A PINS 7 AS OUTPUT \\
\hline & STAA & PACTL & \\
\hline & CLR & MAXFLG & ;INITIALIZE TO FIRST MAXIMUMS BUFFER \\
\hline & CLR & TX1FLG & ;INITIALIZE TO NOT TX'D \\
\hline & CLR & TX2FLG & ;INITIALIZE TO NOT TX'D \\
\hline & CLR & AGCFLG & ;INITIALIZE TO AGC ON \\
\hline & CLR & BIG & ;INITIALIZE THE LARGEST VALUE TO ZERO. \\
\hline & CLR & TXMHI & ;CLEAR THE OUTGOING BUFFERS \\
\hline
\end{tabular}




\begin{tabular}{|c|c|c|c|}
\hline & CLR & TXMLO & \\
\hline & CLR & TXIHI & \\
\hline & CLR & TXILO & \\
\hline & LDAA & \#DB00 & ;SET 0 DB OF ATTENUATION \\
\hline & STAA & PORTA & \\
\hline & LDD & \#RADBUFF & ;SET A/D DATA BUFFER LOCATION IN BASE PAGE \\
\hline & STD & ADBFPTR & \\
\hline & LDX & \#MAXBUF 1 & ;SET FIRST MAX BUFFER LOCATION IN BASE PAGE \\
\hline & STX & MAXBUFF & \\
\hline & STX & TXBUFF & \\
\hline & LDAB & \#DMAXNUM & \\
\hline & ADDB & \#DMAXNUM & \\
\hline & STAB & MAXNUM & ;SET THE NUM MAX CHECK VALUE \\
\hline & $\mathrm{ABX}$ & & \\
\hline & STX & MAXFULL & ;SET BUFFER FULL TO 2xDMAXNUM \\
\hline & STX & TXBFULL & \\
\hline & LDD & \#DPERIOD & ;SET THE PERIOD SIZE \\
\hline & STD & PERIOD & \\
\hline & LDX & $\# \$ 0000$ & \\
\hline & STX & PERINDX & ;INITIALIZE PERIOD TIMER TO ZERO \\
\hline & LDAA & $\# \$ 00$ & \\
\hline & LDX & ADBFPTR & \\
\hline CLRAD: & STAA & $\$ 00, X$ & ;CLEAR THE RUNNING A/D BUFFER \\
\hline & INX & & \\
\hline & CPX & \#ADBFULL & \\
\hline & $\mathrm{BLO}$ & CLRAD & \\
\hline & LDX & \#MAXBUF1 & ;CLEAR THE FIRST MAXIMUM BUFFER \\
\hline CLMX1: & STAA & $\$ 00, X$ & \\
\hline & INX & & \\
\hline & CPX & \#MAX1FL & \\
\hline & BLS & CLMX1 & \\
\hline & LDX & \#MAXBUF2 & ;CLEAR THE SECOND MAXIMUM BUFFER \\
\hline CLMX2: & STAA & $\$ 00, \mathrm{X}$ & \\
\hline & INX & & \\
\hline & CPX & \#MAX2FL & \\
\hline & BLS & CLMX2 & \\
\hline & LDD & \#DWIND & ;SET DEFAULT INDEX WINDOW \\
\hline & STD & WINDOW & \\
\hline ; & & & \\
\hline * & 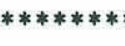 & 快 & $k * * * * * * * * * * * * * * * * * * * * * * * * * * * * * * * * * *$ \\
\hline & CLR & SCRTCH1 & ;USE AS SAIL MODE FLAG \& ADDR POINTER \\
\hline & LDAA & $\# \$ 7 \mathrm{E}$ & ;MUST WRITE JMP INSTS. \\
\hline & STAA & RJUMP & ;INTO SAIL WORK AREA \\
\hline & STAA & XJUMP & \\
\hline & STAA & JUMP2 & \\
\hline & LDD & \#SETUNAD & ;SET VECTORS TO INITIAL VALUES \\
\hline & STD & RVECT & \\
\hline & STD & XVECT & \\
\hline & CLI & & ;AND FINALLY ALLOW INTERRUPTS \\
\hline
\end{tabular}




\begin{tabular}{|c|c|c|c|}
\hline \multicolumn{4}{|c|}{; MAIN PROGRAM BODY } \\
\hline \multirow[t]{2}{*}{ LOOP: } & WAI & & ;WAIT TILL INTERRUPTED.. \\
\hline & BRA & LOOP & \\
\hline \multicolumn{4}{|c|}{$; * * * * * * * * * * * * * * * * * * * * * * * * * * * * * * * * * * * * * * * * * * * * * * * * * * * * * * * * * * * * * * * * * * * * * *$} \\
\hline \multicolumn{4}{|c|}{ 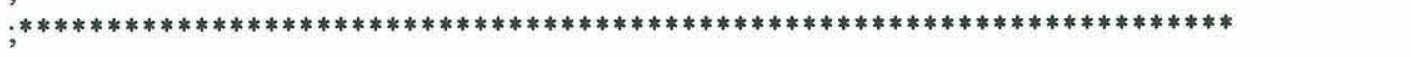 } \\
\hline \multicolumn{4}{|c|}{; } \\
\hline \multicolumn{4}{|c|}{; TIMER INTERUPT HANDLER } \\
\hline \multicolumn{4}{|c|}{ Fri, Jul 10, 1992, 13:02 } \\
\hline \multicolumn{4}{|c|}{$\quad$ Wed, Jul 22, 1992, 8:35 } \\
\hline \multicolumn{4}{|c|}{ Fri, Apr 23, 1993, 9:12 } \\
\hline \multicolumn{4}{|c|}{ Thu, May $20,1993,1: 52$ PM } \\
\hline \multirow{4}{*}{ OC2: } & & & \\
\hline & LDD & DTOC2 & ;GET LAST VALUE IN TOC2 \\
\hline & ADDD & \#SAMPLE & ;AND ADD ONE PERIOD TO IT \\
\hline & STD & DTOC2 & ;STORE BACK IN TOC2 \\
\hline \multirow[t]{5}{*}{, } & LDAA & $\# \$ F F$ & ;NORMALIZE THE A/D VALUE \\
\hline & SUBA & ADR1 & \\
\hline & CMPA & BIG & ;IS A/D VALUE GREATER THEN BIG? \\
\hline & BLO & TI1 & ;F IT IS STORE IN BIG ELSE MOVE ON \\
\hline & STAA & BIG & \\
\hline \multirow{7}{*}{; } & & & \\
\hline & LDX & ADBFPTR & ;GET THE CURRENT A/D POSITION \\
\hline & STAA & $\$ 00, \mathrm{X}$ & ;STORE THE VALUE \\
\hline & INX & & ;UPDATE THE POINTER \\
\hline & $\mathrm{CPX}$ & \#ADBFULL & ;CHECK IF AT LAST A/D STORAGE LOCATION \\
\hline & BLO & TI2 & ;IF NOT INC ADBPTR AND MOVE ON \\
\hline & LDX & \#RADBUFF & ;IF YES REINITIALIZE ADBPTR AND MOVE ON \\
\hline \multirow{3}{*}{$\begin{array}{l}\text { TI2: } \\
\text {; }\end{array}$} & STX & ADBFPTR & ;SAVE THE A/D STORAGE POINTER \\
\hline & LDX & \#RADBUFF & ;GET THE A/D BUFFER LOCATION \\
\hline & LDY & $\# \$ 0000$ & ;INITIALIZE THE SUMATION VALUE \\
\hline \multirow[t]{5}{*}{ SUMMING: } & LDAB & $\$ 00, \mathrm{X}$ & ;GET THE A/D VALUE TO ADD \\
\hline & $\mathrm{ABY}$ & & $; \mathrm{SUM}=\mathrm{SUM}+\mathrm{A} / \mathrm{D}$ \\
\hline & INX & & ;CONTINUE UNTIL ALL VALUES IN THE A/D \\
\hline & $\mathrm{CPX}$ & \#ADBFULL & ;BUFFER ARE SUMMED \\
\hline & BLO & SUMMING & \\
\hline ; & LDX & MAXBUFF & ;GET THE BUFFER TO WRITE TO \\
\hline & LDD & PERINDX & ;SETUP FOR TESTING MAX VALUES \\
\hline & STD & TEMPIND & \\
\hline \multirow{9}{*}{; } & CPD & WINDOW & ;SEE IF THE MAX IS CLOSE TO THE START OF \\
\hline & & & THE PERIOD \\
\hline & BHI & TI1_1_1 & ;IF NOT MOVE ON, OTHERWISE \\
\hline & CPY & $\$ 00, X^{-}$ & ;CHECK FOR A NEW MAXIMUM \\
\hline & BLO & TI1_2 & ;IF NOT GOTO CHECK FOR SECONDARY MAX'S \\
\hline & STY & $\$ 00, X$ & ;IF YES JUST REPLACE CURRENT MAX \\
\hline & LDD & TEMPIND & \\
\hline & STD & $\$ 10, \mathrm{X}$ & \\
\hline & BRA & TIENDJ & \\
\hline
\end{tabular}




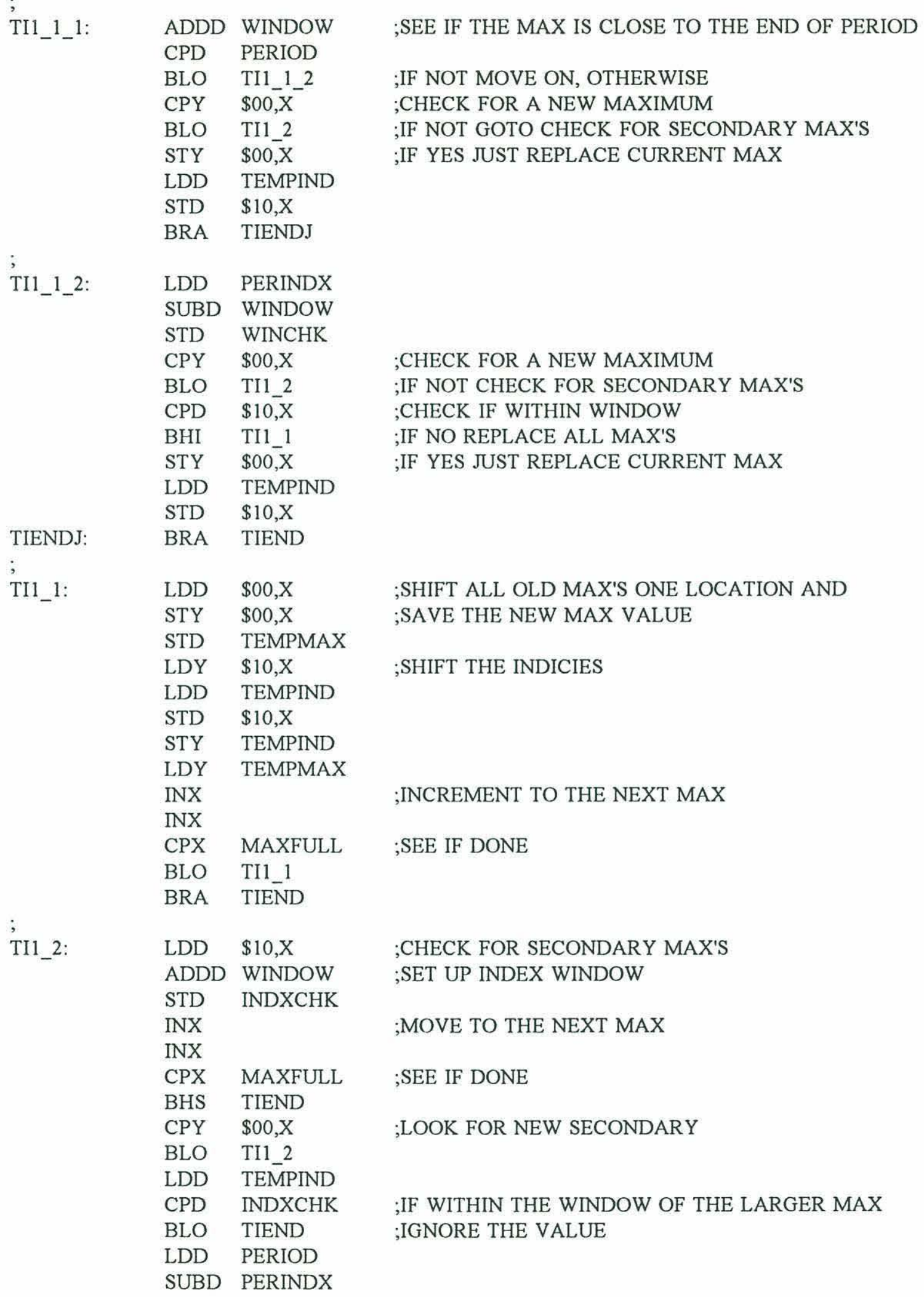




\begin{tabular}{|c|c|c|c|}
\hline & CPD & WINDOW & -IF ON THF SCRFFN WRAP IN WINDOW IGNORE \\
\hline \multicolumn{4}{|l|}{, } \\
\hline & LDD & PERINDX & \\
\hline & SUBD & WINDOW & \\
\hline & CPD & $\$ 10, \mathrm{X}$ & ;IF WITHIN THE WINDOW OF THE SECONDARY \\
\hline & BHI & TI1_3 & \\
\hline & STY & $\$ 00, \mathrm{X}$ & ;JUST REPLACE THE ONE VALUE \\
\hline & LDD & PERINDX & \\
\hline & STD & $\$ 10, \mathrm{X}$ & \\
\hline & BRA & TIEND & \\
\hline \multicolumn{4}{|c|}{ (a) } \\
\hline \multirow[t]{12}{*}{ TI1_3: } & LDD & $\$ 00, \mathrm{X}$ & ;ELSE REPLACE AND SHIFT OLD VALUES DOWN \\
\hline & STY & $\$ 00, \mathrm{X}$ & \\
\hline & STD & TEMPMAX & \\
\hline & LDY & $\$ 10, \mathrm{X}$ & \\
\hline & LDD & TEMPIND & \\
\hline & STD & $\$ 10, \mathrm{X}$ & \\
\hline & STY & TEMPIND & \\
\hline & LDY & TEMPMAX & \\
\hline & INX & & \\
\hline & INX & & \\
\hline & CPX & MAXFULL & \\
\hline & BLO & TI1_3 & \\
\hline \multirow{5}{*}{ TIEND: } & & & \\
\hline & LDX & PERINDX & ;GET THE PERIOD COUNTER AND INCREMENT \\
\hline & INX & & \\
\hline & CPX & PERIOD & ;IS THE PERIOD COMPLETED? \\
\hline & BLO & TI11 & \\
\hline \multicolumn{4}{|l|}{; } \\
\hline & INC & MAXFLG & \\
\hline & \multicolumn{2}{|c|}{;ARE WE IN MAXIMUMS BUFFER 1 OR 2? } & ;ARE WE IN MAXIMUMS BUFFER 1 OR 2? \\
\hline & CLR & TX1FLG & ;CLEAR BUFFER 1 TX'D FLAG \\
\hline & LDY & \#MAXBUF2 & \\
\hline & BRA & TI8 & \\
\hline \multirow[t]{2}{*}{ TI7: } & CLR & TX2FLG & ;CLEAR BUFFER 2 TX'D FLAG \\
\hline & LDY & \#MAXBUF1 & \\
\hline \multirow[t]{4}{*}{ TI8: } & STY & MAXBUFF & ;UPDATE THE BUFFER TO USE \\
\hline & LDAB & MAXNUM & \\
\hline & $\mathrm{ABY}$ & & \\
\hline & STY & MAXFULL & ;UPDATE THE FULL BUFFER VALUE \\
\hline \multirow{6}{*}{, } & BRSET & AGCFLG,\$01,TI1C & ;IF MANUAL GAIN SET DON'T CHECK AGC \\
\hline & LDAA & BIG & ;TEST IF GAIN NEEDS TO BE DECREASED \\
\hline & CMPA & \#ATTNINC & ;IF NOT MOVE ON \\
\hline & BLO & TI9 & \\
\hline & JSR & ATTN & ;GO TO ATTN FOR MORE ATTENUATION \\
\hline & BRA & TI10 & \\
\hline \multirow[t]{3}{*}{ TI9: } & CMPA & \#ATTNDEC & ;TEST IF GAIN NEEDS TO BE INCREASED \\
\hline & BHI & TI10 & ;IF NOT MOVE ON \\
\hline & JSR & INC & ;GO TO INC FOR LESS ATTENUATION \\
\hline
\end{tabular}




\begin{tabular}{|c|c|c|c|}
\hline TI10: & CLR & BIG & ;RESET BIG FOR NEXT PERIOD \\
\hline , & LDX & $\# \$ 0000$ & ;CLEAR THE PERIOD COUNTER \\
\hline TI11: & STX & PERINDX & \\
\hline & LDAA & $\# \$ 40$ & ;RESET TIMER FLAGS \\
\hline & STAA & TFLG1 & \\
\hline & RTI & & \\
\hline 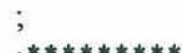 & & & \\
\hline$; * * * * * * * *$ & $* * * * * *$ & $* * * * * * * * * * * *$ & $k * * * * * * * * * * * * * * * * * * * * * * * *$ \\
\hline ; SCI INTH & UPT HAI & NDLER (SAIL I & RIVER) \\
\hline Sat & 12,195 & $90,20: 34 \mathrm{COM}$ & ACT BY 20 BYTES BY USING RSET: \\
\hline Su & ay 13,19 & $90,12: 36 \mathrm{COM}$ & ACT BY USING CONDITIONAL ASSEMBLY \\
\hline Sur & ay 13,19 & $90,12: 37$ ANI & BY USING TTY FOR PROMPT \\
\hline Mc & ep 17,19 & $90,19: 20 \mathrm{ADD}$ & N4 TO TTY \\
\hline SCI: & BRCLR & SCSR,\$OE,CK & HAR ;TEST FOR VALID CHAR \\
\hline ;\& & LDAA & SCSR & ;TEST OR.NF.FE \\
\hline ; & ANDA & $\# \$ O E$ & \\
\hline ; & BEQ & CKCHAR & ;IF GOOD, GO ON.. \\
\hline & LDAA & SCDR & ;READ BAD CHAR TO CLEAR? \\
\hline ECHO: & NOP & & ; RECEIVE AN ECHO CHARACTER (\& IGNORE!) \\
\hline SCIXIT: & RTI & & ; \& EXIT \\
\hline ; & & & \\
\hline CKCHAR: & BRSET & SCSR,\$20,RE & \\
\hline ;\& & LDAA & SCSR & ;TEST DA \\
\hline ; & ANDA & $\# \$ 20$ & \\
\hline ; & BNE & READD & \\
\hline & JMP & CHECKX & ;IF NOT, XMIT \\
\hline ; & & & \\
\hline READD: & LDAA & SCDR & ;READ DATA TO A.. \\
\hline & $\mathrm{TAB}$ & & ;AND COPY TO B \\
\hline ; & LDX & \#PARITY & ;POINT PARITY TABLE \\
\hline ; & $\mathrm{ABX}$ & & ;POINT INTO PARITY TABLE \\
\hline ; & LDAB & $0, \mathrm{X}$ & ;GET PARITY WORD \\
\hline ; & BNE & SCIXIT & ;REJECT IF NOT EVEN PARITY \\
\hline ; & ANDA & $\# \$ 7 F$ & ;ELSE REMOVE PARITY FOR SUBSEQUENT TESTS \\
\hline & TAB & & ;(ALSO COPY TO B) \\
\hline & CMPA & \#'\#' & ;IS IT '\#'? \\
\hline & BEQ & GOTNUM & \\
\hline & JMP & RJUMP & ;ELSE GO THRU VECTOR \\
\hline ATYIT. & & & \\
\hline ATXIT: & JMP & SCIXIT & ;NO FURTHER USE FOR THIS CHAR, EXIT \\
\hline ; SAIL ADI & SS RECO & GNIZE SECTI & \\
\hline GOTNUM: & LDAB & $\# 01$ & ; GOT \# CODE \\
\hline & STAB & SCRTCH1 & ;SET SAIL FLAG TO 01 \\
\hline & BCLR & SCCR2,\$D2 & ;TURN OFF XMIT INTERRUPTS \\
\hline ;\& & LDAA & SCCR2 & \\
\hline , & ANDA & $\# \$ 2 \mathrm{C}$ & ;TURN OFF XMIT INTERRUPTS \\
\hline , & STAA & SCCR2 & \\
\hline & LDD & \#READAD & ;POINT READ ADDRESS \\
\hline
\end{tabular}




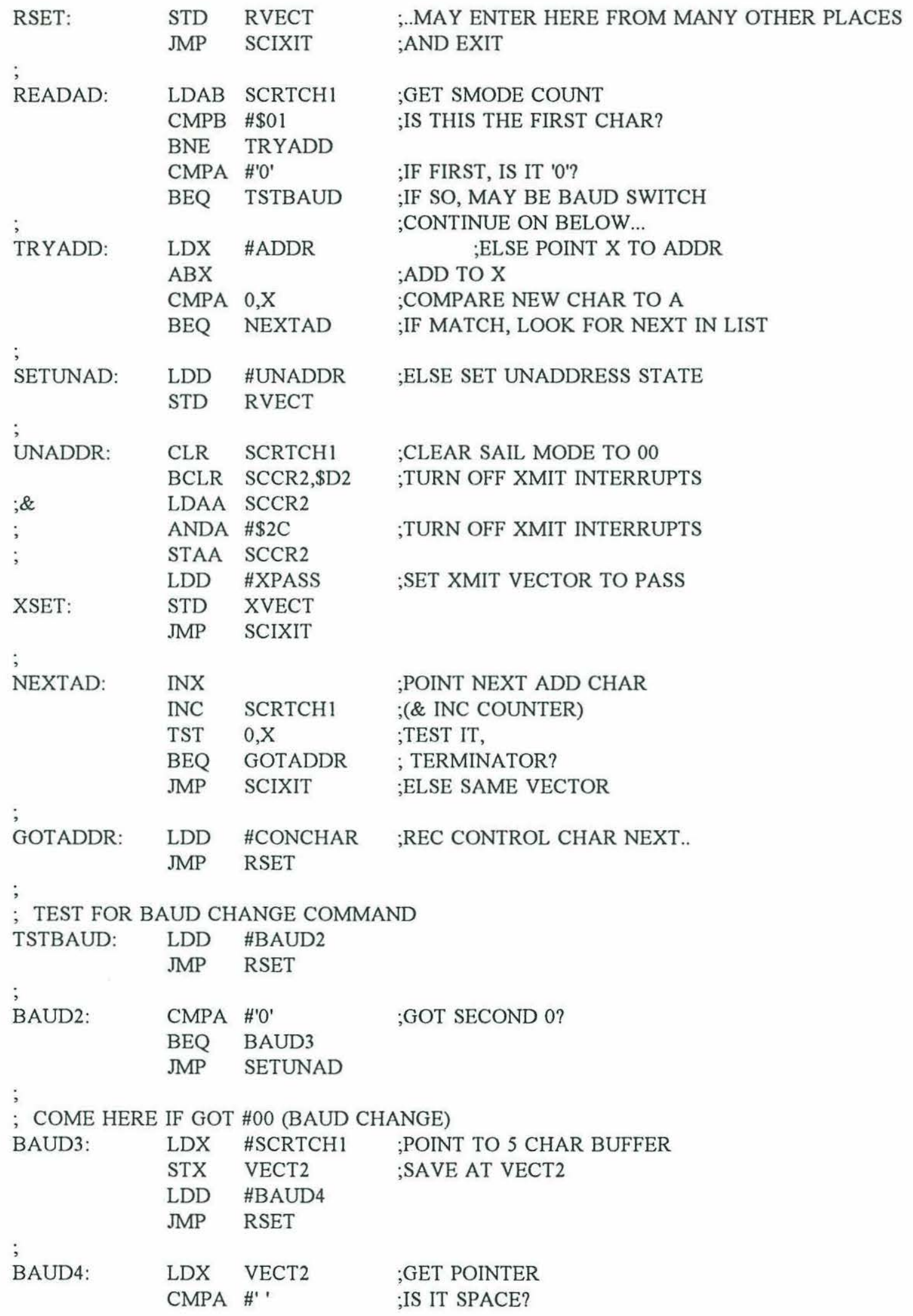




\begin{tabular}{|c|c|c|c|}
\hline & BNE & BAUD5 & \\
\hline & LDAA & $\#^{\prime} 0^{\prime}$ & ;..CHANGE SPACES TO 0'S \\
\hline BAUD5: & STAA & $0, \mathrm{X}$ & ;STORE CHAR \\
\hline & INX & & ;ADVANCE POINTER \\
\hline & CPX & \#SCRTCH1+5 & ;PAST END OF 5 CHAR BUFFER? \\
\hline & BEQ & BAUD6 & \\
\hline & STX & VECT2 & ;IF NOT DONE, SAVE POINTER \\
\hline & JMP & SCIXIT & ;AND GET MORE \\
\hline BAUD6: & LDX & SCRTCH $1+3$ & ;GET LAST TWO CHARS \\
\hline & $\mathrm{CPX}$ & $\# \$ 3030$ & ;ARE LAST TWO CHARS BOTH $0 ?$ \\
\hline & BNE & WAITBRK & ;IF NOT VALID, SET UP TO WAIT FOR BREAK \\
\hline ; IF LAST TV & CHARS & ARE 00, CHEC & K BUFFER AGAINST DATA TABLES FOR A MATCH \\
\hline & LDD & \#BAUDTBL & ;SET UP TABLE POINTER \\
\hline & STD & VECT2 & ;AND STORE IN JUMP2 VECTOR \\
\hline ; TRY EACH & ABLE S & EPARATELY, 0 & AS 1ST CHAR SIGNALS END OF TABLE \\
\hline BTRY: & LDX & VECT2 & ;GET TABLE POINTER \\
\hline & LDAA & $0, \mathrm{X}$ & ; AND TABLE ELEMENT \\
\hline & BEQ & WAITBRK & ; IF $=00$, WAS NO MATCH, WAIT FOR BREAK \\
\hline ; COMPARE & UFFER & WITH ONE TAB & LE ENTRY \\
\hline & LDX & \#SCRTCH1 & ;INIT BUFFER POINTER TO TOP \\
\hline & STX & XVECT & ; \& SAVE IN XMIT JUMP VECTOR \\
\hline ; COMPARE & $\mathrm{ACH} \mathrm{CH}$ & IARACTER & \\
\hline COMPARE: & LDX & XVECT & ;GET BUFFER POINTER \\
\hline & LDAA & $0, \mathrm{X}$ & ; TO GET BUFFER CHAR TO A \\
\hline & INX & & ; INX BUF POINT WHILE WE'RE HERE \\
\hline & STX & XVECT & ; \& SAVE.. \\
\hline & LDX & VECT2 & ;NOW GET TABLE POINTER \\
\hline & CMPA & $0, \mathrm{X}$ & ; \& USE TO COMPARE CHARS \\
\hline & BNE & NOMATCH & \\
\hline CMATCH: & INX & & ;IF MATCH, MOVE AHEAD TABLE POINTER \\
\hline & LDAA & $0, \mathrm{X}$ & ;LOOK AT NEXT TABLE ELEMENT \\
\hline & BEQ & MATCH & ; IF IT'S 00, FULL MATCH! \\
\hline & STX & VECT2 & ; ELSE SAVE TABLE POINTER \\
\hline & BRA & COMPARE & ; AND TRY NEXT CHAR \\
\hline NOMATCH: & INX & & ;INC TABLE POINTER \\
\hline & LDAA & $0, \mathrm{X}$ & ;AND LOOK FOR 00 \\
\hline & BNE & NOMATCH & ;LOOP TILL IT'S FOUND \\
\hline & INX & & ;INC OVER NULL \\
\hline & INX & & ; \& BAUD SET BYTE \\
\hline & STX & VECT2 & ;AND REPLACE TO HOLDING LOCATION \\
\hline & BRA & BTRY & ;GO BACK TO CHECK NEXT TABLE \\
\hline 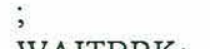 & & & \\
\hline WAITBRK: & LDAA & $\# \$ 0 \mathrm{C}$ & ;TURN OFF SCI INTERRUPTS \\
\hline & STAA & SCCR2 & ; (REQUIRES TOF INTERRUPT TO RESTART) \\
\hline & JMP & SETUNAD & ;AND SET VECTORS FOR NEW ADDRESS.. \\
\hline
\end{tabular}




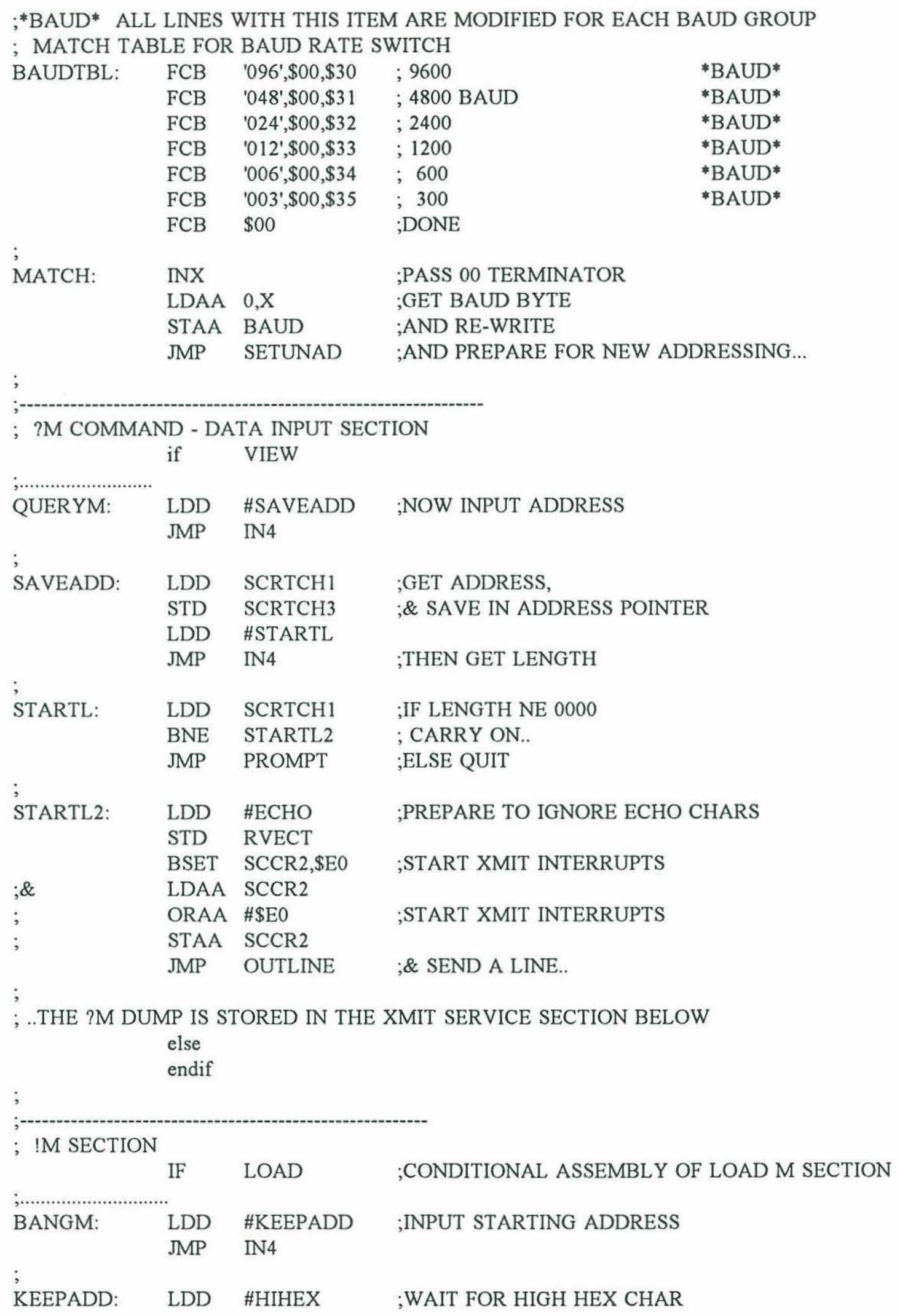




\begin{tabular}{|c|c|c|c|}
\hline & JMP & RSET & \\
\hline \multirow{9}{*}{ HIHEX: } & JSR & ASCHEX & :ATTEMPT CONVERT TO HEX \\
\hline & $\mathrm{BCS}$ & TERMIN & ;CHECK TERMINATOR IF NOT.. \\
\hline & ASLA & & \\
\hline & ASLA & & ;MOVE OVER NIBBLE \\
\hline & ASLA & & \\
\hline & ASLA & & \\
\hline & STAA & SCRTCH5 & \\
\hline & LDD & \#LOWHEX & \\
\hline & JMP & RSET & \\
\hline \multirow{3}{*}{ LOWHEX: } & JSR & ASCHEX & :CONVERT LOW \\
\hline & $\mathrm{BCC}$ & STASH & \\
\hline & JMP & PROMPT & ;PROMPT IF BAD SECOND CHAR \\
\hline \multirow[t]{7}{*}{ STASH: } & LDX & SCRTCH1 & ;GET POINTER \\
\hline & ORAA & SCRTCH5 & ;COMBINE NIBBLES \\
\hline & STAA & $0, \mathrm{X}$ & ;STORE IT.. \\
\hline & INX & & ;INC \& \\
\hline & STX & SCRTCH1 & ;RESTORE POINTER \\
\hline & LDD & \#HIHEX & \\
\hline & JMP & RSET & \\
\hline \multirow{5}{*}{; TERMIN: } & & & \\
\hline & ANDA & $\# \$ 7 F$ & ;MASK OFF FLAG BIT \\
\hline & CMPA & $\# \$ 0 D$ & ;IS IT CR? \\
\hline & BNE & TERMIN2 & \\
\hline & JMP & PROMPT & \\
\hline \multirow{5}{*}{; TERMIN2: } & & & \\
\hline & CMPA & \#' ' & ;IS IT SPACE? \\
\hline & BNE & TERMIN3 & \\
\hline & LDD & \#HIHEX & \\
\hline & JMP & RSET & \\
\hline \multirow{5}{*}{ TERMIN3: } & & & \\
\hline & CMPA & \#';' & ;OR ';' ? \\
\hline & BNE & TERMIN4 & \\
\hline & LDD & \#BANGM & \\
\hline & BRA & PASSLF & \\
\hline 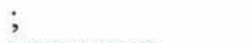 & & & \\
\hline \multirow{3}{*}{ TERMIN4: } & CMPA & \#',' & ;OR ','? \\
\hline & BNE & TERMIN5 & \\
\hline & LDD & \#SETHIHX & \\
\hline \multirow[t]{3}{*}{ PASSLF: } & STD & VECT2 & \\
\hline & LDD & \#PASSLF2 & \\
\hline & JMP & RSET & \\
\hline \multirow{2}{*}{\multicolumn{2}{|c|}{; }} & & \\
\hline & & $\# \$ 0 \mathrm{~A}$ & ;GOT LF YET? \\
\hline & BNE & PXIT & \\
\hline & JMP & ЛUP2 & ;IF YES, EXIT THRU JMP2 \\
\hline PXIT: & JMP & SCIXIT & ;ELSE WAIT FOR MORE CHARS.. \\
\hline SETHIHX: & LDD & \#HIHEX & ;IF ',' THEN PREP FOR MORE HEX \\
\hline
\end{tabular}




$\begin{array}{llll} & \text { JMP } & \text { RSET } & \\ \text { TERMIN5: } & \begin{array}{l}\text { JMP } \\ \text { endif }\end{array} & \text { PROMPT } & \text {;TO PROMPT IF NOT VALID CHAR }\end{array}$

\begin{tabular}{|c|c|c|c|}
\hline \multirow{2}{*}{\multicolumn{4}{|c|}{; IN4 HEX DIGITS }} \\
\hline \multirow{2}{*}{\multicolumn{4}{|c|}{$\begin{array}{l}\text { IN4 HEX DIGITS } \\
\text { INPUTS HEX DIGITS TILL TERMINATED BY A SPACE OR }\end{array}$}} \\
\hline & & & \\
\hline \multicolumn{4}{|c|}{ BY A CR. (OR BY UNADDRESS). RESULT LEFT AT SCRTCH 1,2} \\
\hline \multicolumn{4}{|c|}{ EXITS THRU JUMP2 WHEN DONE (TARGET IN D ON ENTRY) } \\
\hline \multirow[t]{5}{*}{ IN4: } & STD & VECT2 & ;STORE EXIT VECTOR \\
\hline & CLR & SCRTCH1 & ;CLEAR INPUT BUFFER \\
\hline & CLR & SCRTCH2 & \\
\hline & LDD & \#IN4HEX & ;POINT CHAR RECEIVER \\
\hline & JMP & RSET & \\
\hline \multirow{15}{*}{ IN4HEX: } & JSR & ASCHEX & :CONVERT TO HEX \\
\hline & $\mathrm{BCS}$ & CKEND & ;F NOT HEX, CHECK FOR END \\
\hline & PSHA & & ;SAVE.. \\
\hline & LDAA & SCRTCH1 & ;HI TO A \\
\hline & LDAB & SCRTCH2 & \\
\hline & ASLD & & ;SHIFT OVER FOUR \\
\hline & ASLD & & \\
\hline & ASLD & & \\
\hline & ASLD & & \\
\hline & STAA & SCRTCH1 & ;RETURN HIGH \\
\hline & STAB & SCRTCH2 & ;AND B \\
\hline & PULA & & ;RETRIEVE NEW NIBBLE \\
\hline & ORAA & SCRTCH2 & ;ADD NEW NIBBLE \\
\hline & STAA & SCRTCH2 & ; \& UPDATE \\
\hline & JMP & SCIXIT & \\
\hline \multirow{6}{*}{ CKEND: } & ANDA & $\# \$ 7 F$ & ;CLEAN OFF MS FLAG BIT \\
\hline & CMPA & \#"' & ;IS IT SPACE? \\
\hline & BEQ & INEXIT & \\
\hline & CMPA & $\# \$ 0 \mathrm{D}$ & ;IS IT CR? \\
\hline & BEQ & INEXIT & \\
\hline & JMP & SCIXIT & ;ELSE, GET ANOTHER CHAR \\
\hline \multirow{3}{*}{ INEXIT: } & JMP & JUMP2 & ;IF DONE, CARRY ON THRU VECTOR. \\
\hline & & & \\
\hline & & & \\
\hline \multicolumn{4}{|c|}{; INPUT ONE HEX BYTE AND CONTINUE THRU JUMP2 } \\
\hline \multirow[t]{7}{*}{ INBYTE: } & ASLA & & ;MOVE OVER \\
\hline & ASLA & & \\
\hline & ASLA & & \\
\hline & ASLA & & \\
\hline & STAA & SCRTCH5 & ;SAVE \\
\hline & LDD & \#INBYTE2 & ;POINT TO NEXT SEGMENT \\
\hline & JMP & RSET & \\
\hline
\end{tabular}




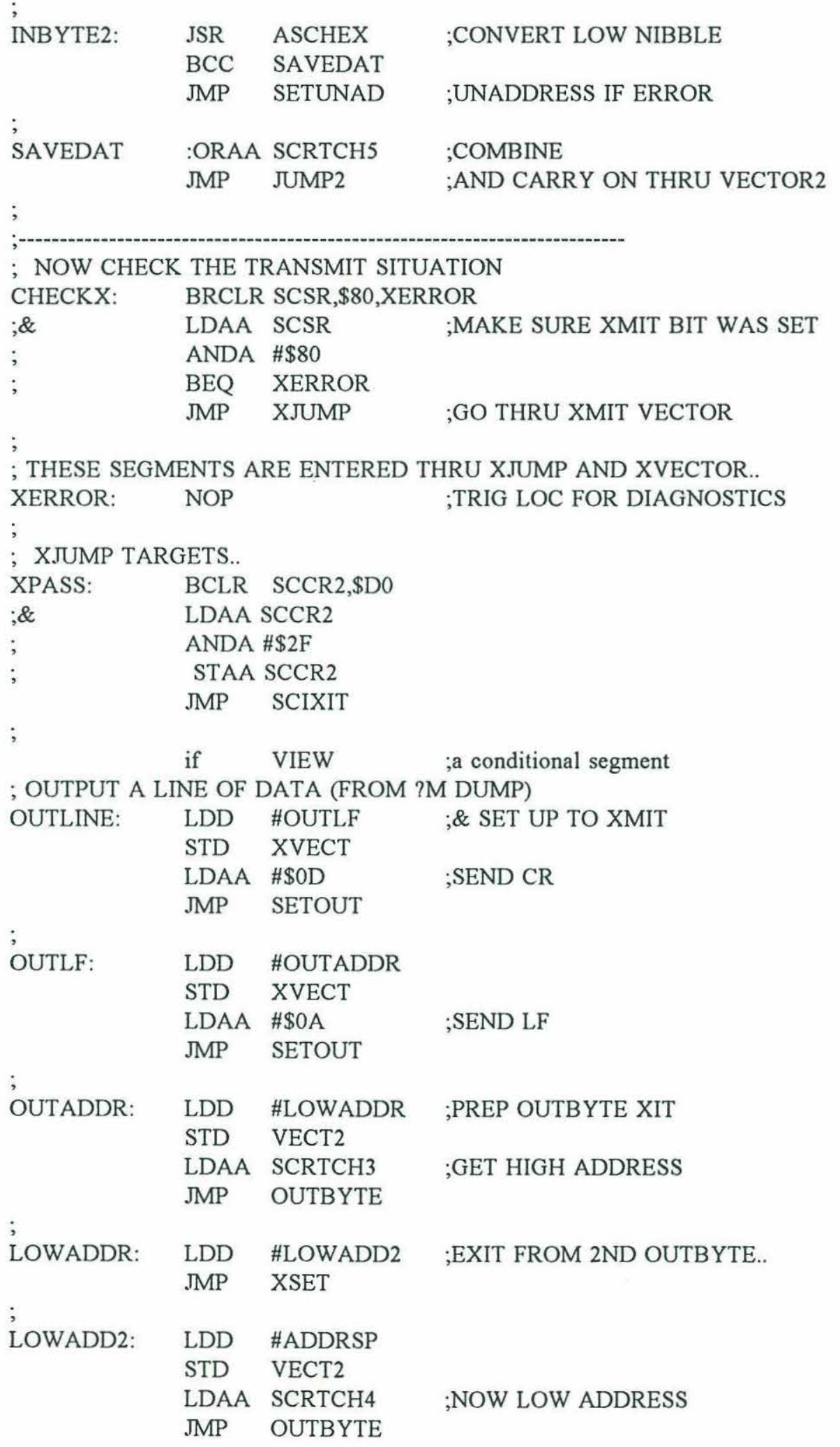




\begin{tabular}{|c|c|c|c|}
\hline ADDRSP: & $\begin{array}{l}\text { LDD } \\
\text { JMP }\end{array}$ & $\begin{array}{l}\text { \#SENDSP } \\
\text { XSET }\end{array}$ & ;EXIT FROM 2ND OUTBYTE... \\
\hline ; & & & \\
\hline \multirow[t]{4}{*}{ SENDSP: } & LDD & \#OUTDATA & \\
\hline & STD & XVECT & \\
\hline & LDAA & $\# \$ 20$ & ;SEND SPACE \\
\hline & JMP & SETOUT & \\
\hline \multicolumn{4}{|c|}{ 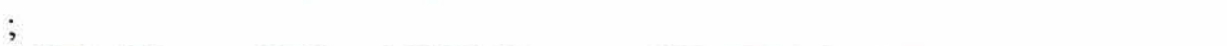 } \\
\hline \multirow[t]{7}{*}{ OUTDATA: } & LDX & SCRTCH3 & ;GET ADDR POINTER \\
\hline & LDAA & $0, \mathrm{X}$ & ;TO GET DATA BYTE \\
\hline & PSHA & & ;SAVE \\
\hline & LDD & \#DECLEN & \\
\hline & STD & VECT2 & ;PREP XIT VECTOR \\
\hline & PULA & & ;RETRIEVE DATA BYTE \\
\hline & JMP & OUTBYTE & ;\& SEND IT \\
\hline \multirow{3}{*}{ DECLEN: } & & & \\
\hline & LDD & \#DECLEN2 & ;EXIT FROM OUTBYTE2.. \\
\hline & JMP & XSET & \\
\hline \multirow[t]{5}{*}{ DECLEN2: } & LDX & SCRTCH1 & ;GET LENGTH \\
\hline & DEX & & ;DECREMENT \\
\hline & STX & SCRTCH1 & ;\& RETURN \\
\hline & BNE & SAYMORE & ; MORE DATA? \\
\hline & JMP & PROMPT & ;PROMPT IF DONE \\
\hline \multirow{9}{*}{ SAYMORE: } & & & \\
\hline & LDX & SCRTCH3 & ;GET ADDR \\
\hline & INX & & ;INCREMENT \\
\hline & STX & SCRTCH3 & ;REPLACE \\
\hline & XGDX & & ;X REG TO ACCD \\
\hline & LSRD & & ;LSB TO C \\
\hline & $\mathrm{BCS}$ & OUTDATA & ;NEXT BYTE NOW IF ODD \\
\hline & ANDB & $\# \$ 07$ & ;CHECK FOR XXX0 (LINE END) \\
\hline & BNE & SENDSP & ;IF ONLY EVEN, SEND SP \\
\hline \multirow{5}{*}{; } & LDAA & \#';' & ;IF NEW LINE, SEND CONTINUATION \\
\hline & JSR & GENPAR & \\
\hline & STAA & SCDR & \\
\hline & LDD & \#OUTLINE & ;\& PREP FOR NEW LINE.. \\
\hline & $\begin{array}{l}\text { JMP } \\
\text { endif }\end{array}$ & XSET & \\
\hline \multicolumn{4}{|l|}{; } \\
\hline \multirow{9}{*}{$\begin{array}{l}\text {; OUTPUTS } \\
\text { OUTBYTE: }\end{array}$} & BYTE IN & J A AS TWO & X CHARS, EXITS VIA VECT $2 .$. \\
\hline & STAA & SCRTCH5 & ;STASH FOR LATER \\
\hline & LSRA & & \\
\hline & LSRA & & ;MOVE OVER \\
\hline & LSRA & & \\
\hline & LSRA & & \\
\hline & JSR & HEXASC & ;CONVERT \\
\hline & PSHA & & ;AND SAVE.. \\
\hline & LDD & \#OUTBYT2 & \\
\hline
\end{tabular}




\begin{tabular}{|c|c|c|c|}
\hline & $\begin{array}{l}\text { STD } \\
\text { PULA } \\
\text { JMP }\end{array}$ & $\begin{array}{l}\text { XVECT } \\
\text { SETOUT }\end{array}$ & ;RECOVER CHARACTER \\
\hline & & & \\
\hline \multirow{4}{*}{ OUTBYT2: } & LDAA & SCRTCH5 & \\
\hline & ANDA & $\# \$ O F$ & ;CLEAN UP \\
\hline & JSR & HEXASC & \\
\hline & JMP & OUTJMP & ;CONVERT, SEND \& JUMP THRU VECT2 \\
\hline \\
\hline \multicolumn{4}{|c|}{; SEND A PROMPT FROM THE MONITOR, THEN WAIT FOR CHARS } \\
\hline \multirow[t]{2}{*}{ PROMPT: } & LDX & \#PLIST & \\
\hline & JMP & TTYO & \\
\hline \multirow[t]{2}{*}{ PLIST: } & FCB & \$OD,\$OA, ':',\$EE,\$ & $\$ 90$, SCRTCH1,\$AA \\
\hline & FDB & PJUMP & \\
\hline \multirow[t]{2}{*}{ PJUMP: } & LDAA & SCRTCH1 & \\
\hline & JMP & CONCHAR & \\
\hline \multicolumn{4}{|c|}{; INITIATE AN OUTPUT SEQUENCE } \\
\hline \multirow[t]{3}{*}{ SETOUT: } & JSR & GENPAR & \\
\hline & STAA & SCDR & ;SEND FIRST CHAR \\
\hline & BSET & SCCR2,\$C0 & \\
\hline ;\& & LDAA & SCCR2 & \\
\hline ; & ORAA & $\# \$ C 0$ & ;TURN ON INTERRUPTS IF REQ'D \\
\hline \multirow[t]{3}{*}{; } & STAA & SCCR2 & \\
\hline & LDD & \#ЕCHO & ;\& PREPARE TO ECHO \\
\hline & JMP & RSET & \\
\hline \multicolumn{4}{|c|}{; SENDS ETX \& CARRIES ON THRU VECT2 } \\
\hline XETX: & LDAA & $\# \$ 03$ & ;SEND ETX \\
\hline \multirow[t]{3}{*}{ OUTJMP: } & JSR & GENPAR & ;MAY ENTER HERE... \\
\hline & STAA & SCDR & ;DRAGON AGAIN? \\
\hline & JMP & JUMP2 & ;NOW THRU JUMP2 \\
\hline \multicolumn{4}{|r|}{ 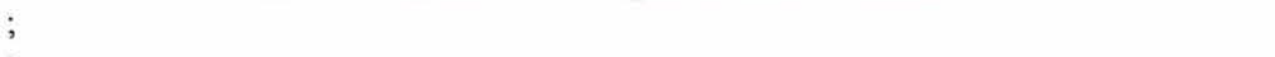 } \\
\hline \\
\hline \multicolumn{4}{|c|}{ 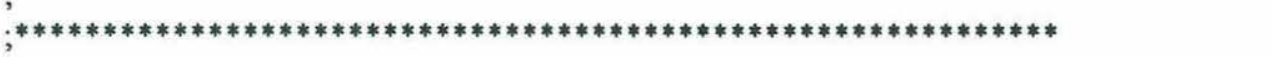 } \\
\hline \multicolumn{4}{|c|}{; SUBROUTINES USED BY PTR DRIVER...... } \\
\hline \multirow{6}{*}{ ATTN: } & LDAA & PORTA & GET THE VALUE IN PORT A \\
\hline & CMPA & \#DB00 & ;IS O DB SET? \\
\hline & $\mathrm{BNE}$ & ATTN1 & ;IF NO GO ON \\
\hline & LDAA & \#DB03 & ;IF YES SET 3 DB \\
\hline & STAA & PORTA & \\
\hline & BRA & ATTNF & ;EXIT \\
\hline \multirow[t]{5}{*}{ ATTN1: } & CMPA & \#DB03 & ;IS 3 DB SET? \\
\hline & $\mathrm{BNE}$ & ATTN2 & ;IF NO GO ON \\
\hline & LDAA & \#DB09 & ;IF YES SET 9 DB \\
\hline & STAA & PORTA & \\
\hline & BRA & ATTNF & ;EXIT \\
\hline \multirow[t]{2}{*}{ ATTN2: } & CMPA & \#DB12 & ;IS 12 DB SET? \\
\hline & BNE & ATTN3 & ;IF NO GO ON \\
\hline
\end{tabular}




\begin{tabular}{|c|c|c|c|}
\hline & LDAA & \#DB18 & ;IF YES SET 18 DB \\
\hline & STAA & PORTA & \\
\hline & BRA & ATTNF & ;EXIT \\
\hline \multirow[t]{5}{*}{ ATTN3: } & CMPA & \#DB18 & ;IS 18 DB SET? \\
\hline & BNE & ATTN4 & ;F NO GO ON \\
\hline & LDAA & \#DB24 & ;IF YES SET 24 DB \\
\hline & STAA & PORTA & \\
\hline & BRA & ATTNF & ;EXIT \\
\hline \multirow[t]{5}{*}{ ATTN4: } & CMPA & \#DB24 & ;IS 24 DB SET? \\
\hline & BNE & ATTN5 & ;IF NO GO ON \\
\hline & LDAA & \#DB30 & ;IF YES SET $30 \mathrm{DB}$ \\
\hline & STAA & PORTA & \\
\hline & BRA & ATTNF & ;EXIT \\
\hline \multirow[t]{3}{*}{ ATTN5: } & CMPA & \#DB30 & ;IS 30 DB SET? \\
\hline & BNE & ATTN6 & ;IF NO GO ON \\
\hline & BRA & ATTNF & ;IF YES CAN'T CHANGE ANYMORE \\
\hline \multirow{2}{*}{ ATTN6: } & LDAA & \#DB00 & ;IF NON OF THE ABOVE ARE SET, SET 0 DB \\
\hline & STAA & PORTA & \\
\hline ATTNF & RTS & & . \\
\hline \multirow[t]{6}{*}{ INC: } & LDAA & PORTA & ;GET THE VALUE IN PORT A \\
\hline & CMPA & \#DB30 & ;IS 30 DB SET? \\
\hline & BNE & INC1 & ;IF NO GO ON \\
\hline & LDAA & \#DB24 & ;IF YES SET 24 DB \\
\hline & STAA & PORTA & \\
\hline & BRA & INCF & ;EXIT \\
\hline \multirow[t]{5}{*}{ INC1: } & CMPA & \#DB24 & ;IS 24 DB SET? \\
\hline & BNE & INC2 & ;IF NO GO ON \\
\hline & LDAA & \#DB18 & ;IF YES SET 18 DB \\
\hline & STAA & PORTA & \\
\hline & BRA & INCF & ;EXIT \\
\hline \multirow{5}{*}{ INC2: } & CMPA & \#DB18 & ;IS 18 DB SET? \\
\hline & BNE & INC3 & ;IF NO GO ON \\
\hline & LDAA & \#DB12 & ;IF YES SET 12 DB \\
\hline & STAA & PORTA & \\
\hline & BRA & INCF & ;EXIT \\
\hline \multirow[t]{5}{*}{ INC3: } & CMPA & \#DB12 & ;IS 12 DB SET? \\
\hline & BNE & INC4 & ;IF NO GO ON \\
\hline & LDAA & \#DB09 & ;IF YES SET 9 DB \\
\hline & STAA & PORTA & \\
\hline & BRA & INCF & \\
\hline \multirow[t]{5}{*}{ INC4: } & CMPA & \#DB09 & ;IS 9 DB SET? \\
\hline & BNE & INC5 & ;IF NO GO ON \\
\hline & LDAA & \#DB03 & ;IF YES SET 3 DB \\
\hline & STAA & PORTA & \\
\hline & BRA & INCF & \\
\hline \multirow[t]{2}{*}{ INC5: } & LDAA & \#DB00 & ;IF NON OF THE ABOVE ARE SET, SET O DB \\
\hline & STAA & PORTA & \\
\hline INCF & RTS & & \\
\hline
\end{tabular}




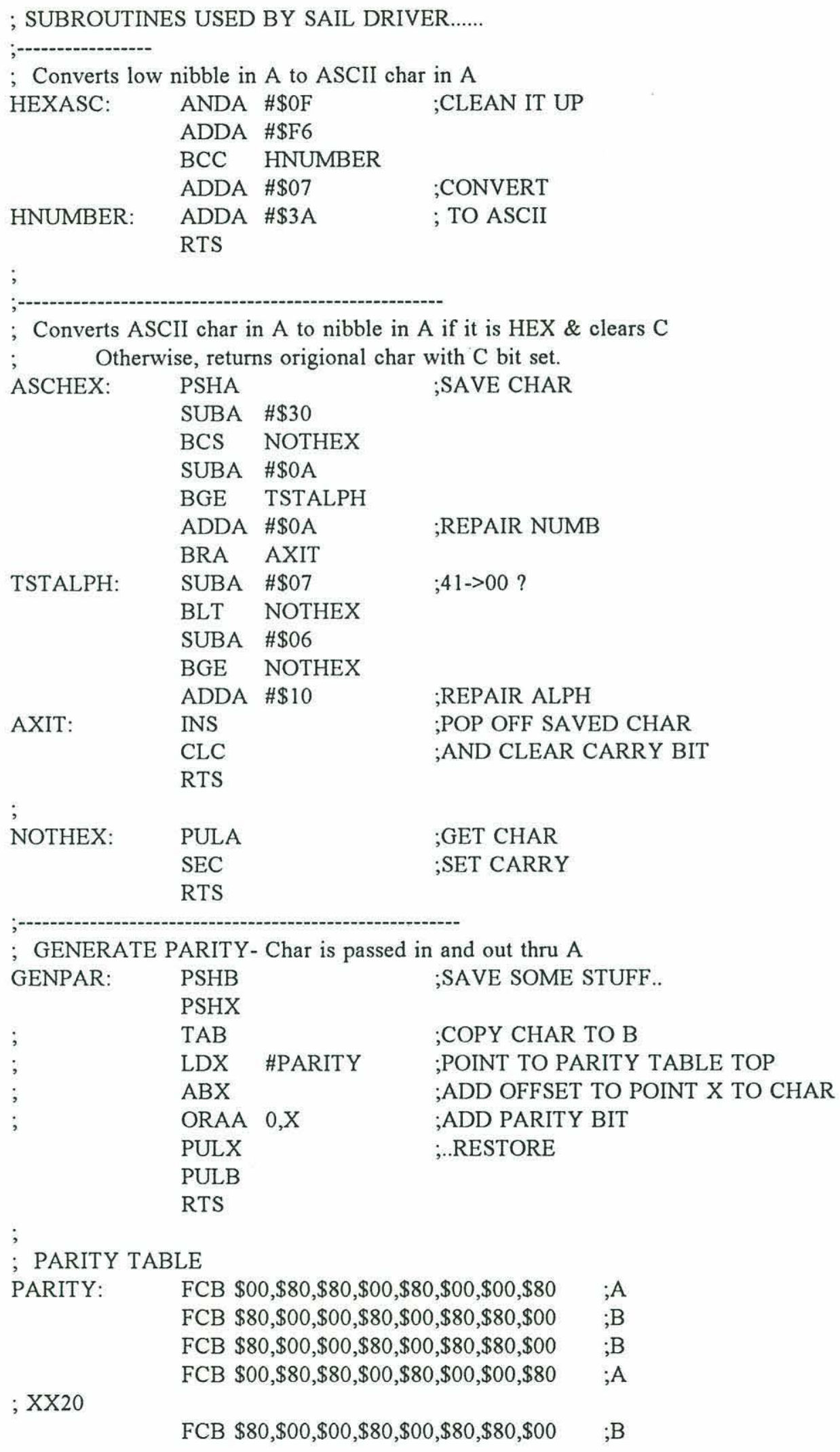


FCB $\$ 00, \$ 80, \$ 80, \$ 00, \$ 80, \$ 00, \$ 00, \$ 80 \quad ; A$

FCB $\$ 00, \$ 80, \$ 80, \$ 00, \$ 80, \$ 00, \$ 00, \$ 80 \quad ; A$

FCB $\$ 80, \$ 00, \$ 00, \$ 80, \$ 00, \$ 80, \$ 80, \$ 00 \quad$;B

; XX40

FCB $\$ 80, \$ 00, \$ 00, \$ 80, \$ 00, \$ 80, \$ 80, \$ 00$;B

FCB $\$ 00, \$ 80, \$ 80, \$ 00, \$ 80, \$ 00, \$ 00, \$ 80$;A

FCB $\$ 00, \$ 80, \$ 80, \$ 00, \$ 80, \$ 00, \$ 00, \$ 80 \quad ; A$

FCB $\$ 80, \$ 00, \$ 00, \$ 80, \$ 00, \$ 80, \$ 80, \$ 00$;B

; XX60

FCB $\$ 00, \$ 80, \$ 80, \$ 00, \$ 80, \$ 00, \$ 00, \$ 80 \quad ; A$

FCB $\$ 80, \$ 00, \$ 00, \$ 80, \$ 00, \$ 80, \$ 80, \$ 00$;B

FCB $\$ 80, \$ 00, \$ 00, \$ 80, \$ 00, \$ 80, \$ 80, \$ 00$;B

FCB $\$ 00, \$ 80, \$ 80, \$ 00, \$ 80, \$ 00, \$ 00, \$ 80 \quad ; A$

; XX80

FCB $\$ 80, \$ 00, \$ 00, \$ 80, \$ 00, \$ 80, \$ 80, \$ 00$;B

FCB $\$ 00, \$ 80, \$ 80, \$ 00, \$ 80, \$ 00, \$ 00, \$ 80 \quad ; A$

FCB $\$ 00, \$ 80, \$ 80, \$ 00, \$ 80, \$ 00, \$ 00, \$ 80$;A

FCB $\$ 80, \$ 00, \$ 00, \$ 80, \$ 00, \$ 80, \$ 80, \$ 00 \quad$;B

; XXA0

FCB $\$ 00, \$ 80, \$ 80, \$ 00, \$ 80, \$ 00, \$ 00, \$ 80 \quad ; A$

FCB $\$ 80, \$ 00, \$ 00, \$ 80, \$ 00, \$ 80, \$ 80, \$ 00 \quad$;B

FCB $\$ 80, \$ 00, \$ 00, \$ 80, \$ 00, \$ 80, \$ 80, \$ 00$;B

FCB $\$ 00, \$ 80, \$ 80, \$ 00, \$ 80, \$ 00, \$ 00, \$ 80 \quad ; A$

; $\mathrm{XXC0}$

FCB $\$ 00, \$ 80, \$ 80, \$ 00, \$ 80, \$ 00, \$ 00, \$ 80 \quad ; \mathrm{A}$

FCB $\$ 80, \$ 00, \$ 00, \$ 80, \$ 00, \$ 80, \$ 80, \$ 00 \quad$;B

FCB $\$ 80, \$ 00, \$ 00, \$ 80, \$ 00, \$ 80, \$ 80, \$ 00 \quad$;B

FCB $\$ 00, \$ 80, \$ 80, \$ 00, \$ 80, \$ 00, \$ 00, \$ 80 \quad ; A$

; XXE0

FCB $\$ 80, \$ 00, \$ 00, \$ 80, \$ 00, \$ 80, \$ 80, \$ 00 \quad ; A$

FCB $\$ 00, \$ 80, \$ 80, \$ 00, \$ 80, \$ 00, \$ 00, \$ 80 ; B$

FCB $\$ 00, \$ 80, \$ 80, \$ 00, \$ 80, \$ 00, \$ 00, \$ 80 ; B$

FCB $\$ 80, \$ 00, \$ 00, \$ 80, \$ 00, \$ 80, \$ 80, \$ 00 ; \mathrm{A}$

;

; CHARACTER LOOKUP TABLE

CHARTBL: $\quad$ FCB $\$ 41, \$ 42, \$ 43, \$ 44, \$ 45, \$ 46, \$ 47, \$ 00 ; 00-07$

FCB $\$ 00, \$ 00, \$ 00, \$ 00, \$ 00, \$ 00, \$ 00, \$ 00 \quad ; 08-0 F$

FCB $\$ 49, \$ 4 \mathrm{~A}, \$ 4 \mathrm{~B}, \$ 4 \mathrm{C}, \$ 4 \mathrm{D}, \$ 4 \mathrm{E}, \$ 50, \$ 00 ; 10-17$

FCB $\$ 00, \$ 00, \$ 00, \$ 00, \$ 00, \$ 00, \$ 00, \$ 00 \quad ; 18-1 \mathrm{~F}$

FCB $\$ 51, \$ 52, \$ 53, \$ 54, \$ 55, \$ 56, \$ 57, \$ 00 \quad ; 20-27$

FCB $\$ 00, \$ 00, \$ 00, \$ 00, \$ 00, \$ 00, \$ 00, \$ 00 \quad ; 28-2 F$

FCB $\$ 59, \$ 5 \mathrm{~A}, \$ 5 \mathrm{~B}, \$ 5 \mathrm{C}, \$ 5 \mathrm{D}, \$ 5 \mathrm{E}, \$ 5 \mathrm{~F}, \$ 75 ; 30-37$

FCB $\$ 00, \$ 00, \$ 00, \$ 00, \$ 00, \$ 00, \$ 00, \$ 00 ; 38-3 \mathrm{~F}$

FCB $\$ 61, \$ 62, \$ 63, \$ 64, \$ 65, \$ 66, \$ 68, \$ 74 ; 40-47$

FCB $\$ 00, \$ 00, \$ 00, \$ 00, \$ 00, \$ 00, \$ 00, \$ 00 \quad ; 48-4 \mathrm{~F}$

FCB $\$ 69, \$ 6 \mathrm{~A}, \$ 6 \mathrm{~B}, \$ 6 \mathrm{C}, \$ 6 \mathrm{D}, \$ 6 \mathrm{E}, \$ 70, \$ 74 ; 50-57$

FCB $\$ 00, \$ 00, \$ 00, \$ 00, \$ 00, \$ 00, \$ 00, \$ 00 ; 58-5 \mathrm{~F}$

FCB $\$ 71, \$ 72, \$ 73, \$ 74, \$ 75, \$ 76, \$ 77, \$ 72 ; 60-67$

FCB $\$ 00, \$ 00, \$ 00, \$ 00, \$ 00, \$ 00, \$ 00, \$ 00 \quad ; 68-6 \mathrm{~F}$ 


\begin{tabular}{|c|c|c|c|}
\hline \multicolumn{4}{|c|}{; HELP FILE DUMP } \\
\hline \multirow{2}{*}{ SAYHELP: } & LDX & \multirow{2}{*}{\multicolumn{2}{|c|}{$\begin{array}{l}\text { \#HELPFIL } \\
\text { TTYO }\end{array}$}} \\
\hline & BRA & & \\
\hline \multicolumn{4}{|c|}{; A TTY ROUTINE FOR SAIL68 **DO NOT DELETE! USED BY PROMPT ETC!** } \\
\hline \multicolumn{4}{|c|}{-USES \$FD BYTES } \\
\hline$-\mathrm{TO}$ & \multicolumn{3}{|c|}{-TO ENTER FOR XMIT, LDX \#table AND THEN JMP TTYO } \\
\hline$-\mathrm{TO}$ & \multicolumn{3}{|c|}{-TO ENTER IN REC MODE LDX \#table AND JMP TTYR0 } \\
\hline$-\mathrm{MU}$ & \multicolumn{3}{|c|}{-MUST USE EE TO SWITCH FROM SENDING TO REC CHARS } \\
\hline$-\mathrm{CA}$ & \multicolumn{3}{|c|}{-CAN GO BACK TO SENDING W/O PROBLEM } \\
\hline & \multicolumn{3}{|c|}{-ARG TABLE CODES.. } \\
\hline ; & ASCII & \multicolumn{2}{|c|}{-TYPE IT AND GO ON WITH XMIT INTERRUPTS ON } \\
\hline ; & 00 & \multicolumn{2}{|c|}{-BACK TO THE PROMPT } \\
\hline ; & AA aaa: & & O aaaa WITH XMIT INTERRUPTS STILL ON (USE FDB!) \\
\hline ; & 80 aа & \multicolumn{2}{|c|}{-SEND CHAR AT LOC aa } \\
\hline ; & 81 aa & \multicolumn{2}{|c|}{-SEND HEX PAIR AT LOC aa } \\
\hline ; & $\mathrm{EE}$ & \multicolumn{2}{|c|}{-SEND ETX \& WAIT FOR A CHAR (MUST BE FOLLOWED BY 9X) } \\
\hline ; & 90 aa & \multicolumn{2}{|c|}{-REC A CHAR AND SAVE IT AT aa } \\
\hline ; & 91 aa & \multicolumn{2}{|c|}{-REC A HEX PAIR AND SAVE IT AT aa (ABORT TO PROMPT $<>$ HEX) } \\
\hline & 94 aа & \multirow{2}{*}{\multicolumn{2}{|c|}{$\begin{array}{l}\text {-CLEAR SCRTCH } 1,2 \text { \& WAIT FOR A HEX STRING, PUT AT aa,aa+1 } \\
\text { (ENTRY TERMINATED BY SP OR CR) }\end{array}$}} \\
\hline ; & & & \\
\hline TTY: & LDX & XTTY & ;LOAD X FROM STORAGE LOCATION \\
\hline \multirow[t]{5}{*}{ TTYO: } & LDD & \#TTY & ;SET REENTER VECTOR \\
\hline & STD & XVECT & \\
\hline & LDAA & $00, X$ & ;GET ARGUMENT \\
\hline & BNE & TRYAA & \\
\hline & JMP & PROMPT & ;IF =00, GO DIRECTLY TO PROMPT.. \\
\hline \multirow{5}{*}{ TRYAA: } & & \multicolumn{2}{|l|}{ \#\$AA } \\
\hline & BNE & \multirow{2}{*}{\multicolumn{2}{|c|}{ TRYASC }} \\
\hline & INX & & \\
\hline & LDX & $0, \mathrm{X}$ & ;VECTOR TO X \\
\hline & JMP & $0, \mathrm{X}$ & ;AND JUMP TO M(X) \\
\hline \\
\hline TRYASC: & TSTA & & \\
\hline & BMI & TRY80 & ;IF MSB IS 1 , NOT ASCII.. \\
\hline & JSR & LBISX & ;ELSE GET THE ASCII IMM CHAR \\
\hline & TBA & & \\
\hline & JMP & SETOUT & ;GO SEND IT NOW \\
\hline ; & & & \\
\hline TRY80: & CMPA & $\# \$ 80$ & ;REQ IND CHAR? \\
\hline & BNE & TRY81 & \\
\hline & INX & & \\
\hline & JSR & LBISX & ;GET THE ADDRESS (ASSUME BASE PAGE) \\
\hline & JSR & CRADDR & ;CREATE ADDRESS \\
\hline & LDAA & $0, \mathrm{X}$ & ;GET THE IND DATA \\
\hline & JMP & SETOUT & \\
\hline ; & & & \\
\hline TRY81: & CMPA & $\# \$ 81$ & ;REQ IND HEX PAIR? \\
\hline & BNE & TRYEE & \\
\hline
\end{tabular}




\begin{tabular}{|c|c|c|c|}
\hline & INX & & \\
\hline & JSR & LBISX & ;GET THE ADDRESS (ASSUME BASE PAGE) \\
\hline & JSR & CRADDR & ;CREATE ADDRESS \\
\hline & LDD & \#TTY2 & ;THEN SET UP VECT2 EXIT PATH \\
\hline & STD & VECT2 & \\
\hline & LDAA & $0, \mathrm{X}$ & ;GET THE IND DATA NOW \\
\hline & JMP & OUTBYTE & \\
\hline & & & \\
\hline TTY2: & LDD & \#TTY & ;EXIT PATH FOR VECT2 STUFF.. \\
\hline & JMP & XSET & ;(ALLOWS OUTBYTE TO RETURN TO TTY) \\
\hline ; & & & \\
\hline TRYEE: & CMPA & $\#$ \#EE & ;SEND ETX \& GO TO RECEIVE MODE? \\
\hline & BEQ & GOTEE & \\
\hline TRERR: & JMP & PROMPT & ;IF NOT VALID OUT GROUP CONTROL CHAR, JUST \\
\hline $\begin{array}{l}\text { PROMPT } \\
\end{array}$ & & & \\
\hline GOTEE: & INX & & ;SWITCH TO INPUT MODE \\
\hline & STX & XTTY & ;SAVE THE CONTROL TABLE POINTER \\
\hline & LDAA & $\# \$ 03$ & ;SEND ETX \\
\hline & JSR & GENPAR & \\
\hline & STAA & SCDR & \\
\hline & BCLR & SCCR $2, \$ C 0$ & ;STOP FURTHER XMIT INTERRUPTS \\
\hline & LDD & \#TTY3 & ; AND PREPARE TO RECEIVE THE ETX ECHO \\
\hline & JMP & RSET & \\
\hline ; & & & \\
\hline TTY3: & CMPA & $\# \$ 03$ & ;GOT ETX YET? \\
\hline & BEQ & TTY4 & ; KEEP WAITING TILL IT ECHOS \\
\hline & JMP & SCIXIT & \\
\hline ; & & & \\
\hline TTY4: & LDD & \#TTYR & ;WHEN GOT ETX, PREP TO REC DATA CHARS \\
\hline & JMP & RSET & ; SET RVECT TO TTYR AND EXIT \\
\hline TTY REC & ceCtic & ON FNTD & F FPOM COMMAND CHAIN W/O NEW CHAP \\
\hline TTYRO: & STX & XTTY & :JUST SAVE TABLE POINTER \\
\hline & BRA & TTY4 & ; AND GO GET A NEW CHAR \\
\hline ENTER $\mathrm{H}$ & FROM & RVECT WIT & ATA CHAR IN A \\
\hline TTYR: & LDX & XTTY & ; WHILE WE FIGURE OUT WHAT TO DO WITH IT \\
\hline & JSR & LBISX & ;GET THE CONTROL CHAR.. \\
\hline TRY90: & CMPB & $\# \$ 90$ & ;SAVE WHOLE CHAR \\
\hline & BNE & TRY91 & \\
\hline SAVCHR: & JSR & LBISX & ;IF 90, SAVE WHOLE CHAR AT IMM ADDRESS \\
\hline & JSR & CRADDR & ;CREATE ADDRESS \\
\hline & STAA & $0, \mathrm{X}$ & ;SAVE THE DATA BYTE IN M(X) \\
\hline & BRA & CKNEXT & ;CHECK NEXT CONTROL BYTE FOR TYPE (READ OR \\
\hline WRITE) & & & \\
\hline & & & \\
\hline TRY91: & CMPB & $\# \$ 91$ & ;ENTER A HEX PAIR? \\
\hline & BNE & TRY94 & \\
\hline & JSR & ASCHEX & \\
\hline & BCS & TRERR & ;ABORT IF NOT HEX CHAR \\
\hline
\end{tabular}




\begin{tabular}{|c|c|c|c|}
\hline & ASLA & & \\
\hline & ASLA & & ;MOVE OVER \\
\hline & ASLA & & \\
\hline & ASLA & & \\
\hline & STAA & SCRTCH1 & ;SAVE TILL NEXT PASS \\
\hline & LDD & \#TTYHEX & ;PREP FOR 2ND HEX CHAR \\
\hline & JMP & RSET & \\
\hline & & & \\
\hline TTYHEX: & JSR & ASCHEX & ;( SECOND HALF OF TRY91) \\
\hline & BCS & TRERR & ;ABORT IF NOT A VALID HEX CHAR \\
\hline & ORAA & SCRTCH1 & ;COMBINE HIGH AND LOW CHARS \\
\hline & LDX & XTTY & \\
\hline & JSR & LBISX & ;GET SINK ADDRESS \\
\hline & JSR & CRADDR & ;CREATE ADDRESS \\
\hline & STAA & $0, \mathrm{X}$ & \\
\hline & LDD & \#TTYR & ;REPAIR THE VECTOR \\
\hline & STD & RVECT & ; AND ON TO CHECK NEXT COMMAND \\
\hline & BRA & CKNEXT & \\
\hline ; & & & \\
\hline TRY94: & CMPB & $\# \$ 94$ & ;IS IT IN4? \\
\hline & BNE & TRERR & ;QUIT IF NOT A VALID CODE \\
\hline GOT94: & CLR & SCRTCH1 & ;GOT FIRST DIGIT IN A, CLEAR INPUT BUFFER \\
\hline & CLR & SCRTCH2 & \\
\hline & PSHA & & ;SAVE THE ARGUMENT \\
\hline & LDD & \#SAVE4 & \\
\hline & STD & VECT2 & ;PREPARE EXIT VECTOR \\
\hline & LDD & \#IN4HEX & \\
\hline & STD & RVECT & ;CHANGE RVECT \\
\hline & PULA & & \\
\hline & JMP & IN4HEX & ;AND PROCESS THE FIRST DIGIT \\
\hline ; & & & \\
\hline SAVE4: & LDD & \#TTYR & \\
\hline & STD & RVECT & ;REPAIR THE VECTOR \\
\hline & LDX & XTTY & ;GET THE TABLE POINTER \\
\hline & JSR & LBISX & ;AND GET THE SINK ADDRESS \\
\hline & JSR & CRADDR & ;CREATE ADDRESS \\
\hline & LDD & SCRTCH1 & ;AND STORE THE DOUBLE BYTE \\
\hline & STD & $0, \mathrm{X}$ & \\
\hline & BRA & CKNEXT & ;CHECK NEXT CONTROL CHAR \\
\hline CKNEXT: & LDX & XTTY & ;IS THE NEXT CONTROL CHAR AN INP OR OUT? \\
\hline & LDAA & $0, \mathrm{X}$ & ;GET THE CONTROL CHAR \\
\hline & ANDA & \#\$F0 & ; AND CHECK THE HIGH NIBBLE \\
\hline & CMPA & $\# \$ 90$ & ;IS IT AN INPUT TYPE? \\
\hline & BNE & TTYOL & ;F NOT, SWITCH TO OUTPUT MODE \\
\hline & JMP & SCIXIT & ;ELSE GET ANOTHER CHAR \\
\hline ; & & & \\
\hline $\begin{array}{l}\text { TTYOL: } \\
\text {;-- }\end{array}$ & JMP & TTYO & \\
\hline LBISX: & LDAB & $0, \mathrm{X}$ & ;SUBROUTINE TO LOAD B, INC \& STR X \\
\hline & INX & & ; USED BY TTY ABOVE \\
\hline
\end{tabular}




\begin{tabular}{|c|c|c|c|}
\hline \multicolumn{4}{|c|}{ XTTY } \\
\hline \multirow{4}{*}{;-- CRADDR: } & & & \multirow{4}{*}{;SUBROUTINE TO CREATE AN ADDRESS } \\
\hline & LDX & $\# \$ 0000$ & \\
\hline & $\mathrm{ABX}$ & & \\
\hline & RTS & & \\
\hline \multicolumn{4}{|c|}{; AFTER VALID ADDRESS, RECEIVE CONTROL CHAR.. } \\
\hline \multirow[t]{24}{*}{ CONCHAR: } & ANDA & $\# \$ 7 F$ & ;CLEAN OFF FLAG BIT \\
\hline & CMPA & \#!' & ;IS IT '!' \\
\hline & BEQ & SETBG & \\
\hline & CMPA & \#'?' & ;OR IS IT '?' \\
\hline & BEQ & SETQG & \\
\hline & CMPA & \#'O' & ;IS IT 'O', START DATA COLLECTION? \\
\hline & BEQ & TIMEJ & \\
\hline & CMPA & \#'P' & ;IS IT 'P', PAUSE DATA COLLECTION? \\
\hline & BEQ & TIMOUTJ & \\
\hline & CMPA & \#'-' & ;IS IT '-', DATA SCAN REQUEST? \\
\hline & BEQ & DSCANL & \\
\hline & CMPA & \#'A' & ;IS IT'A', SET GAIN CONTROL? \\
\hline & BEQ & ATTCMD & \\
\hline & CMPA & \#' ' & ;SPACE TO ENTER MONITOR? \\
\hline & BEQ & GOMON & \\
\hline & CMPA & $\#$ \$OD & ;CHAR RETURN TO ENTER MONITOR? \\
\hline & BEQ & GOMON & \\
\hline & CMPA & \#'H' & ;HELP? \\
\hline & BEQ & SAYHEL & \\
\hline & CMPA & $\#{ }^{\prime} \mathrm{R}^{\prime}$ & ;RESTART? \\
\hline & BEQ & RSTARTJ & \\
\hline & CMPA & \#'V' & ;ATTEN VALUE REQUEST \\
\hline & BEQ & AVOUTJ & \\
\hline & JMP & SETUNAD & ;UNADDRESS IF NOT VALID.. \\
\hline \multicolumn{4}{|l|}{; } \\
\hline GOMON: & JMP & PROMPT & ;LINK INTO MONITOR \\
\hline SAYHEL: & JMP & SAYHELP & ;LINK TO SAYHELP \\
\hline DSCANL: & JMP & DSCAN & ;LINK TO DATA SCAN \\
\hline TIMEJ: & JMP & TIME & ;LINK TO TIME \\
\hline TIMOUTJ: & JMP & TIMOUT & ;LINK TO TIMOUT \\
\hline RSTARTJ: & JMP & START & ;LINK TO START \\
\hline AVOUTJ: & JMP & AVOUT & ;LINK TO ATTEN VAL OUTPUT \\
\hline \multirow[t]{2}{*}{ SETBG: } & LDD & \#BGROUP & ;PREPARE FOR THE CHAR AFTER ! \\
\hline & JMP & RSET & \\
\hline \multirow[t]{2}{*}{ SETQG: } & LDD & \#QGROUP & ;PREPARE FOR THE CHAR AFTER ? \\
\hline & JMP & RSET & \\
\hline \multirow[t]{2}{*}{ ATTCMD: } & LDD & \#ATTNGRP & ;PREPARE FOR THE CHAR AFTER A \\
\hline & JMP & RSET & \\
\hline \multirow[t]{3}{*}{ BGROUP: } & NOP & & \\
\hline & CMPA & \#'T' & \\
\hline & BEQ & LTESTJ & \\
\hline
\end{tabular}




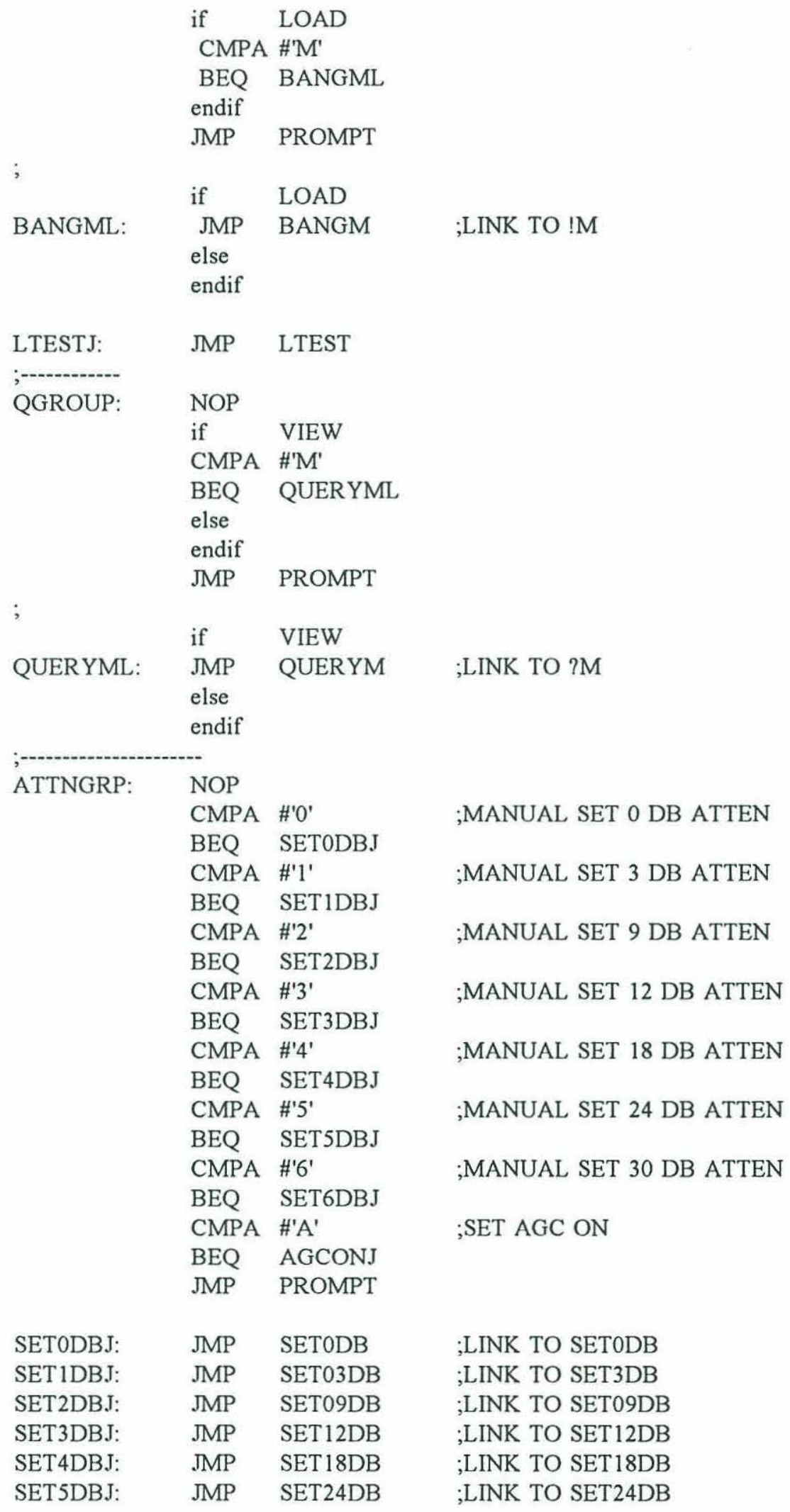




\begin{tabular}{|c|c|c|c|}
\hline $\begin{array}{l}\text { SET6DBJ: } \\
\text { AGCONJ: }\end{array}$ & $\begin{array}{l}\text { JMP } \\
\text { JMP }\end{array}$ & $\begin{array}{l}\text { SET30DB } \\
\text { AGCON }\end{array}$ & $\begin{array}{l}\text {;LINK TO SET30DB } \\
\text {;LINK TO AGCON }\end{array}$ \\
\hline & & & \\
\hline ;PAUSE RE & EST & & STOPS THE DATA DUMP \\
\hline TIMOUT: & CLR & TMSK 1 & ;DISABLE TOC2 INTERUPT \\
\hline & LDAA & $\# \$ F F$ & ;RESET TOC FLAGS \\
\hline & STAA & TFLG1 & \\
\hline & JMP & PROMPT & ;RETURN \\
\hline ;START RE & EST & & TURN ON TIMER INTERUPT \\
\hline TIME: & LDD & TCNT & ;LOAD THE CURRENT TIMER VALUE \\
\hline & ADDD & \#SAMPLE & ;SET AN INITIAL DELAY OF $1.0 \mathrm{mSEC}$ \\
\hline & STD & DTOC2 & \\
\hline & LDAA & $\# \$ 40$ & ;TURN ON OC2 INTERUPT \\
\hline & STAA & TMSK 1 & \\
\hline & JMP & PROMPT & ;RETURN \\
\hline ; DATA SC & & & \\
\hline DSCAN: & LDX & \#DSCANO & \\
\hline & JMP & TTYO & \\
\hline DSCANO: & DB & \$AA & \\
\hline & DW & DSCAN1 & \\
\hline DSCAN1: & BRCLR & TX1FLG,\$01,D & CAN2 ;HAS BUFFER 1 BEEN SENT YET? \\
\hline & BRCLR & TX2FLG, $\$ 01, \mathrm{D}$ & CAN3 ;HAS BUFFER 2 BEEN SENT YET? \\
\hline NOTYET: & LDX & \#NOTRDY & ;WAIT UNTIL A BUFFER IS READY \\
\hline & JMP & TTYO & \\
\hline NOTRDY: & FCB & $\$ 00, \$ 00$ & \\
\hline i & BSET & TXIFLG \$01 & SET BUFFER I TX'D FLAG \\
\hline DSCAN2: & LDX & \#MAXBUF1 & ;SET BUFFER I IXD FLAG \\
\hline & BRA & DSCAN4 & \\
\hline DSCAN3: & BSET & TX2FLG,\$01 & ;SET BUFFER 2 TX'D FLAG \\
\hline & LDX & \#MAXBUF2 & \\
\hline DSCAN4: & STX & TXBUFF & ;SAVE THE BUFFER TO TX IN TXBUFF \\
\hline & LDAB & MAXNUM & \\
\hline & $\mathrm{ABX}$ & & \\
\hline & STX & TXBFULL & ;GENERATE THE BUFFER DONE VALUE \\
\hline ; & LDY & TXBUFF & \\
\hline & STY & TXINT & \\
\hline DSCAN5: & LDAA & $\$ 00, Y$ & ;GET THE DATA POINT HI BYTE \\
\hline & STAA & TXMHI & ;STORE IN BASE PAGE \\
\hline & LDAA & $\$ 01, Y$ & ;GET THE DATA POINT LOW BYTE \\
\hline & STAA & TXMLO & ;STORE IN BASE PAGE \\
\hline & LDAA & $\$ 10, Y$ & ;GET THE INDEX HI BYTE \\
\hline & STAA & TXIHI & \\
\hline & LDAA & $\$ 11, Y$ & ;GET THE INDEX LOW BYTE \\
\hline & STAA & TXILO & \\
\hline ; & LDX & \#BITSND1 & ;PREP FOR TX \\
\hline
\end{tabular}




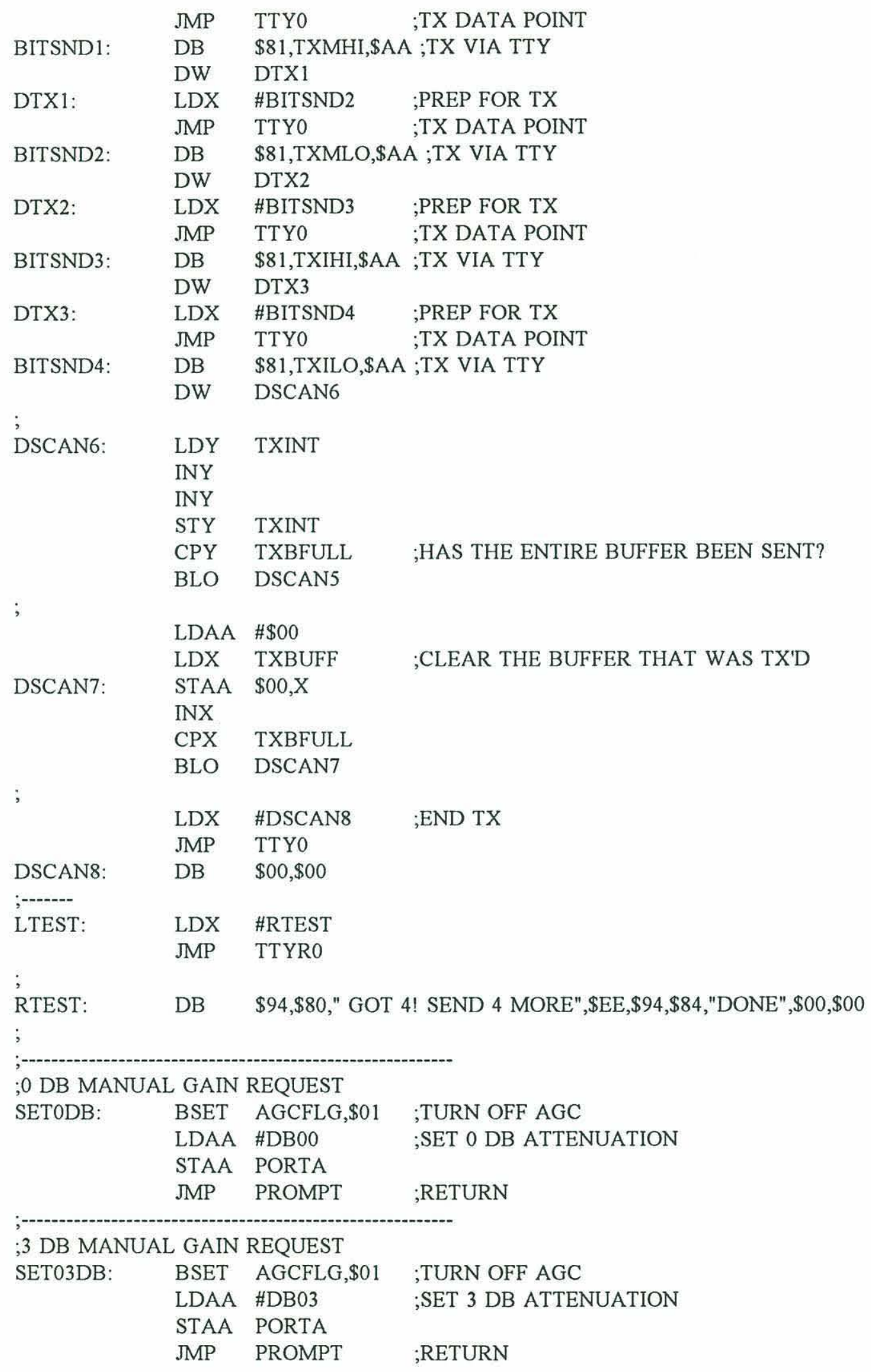




\begin{tabular}{|c|c|c|c|}
\hline \multicolumn{4}{|c|}{;9 DB MANUAL GAIN REQUEST } \\
\hline \multirow[t]{4}{*}{ SET09DB: } & BSET & AGCFLG,\$01 & ;TURN OFF AGC \\
\hline & LDAA & \#DB09 & ;SET 9 DB ATTENUATION \\
\hline & STAA & PORTA & \\
\hline & JMP & PROMPT & ;RETURN \\
\hline \multirow{2}{*}{\multicolumn{4}{|c|}{;12 DB MANUAL GAIN REQUEST }} \\
\hline & & & \\
\hline \multirow[t]{4}{*}{ SET12DB: } & BSET & AGCFLG,\$01 & ;TURN OFF AGC \\
\hline & LDAA & \#DB12 & ;SET 12 DB ATTENUATION \\
\hline & STAA & PORTA & \\
\hline & JMP & PROMPT & ;RETURN \\
\hline \multicolumn{4}{|c|}{;18 DB MANUAL GAIN REQUEST } \\
\hline \multirow[t]{4}{*}{ SET18DB: } & BSET & AGCFLG,\$01 & ;TURN OFF AGC \\
\hline & LDAA & \#DB18 & ;SET 18 DB ATTENUATION \\
\hline & STAA & PORTA & \\
\hline & JMP & PROMPT & ;RETURN \\
\hline \multicolumn{4}{|c|}{;24 DB MANUAL GAIN REQUEST } \\
\hline \multirow[t]{4}{*}{ SET24DB: } & BSET & AGCFLG,\$01 & ;TURN OFF AGC \\
\hline & LDAA & \#DB24 & ;SET 24 DB ATTENUATION \\
\hline & STAA & PORTA & \\
\hline & JMP & PROMPT & ;RETURN \\
\hline \multicolumn{4}{|c|}{;30 DB MANUAL GAIN REQUEST } \\
\hline \multirow[t]{4}{*}{ SET30DB: } & BSET & AGCFLG,\$01 & ;TURN OFF AGC \\
\hline & LDAA & \#DB30 & ;SET 30 DB ATTENUATION \\
\hline & STAA & PORTA & \\
\hline & JMP & PROMPT & ;RETURN \\
\hline \multicolumn{4}{|c|}{;AGC ON REQUEST } \\
\hline \multirow[t]{2}{*}{ AGCON: } & CLR & AGCFLG & ;SET AGC ON FLAG TO 0 (TURN AGC ON) \\
\hline & JMP & PROMPT & ;RETURN \\
\hline \multirow{2}{*}{ AVOUT: } & LDX & \#AVODAT & \\
\hline & JMP & TTY0 & \\
\hline \multirow{2}{*}{ AVODAT: } & DB & $\$ 81$, PORTA, $\$ 0$ & $\$ 00$ \\
\hline & JMP & PROMPT & \\
\hline \multicolumn{4}{|c|}{ 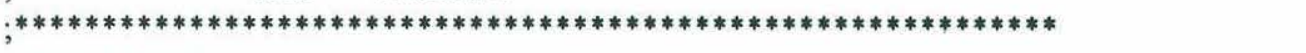 } \\
\hline \multicolumn{4}{|c|}{; HELP FILE } \\
\hline \multirow[t]{10}{*}{ HELPFIL } & $: \mathrm{FCB}$ & \multicolumn{2}{|c|}{ 'elp SAIL DRIVER Mon, Jun 7, 1993, 11:53',\$0D,\$0A } \\
\hline & FCB & \multicolumn{2}{|c|}{ 'Monitor Commands (enter via_or? or !)',\$0D,\$0A } \\
\hline & $\mathrm{FCB}$ & \multicolumn{2}{|c|}{ '_ (space) enter monitor*',\$OD,\$OA } \\
\hline & FCB & \multicolumn{2}{|c|}{ '?Maaaa_llllcr *',\$0D,\$0A } \\
\hline & $\mathrm{FCB}$ & \multicolumn{2}{|c|}{ '!Maaaa_dd...cr *',\$0D,\$0A } \\
\hline & FCB & \multicolumn{2}{|c|}{ 'Attenuator Commands (enter via A)',\$0D,\$0A } \\
\hline & FCB & \multicolumn{2}{|c|}{ ' A0 sets $0 \mathrm{~dB}$ manual attenuation',\$0D, $\$ 0 \mathrm{~A}$} \\
\hline & $\mathrm{FCB}$ & \multicolumn{2}{|c|}{ ' A1 sets $3 \mathrm{~dB}$ manual attenuation',\$0D,\$0A } \\
\hline & FCB & ' $\mathrm{A} 2$ sets $9 \mathrm{~dB}$ & manual attenuation',\$OD,\$0A \\
\hline & FCB & ' $\mathrm{A} 3$ sets $12 \mathrm{dE}$ & manual attenuation', $\$ 0 \mathrm{D}, \$ 0 \mathrm{~A}$ \\
\hline
\end{tabular}




\begin{tabular}{|c|c|c|}
\hline & & $1 \mathrm{~A} 4$ cetc $18 \mathrm{dR}$ manual attenuation' $80 \mathrm{D} \& \mathrm{~A}$ \\
\hline & $\mathrm{FCB}$ & - A5 sets $24 \mathrm{~dB}$ manul attenuation',\$0D, \$0A \\
\hline & FCB & ' A6 sets $30 \mathrm{~dB}$ manual attenuation',\$0D,\$0A \\
\hline & FCB & ' AA turns $\mathrm{AGC}$ on',\$OD, $\$ 0 \mathrm{~A}$ \\
\hline & FCB & 'O starts receiver collecting samples',\$0D,\$0A \\
\hline & FCB & 'P pauses receiver',\$0D,\$0A \\
\hline & FCB & 'R resets the system',\$0D,\$0A \\
\hline & FCB & 'with all RCA UT4 conventions',\$0D,\$0A \\
\hline & $\mathrm{FCB}$ & '* returns prompt',\$00 \\
\hline$; * * * * * * * *$ & $* * * * *$ & $* * * * * * * * * * * * * * * * * * * * * * * * * * * * * * * * *$ \\
\hline & ORG & FFD6 \\
\hline VSCI: & FDB & SCI \\
\hline VSPI: & FDB & START \\
\hline VPAIE: & FDB & START \\
\hline VPAO: & FDB & START \\
\hline VTOF: & FDB & START \\
\hline VTOC5: & FDB & START \\
\hline VTOC4: & FDB & START \\
\hline VTOC3: & FDB & START \\
\hline VTOC2: & FDB & $\mathrm{OC} 2$ \\
\hline VTOC1: & FDB & START \\
\hline VTIC3: & FDB & START \\
\hline VTIC2: & FDB & START \\
\hline VTIC1: & FDB & START \\
\hline VRTI: & FDB & START \\
\hline VIRQ: & FDB & START \\
\hline VXIRQ: & FDB & START \\
\hline VSWI: & FDB & START \\
\hline VILLOP: & FDB & START \\
\hline VCOP: & FDB & START \\
\hline VCLM: & FDB & START \\
\hline VRST: & FDB & START \\
\hline & END & \\
\hline
\end{tabular}




\section{Appendix 3}

This appendix contains the $\mathrm{C}$ source code use by the Macintosh computer for the algorithm of figure 2-4. The user interface follows the guidelines specified by Apple, and the Event Manager is used to initiate all functions. After the hardware is initialized, the program waits in the EventLoop until an event is received. If no events are received in the loop cycle time, the program requests data from the $68 \mathrm{HC} 11$ and the GPS receiver. If the data is available, it is stored to form experiment vectors.

Once enough experiment vectors are collected, the maximum likelihood estimate is calculated. The program the returns to the EventLoop.

\section{TRACKER.C}

Dennis Michael Wojcik

15 July 1993

This program is version 1.0 of the Precision Tracking Receiver. It is tailored for the scenario of a stationary acoustic beacon and a moving observer. The program assumes that the observer has a GPS receiver for positioning data that can produce a fix at least every 2 seconds. If a different navigation system is used to provide ship locations, it is up to the user to provide the proper position format:

\section{?L HHMMSS,DDMM.hh,N,DDDMM.hh,E,x,x,DOP,xxx,M,xxxxx,M}

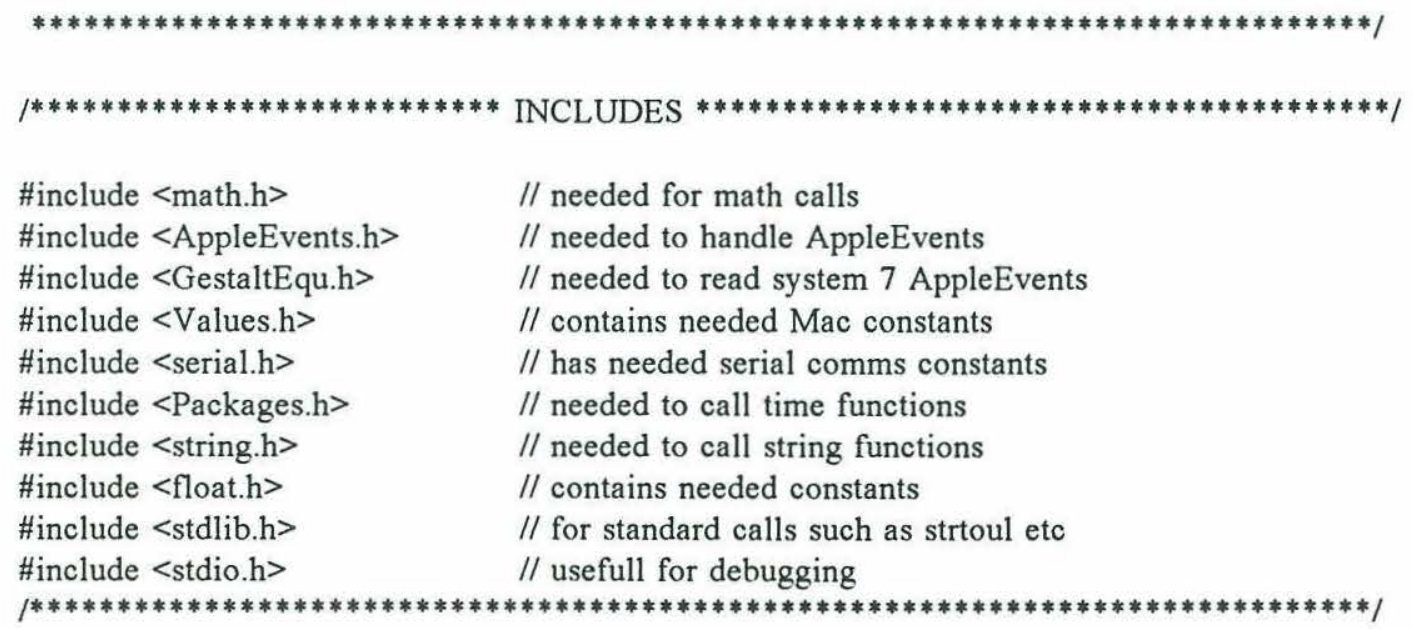




\begin{tabular}{|c|c|c|}
\hline \#define kBaseResID & 128 & // define general resource infor \\
\hline \#define kMoveToFront & (WindowPtr)-1L & \\
\hline \#define kUseDefaultProc & $($ void $*)-1 L$ & \\
\hline \#define kSleep & $1 \mathrm{~L}$ & \\
\hline \#define kLeaveWhereItIs & false & \\
\hline \#define mApple & kBaseResID & // Apple menu resource ID \\
\hline \#define iAbout & 1 & \\
\hline \#define mFile & kBaseResID+1 & // File menu resource ID \\
\hline \#define iQuit & 1 & \\
\hline \#define mAttenuator & kBaseResID+3 & // Attenuator menu resource ID \\
\hline \#define iAuto & 1 & \\
\hline$\#$ define i0dB & 2 & \\
\hline \#define i3dB & 3 & \\
\hline \#define i9dB & 4 & \\
\hline \#define i12dB & 5 & \\
\hline \#define il $8 \mathrm{~dB}$ & 6 & \\
\hline \#define i24dB & 7 & \\
\hline \#define i30dB & 8 & \\
\hline \#define mBaud & kBaseResID+4 & // Baud Rate menu resource ID \\
\hline \#define $\mathrm{i} 300$ & 1 & \\
\hline \#define 1600 & 2 & \\
\hline \#define i1200 & 3 & \\
\hline \#define i2400 & 4 & \\
\hline \#define i4800 & 5 & \\
\hline \#define i 9600 & 6 & \\
\hline \#define mSignal & kBaseResID +5 & // Signal menu resource ID \\
\hline \#define iPeriod & 1 & \\
\hline \#define iNumMax & 2 & \\
\hline \#define iWindow & 3 & \\
\hline \#define iMessageText & 4 & \\
\hline \#define kPeriodDialog & kBaseResID & \\
\hline \#define kNumMaxDialog & kBaseResID+1 & \\
\hline \#define $\mathrm{kWindowDialog}$ & kBaseResID+2 & \\
\hline \#define kLeftMargin & 4 & // define screen margins \\
\hline \#define $\mathrm{kF}$ ontSize & 10 & \\
\hline \#define kRowHeight & $(\mathrm{kFontSize}+2)$ & \\
\hline \#define kDataScanHeight & 1 & \\
\hline \#define kHorizontalOffset & 0 & \\
\hline \#define kGestaltMask & $1 \mathrm{~L}$ & \\
\hline \#define kHorizontalPix & 30 & \\
\hline \#define kVerticalPix & 40 & \\
\hline \#define kMinWindowHeight & 50 & \\
\hline \#define kMinWindowWidth & 80 & \\
\hline \#define kMinTextPosition & 0 & \\
\hline
\end{tabular}




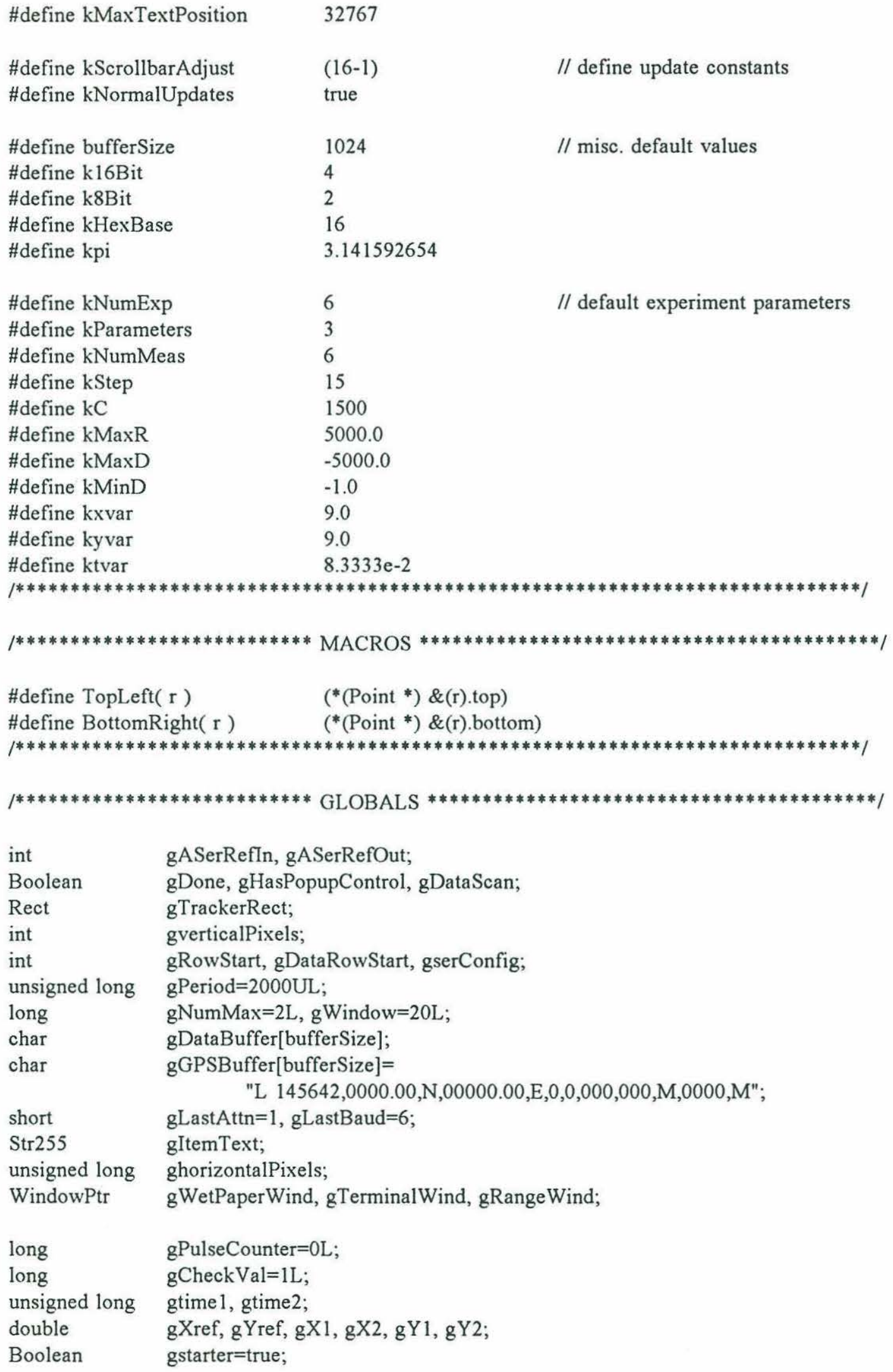




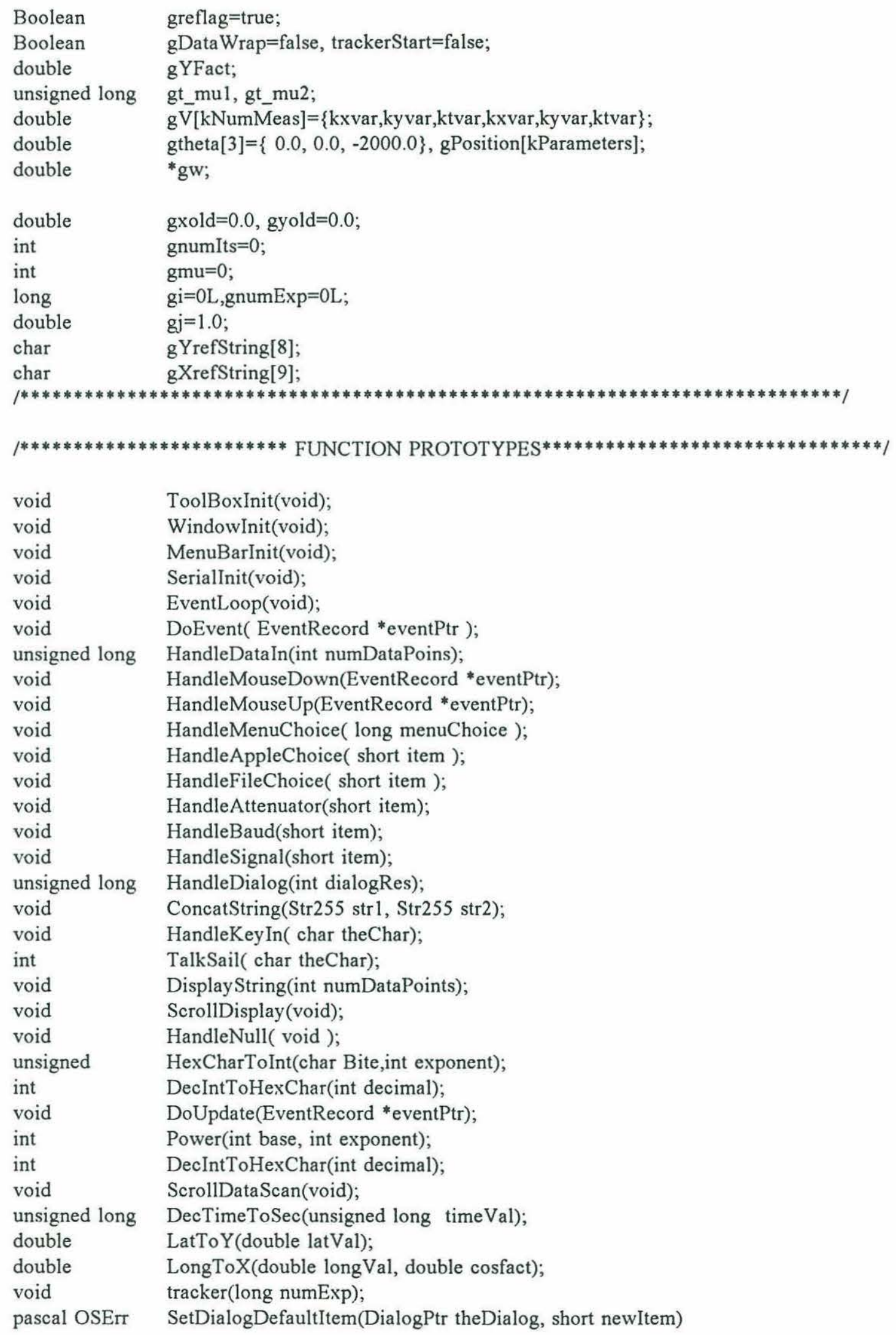




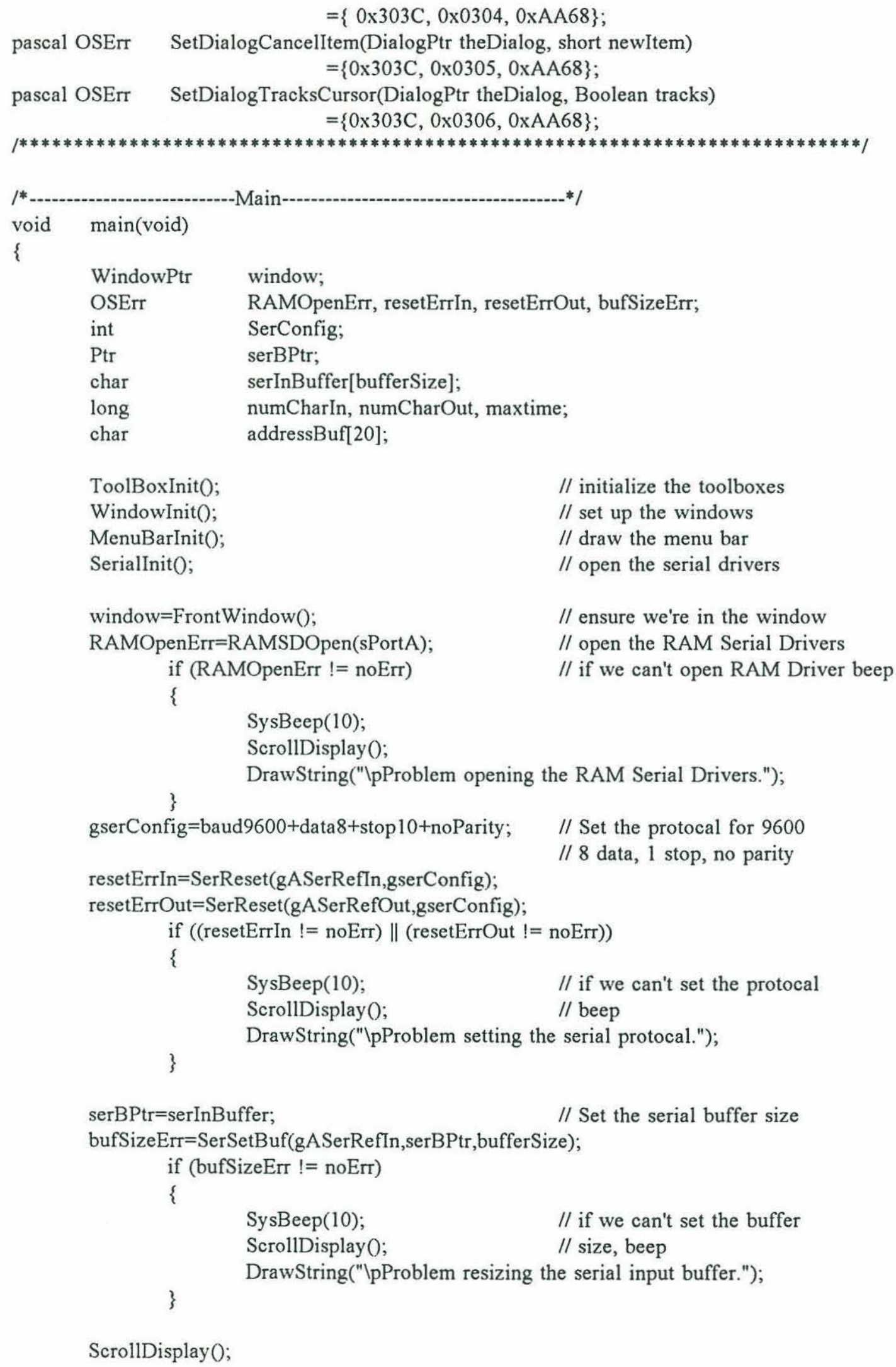


numCharOut $=5 \mathrm{~L}$;

// see if we can talk to the GPS receiver

FSWrite(gASerRefOut, \&numCharOut, "\#GPS ");

SerGetBuf(gASerRefIn,\&numCharIn);

maxtime $=$ TickCount $(\mathrm{O}+20 \mathrm{~L}$;

while(numCharIn < 9L)

\{

SerGetBuf(gASerRefIn,\&numCharIn);

if(TickCount ()$>$ maxtime)

\{

SysBeep(10);

DrawString("'p Can't talk to the GPS reciever, unable to run!!");

ScrollDisplay();

\}

break;

\}

FSRead(gASerRefIn,\&numCharIn,\&addressBuf);

numCharOut $=5 \mathrm{~L}$;

// see if we can talk to the PTR receiver

FSWrite(gASerRefOut, \&numCharOut, "\#PTR ");

SerGetBuf(gASerRefIn,\&numCharIn);

maxtime $=$ TickCount $(\mathrm{O}+20 \mathrm{~L}$;

while(numCharIn < 9L)

\{

SerGetBuf(gASerRefIn,\&numCharIn);

if(TickCount) $>$ maxtime)

\{

SysBeep(10);

DrawString("\p Can't talk to the PTR reciever, unable to run!!");

ScrollDisplay();

maxtime $=$ TickCount ()$^{+120 L \text {; }}$

while(TickCount ()$<\operatorname{maxtime})$

abort);

TickCount () ;

\}

\}

FSRead(gASerRefIn,\&numCharIn,\&addressBuf);

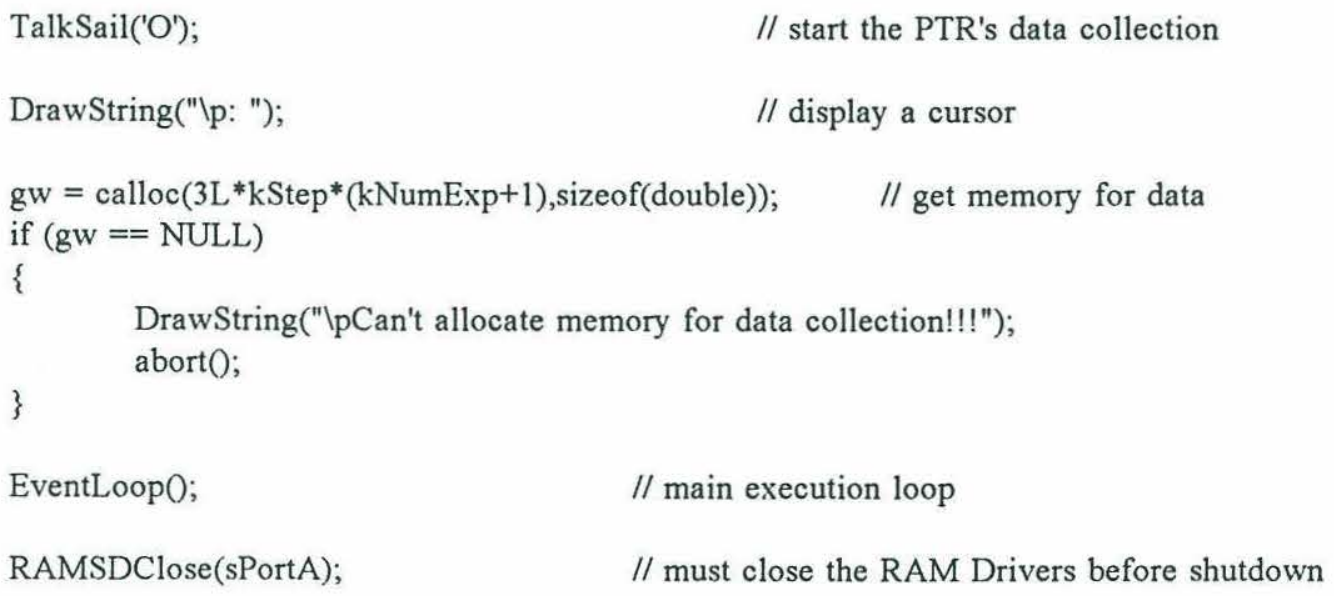




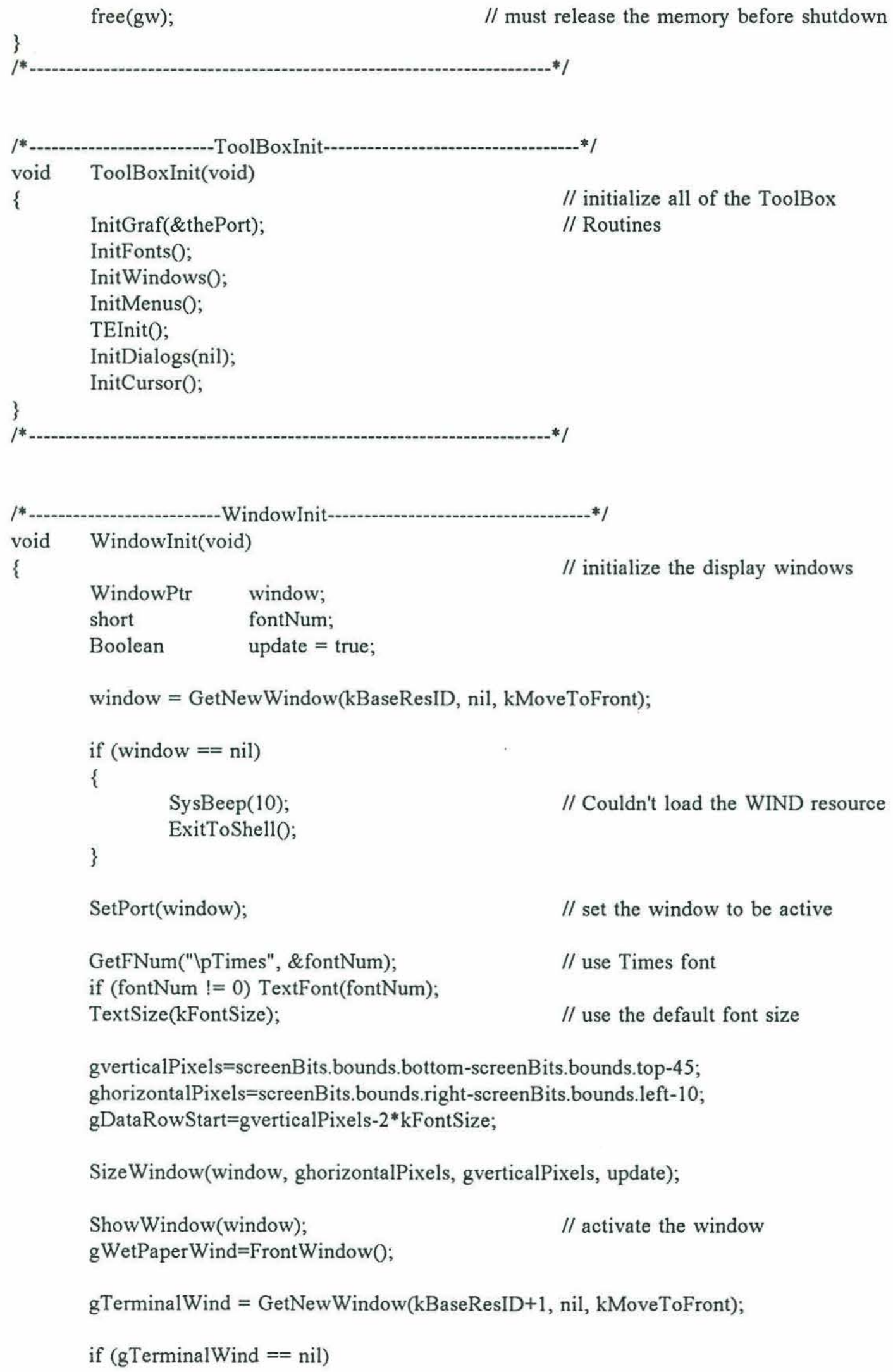




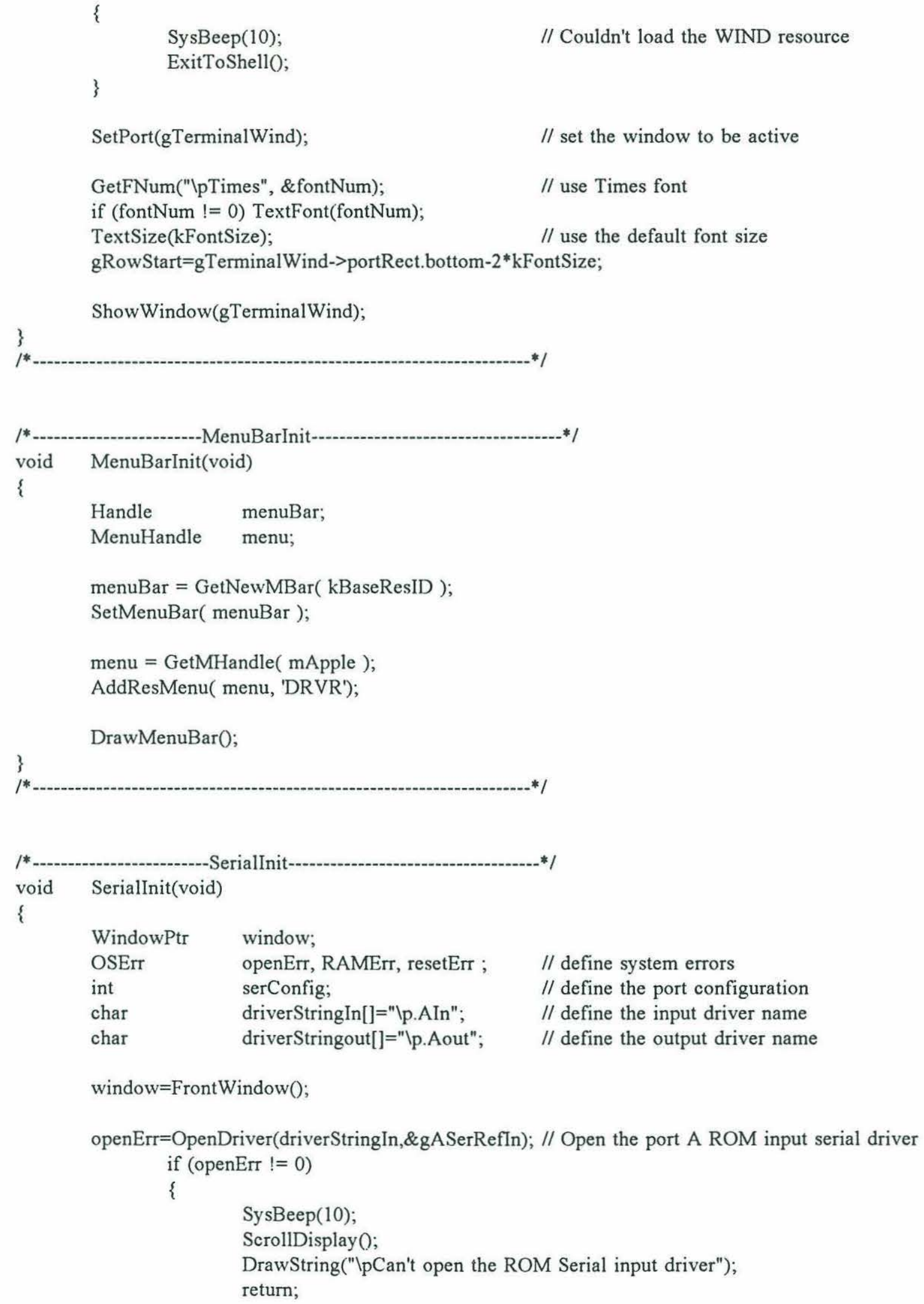


\}

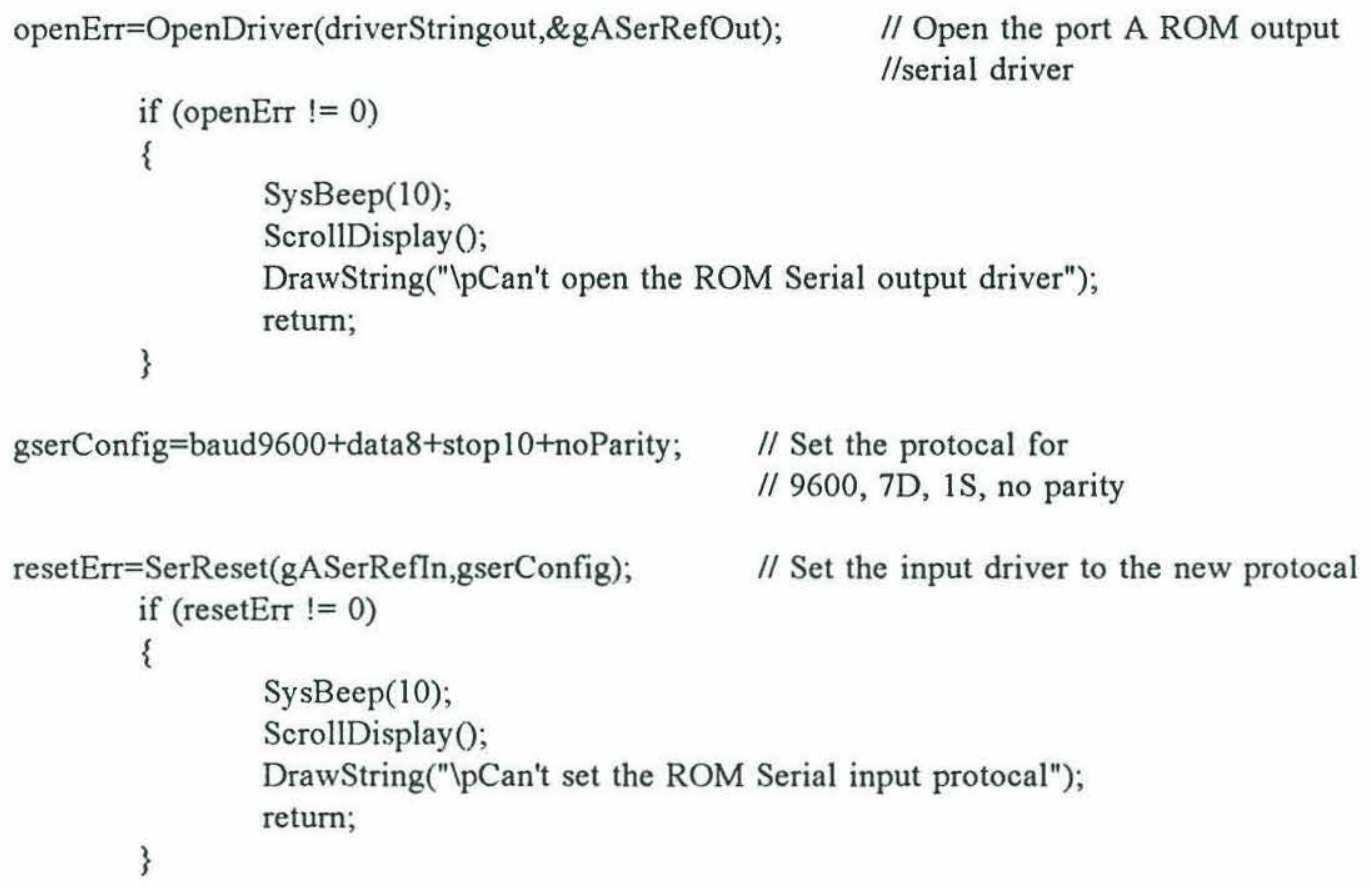




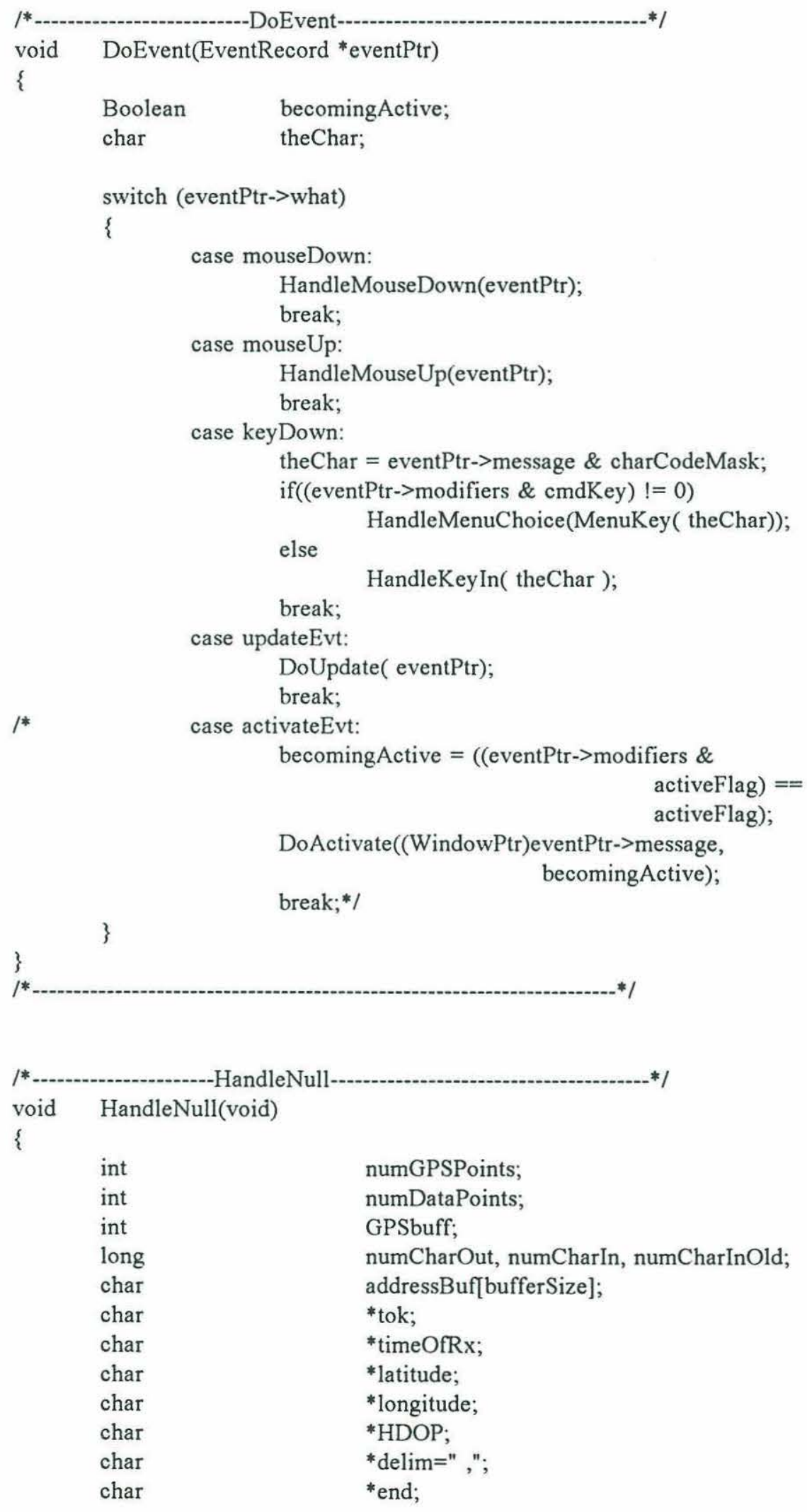




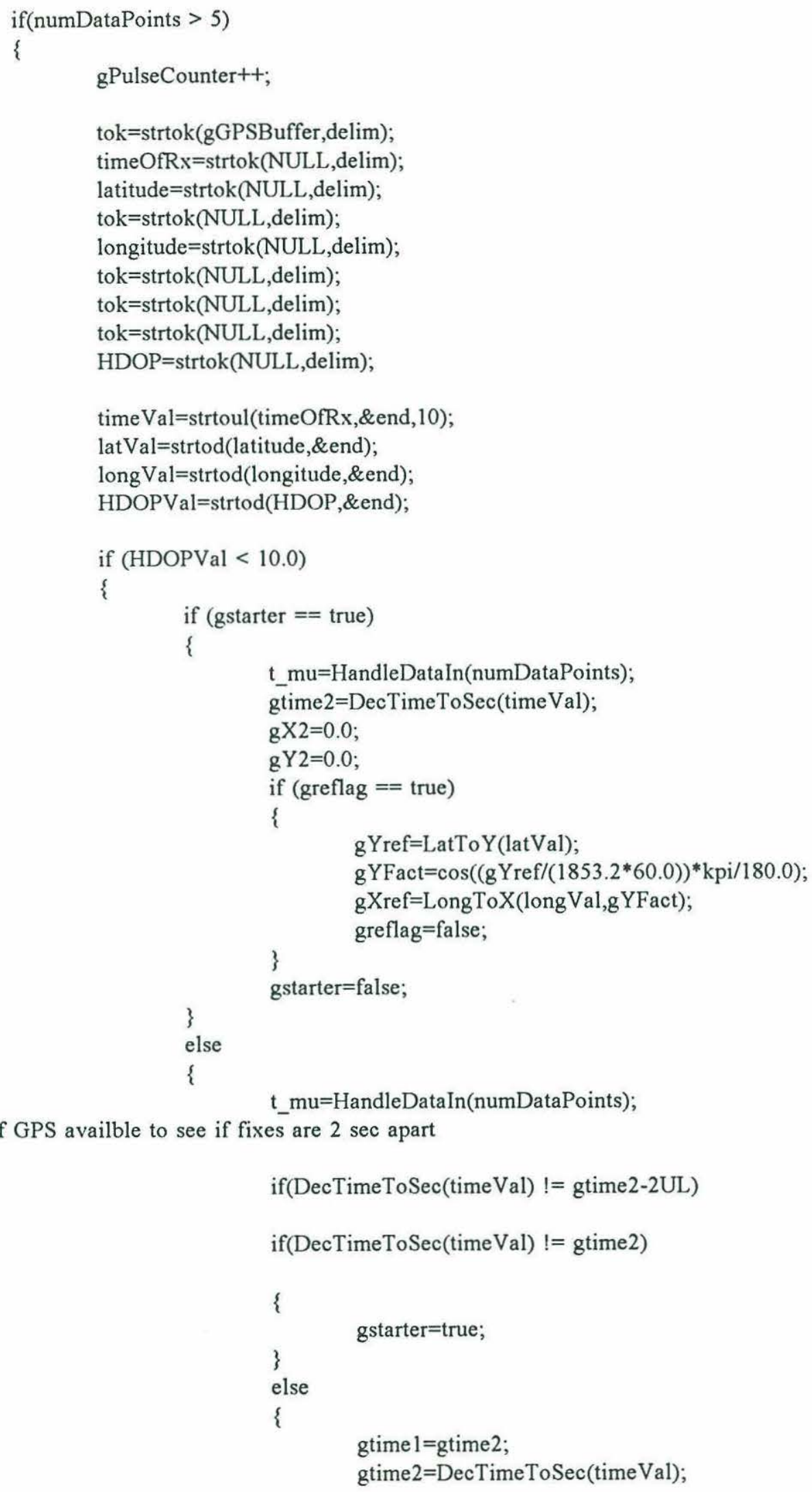


$\mathrm{gX} 1=\mathrm{gX} 2$;

gX2=LongToX(longVal,gYFact)-gXref;

$\mathrm{gY} 1=\mathrm{gY} 2$;

gY2=LatToY(latVal)-gYref;

//---> make up data to test program

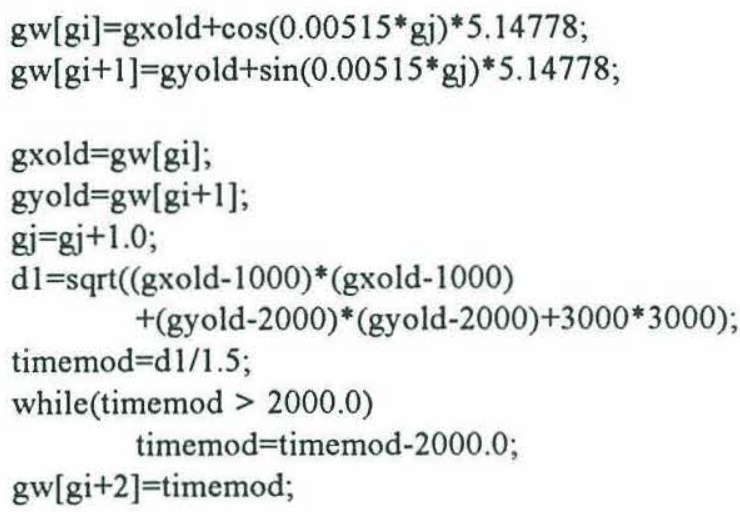

//---> end of simulated data

$/^{*}$-.-> use this if GPS available

*/

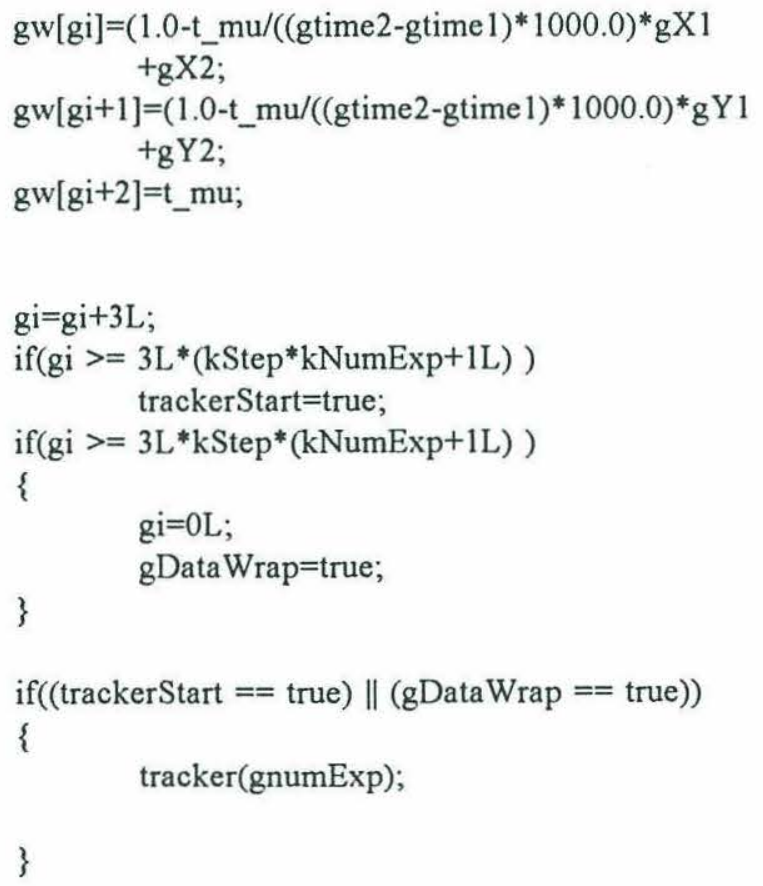




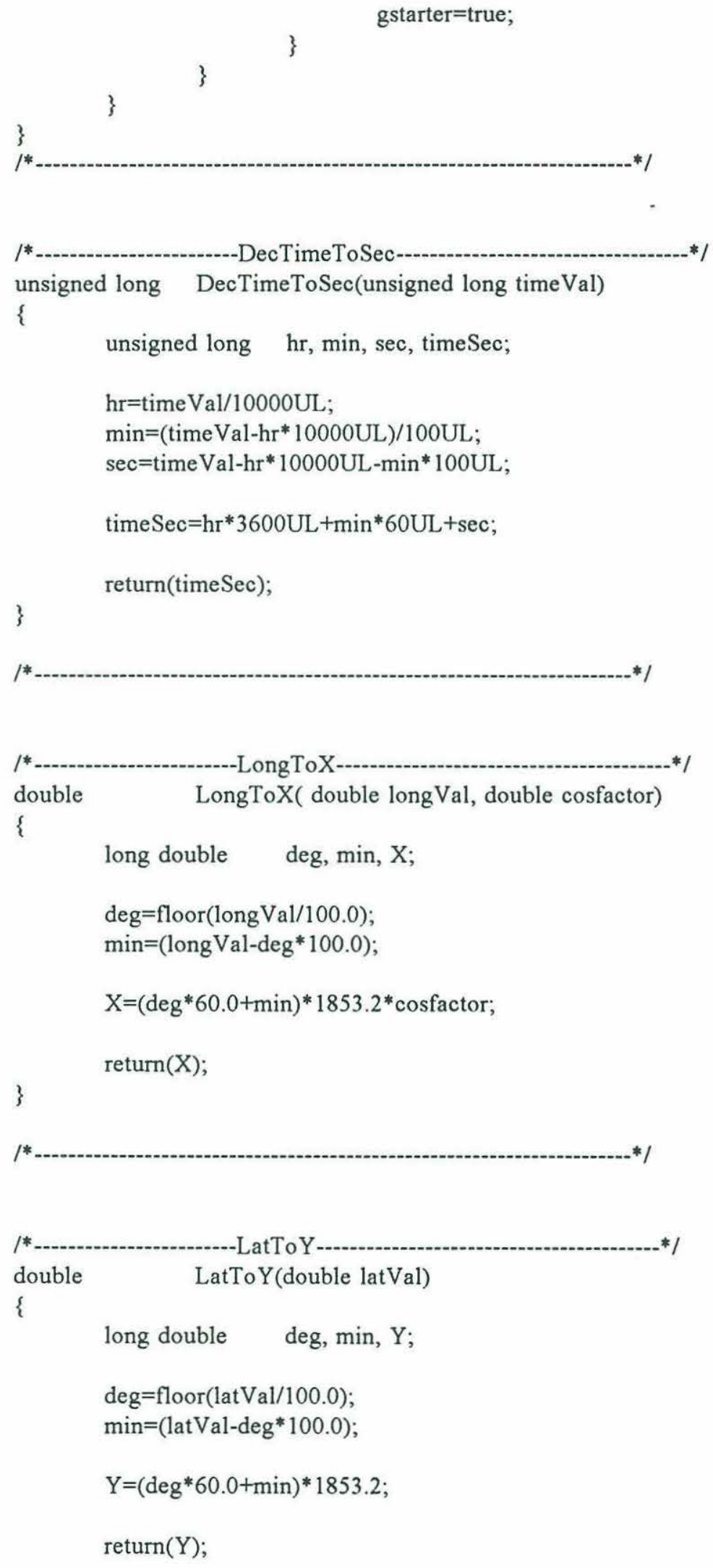



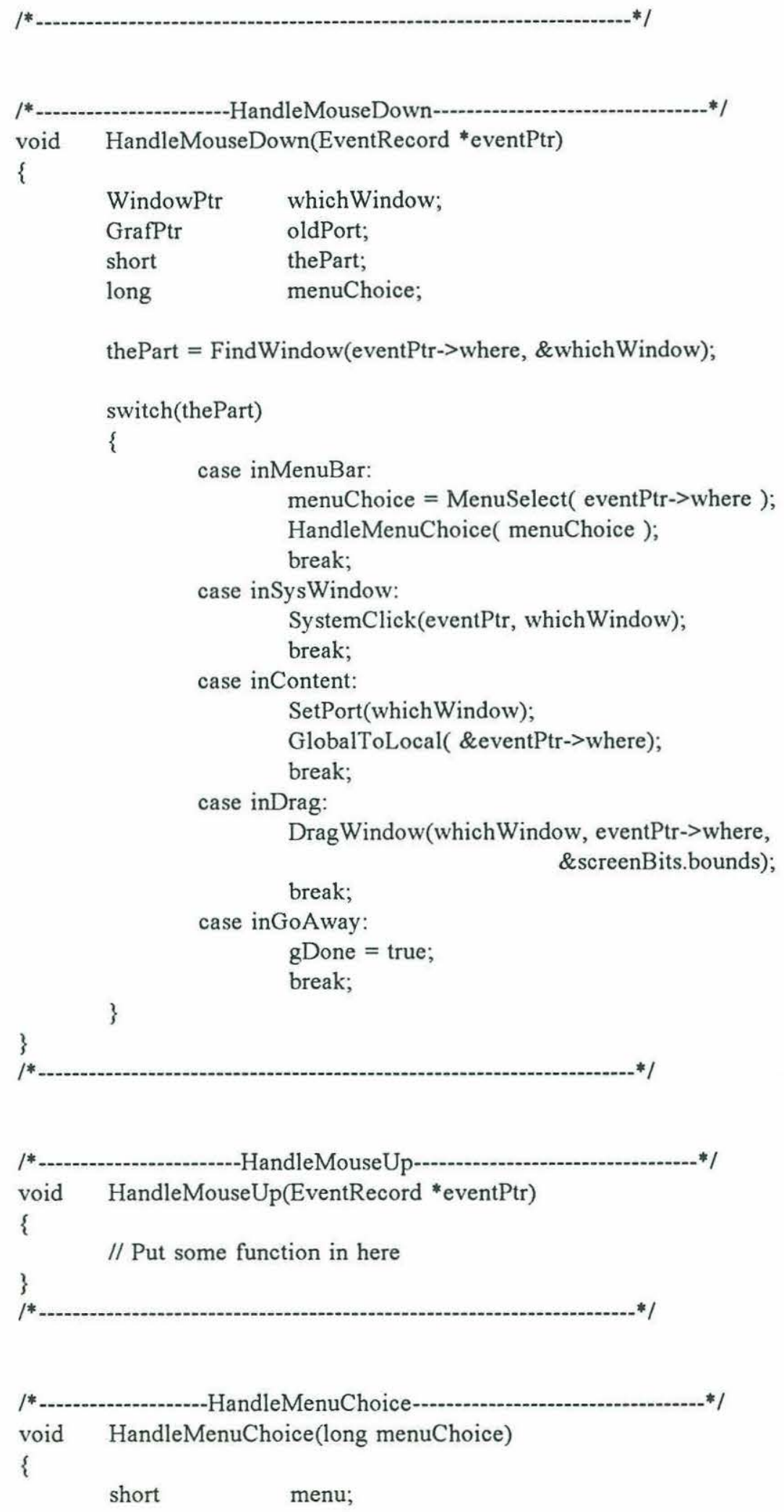


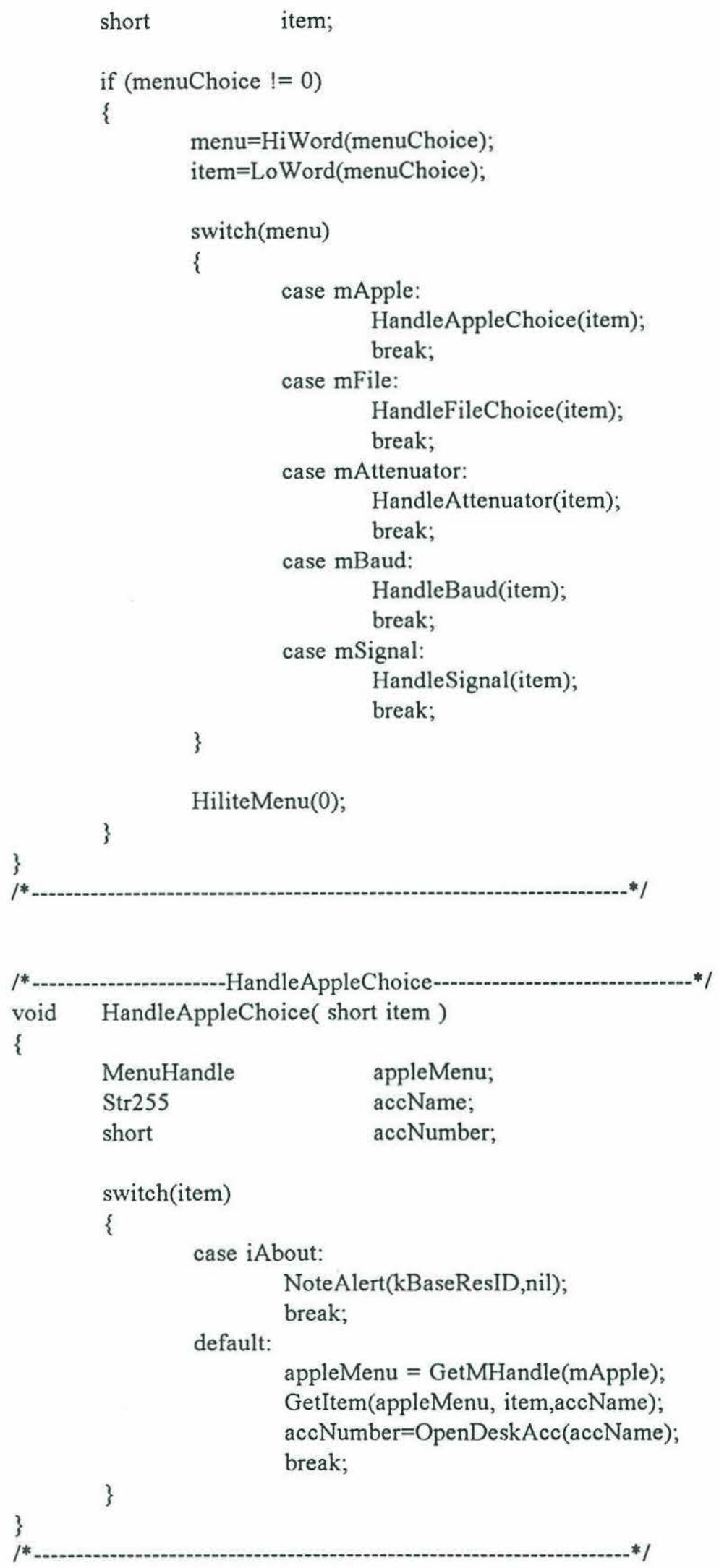




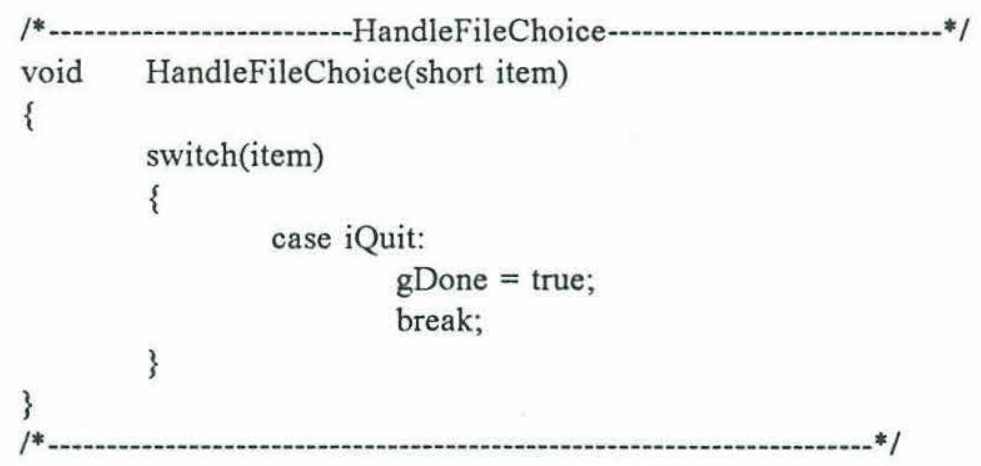




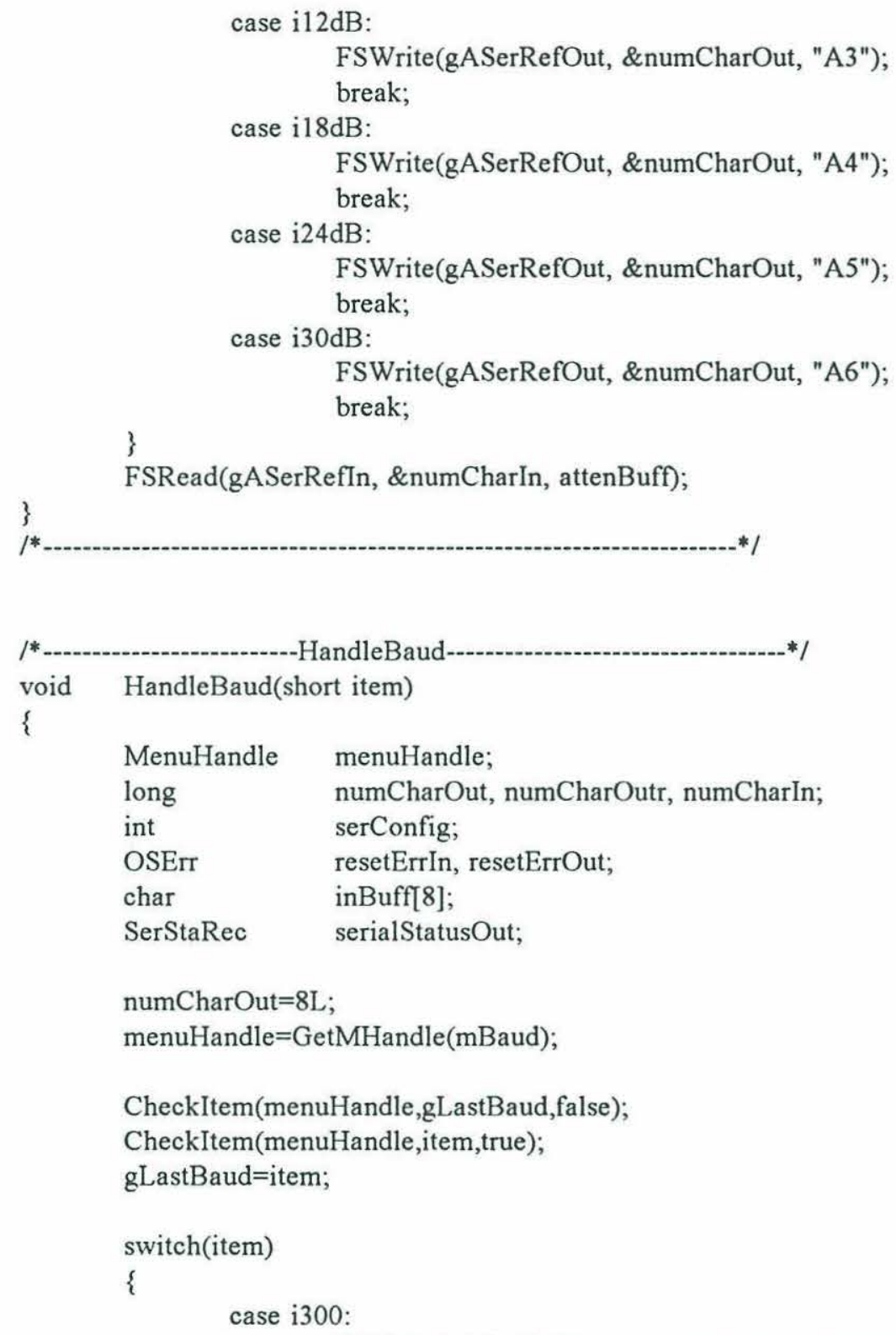

FSWrite(gASerRefOut, \&numCharOut, "\#0000600"); gserConfig=baud $600+$ data $8+$ stop $10+$ noParity; break;

case i1200:

FSWrite(gASerRefOut, \&numCharOut, "\#0001200"); gserConfig=baud $1200+$ data $8+$ stop $10+$ noParity; break;

case i2400:

FSWrite(gASerRefOut, \&numCharOut, "\#0002400"); gserConfig=baud $2400+$ data $8+$ stop $10+$ noParity; 


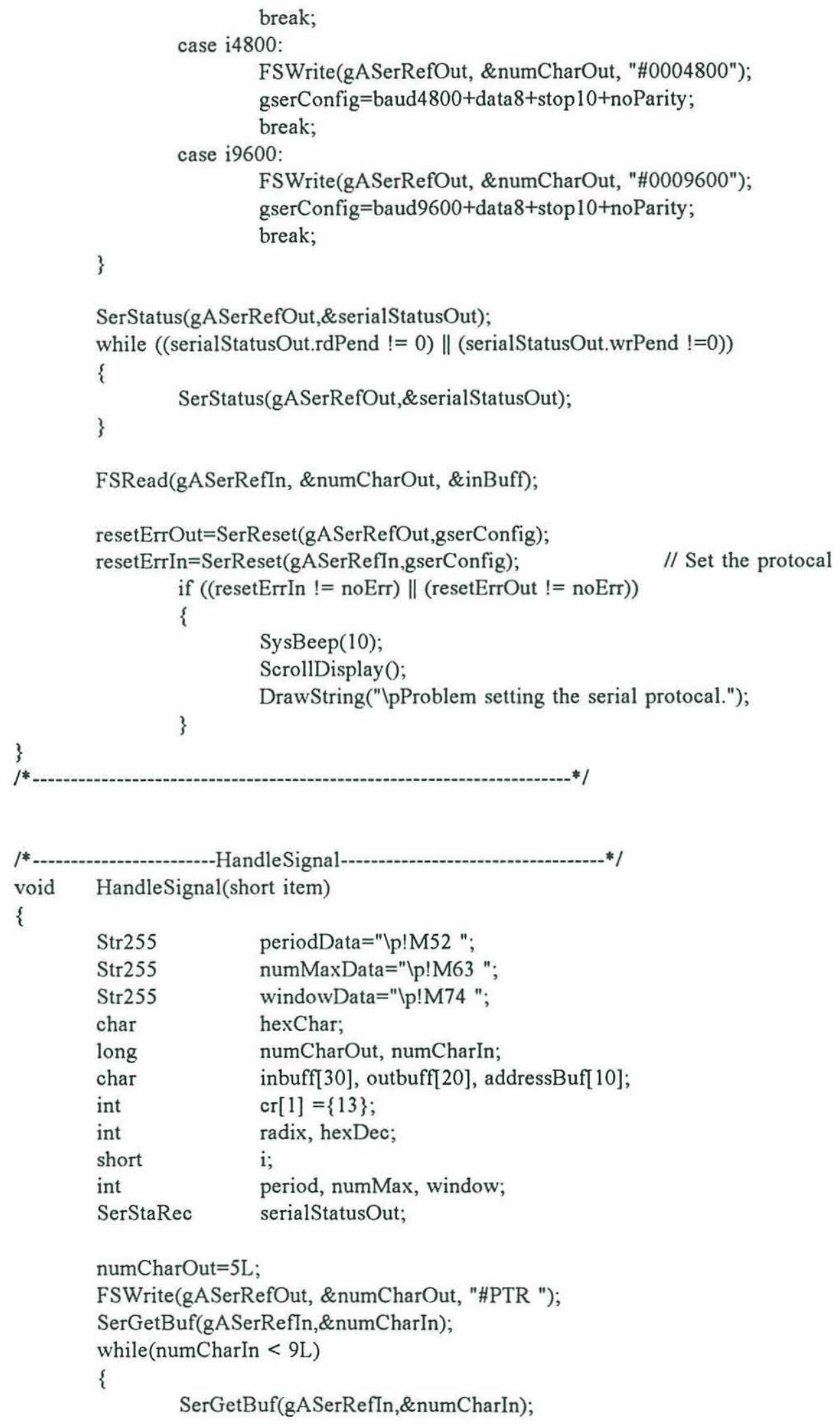


FSRead(gASerRefIn,\&numCharIn,\&addressBuf);

switch(item)

\{

case iPeriod:

gPeriod=HandleDialog(kPeriodDialog);

period $=\mathrm{gPeriod}$;

for $(\mathrm{i}=0 ; \mathrm{i}<\mathrm{k} 16 \mathrm{Bit} ; \mathrm{i}++)$

\{

radix $=$ Power(kHexBase,k16Bit-1-i);

hexDec=period/radix; hexChar=DecIntToHexChar(hexDec); periodData[ $1+\mathrm{i}+$ periodData[0]]=hexChar; period=period-hexDec*radix;

\}

periodData[periodData $[0]+\mathrm{k} 16 \mathrm{Bit}+1]=\mathrm{cr}[0]$;

periodData[0] $=$ periodData[0]+k16Bit+1;

for $(\mathrm{i}=0 ; \mathrm{i}<$ periodData $[0] ; \mathrm{i}++)$

\{

\}

outbuff $[\mathrm{i}]=$ periodData[i+1];

numCharOut=periodData[0];

break;

case iNumMax:

gNumMax=HandleDialog(kNumMaxDialog);

numMax $=$ gNumMax $* 2$;

for $(\mathrm{i}=0 ; \mathrm{i}<\mathrm{k8Bit}$; $\mathrm{i}++)$

\{

radix=Power(kHexBase,k8Bit-1-i);

hexDec=numMax/radix;

hexChar=DecIntToHexChar(hexDec);

numMaxData[ $1+\mathrm{i}+$ numMaxData[0]]=hexChar; numMax=numMax-hexDec*radix;

\}

numMaxData[numMaxData[0] $+\mathrm{k} 8 \mathrm{Bit}+1]=\mathrm{cr}[0]$;

numMaxData[0]=numMaxData[0]+k8Bit+1;

for $(\mathrm{i}=0 ; \mathrm{i}<$ numMaxData[0];i++)

\{

\}

outbuff[i]=numMaxData[i+1];

numCharOut=numMaxData[0];

break;

case iWindow:

$\mathrm{gWindow}=$ HandleDialog(kWindowDialog);

window $=\mathrm{gWindow}$;

for $(\mathrm{i}=0 ; \mathrm{i}<\mathrm{k} 16 \mathrm{Bit} ; \mathrm{i}++)$

\{

radix=Power(kHexBase,k16Bit-1-i);

hexDec=window/radix; 


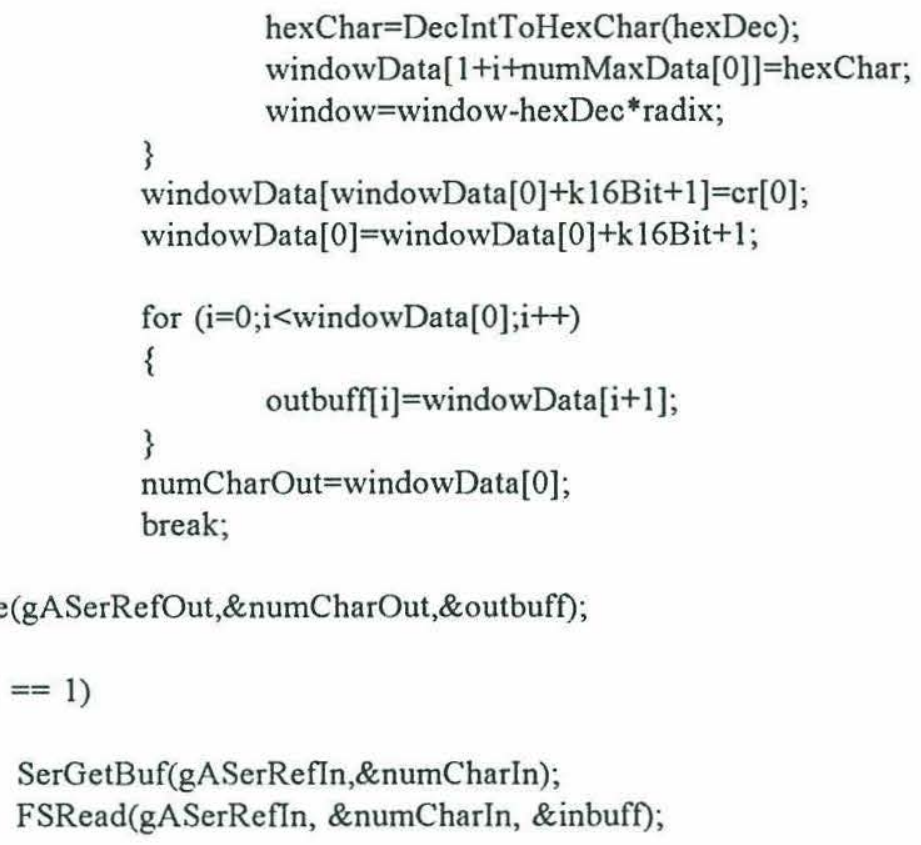



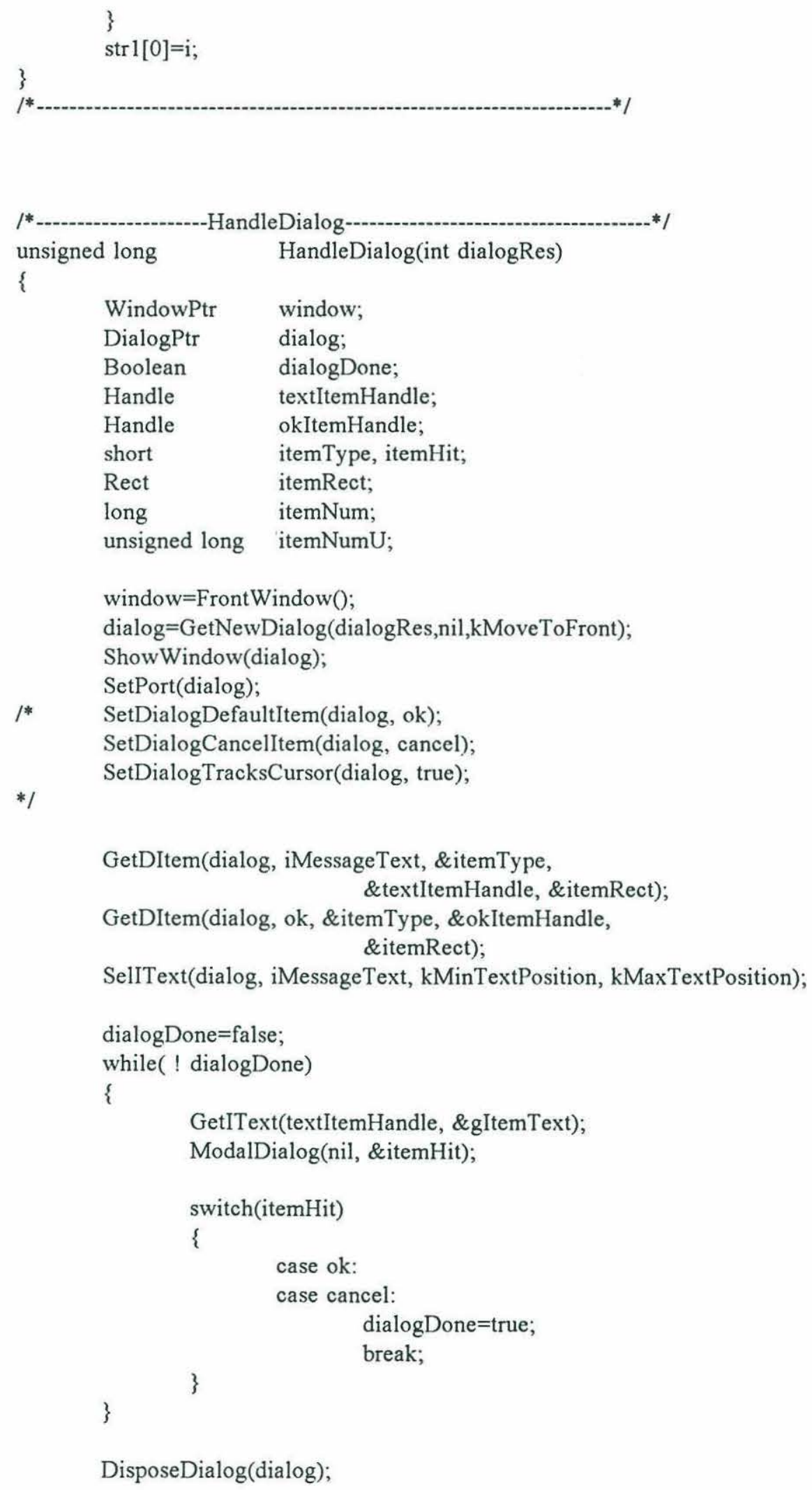
SelectWindow(window);

SetPort(window);

StringToNum(gItemText, \&itemNum);

itemNumU=itemNum;

return(itemNumU);

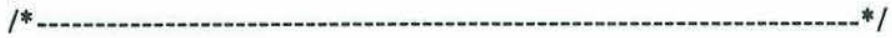

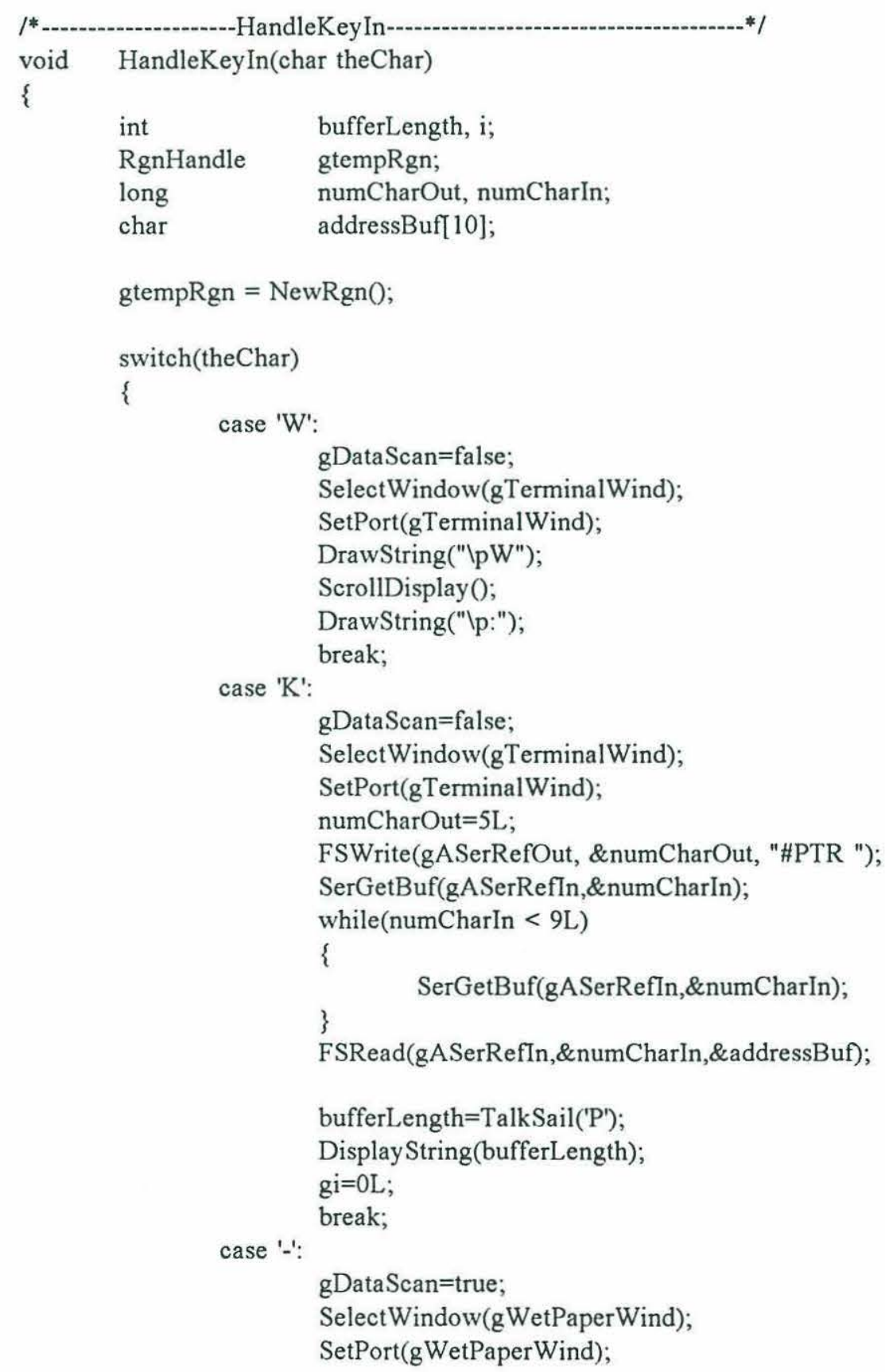




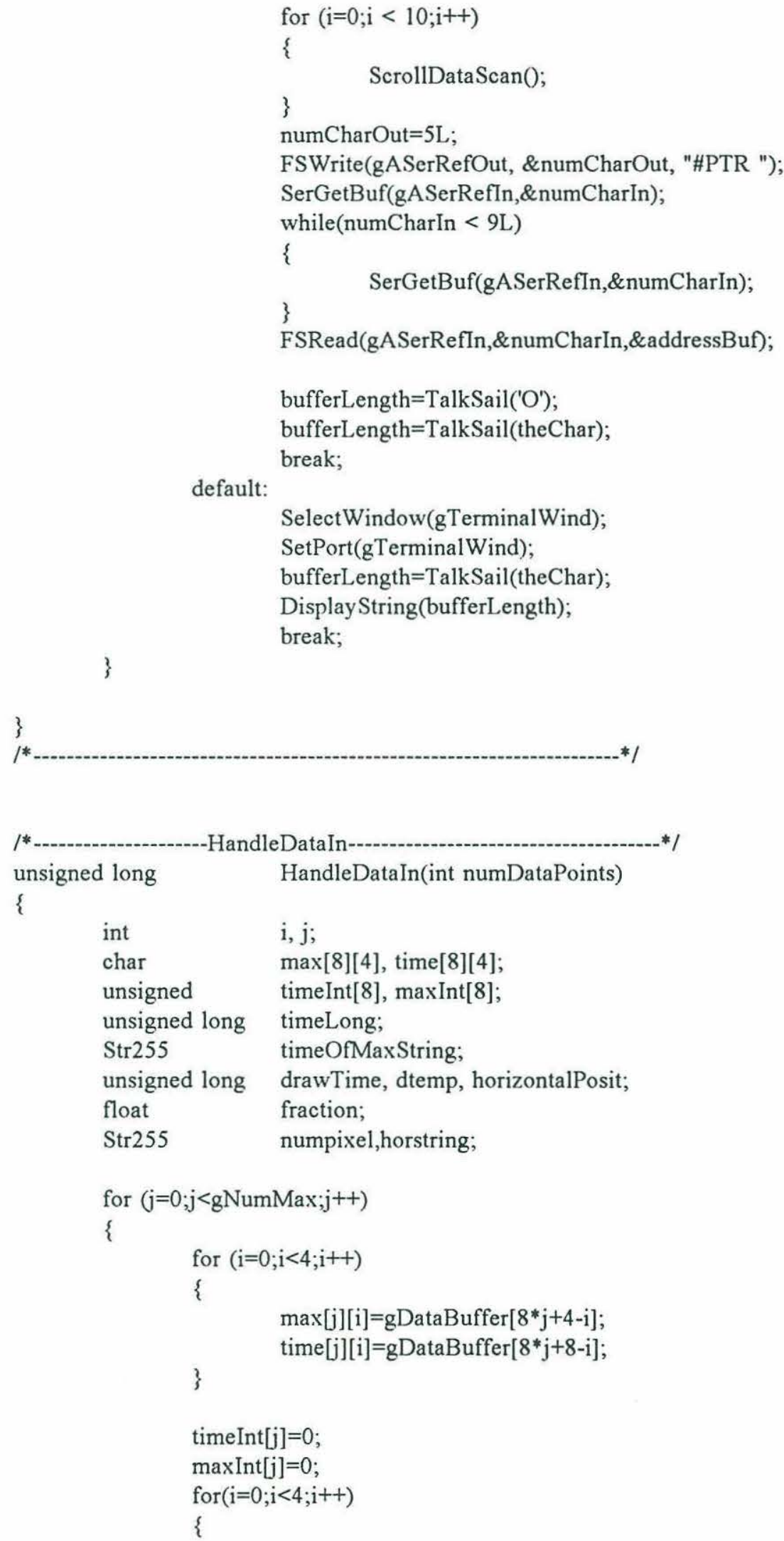




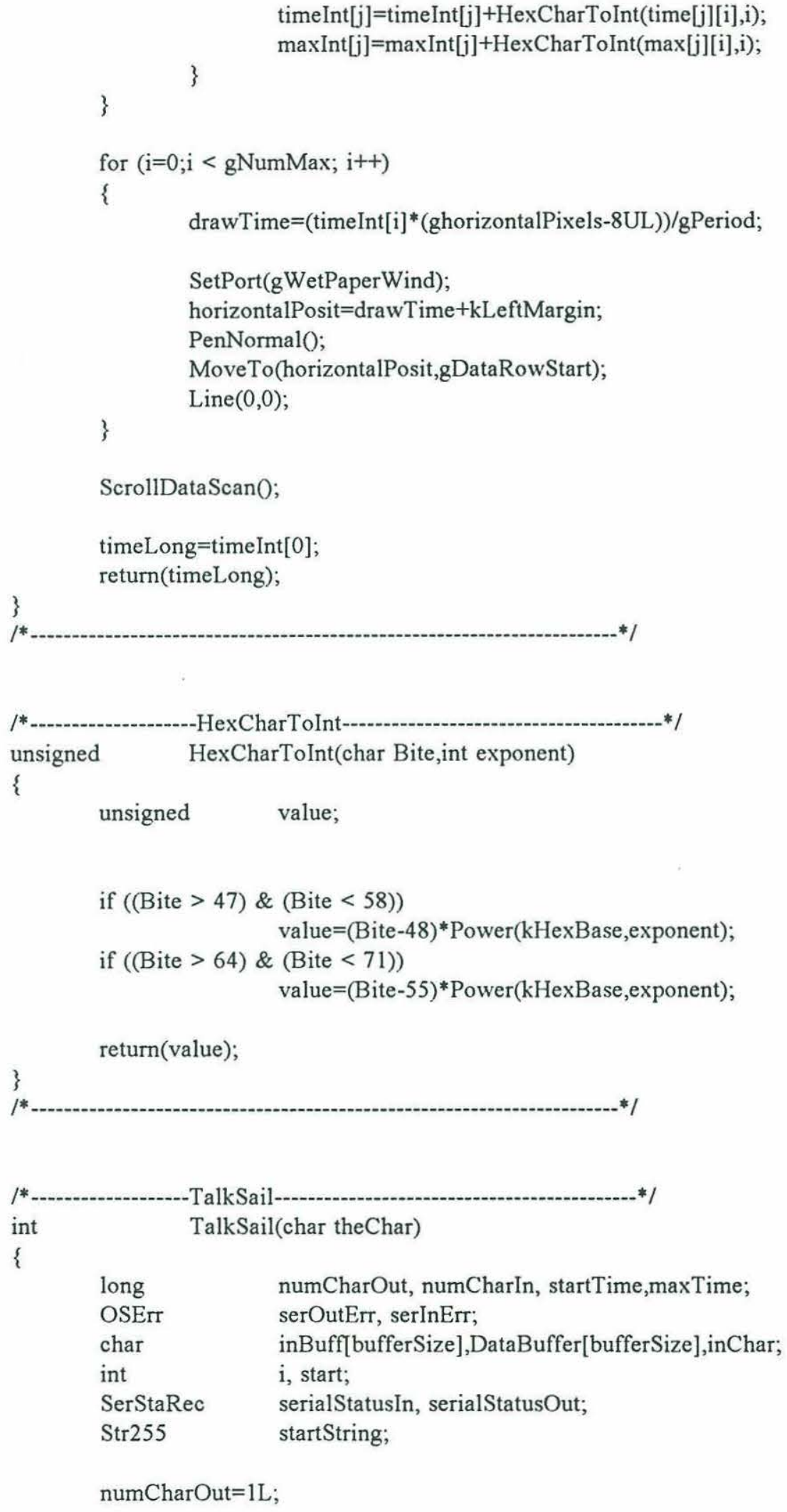


serOutErr=FSWrite(gASerRefOut, \&numCharOut, \&theChar); if (serOutErr ! = noErr)

\{

SysBeep(10);

DrawString("'pProblem sending the character out.");

ScrollDisplay();

\}

DrawString("\p:");

SerStatus(gASerRefOut,\&serialStatusOut);

while ((serialStatusOut.rdPend $!=0) \|($ serialStatusOut.wrPend $!=0)$ )

\{

\}

SerStatus(gASerRefOut,\&serialStatusOut);

SerStatus(gASerRefIn,\&serialStatusIn);

while(serialStatusIn.rdPend $!=0$ )

SerStatus(gASerRefIn,\&serialStatusIn);

start $=0$;

start Time $=$ TickCount () ;

$\max$ Time $=$ start Time $+20 \mathrm{~L}$;

while $(1==1)$

\{

SerGetBuf(gASerRefIn,\&numCharIn);

serInErr=FSRead(gASerRefIn, \&numCharIn, \&inBuff);

if (serInErr != noErr)

\{

SysBeep(10);

DrawString("\pProblem receiving characters.");

ScrollDisplay();

\}

DrawString("lp:");

memmove(\&gDataBuffer[start],\&inBuff,numCharIn);

start=start+LoWord(numCharIn);

if(inBuff[numCharIn-1L] == 03) break;

\}

if $($ (start $<2) \&($ TickCount ()$>\max$ Time $))$ break;

return(start);

\}

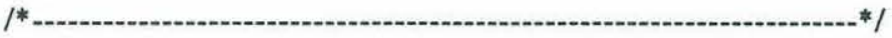

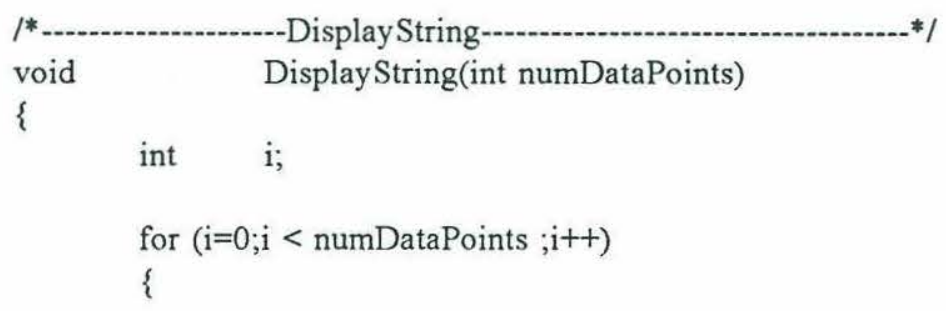




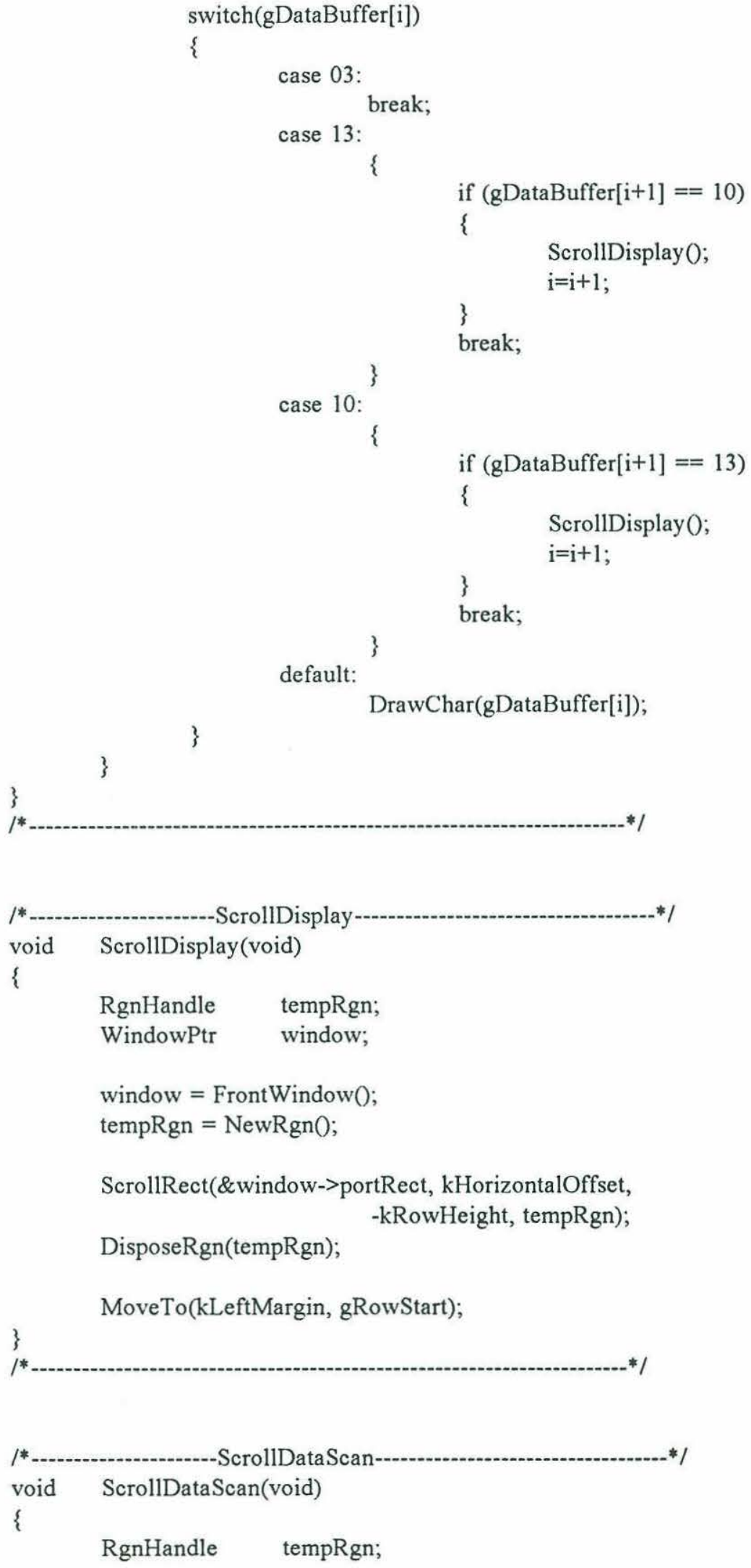




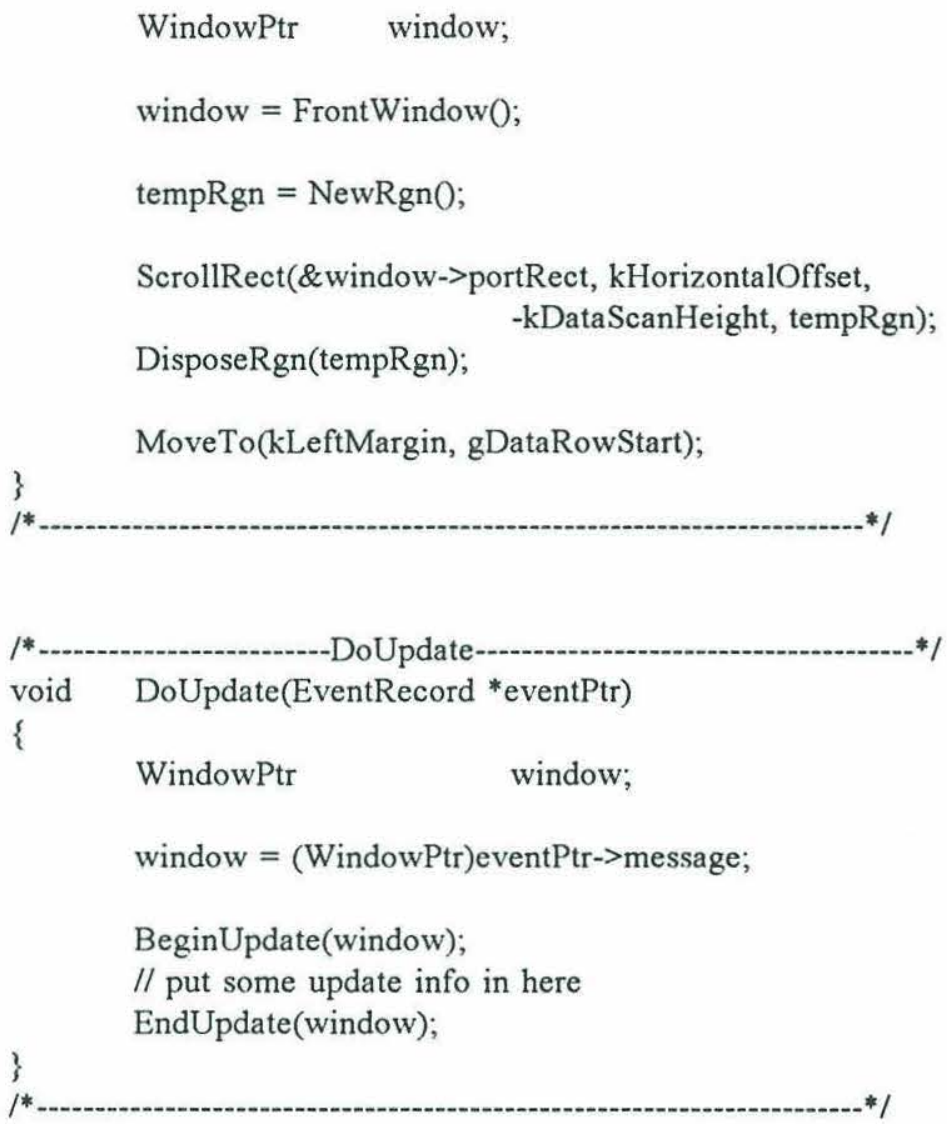




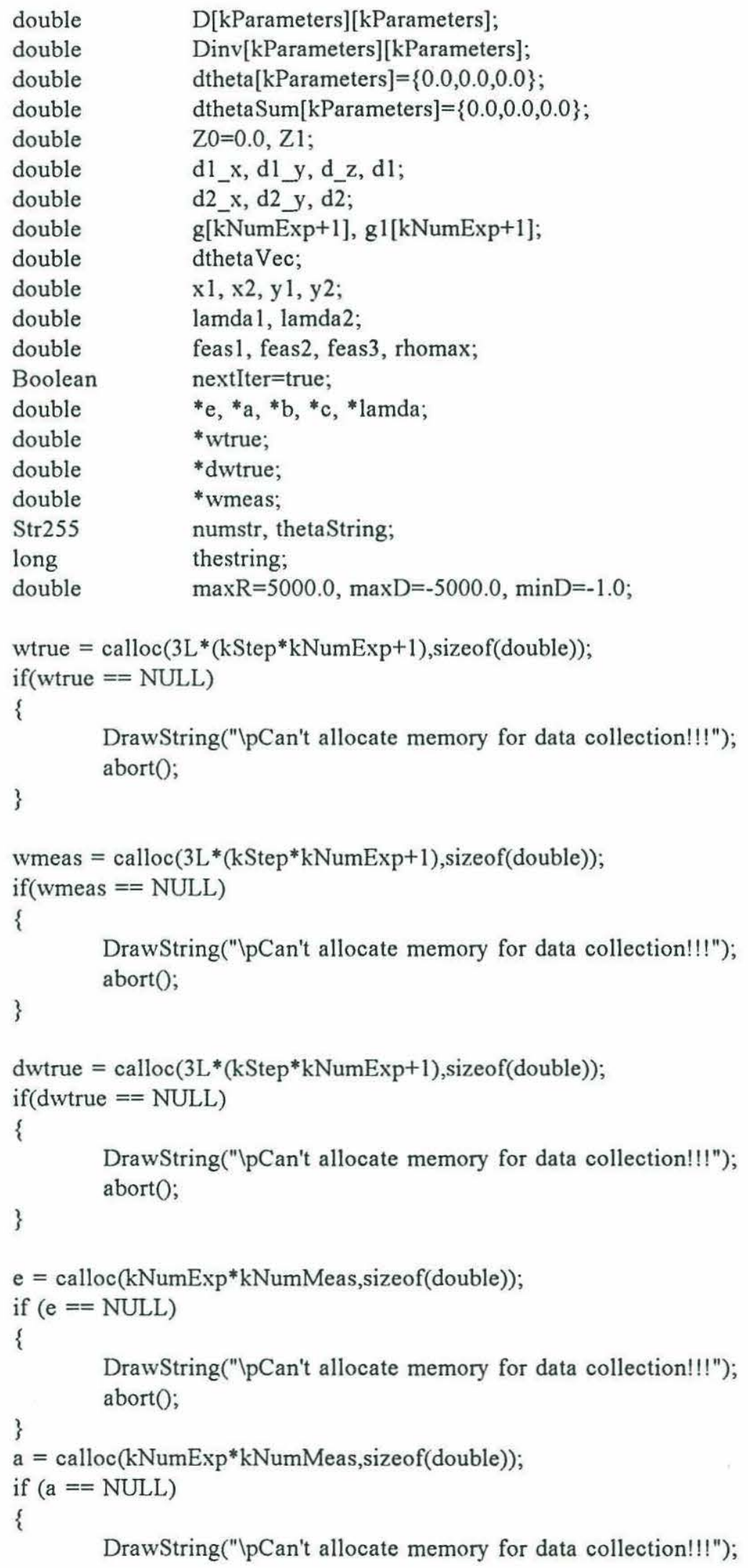




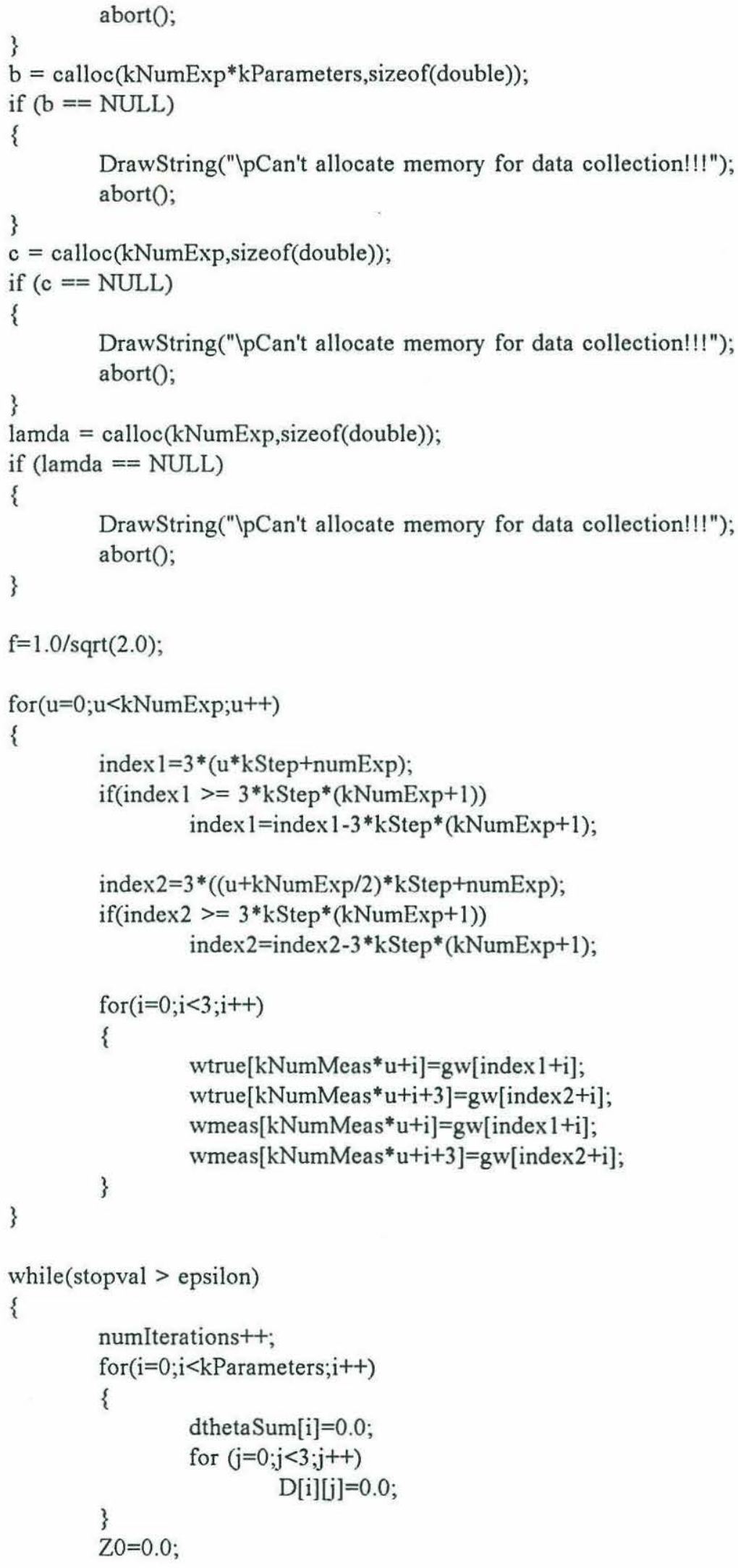




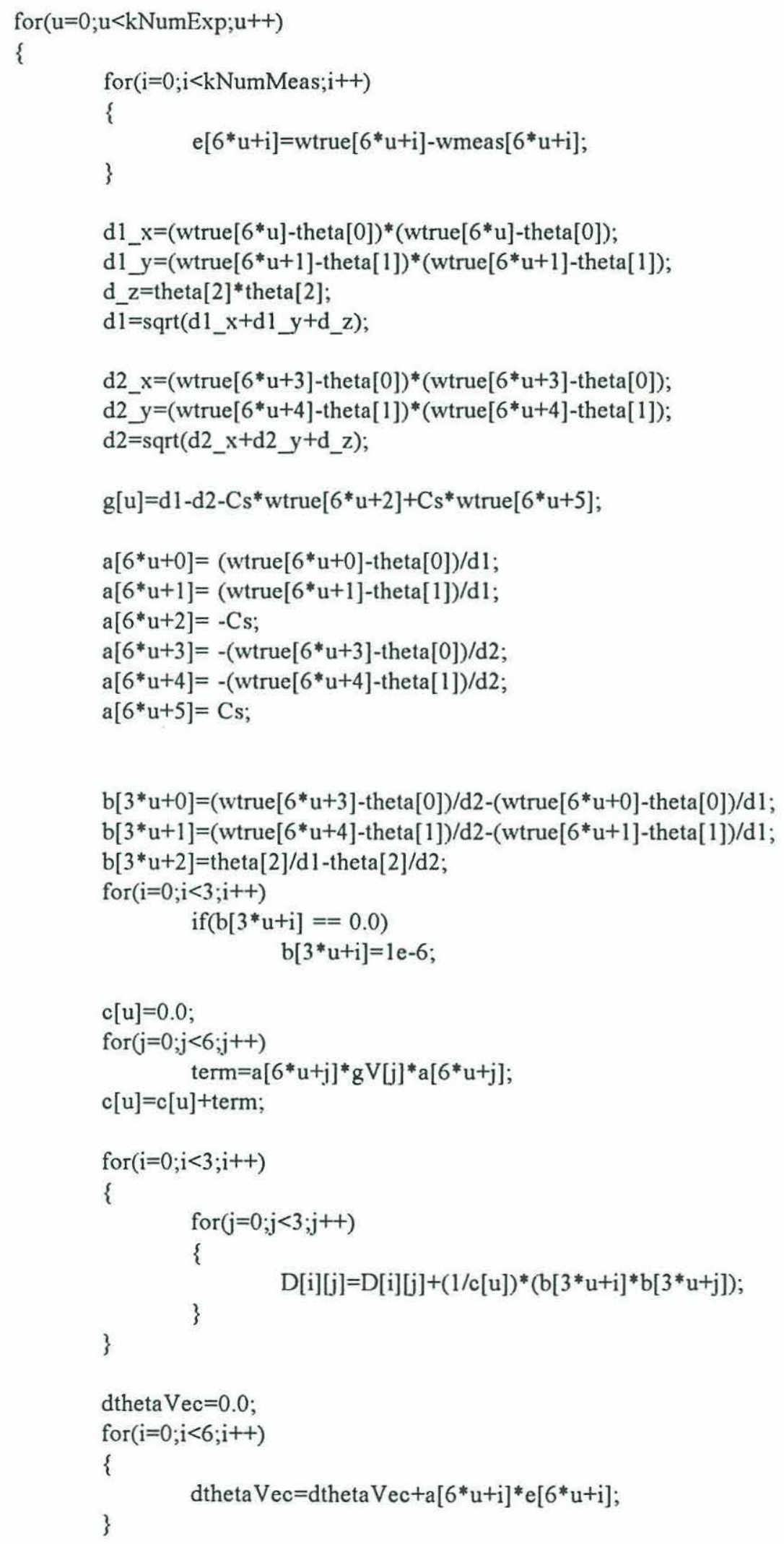




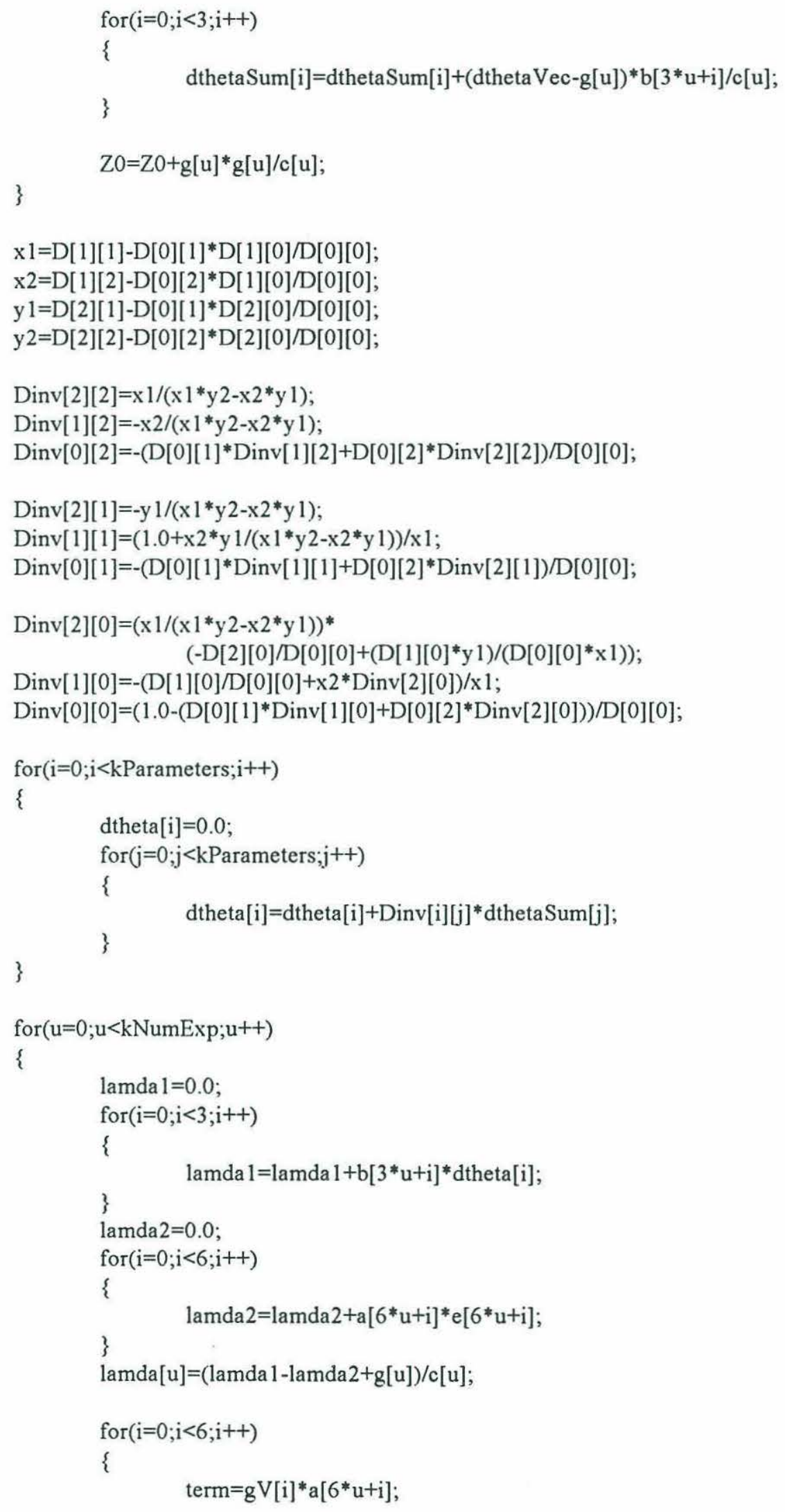




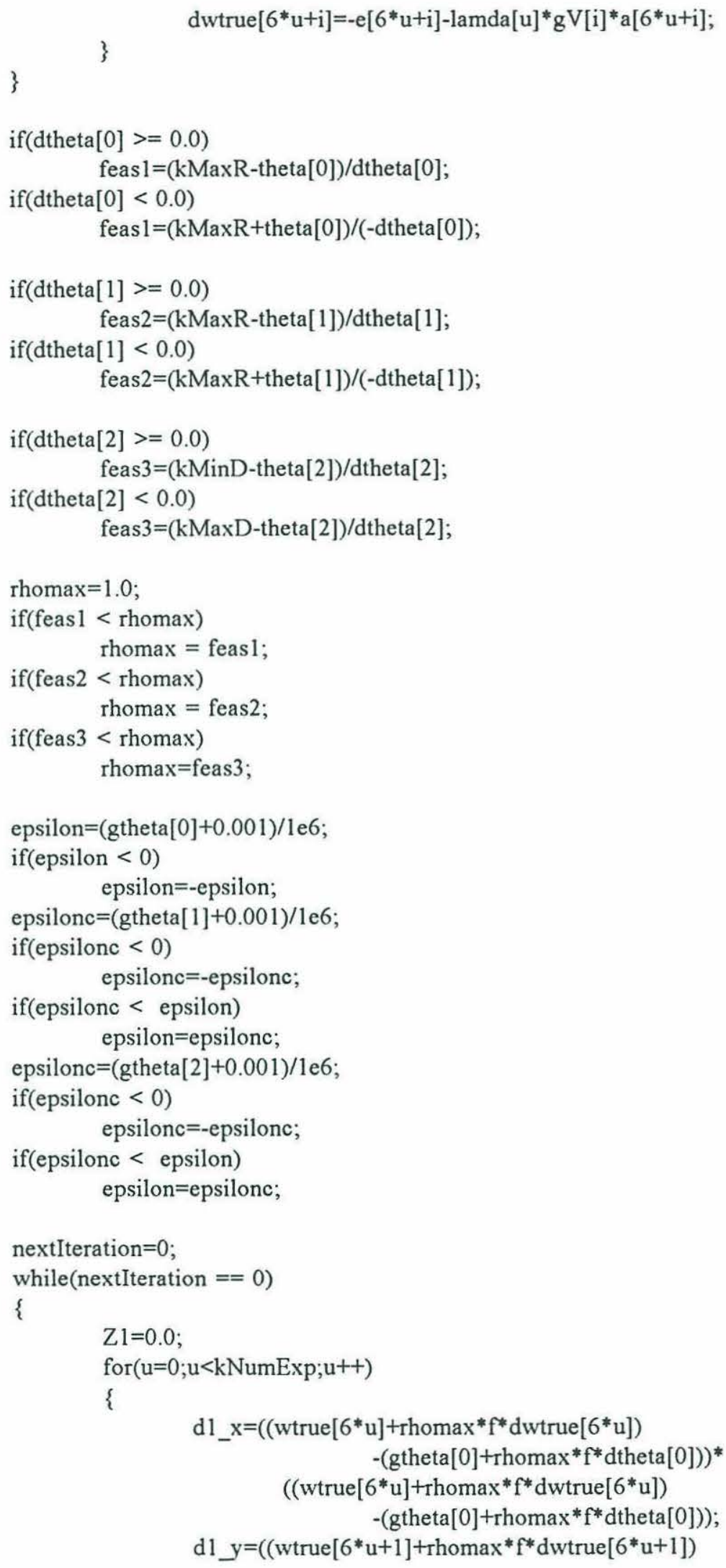




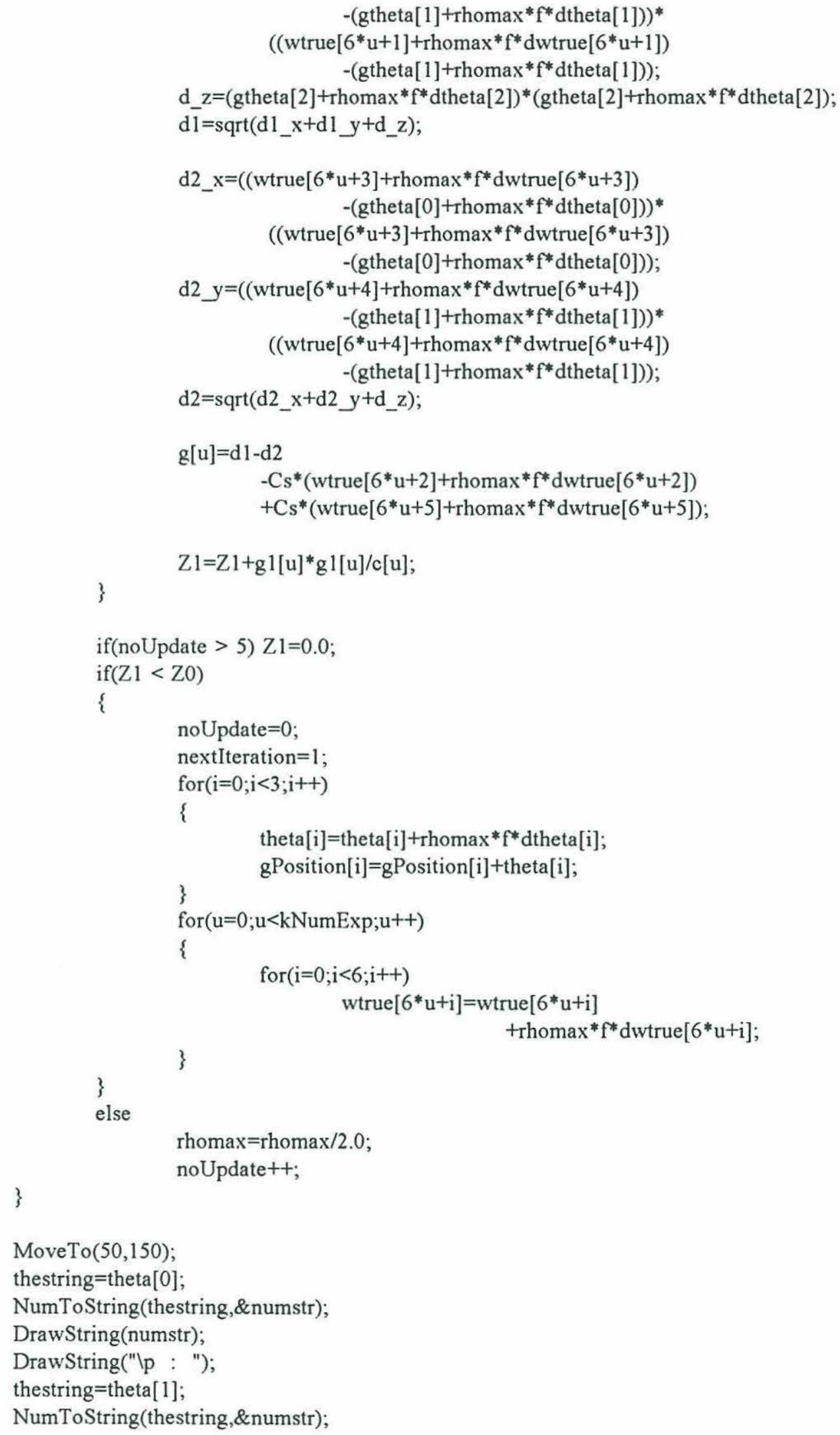




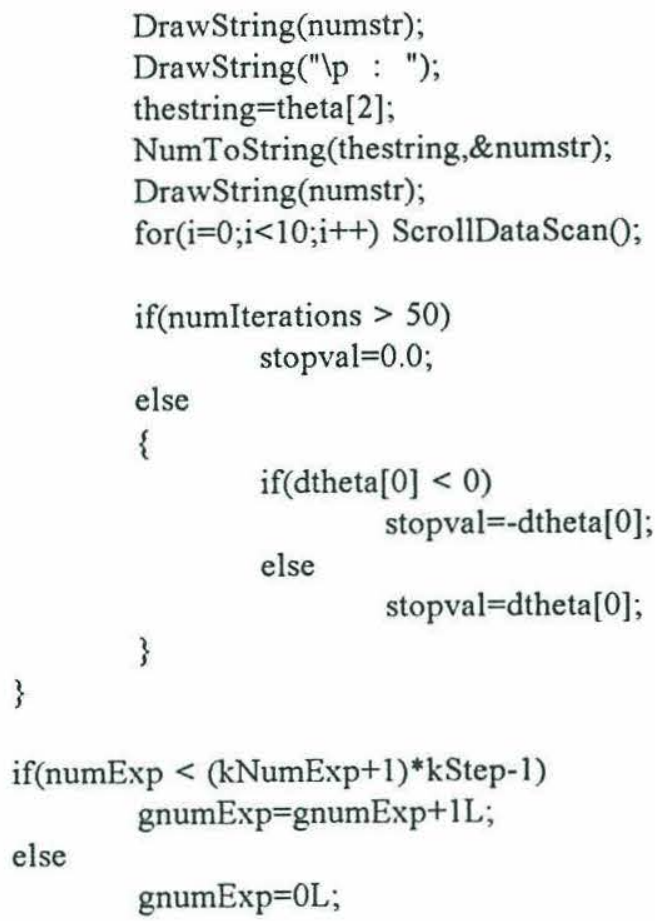




\section{References}

Allen, Mark R. and King, Louis A.. 1988. An adaptive Two Stage Kalman Structure for Passive Undersea Tracking. IEEE Transactions on Acoustics, Speech, and Signal Processing. Vol. 36, No 1, pages 3-9

Bard, Yonathan. 1974. Nonlinear Parameter Estimation. New York: Academic Press Inc.

Cheng, David K. 1983. Field and Wave Electromagnetic. Reading, Massachusetts: Addison-Wesley Publishing Company.

Friedlander, Benjamin. 1987. A Passive Localization Algorithm and Its Accuracy. IEEE Journal of Oceanic Engineering. Vol. OE-12, No. 1, pages 234-245

Horowitz and Hill. 1989. The Art of Electronics. New York: Cambridge University Press.

Millman, Jacob. 1979. Micro-Electronics: Digital and Analog Circuits and Systems. New York: McGraw-Hill Book Company.

Moose, Richard L.. 1987. Passive Range Estimation of an Underwater Maneuvering Target. IEEE Transactions on Acoustics, Speech, and Signal Processing. Vol. ASSP-35, No 3, pages 274-2859

Wenz, J.. 1962. Ambient Noise Spectra. Journal of the Acoustical Society of America. Vol. 34, p 1936.

Wilson, Oscar Bryan 1985. An Introduction To The Theory and Design of Sonar Transducers Monterey, California: Naval Postgraduate School. 